\title{
Integral estocástica e aplicações
}

\author{
Fabio Niski \\ DisSERTAÇÃO APRESENTADA \\ AO \\ Instituto De Matemática e Estatística \\ DA \\ Universidade DE SÃo PAUlO \\ PARA \\ OBTENÇÃO DO TÍTULO \\ $\mathrm{DE}$ \\ Mestre em CiênCias \\ Programa: Matemática Aplicada \\ Orientador: Prof. Dr. Edson de Faria
}

Durante o desenvolvimento deste trabalho o autor recebeu auxílio financeiro da CAPES

São Paulo, novembro de 2009 


\section{Integral estocástica e aplicações}

Este exemplar corresponde à redação final da dissertação devidamente corrigida e defendida por Fabio Niski

e aprovada pela Comissão Julgadora.

Banca Examinadora:

- Prof. Dr. Edson de Faria (orientador) - IME-USP.

- Prof. Dr. Henrique Von Dreifus - IME-USP.

- Prof. Dr. Nestor Felipe Caticha Alfonso - IF-USP. 


\section{Agradecimentos}

Agradeço aos professores que sempre muito solícitos e disponíveis, me ajudaram durante meus estudos no programa de mestrado: Adilson Simonis, Ângelo Barone, Manuel Garcia, Ricardo Freire, Sônia Garcia, Vladmir Belitsky e Walter Mascarenhas. Agradeço também a paciência do professor Daniel Tausk, por ter me ajudado com a prova de alguns resultados referentes a integração de funções de variação limitada e ao meu orientador Edson de Faria. Não posso deixar de citar a ajuda do professor Péter Medvegyev da universidade de Corvinus - Budapeste; desde o ínicio dos meus estudos sobre integração estocástica trocamos diversos e-mails que foram muito produtivos para minha formação acadêmica.

Aos colegas procastinadores do IME: Eduardo Oda, Getúlio Bulhões, Marcelo Caetano, Pavlos Konstadinidis, Pedro Peixoto e Ricardo Ribeiro muito obrigado por todas as agradáveis e divertidas perdas de tempo. Agradeço ao amigo Ilan Felts Almog por toda ajuda que precisei (e precisarei) com o idioma inglês e finalmente meu pai e minha mãe a quem eu dedico esse trabalho. 


\section{Resumo}

O aumento pelo interesse na teoria de integração estocástica é, basicamente, consequência da acirrada competição para entender, desenvolver e aplicar a matemática subjacente ao mercado mobiliário. Neste trabalho desenvolvemos, de maneira didática e visando aplicações, tal teoria.

Para tanto, começamos apresentando um desenvolvimento cuidadoso da teoria dos martingais e dos principais resultados de medida e probabilidade relacionados. Depois apresentamos de maneira formal a teoria de integração estocástica com respeito aos semi-martingais contínuos. Finalizamos com um tratamento das principais aplicações dessa teoria como a fórmula de Itô, uma introdução às equações diferenciais estocásticas e a fórmula de Feynman-Kac. Apresentamos também, em um apêndice, a teoria de mudança de medida e o teorema de Girsanov. Tentamos durante o trabalho apresentar exemplos relacionados com finanças e ilustrar a importância do movimento Browniano.

Palavras-chave: Integral Estocástica, Fórmula de Itô, Fórmula de Feynman-Kac, Finanças 


\section{Abstract}

The increasing interest in the theory of Stochastic Integration is due mainly to the competitive pressure to understand, develop and apply the underlying mathematics of security markets. In this work, we attempt to develop part of the theory in a didactical approach and focused toward some particular applications.

For this purpose, we begin by introducing a thorough development of Martingale theory and the main related results on Measure and Probability theory. We then present in a formal way the Stochastic Integration Theory with respect to continuous Semimartingales. Subsequentially, we show some of the theory's main applications, such as Itô's formula, an introduction to the theory of Stochastic Differential Equations and Feynman-Kac's formula. We also present in the appendix Girsanov's theorem and a construction of Brownian motion. During the development of this text we endeavored to enrich it by including examples relevant to finance and emphasizing the importance of the ubiquitous Brownian motion.

Keywords: Stochastic Integral, Itô's Formula, Feynman-Kac's Formula, Brownian motion, Finance 


\section{Sumário}

$\begin{array}{ll}\text { Índice de Notações } & 1\end{array}$

1 Introdução $\quad 3$

2 Conceitos básicos $\quad 5$

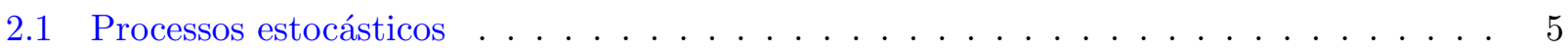

2.2 Filtrações e tempos de parada . . . . . . . . . . . . . . . . . . . . 7

2.3 Mensurabilidade progressiva . . . . . . . . . . . . . . . . . . . 13

2.4 Martingais . . . . . . . . . . . . . . . . . . . . 17

2.4.1 Martingais limitados em $\mathrm{L}^{2} \ldots \ldots \ldots \ldots \ldots \ldots \ldots$

2.5 Martingais locais . . . . . . . . . . . . . . . . . . 25

3 Variação quadrática $\quad 33$

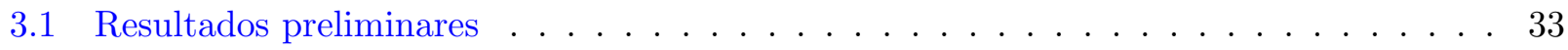

3.2 Variação quadrática para martingais contínuos uniformemente limitados . . . . . . . . 38

3.3 Variação quadrática para martingais locais . . . . . . . . . . . . . . . . . . . 45

3.4 Covariação quadrática . . . . . . . . . . . . . . . . . . . . . 50

3.5 Variação quadrática de martingais limitados $\mathrm{em}^{2} \ldots \ldots$. . . . . . . . . . . 53

3.6 Variação quadrática e semi-martingais . . . . . . . . . . . . . . . 56 
4 Integração estocástica $\quad 61$

4.1 Integração estocástica com respeito a processos de variação finita . . . . . . . . . . . . 61

4.2 A desigualdade de Kunita e Watanabe . . . . . . . . . . . . . . . . . . . . . 70

4.3 Integração estocástica com respeito a semi-martingais . . . . . . . . . . . . . . . . 74

4.4 O teorema da convergência dominada estocástica . . . . . . . . . . . . . . 92

4.5 Integração com respeito a semi-martingais vetoriais . . . . . . . . . . . . . . 105

4.6 A fórmula de Itô . . . . . . . . . . . . . . . . . . . . . . 106

4.6 .1 Notação diferencial . . . . . . . . . . . . . . . . . . . . . . . . . 113

4.6.2 Caracterização de Lévy e a tabela de cálculo para o movimento Browniano . 117

4.6.3 Funções harmônicas e o retorno do movimento Browniano para a origem . . . . 121

5 Conexões entre equações diferenciais estocásticas e parciais

5.1 Existência e unicidade para equações diferenciais estocásticas . . . . . . . . . . . . 125

5.2 O problema de Cauchy e a fórmula de Feynman-Kac . . . . . . . . . . . . . . 138

$\begin{array}{ll}\text { A Teorema da classe monótona } & 151\end{array}$

B Convergência de variavéis aleatórias $\quad 153$

$\begin{array}{lr}\text { C Esperança condicional } & 157\end{array}$

$\begin{array}{ll}\text { D Uma construção do movimento Browniano } & 165\end{array}$

D.1 Introdução . . . . . . . . . . . . . . . . . . . . . . . . 165

D.2 Fatos sobre a distribuição Normal e processos Gaussianos . . . . . . . . . . . . . 166

D.3 Conceitos de Análise Funcional . . . . . . . . . . . . . . . . . . 170

D.4 A construção do movimento Browniano em $[0,1] \ldots \ldots$. . . . . . . . . . . 171

D.4.1 As três primeiras propriedades . . . . . . . . . . . 171 
D.4.2 A última propriedade . . . . . . . . . . . . . . . . 175

D.5 Extensão para $[0, \infty) \ldots \ldots \ldots \ldots \ldots \ldots$

$\begin{array}{ll}\text { E Teorema de Girsanov } & 181\end{array}$

E.1 Teorema de Bayes . . . . . . . . . . . . . . . . . . . . . . 181

E.2 Mudança de medida . . . . . . . . . . . . . . . . . . . . . . . . . 184

E.2.1 A fórmula de Girsanov . . . . . . . . . . . . . . . . . . . . . . . 184

E.2.2 A exponencial de Doléans . . . . . . . . . . . . . . . . . 186

E.3 Teorema de Girsanov . . . . . . . . . . . . . . . . . . . . . . . 188

E.3.1 A condição de Novikov . . . . . . . . . . . . . . . . . . . . . . . 193 


\section{Índice de Notações}

$\alpha_{\mathbf{M}} \quad$ Medida de Dóleans, página 75

$\mathscr{F}_{\infty} \quad \sigma$-álgebra no infinito, página 7

$\mathscr{F}_{t}^{\mathbf{X}} \quad \sigma$-álgebra gerada pelos valores do processo até o instante $t$, página 7

$\Lambda^{2}(\mathbf{M})$, página 89

$\Lambda_{b} \quad$, página 92

$\mathcal{E}(\mathbf{L}) \quad$ Exponencial de Doléans, página 186

$\mathcal{H}^{2} \quad$ Espaço dos martingais contínuos à direita limitados em $\mathrm{L}^{2}$, página 23

$\mathcal{L}(\mathbf{X})$, página 90

$\mathcal{L}^{2}(\mathbf{M})$, página 75

$\mathcal{L}^{1}(\mathbf{A})$, página 65

$\mathcal{L}_{\text {loc }}^{1}(\mathbf{A})$, página 64

$\mathcal{L}_{\text {loc }}^{2}(\mathbf{M})$, página 85

$\mathcal{S}^{d} \quad$, página 105

$\mathfrak{P}_{g} \quad \sigma$-álgebra dos conjuntos progressivamente mensuráveis, página 13

$\|\cdot\|_{\mathbf{M}} \quad$ Norma no espaço $\mathcal{L}^{2}(\mathbf{M})$, página 75

$\|M\|_{\mathcal{H}^{2}}$ Norma no espaço dos martingais limitados em $\mathrm{L}^{2}$, página 23 
$\mathrm{cH}^{2} \quad$ Espaço dos martingais contínuos e limitados em $\mathrm{L}^{2}$, página 24

c $\mathcal{H}_{0}^{2} \quad$ Espaço dos martingais contínuos, limitados em $\mathrm{L}^{2}$ e que valem 0 em $t=0$, página 24

$\llbracket \mathbf{M}, \mathbf{N} \rrbracket$ Covariação quadrática entre os processos $\mathbf{M}$ e $\mathbf{N}$, página 50

$\llbracket \mathbf{M} \rrbracket \quad$ Variação quadrática do processo estocástico $\mathbf{M}$, página 38

$u_{\mathbf{X}} \quad$ Processo compensador do semi-martingal $\mathbf{X}$, página 56 


\section{Capítulo 1}

\section{Introdução}

I turn away in fright and horror from this lamentable plague of functions that do not have derivatives.

Charles Hermite

Por ser um componente matemático fundamental para a teoria de apreçamento de derivativos financeiros, a teoria de integração estocástica goza de fama e popularidade nos dias de hoje. Existem muitos livros que introduzem a teoria e só tratam do caso quando o integrador é um movimento Browniano. Tal escolha se justifica por motivos de espaço e redução dos pré-requisitos matemáticos visto que muitos leitores destes textos estão interessados apenas em algumas aplicações elementares para finanças e não tem tempo e/ou base matemática para a estudar teoria mais geral. Neste trabalho, no entanto, seguiremos a filosofia de [29], [15] e [23]. Isso significa que pelo menos do ponto de vista pedagógico, acreditamos que para um primeiro estudo da teoria, a abordagem mais simples e bem resolvida para a integral estocástica é quando consideramos os semi-martingais contínuos como integradores. Essa abordagem é suficientemente abstrata para cobrir os aspectos mais importantes da teoria. Por outro lado, quando se considera apenas o movimento Browniano como integrador, a verdadeira importância do conceito de variação quadrática é omitida. Uma generalização do conceito de integral estocástica que apresentaremos neste trabalho é necessária quando queremos considerar semi-martingais não contínuos como integradores (processos de Poisson, por exemplo). Para tratar disso é necessário introduzir o conceito de predictable processes e a teoria se torna consideravalmente mais complicada. Para tal grau de generalidade, indicamos o leitor para o livro de Métivier [17] ou também o livro de Protter [21]. 
O objetivo principal deste trabalho é definir e apresentar as principais propriedades da integral estocástica $\int \mathbf{H} d \mathbf{X}$ onde $\mathbf{X}$ é um semi-martingal contínuo e $\mathbf{H}$ está em uma classe conveniente de integrantes. Para isso, no capítulo 2 desenvolvemos a teoria de martingais em tempo contínuo seguindo [15], [29] e [16]. No capítulo 3 estudamos o conceito de variação quadrática, que é pedra fundamental para a definição de integral estocástica. Nesse capítulo seguimos [29]. O capítulo 4 é o ponto central do trabalho e está baseado em [29] e [16]. Lá desenvolvemos a teoria de integração estocástica culminando na tão falada fórmula de Itô. Neste trabalho provamos a fórmula de Itô para funções definidas em um aberto. Essa versão é a mais usada em aplicações e em muitos textos ela é (curiosamente) provada apenas para funções definidas em $\mathbb{R}^{n}$ inteiro. Como aplicação da fórmula de Itô, mostramos a primeira conexão entre processos estocásticos e equações diferenciais parciais: Através da fórmula de Dynkin, provaremos propriedades do retorno para a origem do movimento Browniano. No capítulo 5 estudamos equações diferencais estocásticas e conexões com equações diferenciais parciais parabólicas. Neste capítulo, seguindo [23], deixamos um pouco de lado a generalidade e nos focamos no caso Browniano pois a grande maioria das aplicações surgem nesse paradigma. Mais especificamente, nesta última parte do trabalho, definimos o que se entende por equação diferencial estocástica e provamos resultados de existência e unicidade. Finalizamos apresentando uma interessante relação entre a solução de equações diferenciais estocásticas e certos tipos de equações diferenciais parciais expressa pela fórmula de Feynman-Kac. Este trabalho não foi focado para as aplicações em finanças mas nos exemplos, sempre que possível, trabalhamos pontos que são relevantes para o mercado financeiro. Um pouco da história da teoria está dispersa pelo texto. Para o leitor mais interessado nos detalhes do desenvolvimento histórico da teoria da integração estocástica recomendamos o artigo [22].

A escolha dos tópicos dos apêndices foi feita com dois objetivos. O primeiro foi organizar de maneira rápida e sucinta resultados técnicos que são necessários ao longo do texto. É o caso dos dois primeiros apêndices. O terceiro apêndice também tem esse espirito, mas dada a relevância do conceito de esperança condicional fomos mais a fundo e disponibilizamos um tratamento mais completo do tópico. O segundo objetivo foi apresentar algumas idéias que, apesar de não serem utilizadas diretamente no trabalho, são interessantes por sí só. Do ponto de vista histórico e de fundamentos, escolhemos incluir um apêndice que trata da construção do movimento Browniano seguindo as idéias de Lévy e Ciesielski. Do ponto de vista de aplicações, o nosso último apêndice versa sobre o teorema de Girsanov, uma ferramenta muito utilizada em finanças para modelagem e apreçamento de derivativos. 


\section{Capítulo 2}

\section{Conceitos básicos}

Structures are the weapons of the mathematician.

Nicolas Bourbaki

Neste capítulo, faremos uma revisão dos conceitos fundamentais para os resultados que aparecerão depois neste trabalho.

\subsection{Processos estocásticos}

Ao longo de todo o texto, denotaremos a $\sigma$-álgebra de Borel em $\mathbb{R}$ por $\mathscr{B}_{\mathbb{R}}$. Denotaremos também $\mathbb{R}_{+} \doteq[0, \infty)$.

Definição 2.1. Sejam $\Theta$ um conjunto de índices e $d \in \mathbb{N}$. Suponha que para cada $t \in \Theta$, $X_{t}$ é uma variável aleatória definida no espaço de probabilidade $(\Omega, \mathscr{F}, \mathrm{P})$, isto é, uma aplicação $\mathscr{F} / \mathscr{B}_{\mathbb{R}^{d}}$-mensurável. Nessas condições, dizemos que a família $\left\{X_{t}: t \in \Theta\right\}$ é um processo estocástico. Usaremos a notação em negrito $\mathbf{X}$ para denotar um processo estocástico.

Para cada $\omega \in \Omega$, dizemos que a função $t \mapsto X_{t}(\omega) \doteq X(t, \omega)$ é uma trajétoria do processo $\mathbf{X}$. Diremos também que um processo $\mathbf{X}$ é q. s. contínuo quando para quase todo $\omega \in \Omega$, as trajetórias de $\mathbf{X}$ forem funções contínuas.

Se $X$ é uma aplicação $\mathscr{F} / \mathscr{B}_{\mathbb{R}^{d}}$-mensurável é comum escrever apenas que $X$ é $\mathscr{F}$-mensurável e também utilizamos a notação $X \in \mathscr{F}$ quando estiver claro que $X$ é uma variavel aleatória e não um conjunto. 
Definição 2.2. Dizemos que dois processos estocásticos $\mathbf{X}$ e $\mathbf{Y}$ são modificações um do outro quando para cada $t \in \Theta$,

$$
X_{t}=Y_{t} \text { q. s. }
$$

Dizemos que $\mathbf{X}$ e $\mathbf{Y}$ são indistinguíveis quando para quase todo $\omega \in \Omega$,

$$
X_{t}(\omega)=Y_{t}(\omega) \quad \forall t \in \Theta
$$

Quando $\Theta$ for enumerável, os dois conceitos da definição anterior coincidem. Quando $\Theta=\mathbb{R}_{+}$, é evidente que a indistinguibilidade entre $\mathbf{X}$ e $\mathbf{Y}$ implica que um é uma modificação do outro. Apresentamos uma recíproca parcial deste resultado.

Proposição 2.3. Sejam $\mathbf{X}$ e $\mathbf{Y}$ dois processos estocásticos q. s. contínuos à direita ou à esquerda $e$ $\Theta=\mathbb{R}_{+}$. Nessas condições se um é uma modificação do outro então os processos são indistinguíveis.

Demonstração. Sejam X e $\mathbf{Y}$ dois processos q. s. contínuos à direita, um sendo modificação do outro. Sejam também $\Lambda_{\mathbf{X}}$ e $\Lambda_{\mathbf{Y}}$ conjuntos de medida nula em $(\Omega, \mathscr{F}, \mathrm{P})$ tais que $X(\cdot, \omega)$ é contínuo à direita em $\Lambda_{\mathbf{X}}^{c}$ e $Y(\cdot, \omega)$ é contínuo à direita em $\Lambda_{\mathbf{Y}}^{c}$. Considere $\left\{r_{n}\right\}$ uma enumeração dos racionais em $\mathbb{R}_{+}$ com $r_{0}=0$. Temos então que para cada $n \in \mathbb{N}$ existe um conjunto de medida nula $\Lambda_{n}$ em $(\Omega, \mathscr{F}, \mathrm{P})$ tal que para $\omega \in \Lambda_{n}^{c}$ temos que $X\left(r_{n}, \omega\right)=Y\left(r_{n}, \omega\right)$. Desta maneira, defina $\Lambda \doteq \Lambda_{\mathbf{X}} \cup \Lambda_{\mathbf{Y}} \cup\left(\cup_{n \in \mathbb{N}} \Lambda_{n}\right)$. Temos que $\Lambda$ é um conjunto de medida nula e para $\omega \in \Lambda^{c}$,

$$
X\left(r_{n}, \omega\right)=Y\left(r_{n}, \omega\right) \quad \forall n \in \mathbb{N} .
$$

Agora seja $t \in(0, \infty)$ e seja $\left\{s_{k}\right\}$ uma sequência em $\mathbb{Q}$ tal que $s_{k} \downarrow t$. Então, quando $\omega \in \Lambda^{c}$, temos que $X\left(s_{k}, \omega\right)=Y\left(s_{k}, \omega\right)$ para todo $k \in \mathbb{N}$. Usando a contínuidade à direita das trajétorias, segue que portanto que para $\omega \in \Lambda^{c}$, vale que $X(t, \omega)=Y(t, \omega)$. Para o caso $t=0$, note que como $\Lambda^{c} \subset \Lambda_{0}^{c}$ também vale que para $\omega \in \Lambda^{c}, X(0, \omega)=Y(0, \omega)$. Portanto, foi provada a existência de um conjunto $\Lambda$ de medida nula em $(\Omega, \mathscr{F}, \mathrm{P})$ tal que quando $\omega \in \Lambda^{c}, X(t, \omega)=Y(t, \omega)$ para todo $t \in \mathbb{R}_{+}$.

Para o caso de processos contínuos pela esquerda, basta tomar a sequência aproximante $\left\{s_{k}\right\}$ racional de $t$ de tal forma que $s_{k} \uparrow t$ e a demonstração é análoga.

Um dos principais exemplos de processo estocástico é movimento Browniano, cuja definição veremos abaixo e a existência nós apresentamos no apêndice D. 


\section{$2.2 \quad$ Filtrações e tempos de parada}

Avisamos o leitor que o conjunto de índices $\Theta$ será, daqui para frente, $\mathbb{R}_{+}$ou $\mathbb{N}$ ou subconjuntos destes conjuntos.

Para modelar conceitos dinâmicos e que mudam ao longo do o tempo, é natural incluir em nosso espaço de probabilidade um conceito de fluxo de informações que aumentam (ou pelo menos, não diminuem) com o passar do tempo. Este é o propósito da próxima

Definição 2.4. Uma filtração em um espaço mensurável $(\Omega, \mathscr{F})$ é uma família de sub- $\sigma$-álgebras crescentes de $\mathscr{F}$. Denotamos esta família por $\left\{\mathscr{F}_{t}\right\}_{t \in \Theta}$ ou simplesmente $\left\{\mathscr{F}_{t}\right\}$. A condição de ser crescente significa precisamente que $\mathscr{F}_{s} \subseteq \mathscr{F}_{t}$ para $s<t$. Quando $\mathscr{F}_{t}=\bigcap_{s>t} \mathscr{F}_{s}$ para todo $t$, dizemos que a filtração $\left\{\mathscr{F}_{t}\right\}$ é contínua à direita.

De acordo com essa definição e no contexto de modelagem em finanças, podemos pensar que $\mathscr{F}_{t}$ é a informação sobre o mercado que está disponível para um observador até o instante $t$.

Definição 2.5. Dizemos que um processo estocástico em $(\Omega, \mathscr{F})$ é adaptado à filtração $\left\{\mathscr{F}_{t}\right\}$ quando $X_{t}$ for $\mathscr{F}_{t}$-mensurável para cada t. Associada a uma filtração $\left\{\mathscr{F}_{t}\right\}_{t \geq 0}$, definimos a $\sigma$-álgebra no infinito, $\mathscr{F}_{\infty} \doteq \sigma\left(\bigcup_{t \geq 0} \mathscr{F}_{t}\right)$.

Sendo $\mathbf{X}$ um processo estocástico que representa o preço de um certo ativo financeiro, podemos expressar o conceito de adaptabilidade do processo $\mathbf{X}$ com respeito à filtração dizendo que o preço em um certo momento $t$ é formado com base nas informações disponíveis em $\mathscr{F} t$.

Seja X um processo estocástico. Denotamos por $\mathscr{F}_{t}^{\mathbf{X}}$ a $\sigma$-álgebra gerada pelos valores do processo até o instante $t$, em símbolos:

$$
\mathscr{F}_{t}^{\mathbf{X}}=\sigma\left(\left\{X_{s}: s \in \Theta, s \leq t\right\}\right) .
$$

Chamamos a filtração $\left\{\mathscr{F}_{t}^{\mathbf{X}}\right\}_{t}$ de filtração gerada pelo processo $\mathbf{X}$. É claro pela definição que todo processo estocástico é adaptado à filtração gerada por ele próprio. Dizemos também que $\left\{\mathscr{F}_{t} \mathbf{X}\right\}_{t}$ é a filtração natural associada ao processo $\mathbf{X}$.

O exemplo mais importante de processo adaptado nesse trabalho é apresentado na próxima

Definição 2.6. Dizemos que um processo estocástico B é um movimento Browniano padrão com respeito à filtração $\left\{\mathscr{F}_{t}\right\}_{t \in \mathbb{R}_{+}}$quando satisfizer as seguintes propriedades 
i) $B_{0}=0$, P-q.s..

ii) $B_{t}-B_{s}$ é independente de $\mathscr{F}_{s}$ para $0 \leq s \leq t$.

iii) $B_{t}-B_{s} \sim N(0, t-s)$ para $0 \leq s \leq t$.

Observamos que ao nos referirmos a um movimento Browniano padrão estamos assumindo implicitamente uma filtração. No apêndice D provamos a existência de um movimento Browniano mas sem fazer referência a qualquer filtração. No entanto, prova-se* que a existência do movimento Browniano implica na existência de um movimento Browniano padrão. Para isto, basta considerar a $\sigma$-álgebra natural aumentada $\overline{\mathscr{F}}_{t}^{\mathbf{B}}$ de $B_{t}$ que é definida como $\overline{\mathscr{F}}_{t}^{\mathbf{B}} \doteq \sigma\left(\mathscr{F}_{t}^{\mathbf{B}} \cup \mathfrak{N}\right)$ onde $\mathfrak{N}$ denota a coleção de todos os conjuntos de medida nula no espaço de probabilidade completo $(\Omega, \mathscr{F}, \mathrm{P})$. Dessa forma, B é um movimento Browniano padrão com respeito à $\left\{\overline{\mathscr{F}}_{t}^{\mathbf{B}}\right\}_{t}$.

Vamos agora introduzir um importante o conceito.

Definição 2.7. Dizemos que uma função $\tau: \Omega \rightarrow \overline{\mathbb{R}}_{+}$é um tempo de parada relativo à filtração $\left\{\mathscr{F}_{t}\right\}_{t \geq 0}$ quando para cada $t \geq 0$ valer que

$$
\{\omega \in \Omega: \tau(\omega) \leq t\} \in \mathscr{F}_{t}
$$

Note que como $\mathscr{F}_{\infty}=\sigma\left(\cup_{t \in \mathbb{R}_{+}} \mathscr{F}_{t}\right)$, um tempo de parada $\tau$ é $\mathscr{F}_{\infty}$-mensurável e em particular, $\{\omega \in \Omega: \tau(\omega)=\infty\} \in \mathscr{F}_{\infty}$.

Exemplo 2.8 (Estratégias para um apostador). Critérios de parada para um jogador sem informações futuras ilustram o conceito de tempo de parada. Para isso, sejam $\Theta=\mathbb{N}$ e $\Omega$ o espaço de todas as sequências infinitas onde cada elemento de cada sequência toma apenas os valores 1 ou -1 . Assim, $\omega \in \Omega$ significa que $\omega: \mathbb{N} \rightarrow\{-1,1\}$. Defina $\mathscr{F}_{n}$ como a menor $\sigma$-álgebra que contém todos os conjuntos da forma $\left\{\omega \in \Omega: \omega(1)=\alpha_{1}, \ldots, \omega(n)=\alpha_{n}\right\}$, onde $\alpha_{1}, \ldots, \alpha_{n} \in\{-1,1\}$. Seja $\mathscr{F}$ a menor $\sigma$-álgebra que contém todas $\mathscr{F}_{n}, n \geq 1$. Construído dessa forma, o espaço $(\Omega, \mathscr{F})$ é um modelo de uma sequência infinita de apostas de tal forma que o resultado de cada aposta é o ganho ou a perda de uma unidade monetária. Um exemplo de tempo de parada nesse contexto é o primeiro momento quando o jogador acumula, digamos, cinco unidades monetárias, isto é

$$
\tau(\omega) \doteq \min _{n}\left\{\sum_{i=1}^{n} \omega(i)=5\right\} .
$$

\footnotetext{
* Veja [28] - remark 13.18, página 273 para um tratamento detalhado sobre isso.
} 
A definição de tempo de parada não permite critérios de parada dependam de informações privilegiadas (futuras), por exemplo,

$$
\sigma(\omega) \doteq \min _{n}\{\omega(n+1)=-1\}
$$

(parar o jogo antes da primeira vez que ocorre uma perda) seria uma estratégia muito confortável para o apostador. É fácil ver que $\sigma$ não satisfaz a definição de um tempo de parada.

Proposição 2.9. Se a filtração for contínua à direita então $\tau$ é um tempo de parada se e somente se

$$
\{\omega \in \Omega: \tau(\omega)<t\} \in \mathscr{F}_{t}, \quad t \in \mathbb{R}_{+} .
$$

Demonstração. Se $\tau$ é um tempo de parada, então para $t \in \mathbb{R}_{+}$arbitrário,

$$
\{\tau<t\}=\bigcup_{n \in \mathbb{N}}\left\{\tau \leq t-\frac{1}{n}\right\} \in \mathscr{F}_{t}
$$

Reciprocamente, suponha que $\tau$ satisfaça (2.1) e a filtração seja contínua à direita. Então, para $t \in \mathbb{R}_{+}$arbitrário

$$
\{\tau \leq t\}=\bigcap_{k \in \mathbb{N}}\left\{\tau<t+\frac{1}{k}\right\}=\bigcap_{k \geq n}\left\{\tau<t+\frac{1}{k}\right\} \in \mathscr{F}_{t+\frac{1}{n}}, \quad \forall n \in \mathbb{N} .
$$

Assim,

$$
\{\tau \leq t\} \in \bigcap_{n \in \mathbb{N}} \mathscr{F}_{t+\frac{1}{n}}=\mathscr{F}_{t+}=\mathscr{F}_{t}
$$

Definição 2.10. Seja $\tau$ um tempo de parada. Denotamos a sub- $\sigma$-álgebra de $\mathscr{F}$ no tempo de parada $\tau$ por $\mathscr{F}_{\tau}$ e a definimos como a coleção de elementos de $\mathscr{F}_{\infty}$ da seguinte forma

$$
\mathscr{F}_{\tau} \doteq\left\{A \in \mathscr{F}_{\infty}: A \cap\{\tau \leq t\} \in \mathscr{F}_{t}, \forall t \in \mathbb{R}_{+}\right\}
$$

Proposição 2.11. Seja $\tau$ um tempo de parada. Então

i) $\mathscr{F}_{\tau}$ é uma $\sigma$-álgebra em $\Omega$,

ii) $\tau$ é $\mathscr{F}_{\tau}$-mensurável, 
iii) $\sigma(\tau) \subseteq \mathscr{F}_{\tau} \subseteq \mathscr{F}_{\infty} \subseteq \mathscr{F}$.

Demonstração. Para mostrar i, note em primeiro lugar que $\Omega \in \mathscr{F}_{\tau}$ pois $\Omega \cap\{\tau \leq t\}=\{\tau \leq t\} \in \mathscr{F}_{t}$, para todo $t \in \mathbb{R}_{+}$. Agora, sejam $A \in \mathscr{F}_{\tau}$ e $t \in \mathbb{R}_{+}$arbitrários. Temos

$$
A^{c} \cap\{\tau \leq t\}=[\Omega \cap\{\tau \leq t\}] \backslash[A \cap\{\tau \leq t\}]
$$

Como os dois conjuntos do lado direito estão em $\mathscr{F}_{t}$, a diferença também está e portanto $A^{c} \in \mathscr{F}_{\tau}$. Finalmente, sejam $\left\{A_{n}\right\} \in \mathscr{F}_{\tau}$ e $t \in \mathbb{R}_{+}$arbitrário. Como

$$
\left(\bigcup_{n \in \mathbb{N}} A_{n}\right) \cap\{\tau \leq t\}=\bigcup_{n \in \mathbb{N}}\left[A_{n} \cap\{\tau \leq t\}\right],
$$

resulta que $\cup_{n \in \mathbb{N}} A_{n} \in \mathscr{F}_{\tau}$.

Vamos provar ii: Como $\tau: \Omega \rightarrow \overline{\mathbb{R}}_{+}$, para provar que $\tau$ é $\mathscr{F}_{\tau}$-mensurável, basta provar que $\left\{\tau \leq t_{0}\right\} \in \mathscr{F}_{\tau}$ para todo $t_{0} \in \mathbb{R}_{+}$. Em primeiro lugar, $\left\{\tau \leq t_{0}\right\} \in \mathscr{F}_{\infty}$, além disso, se $t \geq t_{0}$, então

$$
\left\{\tau \leq t_{0}\right\} \cap\{\tau \leq t\}=\left\{\tau \leq t_{0}\right\} \in \mathscr{F}_{t_{0}} \subseteq \mathscr{F}_{t}
$$

Por outro lado, se $t<t_{0}$

$$
\left\{\tau \leq t_{0}\right\} \cap\{\tau \leq t\}=\{\tau \leq t\} \in \mathscr{F}_{t} .
$$

Assim, pela definição 2.10 resulta que $\left\{\tau \leq t_{0}\right\} \in \mathscr{F}_{\tau}$. Pela arbitrariedade de $t_{0}$, está provado o que queríamos.

Finalmente, para iii, note que como $\tau$ é $\mathscr{F}_{\tau}$-mensurável, $\sigma(\tau) \subseteq \mathscr{F}_{\tau}$. Pela definição de $\mathscr{F}_{\tau}$, $\mathscr{F}_{\tau} \subseteq \mathscr{F}_{\infty}$ e é imediato que $\mathscr{F}_{\infty} \subseteq \mathscr{F}$.

Definição 2.12. Seja $\mathbf{X}$ um processo estocástico. O instante de entrada em um conjunto $E \subseteq \mathbb{R}$ por $\mathbf{X}$ é uma função $T_{E}: \Omega \rightarrow \overline{\mathbb{R}}_{+}$dada por

$$
T_{E}(\omega)=\left\{\begin{array}{l}
\inf \left\{s \in \mathbb{R}_{+}: X(s, \omega) \in E\right\}, \\
\infty, \text { se }\left\{s \in \mathbb{R}_{+}: X(s, \omega) \in E\right\}=\emptyset .
\end{array}\right.
$$

Proposição 2.13. Sejam $\left(\Omega, \mathscr{F},\left\{\mathscr{F}_{t}\right\}, \mathrm{P}\right)$ um espaço de probabilidade filtrado contínuo à direita $e$ $\mathbf{X}$ um processo estocástico adaptado contínuo à direita ou à esquerda. Se $E \subseteq \mathbb{R}$ for um conjunto 
aberto, então o instante de entrada em E é um tempo de parada.

Demonstração. Suponha que $\mathbf{X}$ seja contínuo à direita. Como a filtração é contínua à direita, é suficiente mostrar que para $t \in \mathbb{R}_{+}$arbitrário vale que $\left\{\omega \in \Omega: T_{E}(\omega)<t\right\} \in \mathscr{F}_{t}$. Para $t=0$, $\left\{\omega \in \Omega: T_{E}(\omega)<t\right\}=\emptyset \in \mathscr{F}_{0}$. Agora, fixe $\omega_{0} \in \Omega$ e $t>0$. Temos que $T_{E}\left(\omega_{0}\right)<t$ se e somente se existir $s \in(0, t)$ tal que $X\left(s, \omega_{0}\right) \in E$. Como $\mathbf{X}$ é contínuo à direita e $E$ é aberto, $T_{E}\left(\omega_{0}\right)<t$ se e somente se, existir $r \in(0, t) \cap \mathbb{Q}$ tal que $X\left(r, \omega_{0}\right) \in E$. De fato, se existir tal $s$, por $E$ ser aberto e da continuidade de $\mathbf{X}$ existe $\delta>0$ tal que $X\left(t, \omega_{0}\right) \in E$ para $t \in(s-\delta, s+\delta)$. Da densidade dos racionais, $(s, s+\delta) \cap \mathbb{Q} \neq \emptyset$. Logo, $T(\omega)<t$ se e somente se, $\omega_{0} \in\{\omega \in \Omega: X(r, \omega) \in E\}$ para algum $r \in \mathbb{Q}, r<t$. Assim

$$
\left\{\omega \in \Omega: T_{E}(\omega)<t\right\}=\bigcup_{\substack{r<t \\ r \in \mathbb{Q}}}\{\omega \in \Omega: X(r, \omega) \in E\} \in \mathscr{F}_{t}
$$

e portanto, segue que $T_{E}$ é tempo de parada. Para $\mathbf{X}$ contínuo à esquerda a prova é análoga.

Deste resultado, segue imediatamente o seguinte

Corolário 2.14. Sejam $\left(\Omega, \mathscr{F},\left\{\mathscr{F}_{t}\right\}, \mathrm{P}\right)$ um espaço de probabilidade filtrado contínuo à direita e $\mathbf{X}$ um processo estocástico adaptado contínuo à direita ou à esquerda. Então, para qualquer c $\geq 0$

$$
T_{c} \doteq \inf \{t: X(t, \cdot)>c\}
$$

é um tempo de parada.

Proposição 2.15. Sejam $\mathbf{X}$ um processo estocástico adaptado e contínuo e $B \subseteq \mathbb{R}$ um conjunto fechado. Então o instante de entrada em B é um tempo de parada.

Demonstração. Como as trajetórias são contínuas, para cada $\omega \in \Omega$, os conjuntos $X([0, t], \omega)$ são compactos. Pelo fato de $B$ ser fechado, $X([0, t], \omega) \cap B=\emptyset$ se e somente se $d(X([0, t], \omega), B)>0$, ou 
seja, se e somente se $T_{B}(\omega)>t$. Assim,

$$
\begin{aligned}
\left\{\omega \in \Omega: T_{B}(\omega) \leq t\right\} & =\{\omega \in \Omega: X([0, t], \omega) \cap B \neq \emptyset\} \\
& =\{\omega \in \Omega: d(X([0, t], \omega), B)=0\} \\
& =\{\omega \in \Omega: \inf \{d(X(s, \omega), B), s \leq t\}=0\} \\
& =\{\omega \in \Omega: \inf \{d(X(s, \omega), B), s \leq t, s \in \mathbb{Q}\}=0\},
\end{aligned}
$$

onde a última igualdade vale pois $\mathbf{X}$ é contínuo e $X([0, t] \cap \mathbb{Q}, \omega)$ é denso em $X([0, t], \omega)$. Agora, temos que $X(s, \omega)$ é $\mathscr{F} t$-mensurável para todo $s \leq t$ e como $x \mapsto d(x, B)$ é contínua, $d(X(s, \omega), B)$ também é $\mathscr{F} t$-mensurável e pelo fato do ínfimo de uma quantidade enumerável de funções mensuráveis ser mensurável, segue que $\left\{\omega \in \Omega: T_{B}(\omega) \leq t\right\} \in \mathscr{F}_{t}$, ou seja, $T_{B}$ é tempo de parada.

No próximo teorema listamos algumas propriedades básicas dos tempos de parada. Para a demonstração destes fatos, veja por exemplo [27].

Teorema 2.16. Sejam $c \in \mathbb{R}_{+}$e $\tau$ e $\sigma$ tempos de parada definidos no mesmo espaço filtrado de probabilidade. Então

i) $\tau \wedge \sigma, \tau \vee \sigma$ e $\tau+c$, são tempos de parada.

ii) Para cada $t \in \mathbb{R}_{+}, \sigma \wedge t$ é $\mathscr{F}_{t}$-mensurável.

iii) Se $\sigma \leq \tau$ em $\Omega$, então $\mathscr{F}_{\sigma} \subseteq \mathscr{F}_{\tau}$.

Corolário 2.17. Sejam $\tau$ um tempo de parada e $\mathbf{X}$ um processo estocástico adaptado à $\left\{\mathscr{F}_{\tau \wedge t}\right\}$. Então $\mathbf{X}$ é adaptado à $\left\{\mathscr{F}_{t}\right\}$.

Demonstração. Basta notar que $\tau \wedge t \leq t$ e portanto pelo resultado anterior, $\mathscr{F}_{\tau \wedge t} \subseteq \mathscr{F}_{t}$.

Definição 2.18. Sejam $\mathbf{X}$ um processo estocástico e $\tau$ um tempo de parada.

i) Definimos o processo truncado em $\tau$ por

$$
X^{\tau}(t, \omega) \doteq X(\tau(\omega) \wedge t, \omega)
$$


ii) Definimos a variável aleatória parada em $\tau$ como

$$
X_{\tau}(\omega) \doteq\left\{\begin{array}{l}
X(\tau(\omega), \omega), \text { se } \tau(\omega)<\infty \\
0, \text { se } \tau(\omega)=\infty
\end{array}\right.
$$

Seja $\mathbf{X}$ um processo estocástico adaptado. Gostaríamos que $X_{\tau}$ fosse $\mathscr{F}_{\tau}$-mensurável. Esse é um dos principais motivos para introduzir o conceito de mensurabilidade progressiva que é o tema da próxima seção.

\subsection{Mensurabilidade progressiva}

Definição 2.19. Dizemos que um processo estocástico $\mathbf{X}$ é progressivamente mensurável com respeito $\grave{a}$ filtração $\left\{\mathscr{F}_{t}\right\}_{t \geq 0}$, quando para cada $t \in \mathbb{R}_{+}$, a restrição de $\mathbf{X}$ para $[0, t] \times \Omega$ for uma aplicação $\sigma\left(\mathscr{B}_{[0, t]} \times \mathscr{F}_{t}\right) / \mathscr{B}_{\mathbb{R}}$-mensurável de $[0, t] \times \Omega$ em $\mathbb{R}$. Diremos que um subconjunto $\Gamma \subseteq \mathbb{R}_{+} \times \Omega$ é progressivo quando $\mathbb{1}_{\Gamma}$ for um processo progressivamente mensurável. Denotaremos a familía de todos os subconjuntos progressivos de $\mathbb{R}_{+} \times \Omega$ por $\mathfrak{P}_{g}$.

Observamos que $\mathfrak{P}_{g}$ é uma $\sigma$-álgebra em $\mathbb{R}_{+} \times \Omega$.

Definição 2.20. Dizemos que um processo estocástico $\mathbf{X}$ é mensurável quando $\mathbf{X}: \mathbb{R}_{+} \times \Omega \rightarrow \mathbb{R}$ for

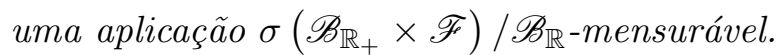

Sabemos da teoria de medida, que se $\mathbf{X}$ é mensurável, então para qualquer $\omega \in \Omega, X(\cdot, \omega)$ é mensurável com respeito a $\mathscr{B}_{\mathbb{R}}$. Em outras palavras, toda trajetória de um processo mensurável é Borel mensurável.

Os próximos dois resultados apresentam caracterizações dos processos progressivamente mensuravéis.

Proposição 2.21. Seja $\mathbf{X}$ um processo progressivamente mensurável. Então $\mathbf{X}$ é adaptado e mensurável.

Demonstração. Vamos provar inicialmente a adaptabilidade de $\mathbf{X}$. Como $\mathbf{X}$ é progressivamente mensurável, para cada $t \in \mathbb{R}_{+}$a restrição de $\mathbf{X}$ para $[0, t] \times \Omega$ é uma aplição $\sigma\left(\mathscr{B}_{[0, t]} \times \mathscr{F}_{t}\right) / \mathscr{B}_{\mathbb{R}^{-}}$ mensurável de $[0, t] \times \Omega$ em $\mathbb{R}$. Assim, $X(s, \cdot)$ é $\mathscr{F}_{t}$-mensurável para cada $s \in[0, t]$. Em particular, $X(t, \cdot)$ é $\mathscr{F}_{t}$-mensurável e portanto $\mathbf{X}$ é um processo $\left\{\mathscr{F}_{t}\right\}$-adaptado. 
Agora vamos provar que $\mathbf{X}$ é um processo mensurável. Como $\mathbf{X}$ é progressivamente mensurável, então para cada $n \in \mathbb{N}$, a restrição de $\mathbf{X}$ para $[0, n] \times \Omega$ é uma aplicação $\sigma\left(\mathscr{B}_{[0, n]} \times \mathscr{F}_{n}\right) / \mathscr{B}_{\mathbb{R}^{-}}$ mensurável de $[0, n] \times \Omega$ em $\mathbb{R}$ e portanto também é uma aplicação $\sigma\left(\mathscr{B}_{[0, n]} \times \mathscr{F}_{\infty}\right) / \mathscr{B}_{\mathbb{R}}$-mensurável de $[0, n] \times \Omega$ em $\mathbb{R}$. Dessa maneira, $\mathbf{X} \cdot \mathbb{1}_{[0, n] \times \Omega}$ é uma aplicação $\sigma\left(\mathscr{B}_{\mathbb{R}_{+}} \times \mathscr{F}_{\infty}\right) / \mathscr{B}_{\mathbb{R}}$ de $\mathbb{R}_{+} \times \Omega$ em $\mathbb{R}$. Dessa forma, como $\mathbf{X}=\lim _{n \rightarrow \infty} \mathbf{X} \cdot \mathbb{1}_{[0, n] \times \Omega}$, segue que $\mathbf{X}$ é uma aplicação $\sigma\left(\mathscr{B}_{\mathbb{R}_{+}} \times \mathscr{F}_{\infty}\right) / \mathscr{B}_{\mathbb{R}}$ de $\mathbb{R}_{+} \times \Omega$ em $\mathbb{R}$. Isto é, $\mathbf{X}$ é um processo mensurável.

Proposição 2.22. Seja $\mathbf{X}$ um processo adaptado, contínuo à direita ou à esquerda. Então $\mathbf{X}$ é progressivamente mensurável.

Demonstração. Suponha inicialmente $\mathbf{X}$ contínuo à esquerda e adaptado. Para $t \in \mathbb{R}_{+}$fixo, defina

$$
I_{n, k} \doteq\left(\frac{k-1}{2^{n}} t, \frac{k}{2^{n}} t\right]
$$

para $k=1, \ldots, 2^{n}, n \in \mathbb{N}$ e considere a sequência de processos contínuos à esquerda $\left\{\mathbf{X}^{(n)}\right\}_{n \in \mathbb{N}}$ definidos em $[0, t] \times \Omega$ por

$$
X^{(n)}(s, \omega) \doteq\left\{\begin{array}{l}
X(0, \omega), \text { se } s=0, \omega \in \Omega \\
X\left(\frac{k-1}{2^{n}} t, \omega\right), \text { se } s \in I_{n, k}, \omega \in \Omega
\end{array}\right.
$$

Afirmamos que $\lim _{n \rightarrow \infty} X^{(n)}(s, \omega)=X(s, \omega)$ para $(s, \omega) \in[0, t] \times \Omega$. De fato, perceba inicialmente que $X^{(n)}(0, \cdot)=X(0, \cdot)$ para todo $n \in \mathbb{N}$, logo basta provar para $(s, \omega) \in(0, t] \times \Omega$. Agora, para cada $n \in \mathbb{N}$, seja $k_{n} \in \mathbb{N}$ o único índice tal que $s \in I_{n, k_{n}}$. Temos portanto que $X^{(n)}(s, \omega)=X\left(\frac{k_{n}-1}{2^{n}} t, \omega\right)$. Agora, como para todo $n \in \mathbb{N}$ vale que

$$
\frac{\left(k_{n}-1\right)}{2^{n}} t<s
$$

$\mathrm{e}$

$$
s-\frac{\left(k_{n}-1\right)}{2^{n}} t<\frac{t}{2^{n}},
$$

temos que

$$
\lim _{n \rightarrow \infty} \frac{\left(k_{n}-1\right)}{2^{n}} t \uparrow s .
$$


Assim, como $\mathbf{X}$ é contínuo à esquerda

$$
\lim _{n \rightarrow \infty} X^{(n)}(s, \omega)=\lim _{n \rightarrow \infty} X\left(\frac{k_{n}-1}{2^{n}} t, \omega\right)=X(s, \omega) .
$$

Agora, provada a convergência de $\mathbf{X}^{(n)}$ a $\mathbf{X}$ em $[0, t] \times \Omega$, basta provar que para cada $n \in \mathbb{N}, \mathbf{X}^{(n)}$ é $\sigma\left(\mathscr{B}_{[0, t]} \times \mathscr{F}_{t}\right)$-mensurável e o resultado segue pois o limite de aplições mensuráveis é mensurável e assim a restrição de $\mathbf{X}$ para $[0, t] \times \Omega$ é $\sigma\left(\mathscr{B}_{[0, t]} \times \mathscr{F}_{t}\right)$-mensurável. Para isto, fixe $n \in \mathbb{N}$ e seja $A \in \mathscr{B}_{\mathbb{R}}$ um boreliano arbitrário. Temos que

$$
\left(X^{(n)}\right)^{-1}(A)=\left(\{0\} \times(X(0, \cdot))^{-1}(A)\right) \cup\left[\bigcup_{k=1, \ldots, 2^{n}}\left(I_{n, k} \times\left(X\left(\frac{k-1}{2^{n}} t, \cdot\right)\right)^{-1}(A)\right)\right] .
$$

Como X é $\left\{\mathscr{F}_{t}\right\}$-adaptado, $(X(0, \cdot))^{-1}(A) \in \mathscr{F}_{0} \subseteq \mathscr{F}_{t}$ e $\left(X\left(\frac{k-1}{2^{n}} t, \cdot\right)\right)^{-1}(A) \in \mathscr{F}_{\frac{k-1}{2^{n}} t} \subseteq \mathscr{F}_{t}$ para todo $k=1, \ldots, 2^{n}$. Além disso, $\{0\}$ e $I_{n, k}$ são $\mathscr{B}_{[0, t]}$-mensuráveis para todo $k=1, \ldots, 2^{n}$. Resulta portanto que $\left(X^{(n)}\right)^{-1}(A)$ como união enumerável de elmentos $\mathscr{B}_{[0, t]} \times \mathscr{F}_{t}$-mensuráveis é $\sigma\left(\mathscr{B}_{[0, t]} \times \Omega\right)$ mensurável. Da arbitrariedade de $A \in \mathscr{B}_{\mathbb{R}}$ resulta que $\mathbf{X}^{(n)}$ é $\sigma\left(\mathscr{B}_{[0, t]} \times \mathscr{F}_{t}\right)$-mensurável, provando o caso $\mathbf{X}$ contínuo à esquerda.

Para o caso $\mathbf{X}$ contínuo à direita, tome

$$
I_{n, k} \doteq\left[\frac{k-1}{2^{n}} t, \frac{k}{2^{n}} t\right)
$$

para $k=1, \ldots, 2^{n}$ e $n \in \mathbb{N}$ e considere a sequência de processos contínuos à direita $\left\{\mathbf{X}^{(n)}\right\}_{n \in \mathbb{N}}$ definidos em $[0, t] \times \Omega$ por

$$
X^{(n)}(s, \omega) \doteq X\left(\frac{k}{2^{n}} t, \omega\right) \text { se } s \in I_{n, k} \text { e } \omega \in \Omega
$$

Com essas alterações a demonstração para este caso é análoga ao caso anterior.

Agora, vamos apresentar o resultado pelo qual o conceito de mensurabilidade progressiva foi introduzido.

Teorema 2.23. Seja $\mathbf{X}$ um processo progressivamente mensurável e $\tau$ um tempo de parada. Então o processo truncado $\mathbf{X}^{\tau}$ é progressivamente mensurável e portanto, em particular adaptado e a variável 
aleatória parada $X_{\tau}$ é $\mathscr{F}_{\tau}$-mensurável.

Demonstração. Fixe $t \geq 0$. Considere as aplicações

$$
\begin{aligned}
u:\left([0, t] \times \Omega, \sigma\left(\mathscr{B}_{[0, t]} \times \mathscr{F}_{t}\right)\right) & \rightarrow\left([0, t], \mathscr{B}_{[0, t]}\right) \\
(s, \omega) & \mapsto \tau(\omega) \wedge s \\
v:\left([0, t] \times \Omega, \sigma\left(\mathscr{B}_{[0, t]} \times \mathscr{F}_{t}\right)\right) & \rightarrow\left(\Omega, \mathscr{F}_{t}\right) \\
(s, \omega) & \mapsto \omega .
\end{aligned}
$$

Temos evidentemente que $u$ é $\sigma\left(\mathscr{B}_{[0, t]} \times \mathscr{F}_{t}\right) / \mathscr{B}_{[0, t]}$-mensurável e $v$ é $\sigma\left(\mathscr{B}_{[0, t]} \times \mathscr{F}_{t}\right) / \mathscr{F}_{t}$-mensurável e portanto a aplicação

$$
\begin{aligned}
h:\left([0, t] \times \Omega, \sigma\left(\mathscr{B}_{[0, t]} \times \mathscr{F}_{t}\right)\right) & \rightarrow\left([0, t] \times \Omega, \sigma\left(\mathscr{B}_{[0, t]} \times \mathscr{F}_{t}\right)\right) \\
(s, \omega) & \mapsto(u(s, \omega), v(s, \omega)=(\tau(\omega) \wedge s, \omega)
\end{aligned}
$$

é $\sigma\left(\mathscr{B}_{[0, t]} \times \mathscr{F}_{t}\right) / \sigma\left(\mathscr{B}_{[0, t]} \times \mathscr{F}_{t}\right)$-mensurável. Além disso, por ser progressivamente mensurável, $\mathbf{X}$

$$
\begin{aligned}
\mathbf{X}:\left([0, t] \times \Omega, \sigma\left(\mathscr{B}_{[0, t]} \times \mathscr{F}_{t}\right)\right) & \rightarrow\left(\overline{\mathbb{R}}, \mathscr{B}_{\overline{\mathbb{R}}}\right) \\
(s, \omega) & \mapsto X(s, \omega)
\end{aligned}
$$

é uma aplicação $\sigma\left(\mathscr{B}_{[0, t]} \times \mathscr{F}_{t}\right) / \mathscr{B}_{\mathbb{R}^{-}}$mensurável. Assim, como $\mathbf{X}^{\tau}=\mathbf{X} \circ h$, isto é,

$$
\begin{aligned}
\mathbf{X}^{\tau}:\left([0, t] \times \Omega, \sigma\left(\mathscr{B}_{[0, t]} \times \mathscr{F}_{t}\right)\right) & \rightarrow\left(\overline{\mathbb{R}}, \mathscr{B}_{\overline{\mathbb{R}}}\right) \\
(s, \omega) & \mapsto X(\tau(\omega) \wedge s, \omega)=X^{\tau}(s, \omega),
\end{aligned}
$$

segue que $\mathbf{X}^{\tau}$ é $\sigma\left(\mathscr{B}_{[0, t]} \times \mathscr{F}_{t}\right) / \mathscr{B}_{\mathbb{R}^{-}}$mensurável. Da arbitrariedade de $t$ segue que $\mathbf{X}^{\tau}$ é progressivamente mensurável e em particular é adaptado.

Vamos provar agora que a variável parada $X_{\tau}$ é $\mathscr{F}_{\tau}$-mensurável. Seja $A \in \mathscr{B}_{\overline{\mathbb{R}}}$ um boreleano arbitrário. Devemos mostrar que $\left(X_{\tau}\right)^{-1}(A) \in \mathscr{F}_{\tau}$, pela definição 2.10, isso quer dizer que devemos 
provar que $\left(X_{\tau}\right)^{-1}(A) \cap\{\tau \leq t\} \in \mathscr{F} t$ para todo $t \in \mathbb{R}_{+}$. De fato,

$$
\begin{aligned}
\left\{X_{\tau} \in A\right\} \cap\{\tau \leq t\} & =\{X(\tau(\omega) \wedge t, \omega) \in A\} \cap\{\tau \leq t\} \\
& =\left\{X^{\tau}(t, \omega) \in A\right\} \cap\{\tau \leq t\}
\end{aligned}
$$

Como $\mathbf{X}^{\tau}$ é adaptado e $\tau$ é tempo de parada, $\left\{X_{\tau} \in A\right\} \cap\{\tau \leq t\} \in \mathscr{F}_{t}$ e está provado o resultado.

Definição 2.24. Dizemos que um espaço de probabilidade filtrado $\left(\Omega, \mathscr{F},\left\{\mathscr{F}_{t}\right\}, \mathrm{P}\right)$ satisfaz as condições usuais quando

i) $\left\{\mathscr{F}_{t}\right\}$ é contínua à direita.

ii) $\left\{\mathscr{F}_{t}\right\}$ é aumentada, isto é, $\mathscr{F}_{0}$ (e portanto $\mathscr{F}_{t}$, para todo $t \geq 0$ ) contém os conjuntos de medida P-nula.

iii) O espaço $(\Omega, \mathscr{F}, \mathrm{P})$ é completo.

Já vimos algumas vantagens de trabalhar com filtrações contínuas à direita para tempos de entrada. A condição da filtração ser aumentada elimina alguns problemas de mensurabilidade. De fato, se $\mathbf{X}$ é adaptado e $\mathbf{Y}$ é uma modificação de $\mathbf{X}$ então segue que $\mathbf{Y}$ também é adaptado. $\mathrm{O}$ mesmo resultado vale para indistinguibilidade. Além disso, se $\left\{X_{n}\right\}_{n \in \mathbb{N}}$ é uma sequência de funções

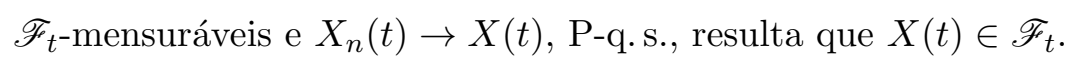

\subsection{Martingais}

Definição 2.25. Dizemos que um processo estocástico $\mathbf{X}$ adptado à $\left\{\mathscr{F}_{t}\right\}_{t \geq 0}$ é um submartingal com respeito à $\left\{\mathscr{F}_{t}\right\}_{t \geq 0}$ quando

i) $\mathbf{E}\left(X_{t}^{+}\right)<\infty$, para todo $t \in \Theta$.

ii) $\mathbf{E}\left(X_{t} \| \mathscr{F}_{s}\right) \geq X_{s}$ para quase todo $\omega \in \Omega$ e todo $s \leq t$.

Se - $\mathbf{X}$ for um submartingal, dizemos que $\mathbf{X}$ é um supermartingal e se $\mathbf{X}$ for um submartingal $e$ supermartingal, dizemos que $\mathbf{X}$ é um martingal.

Observe que segue pela definição que se $\mathbf{X}$ é um martingal então para cada $t \in \Theta, \mathbf{E}\left(X_{t}\right)<\infty$, isto é, $X_{t}$ é integrável para cada $t$. Observamos também que se $\mathbf{X}$ é um martingal, então, pela 
desigualdade de Jensen* e propriedades da esperança condicional ${ }^{\dagger}$, para $p \geq 1$ e $s \leq t$, vale que

$$
\mathbf{E}\left(\left|X_{t}\right|^{p}\right)=\mathbf{E}\left(\mathbf{E}\left(\left|X_{t}\right|^{p}|| \mathscr{F}_{s}\right)\right) \geq \mathbf{E}\left(\left|\mathbf{E}\left(X_{t}|| \mathscr{F}_{s}\right)\right|^{p}\right)=\mathbf{E}\left(\left|X_{s}\right|^{p}\right)
$$

Assim, temos que $\sup _{t \geq 0}\left\|X_{t}\right\|_{p}=\lim _{t \rightarrow \infty}\left\|X_{t}\right\|_{p}$.

Se interpretarmos $X_{t}$ como o capital de um jogador no instante $t$, então um martingal pode ser pensado como um modelo de um jogo honesto no sentido de que toda informação disponível em um instante $s$ não muda o fato de que o incremento esperado do capital durante o intervalo de tempo $[s, t]$ é igual a zero, em símbolos: $\mathbf{E}\left(X_{t}-X_{s} \| \mathscr{F}_{s}\right)=0$.

Exemplo 2.26 (Movimento Browniano padrão). Um exemplo de martingal é o movimento Browniano padrão B. De fato, seja $0 \leq s \leq t$, então

$$
\begin{aligned}
\mathbf{E}\left(B(t) \| \mathscr{F}_{s}\right) & =\mathbf{E}\left(B(s)+B(t)-B(s) \| \mathscr{F}_{s}\right) \\
& =\mathbf{E}\left(B(s) \| \mathscr{F}_{s}\right)+\mathbf{E}\left(B(t)-B(s) \| \mathscr{F}_{s}\right) \\
& =B(s)+\mathbf{E}\left(B(t)-B(s) \| \mathscr{F}_{s}\right) \\
& =B(s)+\mathbf{E}(B(t)-B(s)) \\
& =B(s),
\end{aligned}
$$

onde a quarta igualdade vale pelo teorema C.12 e a quinta pelo fato dos incrementos do movimento Browniano ter média 0 .

As demonstrações dos próximos quatro resultados dependem da introdução de alguns conceitos que nos desviariam do foco deste trabalho. O leitor interessado pode consultar [29].

Teorema 2.27 (Desigualdade de Doob). Seja $\mathbf{X}$ um martingal contínuo à direita ou um supermartingal positivo. Defina $X^{*}=\sup _{t}\left|X_{t}\right|$ e seja $t_{d}$ o extremo direito de $\Theta$. Então

i) $\lambda^{p} \mathrm{P}\left(X^{*} \geq \lambda\right) \leq \sup _{t} \mathbf{E}\left(\left|X_{t}\right|^{p}\right)$, se $p \geq 1$.

ii) $\left\|X^{*}\right\|_{p} \leq \frac{p}{1-p} \sup _{t}\left\|X_{t}\right\|_{p}$, se $p>1$.

iii) $\left\|X^{*}\right\|_{p} \leq \frac{p}{1-p} \lim _{t \uparrow t_{d}}\left\|X_{t}\right\|_{p}$, se $p>1$ e $\Theta$ é aberto à direita.

iv) $\left\|X^{*}\right\|_{p} \leq \frac{p}{1-p}\left\|X_{t_{d}}\right\|_{p}$, se $p>1$ e $\Theta$ é fechado à direita.

* Veja o teorema C.13 na página $162 . \quad$ † Veja o teorema C.10 na página 160. 
Tendo em vista este importante teorema, gostaríamos de que todos nossos martingais fossem contínuos à direita. Felizmente, as condições usuais nos permitem sempre selecionar uma modificação contínua à direita. Alías, até um pouco mais do que isso.

Definição 2.28. Dizemos que um processo estocástico $\mathbf{X}$ é càdlàg (do frânces continue à droite, limitée à gauche) quando for contínuo à direita e com limites (finitos) à esquerda. Dizemos que $\mathbf{X}$ é càglàd (do francês continue à gauche, limitée à droite) quando for contínuo à esquerda e com limites (finitos) à direita.

Teorema 2.29. Se estamos sob as condições usuais, então todo martingal $\mathbf{X}$ admite um modificação que é càdlàg.

Tendo em vista esse resultado, de agora em diante, estaremos assumindo tacitamente que todos os martingais são càdlàg. Observamos que é possível* mostrar que a $\sigma$-álgebra aumentada $\left\{\overline{\mathscr{F}}_{t}^{\mathrm{B}}\right\}_{t}$ de um movimento Browniano $\mathbf{B}$ é contínua à direita.

Vamos agora relacionar martingais com o conceito de integrabilidade uniforme ${ }^{\dagger}$. Faremos antes uma pequena digressão para lembrar o leitor das desigualdades de Markov e Chebyshev. As demonstrações dos próximos três resultados podem ser encontradas em [14].

Teorema 2.30. Seja $f$ uma função não negativa, mensurável no espaço $(\Omega, \mathscr{F}, \mu)$. Então, para $t \in(0, \infty)$,

$$
\mu(\{f \geq t\}) \leq \frac{\int_{\Omega} f d \mu}{t}
$$

O seguinte corolário é conhecido como a Desigualdade de Markov.

Corolário 2.31. Seja $X$ uma variavél aleatória definida em $(\Omega, \mathscr{F}, \mathrm{P})$. Então, para $r, t>0$,

$$
\mathrm{P}(|X| \geq t) \leq \frac{\mathbf{E}\left(|X|^{r}\right)}{t^{r}}
$$

A próxima desigualdade é a Desigualdade de Chebyshev.

Corolário 2.32. Seja $X$ uma variavél aleatória definida em $(\Omega, \mathscr{F}, \mathrm{P})$ tal que $\mathbf{E}\left(X^{2}\right)<\infty$. Então, para qualquer $k \in(0, \infty)$,

$$
\mathrm{P}(|X-\mathbf{E}(X)| \geq k) \leq \frac{\operatorname{Var}(X)}{k^{2}}
$$

\footnotetext{
* Veja por exemplo [15], página 67. † Veja a definição B.4 na página 154.
} 
O próximo importante lema será usado em diversas ocasiões neste trabalho.

Lema 2.33. Seja $\xi \in \mathrm{L}^{1}$ e $\left\{\mathscr{F}_{\alpha}\right\}_{\alpha \in A}$ uma família de $\sigma$-álgebras. Então a família de variavéis aleatórias $\left\{X_{\alpha}\right\}_{\alpha \in A}$ definidas como

$$
X_{\alpha} \doteq \mathbf{E}\left(\xi \| \mathscr{F}_{\alpha}\right)
$$

é uniformemente integrável.

Demonstração. Pelas desigualdades de Markov e de Jensen temos, para $\alpha \in A$ arbitrário, que

$$
\begin{aligned}
\mathrm{P}\left(\left|X_{\alpha}\right| \geq n\right) & \leq \frac{\mathbf{E}\left(\left|X_{\alpha}\right|\right)}{n} \\
& =\frac{\mathbf{E}\left(\left|\mathbf{E}\left(\xi|| \mathscr{F}_{\alpha}\right)\right|\right)}{n} \\
& \leq \frac{\mathbf{E}\left(\mathbf{E}\left(|\xi||| \mathscr{F}_{\alpha}\right)\right)}{n} \\
& =\frac{\mathbf{E}(|\xi|)}{n}
\end{aligned}
$$

Assim, para qualquer $\delta>0$, existe $n_{0} \in \mathbb{N}$ tal que se $n>n_{0}, \mathrm{P}\left(\left|X_{\alpha}\right| \geq n\right)<\delta$. Como $\xi \in \mathrm{L}^{1} \mathrm{e}$ a integral de Lebesgue é absolutamente contínua com respeito a medida de Lebesgue, dado $\varepsilon>0$, existe $\delta>0$ tal que se $\mathrm{P}(A)<\delta$, então $\int_{A}|\xi| d \mathrm{P}<\varepsilon$. Assim, para $n$ suficientemente grande,

$$
\int_{\left|X_{\alpha}\right|>n}\left|X_{\alpha}\right| d \mathrm{P} \leq \int_{\left|X_{\alpha}\right|>n} \mathbf{E}\left(|\xi||| \mathscr{F}_{\alpha}\right) d \mathrm{P}=\int_{\left|X_{\alpha}\right|>n}|\xi| d \mathrm{P}<\varepsilon
$$

onde a última igualdade vem da definição da esperança condicional. Dessa forma, provamos que $\left\{X_{\alpha}\right\}_{\alpha \in A}$ é uniformemente integrável.

Definição 2.34. Seja $p \in \mathbb{N}$. Dizemos que um martingal $\mathbf{X}$ é limitado em $\mathrm{L}^{p}$ quando $\sup _{t} \mathbf{E}\left(\left|X_{t}\right|^{p}\right)<\infty$.

As demonstrações dos próximos importantes resultados podem ser encontrados em [23].

Teorema 2.35 (Convergência de Doob). Seja $\mathbf{X}$ um martingal. São equivalentes

i) $\lim _{t \rightarrow \infty} X_{t}$ existe em $\mathrm{L}^{1}$.

ii) Existe uma variável aleatória $X_{\infty} \in \mathrm{L}^{1}$ tal que $X_{t}=\mathbf{E}\left(X_{\infty} \| \mathscr{F}_{t}\right)$ para todo $t \geq 0$. 
iii) A familia $\left\{X_{t}: t \in \mathbb{R}_{+}\right\}$é uniformemente integrável.

Se essas condições forem satisfeitas, então $X_{\infty}=\lim _{t \rightarrow \infty} X_{t}$ q. s. Além disso, se para algum $p>1$, o martingal for limitado em $\mathrm{L}^{p}$, então as condiçôes acima estão satisfeitas e a convergência vale em $\mathrm{L}^{p}$.

O próximo resultado pode ser entendido intuitivamente pensando num martingal como um modelo de jogo honesto. Em um jogo honesto, um apostador não pode melhorar ou piorar o valor esperado do seu capital entrando no jogo em um instante de tempo $\tau_{1}(\omega)$ e saindo do jogo em $\tau_{2}(\omega)$ dado que ele só pode decidir os instantes de entrada e saida do jogo baseado apenas nas informações disponíveis no instante da decisão (ou seja, sem 'olhar' no futuro).

Teorema 2.36 (Doob's Optional Sampling). Sejam $\mathbf{X}$ um martingal e $\tau_{1} \leq \tau_{2}$ tempos de parada. Se $\mathbf{X}$ for uniformemente integrável ou $\tau_{1}$ e $\tau_{2}$ forem limitados, então para quase todo $\omega \in \Omega$,

$$
X\left(\tau_{1}\right)=\mathbf{E}\left(X\left(\tau_{2}\right) \| \mathscr{F}_{\tau_{1}}\right) .
$$

Proposição 2.37 (Doob's Optional Stopping). Seja $\mathbf{X}$ um processo càdlàg e adaptado. Então $\mathbf{X}$ é um martingal se e somente se

$$
X(\tau) \in \mathrm{L}^{1} \text { e } \mathbf{E}(X(\tau))=\mathbf{E}(X(0))
$$

para qualquer tempo de parada limitado $\tau$. Além disso, (2.2) vale para qualquer tempo de parada se e somente se $\mathbf{X}$ for um martingal uniformemente integrável.

Demonstração. De fato, se $\mathbf{X}$ é um martingal ou um martingal uniformemente integrável, pelo teorema anterior,

$$
\mathbf{E}(X(0))=\mathbf{E}\left(\mathbf{E}\left(X(\tau) \| \mathscr{F}_{0}\right)\right)=\mathbf{E}(X(\tau))
$$

Reciprocamente, sejam $s<t$ e $A \in \mathscr{F}_{s}$. Defina

$$
\tau \doteq t \mathbb{1}_{A^{c}}+s \mathbb{1}_{A}
$$

Temos que $\tau$ é um tempo de parada limitado. De fato, temos para $r \in \mathbb{R}_{+}$que

$$
\{\omega \in \Omega: \tau(\omega) \leq r\}=\left[\left\{\omega \in A^{c}\right\} \cap\{\omega \in \Omega: t \leq r\}\right] \bigcup[\{\omega \in A\} \cap\{\omega \in \Omega: s \leq r\}] .
$$


Assim, se $s<t \leq r$, então $\{\tau \leq r\}=\Omega \in \mathscr{F}_{r}$, se $s \leq r<t$, então $\{\tau \leq r\}=A \in \mathscr{F}_{s} \subseteq \mathscr{F}_{r}$ e finalmente se $r<s<t$ segue que $\{\tau \leq r\}=\emptyset \in \mathscr{F}_{r}$. Assim, por hipótese, temos que

$$
\mathbf{E}(X(0))=\mathbf{E}(X(\tau))=\mathbf{E}\left(X(t) \mathbb{1}_{A^{c}}\right)+\mathbf{E}\left(X(s) \mathbb{1}_{A}\right)
$$

Porém, como $\tau \equiv t$ também é um tempo de parada

$$
\mathbf{E}(X(0))=\mathbf{E}(X(t))=\mathbf{E}\left(X(t) \mathbb{1}_{A^{c}}\right)+\mathbf{E}\left(X(s) \mathbb{1}_{A}\right)
$$

e portanto, $\mathbf{E}\left(X(t) \mathbb{1}_{A}\right)=\mathbf{E}\left(X(s) \mathbb{1}_{A}\right)$. Como $A \in \mathscr{F}_{s}$ é arbitrário,

$$
\mathbf{E}\left(X(s) \| \mathscr{F}_{s}\right)=\mathbf{E}\left(X(t) \| \mathscr{F}_{s}\right) .
$$

Como $\mathbf{X}$ é adaptado, $X(s)$ é $\mathscr{F}_{s}$-mensurável e pelo teorema C.8, $X(s)=\mathbf{E}\left(X(s) \| \mathscr{F}_{s}\right)$ e portanto

$$
X(s)=\mathbf{E}\left(X(t) \| \mathscr{F}_{s}\right)
$$

o que mostra que $\mathbf{X}$ é martingal. Agora, se (2.2) valer para qualquer tempo de parada, então em particular, vale para $\tau \equiv \infty$. Resulta que $X(\infty)=X_{\infty}$ é integrável. Assim, definindo

$$
\sigma \doteq \infty \mathbb{1}_{A^{c}}+s \mathbb{1}_{A}
$$

temos

$$
\mathbf{E}(X(\sigma))=\mathbf{E}\left(X_{\infty} \mathbb{1}_{A^{c}}\right)+\mathbf{E}\left(X(s) \mathbb{1}_{A}\right)=\mathbf{E}(X(0))
$$

$\mathrm{e}$

$$
\mathbf{E}(X(\infty))=\mathbf{E}\left(X_{\infty} \mathbb{1}_{A^{c}}\right)+\mathbf{E}\left(X_{\infty} \mathbb{1}_{A}\right)=\mathbf{E}(X(0)) .
$$

Assim, como antes, ficamos com

$$
X(s)=\mathbf{E}\left(X_{\infty} \| \mathscr{F}_{s}\right),
$$

e portanto pelo lema 2.33, resulta que $\mathbf{X}$ é uniformemente integrável.

Corolário 2.38. Se $\mathbf{X}$ é um martingal e $\tau$ um tempo de parada então o processo truncado $\mathbf{X}^{\tau}$ também é um martingal.

Demonstração. Pelo teorema 2.23, segue que $\mathbf{X}^{\tau}$ é adaptado. Agora, seja $\phi$ um tempo de parada 
limitado e defina $v=\phi \wedge \tau$. Temos pelo teorema 2.16 que $v$ é tempo de parada e é obviamente limitado. Agora pela definição de processo parado e $v$,

$$
\mathbf{E}\left(X^{\tau}(\phi)\right)=\mathbf{E}(X(v))
$$

Mas, como $\mathbf{X}$ é martingal, pelo teorema anterior,

$$
\mathbf{E}(X(v))=\mathbf{E}(X(0)) .
$$

Agora, note que $\mathbf{E}(X(0))=\mathbf{E}\left(X^{\tau}(0)\right)$ e portanto de (2.3) e (2.4), ficamos com

$$
\mathbf{E}\left(X^{\tau}(\phi)\right)=\mathbf{E}\left(X^{\tau}(0)\right)
$$

Assim, novamente pelo teorema anterior, resulta que $\mathbf{X}^{\tau}$ é um martingal.

\subsubsection{Martingais limitados em $L^{2}$}

A classe dos martingais contínuos à direita e limitados em $\mathrm{L}^{2}$ terá um papel importante neste trabalho.

Definição 2.39. Denotaremos por $\mathcal{H}^{2}$ a classe de todos os martingais contínuos à direita e limitados em $\mathrm{L}^{2}$.

Proposição 2.40. O espaço $\mathcal{H}^{2}$ munido da a norma $\|M\|_{\mathcal{H}^{2}} \doteq \sup _{t}\left\|M_{t}\right\|_{2}$ é isométricamente isomorfo ao espaço de Hilbert $\mathrm{L}^{2}\left(\Omega, \mathscr{F}_{\infty}, \mathrm{P}\right)$ (e portanto é também um espaço de Hilbert).

Demonstração. Inicialmente, temos pelo teorema da convergência de Doob* que existe $M_{\infty} \in \mathrm{L}^{2}$ tal que $M_{\infty}=\lim _{t \uparrow \infty} M_{t}$, P-q. s. e em L $\mathrm{L}^{2}$. Pela continuidade da norma, temos que ${ }^{\dagger}$

$$
\sup _{t}\left\|M_{t}\right\|_{2}=\lim _{t \rightarrow \infty}\left\|M_{t}\right\|_{2}=\left\|M_{\infty}\right\|_{2}
$$

Segue que $\|M\|_{\mathcal{H}^{2}} \doteq \sup _{t}\left\|M_{t}\right\|_{2}=\left\|M_{\infty}\right\|_{2}$. Assim, identificando martingais que são versões um do outro, temos que $\|\cdot\|_{\mathcal{H}^{2}}$ é um norma em $\mathcal{H}^{2}$ e a aplicação $\mathbf{M} \in \mathcal{H}^{2} \mapsto M_{\infty} \in \mathrm{L}^{2}\left(\Omega, \mathscr{F}_{\infty}, \mathrm{P}\right)$ é uma isometria linear. Finalmente, para mostrar que é um isometria isomórfica basta provar que essa

\footnotetext{
* Veja o teorema 2.35 na página 20. † Veja também as observações após a definiçao 2.25 na página 17.
} 
aplicação também é sobrejetora. Para isto, seja $f \in \mathrm{L}^{2}\left(\Omega, \mathscr{F}_{\infty}, \mathrm{P}\right)$ e defina $M_{t} \doteq \mathbf{E}\left(f \| \mathscr{F}_{t}\right)$, com $t \in \mathbb{R}_{+}$. Para $s \geq t$, temos pelo teorema C.11 que

$$
\begin{aligned}
\mathbf{E}\left(M_{s} \| \mathscr{F}_{t}\right) & =\mathbf{E}\left(\mathbf{E}\left(f \| \mathscr{F}_{s}\right) \| \mathscr{F}_{t}\right) \\
& =\mathbf{E}\left(f \| \mathscr{F}_{t}\right) \\
& =M_{t},
\end{aligned}
$$

Como estamos sob as condições usuais, podemos assumir* que $\left\{M_{t}\right\}_{t \geq 0}$ um martingal contínuo à direita. Agora, pela desigualdade de Jensen ${ }^{\dagger}$,

$$
\left(M_{t}\right)^{2}=\left(\mathbf{E}\left(f \| \mathscr{F}_{t}\right)\right)^{2} \leq \mathbf{E}\left(f^{2} \| \mathscr{F}_{t}\right)
$$

Integrando em $\Omega$ e usando a definição de esperança condicional, ficamos com

$$
\mathbf{E}\left(M_{t}^{2}\right) \leq \int_{\Omega} \mathbf{E}\left(f^{2} \| \mathscr{F}_{t}\right) d \mathrm{P}=\int_{\Omega} f^{2} d \mathrm{P}=\|f\|_{2} .
$$

Tomando o sup para $t$ em $\mathbb{R}_{+}$, resulta

$$
\sup _{t} \mathbf{E}\left(M_{t}^{2}\right)=\lim _{t \rightarrow \infty} \mathbf{E}\left(M_{t}^{2}\right) \doteq\|\mathbf{M}\|_{\mathcal{H}^{2}} \leq\|f\|_{2}<\infty
$$

Portanto $\mathbf{M} \in \mathcal{H}^{2}$ e assim existe $M_{\infty}$ tal que $M_{t}=\mathbf{E}\left(M_{\infty} \| \mathscr{F}_{t}\right)$. Pela definição de $\mathbf{M}$ resulta que $\mathbf{E}\left(M_{\infty} \| \mathscr{F}_{t}\right)=\mathbf{E}\left(f \| \mathscr{F}_{t}\right)$ para todo $t \in \mathbb{R}_{+}$e assim $M_{\infty}=f$. Como queríamos.

Definição 2.41. Definimos H $^{2} \subset \mathcal{H}^{2}$ como espaço dos martingais contínuos em $\mathcal{H}^{2}$ e denotaremos por $c \mathcal{H}_{0}^{2} \subset c \mathcal{H}^{2}$ o espaço de martingais em $c_{\mathcal{H}}^{2}$ que são nulos na origem.

Proposição 2.42. Os espaços $c \mathcal{H}_{0}^{2}$ e $c \mathcal{H}^{2}$ são subespaços fechados de $\mathcal{H}^{2}$.

Demonstração. Seja $\left\{\mathbf{M}_{n}\right\}_{n \geq 1}$ uma sequência de processos em c $\mathcal{H}^{2}$ e $\mathbf{M} \in \mathcal{H}^{2}$ tal que

\footnotetext{
* Veja o teorema 2.29 na página $19 . \quad$ † Veja o teorema C.13 na página 162.
} 
$\left\|\mathbf{M}_{n}-\mathbf{M}\right\|_{\mathcal{H}^{2}} \rightarrow 0$. Pela desigualdade de Doob* temos

$$
\begin{aligned}
\mathbf{E}\left(\left(\sup _{t}\left|M_{n}(t)-M(t)\right|\right)^{2}\right) & \leq 4\left\|M_{n}(\infty)-M(\infty)\right\|_{2}^{2} \\
& \doteq 4\left\|\mathbf{M}_{n}-\mathbf{M}\right\|_{\mathcal{H}^{2}}^{2}
\end{aligned}
$$

Da convergência em $\mathrm{L}^{2}$, resulta que existe uma subsequência tal que

$$
\sup _{t}\left|M_{n_{k}}(t)-M(t)\right| \longrightarrow 0
$$

para quase todo $\omega \in \Omega$. Isto é, a convergência é uniforme em $t$, P-q. s. Como convergência uniforme preserva continuidade, o processo limite $\mathbf{M}$ também é contínuo e provamos o resultado. Finalmente, note que se $\left\{\mathbf{M}_{n}\right\} \subseteq \mathrm{cH}_{0}^{2}$, temos que $M_{n}(0)=0$ para todo $n$ e portanto $M(0)=\lim _{n \rightarrow \infty} M_{n}(0)=0$ e portanto $\mathbf{M} \in \mathrm{cH}_{0}^{2}$.

\subsection{Martingais locais}

Reiteramos aqui que estamos trabalhando no espaço filtrado de probabilidade $\left(\Omega, \mathscr{F},\left\{\mathscr{F}_{t}\right\}_{t \geq 0}, \mathrm{P}\right)$ satisfazendo as condições usuais. Começaremos com uma definição.

Definição 2.43. Dizemos que um tempo de parada $\tau$ reduz um processo estocástico $\mathbf{X}$ quando $\mathbf{X}^{\tau} \mathbb{1}_{[\tau>0]}$ for um martingal adaptado a $\left\{\mathscr{F}_{t \wedge \tau}\right\}_{t \geq 0}$.

Antes de começar a estudar os martingais locais, será útil estabelecer dois resultados.

Proposição 2.44. Sejam $\mathbf{X}$ um processo estocástico adaptado e contínuo à direita e $\tau$ um tempo de parada. Defina $\mathbf{Y} \doteq \mathbf{X}^{\tau}$ ou $\mathbf{Y} \doteq \mathbf{X}^{\tau} \mathbb{1}_{[\tau>0]}$. Então $\mathbf{Y}$ é um martingal com respeito a $\left\{\mathscr{F}_{t \wedge \tau}\right\}_{t \geq 0}$ se, e somente se, $\mathbf{Y}$ for um martingal com respeito a $\left\{\mathscr{F}_{t}\right\}_{t \geq 0}$.

Demonstração. Suponha que $\mathbf{Y}$ seja um martingal com respeito à $\left\{\mathscr{F}_{t}\right\}_{t \geq 0}$. Note que $\left\{\mathscr{F}_{t \wedge \tau}\right\}_{t \geq 0}$ é tal que $\mathscr{F}_{t \wedge \tau} \subseteq \mathscr{F}_{t}$, para todo $t \geq 0$. Afirmamos que $\mathbf{Y}$ martingal com respeito a qualquer outra filtração $\left\{\mathscr{G}_{t}\right\}_{t \geq 0}$ que satisfaça $\mathscr{G}_{t} \subseteq \mathscr{F}_{t}$, para todo $t \geq 0$ e tal que $\mathbf{Y}$ seja adaptado a $\left\{\mathscr{G}_{t}\right\}_{t \geq 0}$. De fato, fixe $0 \leq s<t$ arbitrários. Temos que $\mathbf{E}\left(Y_{t} \| \mathscr{F}_{s}\right)=Y_{s}$ é $\mathscr{G}_{s}$-mensurável e integrável. Então, pela definição de esperança condicional, $\mathbf{E}\left(Y_{t} \| \mathscr{G}_{s}\right)=\mathbf{E}\left(Y_{t} \| \mathscr{F}_{s}\right)=Y_{s}$. Como queríamos.

\footnotetext{
* Veja o teorema 2.27 na página 18.
} 
Reciprocamente, suponha que $\mathbf{Y}$ seja um martingal com respeito a $\left\{\mathscr{F}_{t \wedge \tau}\right\}_{t \geq 0}$. Fixado $0 \leq s \leq t$, sabemos* $^{*}$ que mostrar que $\mathbf{E}\left(Y_{t} \| \mathscr{F}_{s}\right)=Y_{s}$ é equivalente a mostrar que $\mathbf{E}\left(Y_{s} \mathbb{1}_{[A]}\right)=\mathbf{E}\left(Y_{t} \mathbb{1}_{[A]}\right)$ para todo $A \in \mathscr{F}_{s}$. Note que por hipótese, para todo $A \in \mathscr{F}_{s \wedge \tau}$, vale que $\mathbf{E}\left(Y_{s} \mathbb{1}_{[A]}\right)=\mathbf{E}\left(Y_{t} \mathbb{1}_{[A]}\right)$. Agora seja $A \in \mathscr{F}_{s}$ arbitrário. Considere a decomposição $A=(A \cap[\tau \leq s]) \cup(A \cap[\tau>s])$ Como

$$
\mathbf{E}\left(Y_{s} \mathbb{1}_{[A]}\right)=\mathbf{E}\left(Y_{s} \mathbb{1}_{[A \cap[\tau \leq s]]}\right)+\mathbf{E}\left(Y_{s} \mathbb{1}_{[A \cap[\tau>s]]}\right)
$$

$\mathrm{e}$

$$
\mathbf{E}\left(Y_{t} \mathbb{1}_{[A]}\right)=\mathbf{E}\left(Y_{t} \mathbb{1}_{[A \cap[\tau \leq s]]}\right)+\mathbf{E}\left(Y_{t} \mathbb{1}_{[A \cap[\tau>s]]}\right)
$$

basta mostrar que

$$
\mathbf{E}\left(Y_{s} \mathbb{1}_{[A \cap[\tau \leq s]]}\right)=\mathbf{E}\left(Y_{t} \mathbb{1}_{[A \cap[\tau \leq s]]}\right)
$$

e

$$
\mathbf{E}\left(Y_{s} \mathbb{1}_{[A \cap[\tau>s]]}\right)=\mathbf{E}\left(Y_{t} \mathbb{1}_{[A \cap[\tau>s]]}\right) .
$$

Para mostrar (2.5), note que se $\omega \in A \cap[\tau \leq s]$, temos que $X_{t \wedge \tau}=X_{\tau}=X_{s \wedge \tau}$ e portanto $Y_{t}=Y_{s}$, donde segue (2.5). Para mostrar (2.5), basta mostrar que $A \cap[\tau>s] \in \mathscr{F}_{s \wedge \tau}$ e com isso provamos a proposição. Para mostrar que $A \cap[\tau>s] \in \mathscr{F}_{s \wedge \tau}$, temos, pela definição de $\mathscr{F}_{s \wedge \tau}$ que mostrar que para cada $r \geq 0$,

$$
N \doteq A \cap[\tau>s] \cap[s \wedge \tau \leq r] \in \mathscr{F}_{r} .
$$

De fato, temos que $N=A \cap[\tau>s] \cap[s \leq r]$. Caso $s>r$, então $N=\emptyset \in \mathscr{F} r$. Caso $s \leq r$, então $[s \leq r]=\Omega$ e $N=A \cap[s<\tau]$. Agora, seja $h \geq 0$, arbitrário. Temos que $[s<\tau] \cap[s \wedge \tau<h]=[s<\tau] \cap[s<h]$. Mas

$$
[s<\tau] \cap[s<h]=\bigcup_{q \in \mathbb{Q} \cap[0, h]}[s<q<\tau] \in \mathscr{F}_{h}
$$

e portanto $[s<\tau] \in \mathscr{F}_{s \wedge \tau} \subseteq \mathscr{F}_{s}$. Assim, $N=A \cap[s<\tau] \in \mathscr{F}_{s}$ e como $s \leq r$, resulta que $N \in \mathscr{F}_{r}$.

Proposição 2.45. Seja $\tau$ um tempo de parada. Se $\mathbf{X}^{\tau}$ é um martingal então $\tau$ reduz $\mathbf{X}$. Reciprocamente, se $X_{0}$ é integrável e $\tau$ reduz $\mathbf{X}$, então $\mathbf{X}^{\tau}$ é um martingal.

Demonstração. Seja $\mathbf{X}^{\tau}$ é um martingal. Devemos provar que $\mathbf{X}^{\tau} \mathbb{1}_{[\tau>0]}$ também é. De fato, $\mathbf{X}^{\tau} \mathbb{1}_{[\tau>0]}$ é integrável (pois $\mathbf{X}^{\tau}$ é). Agora, fixe $0 \leq s<t$. Como $\mathbb{1}_{[\tau>0]}$ é limitado e $\mathscr{F}_{s}$-mensurável,

\footnotetext{
* Veja o teorema C.8 na página 159.
} 
$\mathbf{E}\left(X_{t}^{\tau} \mathbb{1}_{[\tau>0]} \| \mathscr{F}_{s}\right)=\mathbb{1}_{[\tau>0]} \mathbf{E}\left(X_{t}^{\tau} \| \mathscr{F}_{s}\right)=X_{s}^{\tau} \mathbb{1}_{[\tau>0]}$ como queríamos.

Reciprocamente, temos que $X_{t}^{\tau}=X_{t}^{\tau} \mathbb{1}_{[\tau>0]}+X_{0} \mathbb{1}_{[\tau=0]}$. Como, por hipótese, $\mathbf{X}^{\tau} \mathbb{1}_{[\tau>0]}$ é martingal e $X_{0} \mathbb{1}_{[\tau=0]}$ é integrável, segue que $\mathbf{X}^{\tau}$ é martingal.

Definição 2.46. Seja $\mathbf{X}$ um processo estocástico adaptado e contínuo à direita. Dizemos que $\mathbf{X} e ́$ um martingal local quando existir uma sequência $\left\{\tau_{n}\right\}_{n \geq 1}$ de tempos de parada, tal que

i) $\tau_{n} \uparrow \infty$, P-q.s.

ii) Para cada $n \geq 1, \tau_{n}$ reduz $\mathbf{X}$, isto é, $\mathbf{X}^{\tau_{n}} \mathbb{1}_{\left[\tau_{n}>0\right]}$ é um martingal com respeito a $\left\{\mathscr{F}_{t \wedge \tau}\right\}_{t \geq 0}$.

Nessas condições, dizemos que $\left\{\tau_{n}\right\}_{n \geq 1}$ é uma sequência redutora para $\mathbf{X}$.

Observações: Em vista da proposição 2.44, a condição ii) da definição anterior é equivalente a $\mathbf{X}^{\tau_{n}} \mathbb{1}_{\left[\tau_{n}>0\right]}$ ser um martingal com respeito a $\left\{\mathscr{F}_{t}\right\}_{t \geq 0}$ e em vista da proposição 2.45 , se $X_{0}$ for integrável, então $\tau$ reduz $\mathbf{X}$ se e somente se $\mathbf{X}^{\tau}$ for um martingal. Assim, temos a seguinte definição alternativa de martingal local.

Definição 2.47. Seja $\mathbf{X}$ um processo estocástico adaptado e contínuo à direita tal que $X_{0}$ seja integrável. Dizemos que $\mathbf{X}$ é um martingal local quando existir uma sequência $\left\{\tau_{n}\right\}_{n \geq 1}$ de tempos de parada, tal que

i) $\tau_{n} \uparrow \infty$, P-q.s.

ii) $\mathbf{X}^{\tau_{n}}$ é um martingal com respeito a $\left\{\mathscr{F}_{t}\right\}_{t \geq 0}$ para cada $n \geq 1$.

Observamos que todo martingal é um martingal local. Trataremos da recíproca dessa afirmação adiante.

O próximo resultado será útil na demonstração de algumas propriedades dos martingais locais que vamos apresentar.

Lema 2.48. Sejam $\mathbf{X}$ um processo estocástico adaptado e contínuo à direita e $\sigma \leq \tau$ tempos de parada. Então, se $\tau$ reduz $\mathbf{X}, \sigma$ também reduz $\mathbf{X}$. 
Demonstração. Seja $M_{t} \doteq X_{t \wedge \tau} \mathbb{1}_{[\tau>0]}$. Temos que $\mathbf{M}$ é um martingal e além disso, como $[\sigma>0] \subseteq$ $[\tau>0]$ podemos escrever $\mathbb{1}_{[\sigma>0]}=\mathbb{1}_{[\sigma>0]} \mathbb{1}_{[\tau>0]}$. Agora,

$$
\begin{aligned}
X_{t \wedge \sigma} \mathbb{1}_{[\sigma>0]} & =X_{t \wedge \sigma} \mathbb{1}_{[\tau>0]} \mathbb{1}_{[\sigma>0]} \\
& =X_{t \wedge \sigma}^{\tau} \mathbb{1}_{[\tau>0]} \mathbb{1}_{[\sigma>0]} \\
& =M_{t}^{\sigma} \mathbb{1}_{[\sigma>0]} .
\end{aligned}
$$

Pelo corolário 2.38, $\mathbf{M}^{\sigma}$ é martingal e portanto, como a demonstração da proposição 2.45 , segue que $M_{t}^{\sigma} \mathbb{1}_{[\sigma>0]}$ também é martingal e resulta que $\sigma$ reduz $\mathbf{X}$.

Proposição 2.49. Sejam X um processo estocástico adaptado e contínuo à direita, $\sigma \leq \tau$ tempos de parada e $\xi$ uma variável aleatória $\mathscr{F}_{0}$-mensurável. Então

i) Soma de dois martingais locais é martingal local.

ii) $\xi \mathbf{X}$ é martingal local.

iii) Um martingal local parado é um martingal local.

Demonstração. i) Sejam $\left\{\sigma_{n}\right\}_{n \geq 1}$ e $\left\{\tau_{n}\right\}_{n \geq 1}$ sequências redutoras para $\mathbf{M}$ e $\mathbf{N}$, respectivamente. Agora, defina $\rho_{n}=\sigma_{n} \wedge n$. Temos pela proposição 2.16 que $\rho_{n}$ é um tempo de parada. Obviamente $\left\{\rho_{n}\right\}_{n \geq 1}$ é crescente e $\lim _{n \rightarrow \infty} \rho_{n}=\infty$ q. s. Além disso, $(\mathbf{M}+\mathbf{N})^{\rho_{n}}=\mathbf{M}^{\rho_{n}}+\mathbf{N}^{\rho_{n}}$. Pelo lema anterior, temos que $\rho_{n}$ reduz $\mathbf{M}$ e $\mathbf{N}$, ou seja, $\mathbf{M}^{\rho_{n}} \mathbb{1}_{\left[\rho_{n}>0\right]}$ e $\mathbf{N}^{\rho_{n}} \mathbb{1}_{\left[\rho_{n}>0\right]}$ são martingais e portanto a soma também é*.

ii) Seja $\left\{\tau_{n}\right\}_{n \geq 1}$ uma sequência redutora para $\mathbf{X}$ e defina, para cada $n \geq 1$,

$$
\alpha_{n}= \begin{cases}+\infty & \text { se }|\xi| \leq n \\ 0 & \text { se }|\xi|>n\end{cases}
$$

Note que $\alpha_{n}$ é um tempo de parada. De fato, seja $t \geq 0$ arbitrário. Como $\tau$ é $\mathscr{F}_{0}$-mensurável, resulta que $\left\{\omega \in \Omega: \alpha_{n}(\omega) \leq t\right\}=\{\omega \in \Omega:|\xi(\omega)|>n\} \in \mathscr{F}_{0} \subseteq \mathscr{F}_{t}$. Pela proposição 2.16, $\rho_{n}=\tau_{n} \wedge \alpha_{n}$ é um tempo de parada, e obviamente $\rho_{n} \nearrow \infty$ q. s. Agora,

$$
(\xi \mathbf{X})^{\rho_{n}} \mathbb{1}_{\left[\rho_{n}>0\right]}=\mathbb{1}_{[|\xi| \leq n]} \xi \mathbf{X}^{\rho_{n}} \mathbb{1}_{\left[\rho_{n}>0\right]}
$$

\footnotetext{
* Veja a proposição B.9 na página 156.
} 
Pelo lema anterior, $\mathbf{X}^{\rho_{n}} \mathbb{1}_{\left[\rho_{n}>0\right]}$ é um martingal e como $\mathbb{1}_{[|\xi| \leq n]} \xi$ é uma variável aleatória $\mathscr{F}_{0}$-mensurável e limitada, pelo teorema C.10 na página 160, segue que para $0<s \leq t$,

$$
\begin{aligned}
\mathbf{E}\left((\xi \mathbf{X})^{\rho_{n}} \mathbb{1}_{\left[\rho_{n}>0\right]}(t)|| \mathscr{F}_{s}\right) & =\mathbb{1}_{[|\xi| \leq n]} \xi \mathbf{E}\left(\mathbf{X}^{\rho_{n}} \mathbb{1}_{\left[\rho_{n}>0\right]}(t)|| \mathscr{F}_{s}\right) \\
& =\mathbb{1}_{[|\xi| \leq n]} \xi \mathbf{X}^{\rho_{n}} \mathbb{1}_{[\rho>0]}(s),
\end{aligned}
$$

ou seja, $\left\{\rho_{n}\right\}_{n \geq 1}$ é uma sequência redutora para $\xi \mathbf{X}$.

iii) Seja $\left\{\tau_{n}\right\}_{n \geq 1}$ uma sequência redutora para $\mathbf{X}$. Então

$$
\left(\mathbf{X}^{\tau}\right)^{\tau_{n}} \mathbb{1}_{\left[\tau_{n}>0\right]}=\left(\mathbf{X}^{\tau_{n}} \mathbb{1}_{\left[\tau_{n}>0\right]}\right)^{\tau}
$$

Mas, pelo corolário 2.38, resulta que $\left(\mathbf{X}^{\tau}\right)^{\tau_{n}} \mathbb{1}_{\left[\tau_{n}>0\right]}$ é um martingal.

Proposição 2.50. Seja $\mathbf{X}$ um martingal local. Então existe uma sequência redutora $\left\{\tau_{n}\right\}_{n \geq 1}$ para $\mathbf{X}$ tal que $\tau_{n} \uparrow \infty$ para cada $\omega \in \Omega$ e $\mathbf{X}^{\tau_{n}} \mathbb{1}_{\left[\tau_{n}>0\right]}$ é um martingal uniformemente integrável para cada $n \geq 1$.

Demonstração. Seja $\left\{\rho_{n}\right\}_{n \geq 1}$ uma sequência redutora para $\mathbf{X}$ e seja $N \subseteq \Omega$ um conjunto de medida nula tal que $\rho_{n}(\omega) \uparrow \infty$ para $\omega \in N^{c}$. Defina $\sigma_{n} \doteq n \mathbb{1}_{[N]}+\rho_{n} \mathbb{1}_{\left[N^{c}\right]}$. Temos que $\sigma_{n} \uparrow \infty$ para todo $\omega \in \Omega$ e $\sigma_{n}=\rho_{n}$, P-q.s. Como a filtração $\left\{\mathscr{F}_{t}\right\}$ é aumentada, temos que $\sigma_{n}$ também é tempo de parada. Assim, para $n \geq 1, X_{t}^{\rho_{n}} \mathbb{1}_{\left[\rho_{n}>0\right]}=X_{t}^{\sigma_{n}} \mathbb{1}_{\left[\sigma_{n}>0\right]}$, P-q. s. para cada $t \geq 0$. Segue que $\mathbf{X}^{\sigma_{n}} \mathbb{1}_{\left[\sigma_{n}>0\right]}$ é um martingal, ou seja, $\left\{\sigma_{n}\right\}_{n \geq 1}$ reduz $\mathbf{X}$. Agora, defina $\tau_{n} \doteq \sigma_{n} \wedge n$. Temos que $\tau_{n} \uparrow \infty$ para todo $\omega \in \Omega$. Pelo lema 2.48 temos que $\left\{\tau_{n}\right\}_{n \geq 1}$ é redutora para $\mathbf{X}$. Agora fixe $n \geq 1$ e defina $\mathbf{Y} \doteq \mathbf{X}^{\sigma_{n}} \mathbb{1}_{\left[\sigma_{n}>0\right]}$. Note que $\mathbf{Y}$ é martingal. Como martingal parado também é maringal, $\left\{Y_{t \wedge n}\right\}_{t \geq 0}$ é um martingal e temos que $Y_{t \wedge n}=\mathbf{E}\left(Y_{n} \| \mathscr{F}_{t}\right)$ para todo $t \geq 0$. Pelo teorema de convergência de Doob, segue que $\left\{Y_{t \wedge n}\right\}_{t \geq 0}$ é um martingal uniformemente integrável e portanto observando que

$$
\begin{aligned}
X_{t}^{\tau_{n}} \mathbb{1}_{\left[\tau_{n}>0\right]} & =X_{t \wedge n}^{\sigma_{n}} \mathbb{1}_{\left[\tau_{n}>0\right]} \\
& =X_{t \wedge n}^{\sigma_{n}} \mathbb{1}_{\left[\sigma_{n}>0\right]} \mathbb{1}_{\left[\tau_{n}>0\right]} \\
& =Y_{t \wedge n} \mathbb{1}_{\left[\tau_{n}>0\right]}
\end{aligned}
$$

concluímos que $\left\{X_{t}^{\tau_{n}} \mathbb{1}_{\left[\tau_{n}>0\right]}\right\}_{t \geq 0}$ é martingal uniformemente integrável, como queríamos.

Definição 2.51. Dizemos que um martingal $\mathbf{M}$ é uniformemente limitado quando existir uma con- 
stante positiva $K$ tal que $\left|M_{t}\right| \leq K$, P-q. s. para todo $t \geq 0$.

O próximo resultado mostra que para martingais locais contínuos é possivel selecionar uma sequência redutora de forma que o processo truncado seja um martingal uniformemente limitado.

Proposição 2.52. Sejam $\mathbf{X}$ um martingal local contínuo, $\left\{\rho_{n}\right\}_{n \geq 1}$ uma sequência redutora para $\mathbf{X}$ e defina $\tau_{n} \doteq \inf \left\{t \geq 0:\left|X_{t}\right| \geq n\right\}$. Então $\left\{\sigma_{n}\right\}_{n \geq 1}$, definida para cada $n \geq 1$ por $\sigma_{n} \doteq \rho_{n} \wedge \tau_{n} e^{\prime}$ uma sequência redutora para $\mathbf{X}$ e satisfaz

$$
\left|X^{\sigma_{n}}(t) \mathbb{1}_{\left[\sigma_{n}>0\right]}\right| \leq n
$$

ou seja, $\left\{\mathbf{X}^{\sigma_{n}} \mathbb{1}_{\left[\sigma_{n}>0\right]}\right\}_{t}$ é uniformemente limitado e em particular uniformemente integrável.

Demonstração. Pela proposição $2.15, \tau_{n}$ é um tempo de parada para todo $n \geq 1$. Assim $\left\{\sigma_{n}\right\}_{n \geq 1}$ é uma sequência de tempos de parada tal que $\sigma_{n} \nearrow \infty$, q. s. A continuidade à esquerda de $\mathbf{X}$ garante que $\left|X^{\sigma_{n}}(t)\right| \leq n$ em $\left[\tau_{n}>0\right]$ e portanto em $\left[\sigma_{n}>0\right] \subseteq\left[\tau_{n}>0\right]$. Assim (2.7) vale e o resultado segue pelo lema 2.48 .

Nem todo martingal local é um martingal. Explicitaremos essa afirmação através de um exemplo.

Exemplo 2.53. Seja $(\Omega, \mathscr{F}, \mathrm{P})=\left([0,1], \mathscr{B}_{[0,1]}, \lambda\right)$ onde $\lambda$ é a medida de Lebesgue restrita ao intervalo $[0,1]$ e considere a filtração $\mathscr{F}_{t}=\mathscr{F}$ para todo $t \geq 0$. Considere também $\xi$ uma variável aleatória não integravel. Então $X(t)=\xi$ obviamente não é martingal, mas é um martingal local. De fato,

$$
\tau_{n}= \begin{cases}+\infty & \text { se }|X(0)| \leq n \\ 0 & \text { se }|X(0)|>n\end{cases}
$$

é uma sequência localizadora para $\mathbf{X}$.

O próximo objetivo é achar uma condição necessária e suficiente para que um martingal local seja um martingal.

Definição 2.54. Para cada $a>0$, seja $\mathcal{T}_{a}$ a classe de todos os tempos de parada $\tau: \Omega \rightarrow[0, a]$. Dizemos que um processo adaptado e contínuo à direita é da classe DL (Dirichlet - Doob) se a família de variáveis aleatórias $\left\{X_{\tau}\right\}_{\tau \in \mathcal{T}_{a}}$ for uniformemente integrável para cada a $>0$. 
Proposição 2.55. Um martingal local é um martingal se, e somente se, for da classe DL.

Demonstração. Seja $\mathbf{X}$ um martingal (contínuo à direita) e $\tau \in \mathcal{T}_{a}$ um tempo de parada arbitrário. Como $\tau \leq a$, então pelo teorema 2.36

$$
X(\tau)=\mathbf{E}\left(X(a) \| \mathscr{F}_{\tau}\right),
$$

e pelo lema 2.33, segue que a família $\left\{X(\tau): \tau \in \mathcal{T}_{a}\right\}$ é uniformemente integrável.

Reciprocamente, seja $\mathbf{X}$ um martingal local da classe DL e $\left\{\tau_{n}\right\}_{n \geq 1}$ uma sequência localizadora para $\mathbf{X}$. Para cada $n \geq 1$, defina $Y_{n}(t) \doteq X^{\tau_{n}}(t) \mathbb{1}_{\left[\tau_{n}>0\right]}$. Como para cada $n \geq 1, \mathbf{Y}_{n}$ é um martingal, temos, para $0 \leq s<t$

$$
Y_{n}(s)=\mathbf{E}\left(Y_{n}(t) \| \mathscr{F}_{s}\right) .
$$

Agora, $Y_{n}(t) \stackrel{\text { q.s }}{\longrightarrow} X(t)$ e $Y_{n}(s) \stackrel{\text { q.s }}{\longrightarrow} X(s)$ para quase todo $\omega \in \Omega$. Além disso, $\left\{Y_{n}(t)\right\}_{n}$ e $\left\{Y_{n}(s)\right\}_{n}$ são famílias uniformemente integráveis e portanto* vale também que $Y_{n}(t) \stackrel{\mathrm{L}^{1}}{\rightarrow} X(t)$ e $Y_{n}(s) \stackrel{\mathrm{L}^{1}}{\rightarrow} X(s)$. Resulta pelo teorema C.15 que

$$
X(s)=\lim _{n \rightarrow \infty} Y_{n}(s)=\lim _{n \rightarrow \infty} \mathbf{E}\left(Y_{n}(t) \| \mathscr{F}_{s}\right)=\mathbf{E}\left(X(t) \| \mathscr{F}_{s}\right),
$$

ou seja, $\mathbf{X}$ é um martingal.

Corolário 2.56. Seja $\mathbf{X}$ um martingal local. Se $\mathbf{E}\left(\sup _{t \leq a}\left|X_{t}\right|\right)<\infty$, para todo a $>0$ ou $\mathbf{X}$ for uniformemente limitado, então $\mathbf{X}$ é um martingal.

Demonstração. De fato, como

$$
0 \leq|X(\tau)| \leq \sup _{t \leq a}|X(t)| \quad \forall \tau \in \mathcal{T}_{a}
$$

e como $\sup _{t \leq a}|X(t)|$ é integrável, resulta ${ }^{\dagger}$ que $\mathbf{X} \in \mathrm{DL}$.

Provaremos um resultado agora que será útil no apêndice E.

Proposição 2.57. Seja $\mathbf{X}$ um martingal local não negativo. Então $\mathbf{X}$ é um supermartingal. Além disso, $\mathbf{X}$ é um martingal se, e somente se, tiver esperança constante.

\footnotetext{
* Veja o corolário B.7 na página $155 . \quad$ † Veja a proposição B.9 na página 156, item iv.
} 
Demonstração. Como o processo é não negativo, $\mathbf{E}\left(X_{t}^{-}\right)=0$ e portanto, pela definição 2.25 , basta provar que para $0 \leq s \leq t, \mathbf{E}\left(X_{t}|| \mathscr{F}_{s}\right) \leq X_{s}$. Para isso, considere $\left\{\tau_{n}\right\}_{n \geq 1}$, uma sequência redutora de tempos de parada para $\mathbf{X}$. Temos que $\tau_{n} \uparrow \infty$, P-q. s. e para cada $n \geq 1, \mathbf{X}^{\tau} \mathbb{1}_{[\tau>0]}$ é um martingal. Agora, fixe $n \geq 1$. Temos que

$$
\mathbf{E}\left(X_{t \wedge \tau_{n}} \mathbb{1}_{\left[\tau_{n}>0\right]}|| \mathscr{F}_{s}\right)=X_{s \wedge \tau_{n}} \mathbb{1}_{\left[\tau_{n}>0\right]}
$$

Fazendo $n \uparrow \infty$ e usando o lema de Fatou para esperança condicional* temos que

$$
\begin{aligned}
\mathbf{E}\left(X_{t} \| \mathscr{F}_{s}\right)=\mathbf{E}\left(\liminf _{n} X_{t \wedge \tau_{n}} \mathbb{1}_{\left[\tau_{n}>0\right]} \| \mathscr{F}_{s}\right) & \leq \liminf _{n} \mathbf{E}\left(X_{t \wedge \tau_{n}} \mathbb{1}_{\left[\tau_{n}>0\right]} \| \mathscr{F}_{s}\right) \\
& =\liminf _{n} X_{s \wedge \tau_{n}} \mathbb{1}_{\left[\tau_{n}>0\right]} \\
& =X_{s} .
\end{aligned}
$$

Isso mostra que $\mathbf{X}$ é um supermartingal. Agora, se $\mathbf{X}$ for um martingal, segue que tem média constante. Recipocramente, suponha que $\mathbf{E}\left(X_{t}\right)$ é constante para $t \geq 0$. Pela propriedade supermartingal de $\mathbf{X}$, temos que $\mathbf{E}\left(X_{t} \| \mathscr{F}_{s}\right) \leq X_{s}$, P-q.s. para $0 \leq s \leq t$. Suponha por contradição que $\mathbf{X}$ não fosse um martingal, então para algum $0 \leq s \leq t$ teríamos que $\mathrm{P}\left(\mathbf{E}\left(X_{t} \| \mathscr{F}_{s}\right)<X_{s}\right)>0$. Assim, vamos definir $A \doteq\left\{\omega \in \Omega: \mathbf{E}\left(X_{t} \| \mathscr{F}_{s}\right)<X_{s}\right\}, \quad B \quad \doteq \quad\left\{\omega \in \Omega: \mathbf{E}\left(X_{t} \| \mathscr{F}_{s}\right)=X_{s}\right\} \quad \mathrm{e}$ $C \doteq\left\{\omega \in \Omega: \mathbf{E}\left(X_{t} \| \mathscr{F}_{s}\right)>X_{s}\right\}$ (note que pelo fato de $\mathbf{X}$ ser supermartingal $C$ tem medida nula). Temos que

$$
\begin{aligned}
\mathbf{E}\left(X_{t}\right) & =\mathbf{E}\left(\mathbf{E}\left(X_{t} \| \mathscr{F}_{s}\right)\right) \\
& =\int_{A} \mathbf{E}\left(X_{t} \| \mathscr{F}_{s}\right) d \mathrm{P}+\int_{B} \mathbf{E}\left(X_{t} \| \mathscr{F}_{s}\right) d \mathrm{P}+\int_{C} \mathbf{E}\left(X_{t} \| \mathscr{F}_{s}\right) d \mathrm{P} \\
& <\int_{\Omega} X_{s} d \mathrm{P} \\
& =\mathbf{E}\left(X_{s}\right) .
\end{aligned}
$$

Mas isso é absurdo pois por hipótese $\mathbf{X}$ tinha esperança constante.

\footnotetext{
* Veja o teorema C.16 na página 164.
} 


\title{
Capítulo 3
}

\section{Variação quadrática}

\author{
[Upon proving that the best betting strategy for \\ "Gambler's Ruin" was to bet all on the first trial.] \\ It is true that a man who does this is a fool. I \\ have only proved that a man who does anything \\ else is an even bigger fool. \\ Julian Lowell Coolidge
}

No que se segue, consideraremos que estamos sob as condições usuais*. Seja M um martingal. A menos que $\mathbf{M}$ seja constante, $\mathbf{M}^{2}$ não será um martingal. Um dos objetivos deste capítulo é mostrar que de modo geral $\mathbf{M}^{2}$ difere de um martingal por único processo crescente, contínuo e nulo na origem. Este processo será chamado de variação quadrática de $\mathbf{M}$.

\subsection{Resultados preliminares}

Definição 3.1. Dizemos que um martingal $\mathbf{M}$ é de quadrado integrável quando $\mathbf{E}\left(M^{2}(t)\right)<\infty$, isto é, $M(t) \in \mathrm{L}^{2}$, para todo $t \geq 0$.

Observamos que $\mathbf{M}^{2}$ é um submartingal. De fato, em primeiro lugar, como $\mathbf{M}$ é adaptado e $x \mapsto x^{2}$ é contínua (e portanto, Borel mensurável) segue que $\mathbf{M}^{2}$ também é. Agora, seja $s \leq t$. Temos pela desigualdade de Jensen $^{\dagger}$ que

$$
M^{2}(s)=\left(\mathbf{E}\left(M(t) \| \mathscr{F}_{s}\right)\right)^{2} \leq \mathbf{E}\left(M^{2}(t) \| \mathscr{F}_{s}\right)
$$

\footnotetext{
* Veja a definição 2.24 na página $17 . \quad$ † Veja o teorema C.13 na página 162.
} 
Exemplo 3.2. O movimento Browniano padrão é um martingal de quadrado integrável. De fato,

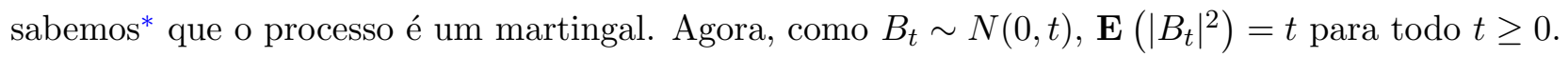

A maioria dos resultados a seguir dependem da próxima proposição que é válida para martingais de quadrado integrável.

Proposição 3.3. Seja $\mathbf{M}$ um martingal de quadrado integrável. Então

$$
\mathbf{E}\left(\left(M_{t}-M_{s}\right)^{2} \| \mathscr{F}_{a}\right)=\mathbf{E}\left(M_{t}^{2}-M_{s}^{2} \| \mathscr{F}_{a}\right), \quad 0 \leq a \leq s \leq t
$$

Demonstração. Temos que

$$
\begin{aligned}
\mathbf{E}\left(M_{t}^{2}-2 M_{t} M_{s}+M_{s}^{2} \| \mathscr{F}_{a}\right) & =\mathbf{E}\left(M_{t}^{2}-M_{s}^{2}-2 M_{t} M_{s}+2 M_{s}^{2} \| \mathscr{F}_{a}\right) \\
& =\mathbf{E}\left(M_{t}^{2}-M_{s}^{2} \| \mathscr{F}_{a}\right)-2 \mathbf{E}\left(M_{t} M_{s}-M_{s}^{2} \| \mathscr{F}_{a}\right) \\
& =\mathbf{E}\left(M_{t}^{2}-M_{s}^{2} \| \mathscr{F}_{a}\right)-2 \mathbf{E}\left(M_{s}\left(M_{t}-M_{s}\right) \| \mathscr{F}_{a}\right) \\
& =\mathbf{E}\left(M_{t}^{2}-M_{s}^{2} \| \mathscr{F}_{a}\right)-2 \mathbf{E}\left(\mathbf{E}\left(M_{s}\left(M_{t}-M_{s}\right) \| \mathscr{F}_{s}\right) \| \mathscr{F}_{a}\right) .
\end{aligned}
$$

Na última igualdade usamos a propriedade da torre ${ }^{\dagger}$ da esperança condicional. Agora, como $M_{s} \in \mathscr{F}_{s}$, $M_{t}-M_{s} \in \mathrm{L}^{1}$ e pela desigualdade de Hölder, $M_{s}\left(M_{t}-M_{s}\right) \in \mathrm{L}^{1}$, estamos sob as hipóteses do teorema C.11 e portanto, como M é martingal,

$$
\mathbf{E}\left(M_{s}\left(M_{t}-M_{s}\right) \| \mathscr{F}_{s}\right)=M_{s} \mathbf{E}\left(M_{t}-M_{s} \| \mathscr{F}_{s}\right)=0 .
$$

Desta última igualdade e de (3.1), provamos o que queríamos.

Lembremos agora do conceito de funções de variação limitada.

Definição 3.4. Seja $\mathcal{P}: 0=t_{0}<t_{1}<\ldots<t_{n}=t$ uma partição do intervalo $[0, t]$. Definimos $|\mathcal{P}|$, o módulo da partição $\mathcal{P}$ como o $\sup _{i}\left|t_{i+1}-t_{i}\right|$. Agora, seja $A: \mathbb{R}_{+} \rightarrow \mathbb{R}$, definimos

$$
S_{t}^{(\mathcal{P}, A)} \doteq \sum_{i}\left|A\left(t_{i+1}\right)-A\left(t_{i}\right)\right|
$$

\footnotetext{
* Veja o exemplo 2.26 na página 18. † Veja o teorema C.11 na página 161.
} 
Dizemos que A é de variação finita quando para cada $t \geq 0$,

$$
S_{t}^{(A)} \doteq \sup _{\mathcal{P}} S_{t}^{(\mathcal{P}, A)}<\infty
$$

Chamamos a função $t \mapsto S_{t}^{(A)}$ de variação total de $A$ em $[0, t]$. Quando $\lim _{t \rightarrow \infty} S_{t}^{(A)}<\infty$, dizemos que $A$ é de variação limitada. Usaremos as notações mais curtas $S_{t}^{(\mathcal{P})}$ e $S_{t}$ quando A estiver implícito no contexto.

Observamos que a variação total é positiva, crescente e nula em $t=0$, funções monótonas são de variação finita (em particular, a função variação total é de variação finita) e qualquer função de variação finita pode ser escrita como a diferença entre duas funções crescentes. Além disso, a função variação total de $A$ é contínua se e somente se $A$ for contínua. As demonstrações destas observações podem ser encontradas em livros de análise real, por exemplo [28]. Na próxima definição, ligamos o conceito de variação finita com processos estocásticos.

Definição 3.5. Dizemos que um processo A é de variação finita quando for adaptado e as trajétorias $t \mapsto A(t, \omega)$ forem finitas, contínuas à direita e de variação finita para quase todo $\omega \in \Omega$.

O próximo teorema mostra que martingais locais contínuos não triviais não são de variação finita. Mais do que apenas uma propriedade interessante desses processos, veremos que este resultado terá consequências profundas no desenvolvimento da teoria.

Teorema 3.6 (Teorema de Fisk). Seja $\mathbf{M}$ um martingal local contínuo de variação finita. Então $\mathbf{M}$ é constante no tempo, isto é, $M_{t}=M_{0}$, P-q.s. para cada $t \geq 0$.

Demonstração. Inicialmente, note que $\left\{M_{t}-M_{0}\right\}_{t \geq 0}$ é um martingal local. De fato, seja $\left\{\tau_{n}\right\}_{n \geq 1}$ uma sequência localizadora para $\mathbf{M}$. Fixe $n \geq 1$, temos que $\mathbf{M}^{\tau_{n}} \mathbb{1}_{\left[\tau_{n}>0\right]}$ é um martingal. Em particular, $M_{0} \mathbb{1}_{\left[\tau_{n}>0\right]}=M_{0}^{\tau_{n}} \mathbb{1}_{\left[\tau_{n}>0\right]} \in \mathrm{L}^{1}$. Portanto $\left\{M_{t}^{\tau_{n}} \mathbb{1}_{\left[\tau_{n}>0\right]}-M_{0} \mathbb{1}_{\left[\tau_{n}>0\right]}\right\}_{t \geq 0}$ é um martingal. Temos que $\left\{\tau_{n}\right\}_{n \geq 1}$ é sequência redutora para $M-M(0)$, o que prova que $\left\{M_{t}-M_{0}\right\}_{t \geq 0}$ é martingal local. Tendo em vista esse fato, podemos, sem perda de generalidade, supor que o martingal local se anula em $t=0$ e assim basta provar que $M_{t}=0$, P-q. s. para cada $t \geq 0$. Seja $S_{t}(\omega)$ a variação total das trajétorias $s \mapsto M_{s}(\omega)$ no intervalo $[0, t]$. Sabemos também que a função crescente $t \mapsto S_{t}(\omega)$ é contínua e de variação finita para todo $\omega \in \Omega$ tal que a trajetória $t \mapsto M_{t}(\omega)$ for contínua e de variação finita. 
Suponha que $\mathbf{M}$ é um martingal tal que exista $K>0$ satisfazendo $\left|S_{t}\right| \leq K$, P-q. s. para todo $t \geq 0$. Isso implica que $\mathbf{M}$ é, para quase todo $\omega \in \Omega$, uniformemente limitada, de fato, $\left|M_{t}\right|=$ $\left|M_{t}-M_{0}\right| \leq S_{t} \leq K$, P-q. s. para todo $t \geq 0$. Agora, fixe $t>0$ e seja $\mathcal{P}: 0=t_{0}<t_{1}<\ldots<t_{n}=t$ uma partição arbitrária do intervalo $[0, t]$. Usando a proposição $3.3\left(\operatorname{com} \mathscr{F}_{a}=\{\emptyset, \Omega\}\right)$ temos

$$
\begin{aligned}
\mathbf{E}\left(M_{t}^{2}\right)=\mathbf{E}\left(M_{t}^{2}-M_{0}^{2}\right) & =\mathbf{E}\left(\sum_{j=1}^{n}\left(M_{t_{j}}^{2}-M_{t_{j-1}}^{2}\right)\right) \\
& =\mathbf{E}\left(\sum_{j=1}^{n}\left(M_{t_{j}}-M_{t_{j-1}}\right)^{2}\right) \\
& \leq \mathbf{E}\left(S_{t} \cdot \sup _{1 \leq j \leq n}\left|M_{t_{j}}-M_{t_{j-1}}\right|\right) .
\end{aligned}
$$

Agora, fazendo $|\mathcal{P}| \rightarrow 0$, temos que $S_{t} \cdot \sup _{1 \leq j \leq n}\left|M_{t_{j}}-M_{t_{j-1}}\right| \longrightarrow 0$ para todo $\omega \in \Omega$ tal que $S_{t}(\omega)<\infty$ e $s \in[0, t] \mapsto M_{t}(\omega)$ é contínuo. Assim, por hipótese, $S_{t} \cdot \sup _{1 \leq j \leq n}\left|M_{t_{j}}-M_{t_{j-1}}\right| \longrightarrow 0$, P-q. s. Além disso, como $\left|S_{t} \cdot \sup _{1 \leq j \leq n}\right| M_{t_{j}}-M_{t_{j-1}}|| \leq 2 K^{2}$, P-q. s., o teorema da convergência dominada garante que

$$
\mathbf{E}\left(S_{t} \cdot \sup _{1 \leq j \leq n}\left|M_{t_{j}}-M_{t_{j-1}}\right|\right) \longrightarrow 0 .
$$

Assim, $\mathbf{E}\left(M_{t}^{2}\right)=0$ e portanto $M_{t}=0$, P-q.s.

De maneira geral, seja $M_{t}$ um martingal local contínuo. Para cada $n \geq 1$, defina $\tau_{n}(\omega) \doteq$ $\inf \left\{t \geq 0: S_{t}(\omega)>n\right\}$ (usamos a convenção de que $\inf \emptyset=\infty$ ). Pela proposição 2.13, para cada $n \geq$ $1, \tau_{n}$ é tempo de parada. Como o processo é de variação finita, $\tau_{n} \nearrow \infty$, P-q. s. Pela proposição 2.49 item iii, $\mathbf{M}^{\tau_{n}}$ é martingal local e $M_{0}^{\tau_{n}}=0$. Assim, seja $S_{t}^{(n)}$ o processo variação total de $\mathbf{M}^{\tau_{n}}$. Temos que $\left|M_{t}^{\tau_{n}}-M_{0}^{\tau_{n}}\right|=\left|M_{t}^{\tau_{n}}\right| \leq\left|S_{t}^{(n)}\right| \leq n$ e assim, vemos que $\mathbf{M}^{\tau_{n}}$ é um martingal local uniformemente limitado e portanto, pelo corolário 2.56, é um martingal. Logo, como no caso anterior, $M_{t}^{\tau_{n}}=0$, P-q. s. para cada $t \geq 0$. Fazendo $n \uparrow \infty$, resulta que $M_{t}=0$, P-q.s. para cada $t \geq 0$. Como queríamos.

Uma importante consequência do teorema de Fisk é o seguinte

Corolário 3.7. Seja $\mathbf{X}$ um processo estocástico. Então existe no máximo um único (módulo indistinguibilidade) processo contínuo de variação finita $\mathbf{A}$ tal que $A(0)=0$ e $\left\{X_{t}-A_{t}\right\}_{t \geq 0}$ é um martingal 
local.

Demonstração. Suponha que existissem A e B processos de variação finita tais que $A(0)=B(0)=0$ e $\mathbf{X}-\mathbf{A}=\mathbf{M}$ e $\mathbf{X}-\mathbf{B}=\mathbf{N}$ com $\mathbf{M}$ e $\mathbf{N}$ martingais locais. Então, $\mathbf{B}-\mathbf{A}=\mathbf{M}-\mathbf{N}$. Como $\mathbf{M}-\mathbf{N}$ é martingal local, e $\mathbf{B}-\mathbf{A}$ é de variação finita, temos que $\mathbf{B}-\mathbf{A}$ é um martingal local contínuo de variação finita e portanto, pelo teorema anterior, $B(t)-A(t)=B(0)-A(0)=0$, P-q. s. para cada $t \geq 0$ e portanto $\mathbf{A}$ e $\mathbf{B}$ são indistinguíveis*.

Definição 3.8. Sejam $\mathbf{X}$ um processo estocástico, $0 \leq a<b$ e $\mathcal{P}: a=t_{0}<t_{1}<\ldots<t_{n}=b$ uma partição de $[a, b]$. Definimos a variável aleatória $Q^{(\mathcal{P}, \mathbf{X})}$ por

$$
Q^{(\mathcal{P}, \mathbf{X})}(\omega) \doteq \sum_{j=1}^{n}\left(X\left(t_{j}, \omega\right)-X\left(t_{j-1}, \omega\right)\right)^{2}
$$

De maneira geral, se $\mathcal{P}: 0=t_{0}<t_{1}<\ldots<t_{n}<\ldots$ é uma partição de $[0, \infty)$ com número finito de pontos em cada intervalo finito, definimos o processo $\mathbf{Q}^{(\mathcal{P}, \mathbf{X})}$ como

$$
Q^{(\mathcal{P}, \mathbf{X})}(t, \omega) \doteq \sum_{j=1}^{k(t)}\left(X\left(t_{j}, \omega\right)-X\left(t_{j-1}, \omega\right)\right)^{2}+\left(X(t, \omega)-X\left(t_{k}, \omega\right)\right)^{2}
$$

onde $k(t)=\max \left\{k \geq 0: t_{k} \leq t\right\}$. Se a partição $\mathcal{P}$ contém o ponto $t$, então podemos escrever

$$
Q^{(\mathcal{P}, \mathbf{X})}(t, \omega) \doteq \sum_{j=1}^{n}\left(X\left(t_{j}, \omega\right)-X\left(t_{j-1}, \omega\right)\right)^{2}
$$

onde $n$ é tal que $t_{n}=t$. Em particular, se $\mathcal{P}$ é uma partição de $[0, t]$, podemos pensar em $\mathcal{P}$ como uma partição de $[0, \infty)$ que contém $t$. Nesse caso, $Q^{(\mathcal{P}, \mathbf{X})}=Q^{(\mathcal{P}, \mathbf{X})}(t)$.

Lema 3.9. Sejam $\mathbf{M}$ um martingal contínuo e uniformemente limitado e $\mathcal{P}: 0=t_{0}<t_{1}<\ldots<$ $t_{n}<\ldots$ uma partição de $[0, \infty)$ com número finito de pontos em cada intervalo finito. Então o processo $\mathbf{M}^{2}-\mathbf{Q}^{(\mathcal{P}, \mathbf{M})}$ é um martingal contínuo.

Demonstração. A continuidade do processo segue imediatamente da continuidade de M. Da limitação uniforme de $\mathbf{M}$, segue que para cada $t \geq 0, M_{t}^{2}-Q_{t}^{(\mathcal{P}, \mathbf{M})} \in \mathrm{L}^{1}$. Resta provar que o processo satisfaz

\footnotetext{
* Veja a proposição 2.3 na página 6.
} 
a equação de martingal, isto é, para $0 \leq s<t, \mathbf{E}\left(M_{t}^{2}-Q_{t}^{(\mathcal{P}, \mathbf{M})} \| \mathscr{F}_{s}\right)=M_{s}^{2}-Q_{s}^{(\mathcal{P}, \mathbf{M})}$ ou, equivalentemente, $\mathbf{E}\left(M_{t}^{2}-M_{s}^{2} \| \mathscr{F}_{s}\right)=\mathbf{E}\left(Q_{t}^{(\mathcal{P}, \mathbf{M})}-Q_{s}^{(\mathcal{P}, \mathbf{M})} \| \mathscr{F}_{s}\right)$. De fato, sejam $0 \leq s<t$ arbitrário e defina $m=\max \left\{k: t_{k} \leq s\right\}$ e $n=\max \left\{k: t_{k} \leq t\right\}$. Temos que $m \leq n$ e

$$
Q_{t}^{(\mathcal{P}, \mathbf{M})}-Q_{s}^{(\mathcal{P}, \mathbf{M})}=-\left(M_{s}-M_{t_{m}}\right)^{2}+\sum_{j=m+1}^{n}\left(M_{t_{j}}-M_{t_{j-1}}\right)^{2}+\left(M_{t}-M_{t_{n}}\right)^{2} .
$$

O resultado segue desta última relação e da proposição 3.3, pois

$$
\begin{aligned}
\mathbf{E}\left(Q_{t}^{(\mathcal{P}, \mathbf{M})}-Q_{s}^{(\mathcal{P}, \mathbf{M})} \| \mathscr{F}_{s}\right) & =\mathbf{E}\left(\left(M_{t_{m}}^{2}-M_{s}^{2}\right)+\sum_{j=m+1}^{n}\left(M_{t_{j}}^{2}-M_{t_{j-1}}^{2}\right)+\left(M_{t}^{2}-M_{s}^{2}\right) \| \mathscr{F}_{s}\right) \\
& =\mathbf{E}\left(M_{t}^{2}-M_{t_{n}}^{2} \| \mathscr{F}_{s}\right) .
\end{aligned}
$$

\subsection{Variação quadrática para martingais contínuos uniformemente limitados}

O próximo resultado é uma das pedras fundamentais para o desenvolvimento da teoria do cálculo estocástico. A demonstração é originalmente de H. Kunita e a versão que apresentamos aqui é a mesma encontrada em [6,16, 29].

Teorema 3.10. Seja $\mathbf{M}$ um martingal q.s. contínuo e uniformemente limitado. Então existe um único processo contínuo de variação finita $\mathbf{A}$ tal que $A(0)=0$ e $\mathbf{M}^{2}-\mathbf{A}$ é um martingal. Chamamos

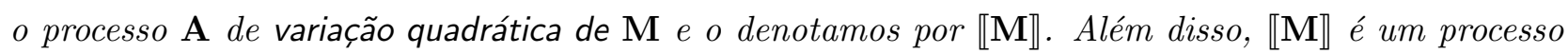
adaptado, crescente, $\llbracket \mathbf{M} \rrbracket(t) \in \mathrm{L}^{2} e$

$$
\llbracket \mathbf{M} \rrbracket(t)=\lim _{|\mathcal{P}| \rightarrow 0} Q^{(\mathcal{P}, \mathbf{M})} \quad \text { em } \mathrm{L}^{2}, \text { para cada } t \geq 0
$$

onde o limite é tomado sob todas as partições $\mathcal{P}$ do intervalo $[0, t]$.

Demonstração. A unicidade é consequência imediata do corolário 3.7. Observamos que basta provar a existência do processo desejado em cada intervalo finito $[0, r]$ pois pela unicidade em cada um desses intervalos, podemos estender o processo para um definido em $[0, \infty)$. Agora, se $\mathbf{M}^{2}-\mathbf{A}$ for um martingal (e portanto adaptado) a adaptabilidade de $\mathbf{A}$ resulta da identidade $A_{t}=M_{t}^{2}-\left(M_{t}^{2}-A_{t}\right)$. 
Vamos agora tratar da existência. Seja $K<\infty$ a constante que limita $\mathbf{M}$, isto é, $\left|M_{t}\right| \leq K$, P-q.s., para todo $t \geq 0$. Inicialmente, vamos provar que para $r>0$, existe o limite em $\mathrm{L}^{2} \tilde{A}_{r}=$ $\lim _{|\mathcal{P}| \rightarrow 0} Q_{r}^{(\mathcal{P}, \mathbf{M})}$. Feito isso, vamos escolher um representante da classe de equivalência que satisfaz as propriedades do teorema.

Fixe $r>0$ e sejam $\mathcal{P}_{1}$ e $\mathcal{P}_{2}$ partições de $[0, r]$. Pela completude de $\mathrm{L}^{2}$, basta mostrar que para $\left|\mathcal{P}_{1}\right|+\mid \mathcal{P}_{2} \rightarrow 0$, vale

$$
\left\|Q^{\left(\mathcal{P}_{1}, \mathbf{M}\right)}-Q^{\left(\mathcal{P}_{2}, \mathbf{M}\right)}\right\|_{2} \longrightarrow 0
$$

Para isso, fixe $\mathcal{P}_{1}$ e $\mathcal{P}_{2}$, partições de $[0, r]$ e defina $\mathcal{P}: 0=s_{0}<s_{1}<\ldots<s_{m}=r$, o refinamento comum dessas duas partições. Pelo lema anterior, $\mathbf{M}^{2}-\mathbf{Q}^{\left(\mathcal{P}_{1}, \mathbf{M}\right)}$ e $\mathbf{M}^{2}-\mathbf{Q}^{\left(\mathcal{P}_{2}, \mathbf{M}\right)}$, são martingais e portanto, a diferença entre esses dois processos

$$
X_{t} \doteq Q_{t}^{\left(\mathcal{P}_{1}, \mathbf{M}\right)}-Q_{t}^{\left(\mathcal{P}_{2}, \mathbf{M}\right)}
$$

é um martingal contínuo e uniformemente limitado. Novamente pelo lema anterior, temos que $\mathbf{X}^{2}-$ $\mathbf{Q}^{(\mathcal{P}, \mathbf{X})}$ é um martingal e portanto tem média constante. Como $X_{0}=0$, resulta que essa constante é zero e assim

$$
\mathbf{E}\left(X_{r}^{2}-Q_{r}^{(\mathcal{P}, \mathbf{X})}\right)=0 \Longrightarrow \mathbf{E}\left(X_{r}^{2}\right)=\mathbf{E}\left(Q_{r}^{(\mathcal{P}, \mathbf{X})}\right)
$$

e, portanto,

$$
\left\|Q_{r}^{\left(\mathcal{P}_{1}, \mathbf{M}\right)}-Q_{r}^{\left(\mathcal{P}_{2}, \mathbf{M}\right)}\right\|_{2}=\mathbf{E}\left(X_{r}^{2}\right)=\mathbf{E}\left(Q_{r}^{(\mathcal{P}, \mathbf{X})}\right) .
$$

Agora, defina $Y_{t} \doteq Q_{t}^{\left(\mathcal{P}_{1}, \mathbf{M}\right)}$ e $Z_{t} \doteq Q_{t}^{\left(\mathcal{P}_{2}, \mathbf{M}\right)}$. Usando a desigualdade $(a-b)^{2} \leq 2\left(a^{2}+b^{2}\right)$, ficamos com

$$
\begin{aligned}
Q_{r}^{(\mathcal{P}, \mathbf{X})} & =\sum_{j=1}^{m}\left(X_{s_{j}}-X_{s_{j-1}}\right)^{2} \\
& =\sum_{j=1}^{m}\left(\left(Y_{s_{j}}-Y_{s_{j-1}}\right)+\left(Z_{s_{j}}-Z_{s_{j-1}}\right)\right)^{2} \\
& \leq 2\left[\sum_{j=1}^{m}\left(Y_{s_{j}}-Y_{s_{j-1}}\right)^{2}+\left(Z_{s_{j}}-Z_{s_{j-1}}\right)^{2}\right] \\
& =2\left(Q_{r}^{(\mathcal{P}, \mathbf{Y})}+Q_{r}^{(\mathcal{P}, \mathbf{Z})}\right) .
\end{aligned}
$$


Tendo em vista essa última estimativa e (3.3), temos que para provar (3.3) basta mostrar que para $\left|\mathcal{P}_{1}\right|+\left|\mathcal{P}_{2}\right| \rightarrow 0$

$$
\mathbf{E}\left(Q_{r}^{(\mathcal{P}, \mathbf{Y})}\right) \longrightarrow 0
$$

Para isto, vamos denotar os pontos da partição $\mathcal{P}_{1}$ por $t_{j}$ e $t_{n}=r$. Sejam $k \leq m$ e $j$ tal que $t_{j} \leq s_{k-1}<s_{k} \leq t_{j+1}$ (essa escolha é sempre possível pois $\mathcal{P}$ refina $\mathcal{P}_{1}$ ). Pela equação (3.2) da definição 3.8 , temos que

$$
\begin{aligned}
Y_{s_{k}}-Y_{s_{k-1}} & =Q_{s_{k}}^{\left(\mathcal{P}_{1}, \mathbf{M}\right)}-Q_{s_{k-1}}^{\left(\mathcal{P}_{1}, \mathbf{M}\right)} \\
& =\left(M_{s_{k}}-M_{t_{j}}\right)^{2}-\left(M_{s_{k-1}}-M_{t_{j}}\right)^{2} \\
& =\left(M_{s_{k}}-M_{s_{k-1}}\right)\left(M_{s_{k}}+M_{s_{k-1}}-2 M_{t_{j}}\right) .
\end{aligned}
$$

Defina agora

$$
C_{\delta}(\omega) \doteq \sup \{M(s, \omega)+M(q, \omega)-2 M(t, \omega): t \leq q<s, s-t<\delta\}
$$

Pela continuidade de $\mathbf{M}$, esse supremo pode ser calculado tomando $t, q, s \in \mathbb{Q}$ e portanto $C_{\delta}$ é mensurável, ou seja, uma variável aleatória não negativa. Além disso, como $\left|M_{t}\right| \leq K$, para todo $t \geq 0,\left|C_{\delta}\right| \leq 16 K^{2}$. Como $\mathbf{M}$ é q. s. contínuo, as trajétorias definidas no compacto $[0, r], t \mapsto M(t, \omega)$ são q. s. uniformemente contínuas e assim concluímos que $C(\delta) \rightarrow 0$, P-q. s. quando $\delta \downarrow 0$. De (3.6), temos que

$$
\left(Y_{s_{k}}-Y_{s_{k-1}}\right)^{2} \leq C_{\left|\mathcal{P}_{1}\right|}\left(M_{s_{k}}-M_{s_{k-1}}\right)^{2}
$$

Somando sobre todos os pontos da partição $\mathcal{P}$, ficamos com

$$
Q_{r}^{(\mathcal{P}, \mathbf{Y})} \leq C_{\left|\mathcal{P}_{1}\right|} Q_{r}^{(\mathcal{P}, \mathbf{M})}
$$

e assim

$$
\mathbf{E}\left(Q_{r}^{(\mathcal{P}, \mathbf{Y})}\right) \leq \mathbf{E}\left(C_{\left|\mathcal{P}_{1}\right|} Q_{r}^{(\mathcal{P}, \mathbf{M})}\right) .
$$

Agora, pela desigualdade de Cauchy-Schwartz,

$$
\mathbf{E}\left(Q_{r}^{(\mathcal{P}, \mathbf{Y})}\right) \leq \sqrt{\mathbf{E}\left(C_{\left|\mathcal{P}_{1}\right|}^{2}\right)} \sqrt{\mathbf{E}\left(\left(Q_{r}^{(\mathcal{P}, \mathbf{M})}\right)^{2}\right)} .
$$




\subsection{VARIAÇÃO QUADRÁTICA PARA MARTINGAIS CONTÍNUOS UNIFORMEMENTE LIMITADOS41}

Agora, pelo teorema da convergência dominada, $\mathbf{E}\left(C_{\left|\mathcal{P}_{1}\right|}^{2}\right) \rightarrow 0$, então, para mostrar (3.5) basta mostrar que $\mathbf{E}\left(\left(Q_{r}^{(\mathcal{P}, \mathbf{M})}\right)^{2}\right)$ é limitado por uma constante independente de $\mathcal{P}_{1}$ e $\mathcal{P}$. Para isso, começamos observando que

$$
\sum_{j=k+1}^{m}\left(M_{s_{j}}-M_{s_{j-1}}\right)^{2}=Q_{r}^{(\mathcal{P}, \mathbf{M})}-Q_{s_{k}}^{(\mathcal{P}, \mathbf{M})}
$$

$\mathrm{e}$

$$
\left(M_{s_{k}}-M_{s_{k-1}}\right)^{2}=Q_{s_{k}}^{(\mathcal{P}, \mathbf{M})}-Q_{s_{k-1}}^{(\mathcal{P}, \mathbf{M})}
$$

assim,

$$
\begin{aligned}
\sum_{1 \leq k<j \leq m}\left(M_{s_{k}}-M_{s_{k-1}}\right)^{2}\left(M_{s_{j}}-M_{s_{j-1}}\right)^{2} & =\sum_{k=1}^{m}\left(M_{s_{k}}-M_{s_{k-1}}\right)^{2} \sum_{j=k+1}^{m}\left(M_{s_{j}}-M_{s_{j-1}}\right)^{2} \\
& =\sum_{k=1}^{m}\left(M_{s_{k}}-M_{s_{k-1}}\right)^{2}\left(Q_{r}^{(\mathcal{P}, \mathbf{M})}-Q_{\left.s_{k}, \mathbf{M}\right)}^{(\mathcal{P}}\right) \\
& =\sum_{k=1}^{m}\left(Q_{\left.s_{k}, \mathbf{M}\right)}^{(\mathcal{P})} Q_{s_{k-1}}^{(\mathcal{P}, \mathbf{M})}\right)\left(Q_{r}^{(\mathcal{P}, \mathbf{M})}-Q_{\left.s_{k}, \mathbf{M}\right)}^{(\mathcal{P})} .\right.
\end{aligned}
$$

Assim de (3.7),

$$
\left(Q_{r}^{(\mathcal{P}, \mathbf{M})}\right)^{2}=2 \sum_{k=1}^{m}\left(Q_{s_{k}}^{(\mathcal{P}, \mathbf{M})}-Q_{s_{k-1}}^{(\mathcal{P}, \mathbf{M})}\right)\left(Q_{r}^{(\mathcal{P}, \mathbf{M})}-Q_{s_{k}}^{(\mathcal{P}, \mathbf{M})}\right)+\sum_{k=1}^{m}\left(M_{s_{k}}-M_{s_{k-1}}\right)^{4}
$$

Agora, como $\mathbf{M}^{2}-\mathbf{Q}^{(\mathcal{P}, \mathbf{M})}$ é um martingal e $0 \leq s_{k} \leq r$, temos $\mathbf{E}\left(M_{r}^{2}-Q_{r}^{(\mathcal{P}, \mathbf{M})} \| \mathscr{F}_{s_{k}}\right)=M_{s_{k}}^{2}-$ $Q_{s_{k}}^{(\mathcal{P}, \mathbf{M})}$ ou, equivalentemente, $\mathbf{E}\left(M_{r}^{2}-M_{s_{k}}^{2} \| \mathscr{F}_{s_{k}}\right)=\mathbf{E}\left(Q_{r}^{(\mathcal{P}, \mathbf{M})}-Q_{s_{k}}^{(\mathcal{P}, \mathbf{M})} \| \mathscr{F}_{s_{k}}\right)$. Pela proposição 3.3 temos portanto

$$
\mathbf{E}\left(Q_{r}^{(\mathcal{P}, \mathbf{M})}-Q_{s_{k}}^{(\mathcal{P}, \mathbf{M})} \| \mathscr{F}_{s_{k}}\right)=\mathbf{E}\left(M_{r}^{2}-M_{s_{k}}^{2} \| \mathscr{F}_{s_{k}}\right)=\mathbf{E}\left(\left(M_{r}-M_{s_{k}}\right)^{2} \| \mathscr{F}_{s_{k}}\right) .
$$

Agora, como $Q_{s_{k}}^{(\mathcal{P}, \mathbf{M})}-Q_{s_{k-1}}^{(\mathcal{P}, \mathbf{M})}$ é limitado e $\mathscr{F}_{s_{k}}$-mensurável, temos pelo teorema C.10 na página 160 e por (3.9) que

$$
\mathbf{E}\left(\left(Q_{s_{k}}^{(\mathcal{P}, \mathbf{M})}-Q_{s_{k-1}}^{(\mathcal{P}, \mathbf{M})}\right)\left(Q_{r}^{(\mathcal{P}, \mathbf{M})}-Q_{s_{k}}^{(\mathcal{P}, \mathbf{M})}\right) \| \mathscr{F}_{s_{k}}\right)=\mathbf{E}\left(\left(Q_{s_{k}}^{(\mathcal{P}, \mathbf{M})}-Q_{s_{k-1}}^{(\mathcal{P}, \mathbf{M})}\right)\left(M_{r}-M_{s_{k}}\right)^{2} \| \mathscr{F}_{s_{k}}\right) .
$$


Usando a proposição $3.3\left(\operatorname{com} \mathscr{F}_{a}=\{\emptyset, \Omega\}\right)$, o fato de $\left(M_{t}-M_{s}\right)^{2} \leq 4 K^{2}$ e a positividade de $\left(Q_{s_{k}}^{(\mathcal{P}, \mathbf{M})}-Q_{s_{k-1}}^{(\mathcal{P}, \mathbf{M})}\right)$, ficamos com

$$
\begin{aligned}
\mathbf{E}\left(\left(Q_{s_{k}}^{(\mathcal{P}, \mathbf{M})}-Q_{s_{k-1}}^{(\mathcal{P}, \mathbf{M})}\right)\left(Q_{r}^{(\mathcal{P}, \mathbf{M})}-Q_{s_{k}}^{(\mathcal{P}, \mathbf{M})}\right)\right) & =\mathbf{E}\left(\left(Q_{s_{k}}^{(\mathcal{P}, \mathbf{M})}-Q_{s_{k-1}}^{(\mathcal{P}, \mathbf{M})}\right)\left(M_{r}-M_{s_{k}}\right)^{2}\right) \\
& \leq \mathbf{E}\left(4 K^{2}\left(Q_{s_{k}}^{(\mathcal{P}, \mathbf{M})}-Q_{s_{k-1}}^{(\mathcal{P}, \mathbf{M})}\right)\right) .
\end{aligned}
$$

Tomando o valor esperado em (3.8) temos, por esta última desigualdade que

$$
\begin{aligned}
\mathbf{E}\left(\left(Q_{r}^{(\mathcal{P}, \mathbf{M})}\right)^{2}\right) & =\mathbf{E}\left(2 \sum_{k=1}^{m}\left(Q_{s_{k}}^{(\mathcal{P}, \mathbf{M})}-Q_{s_{k-1}}^{(\mathcal{P}, \mathbf{M})}\right)\left(Q_{r}^{(\mathcal{P}, \mathbf{M})}-Q_{s_{k}}^{(\mathcal{P}, \mathbf{M})}\right)+\sum_{k=1}^{m}\left(M_{s_{k}}-M_{s_{k-1}}\right)^{4}\right) \\
& =\sum_{k=1}^{m} \mathbf{E}\left(2\left(Q_{s_{k}}^{(\mathcal{P}, \mathbf{M})}-Q_{s_{k-1}}^{(\mathcal{P}, \mathbf{M})}\right)\left(Q_{r}^{(\mathcal{P}, \mathbf{M})}-Q_{s_{k}}^{(\mathcal{P}, \mathbf{M})}\right)+\left(M_{s_{k}}-M_{s_{k-1}}\right)^{4}\right) \\
& \leq \sum_{k=1}^{m} \mathbf{E}\left(8 K^{2}\left(Q_{s_{k}}^{(\mathcal{P}, \mathbf{M})}-Q_{s_{k-1}}^{(\mathcal{P}, \mathbf{M})}\right)+\left(M_{s_{k}}-M_{s_{k-1}}\right)^{4}\right) .
\end{aligned}
$$

Como $\sum_{k=1}^{m}\left(Q_{s_{k}}^{(\mathcal{P}, \mathbf{M})}-Q_{s_{k-1}}^{(\mathcal{P}, \mathbf{M})}\right)=Q_{r}^{(\mathcal{P}, \mathbf{M})}-Q_{0}^{(\mathcal{P}, \mathbf{M})}=Q_{r}^{(\mathcal{P}, \mathbf{M})}$, ficamos com

$\mathbf{E}\left(\left(Q_{r}^{(\mathcal{P}, \mathbf{M})}\right)^{2}\right) \leq \mathbf{E}\left(8 K^{2} Q_{r}^{(\mathcal{P}, \mathbf{M})}+\sum_{k=1}^{m}\left(M_{s_{k}}-M_{s_{k-1}}\right)^{4}\right)$

Usando que $\left(M_{s_{k}}-M_{s_{k-1}}\right)^{4} \leq 4 K^{2}\left(M_{s_{k}}-M_{s_{k-1}}\right)^{2}$, temos que

$$
\begin{aligned}
\mathbf{E}\left(\left(Q_{r}^{(\mathcal{P}, \mathbf{M})}\right)^{2}\right) & \leq \mathbf{E}\left(8 K^{2} Q_{r}^{(\mathcal{P}, \mathbf{M})}+4 K^{2} Q_{r}^{(\mathcal{P}, \mathbf{M})}\right) \\
& =12 K^{2} \mathbf{E}\left(Q_{r}^{(\mathcal{P}, \mathbf{M})}\right)
\end{aligned}
$$

Agora, como $\mathbf{M}^{2}-\mathbf{Q}^{(\mathcal{P}, \mathbf{M})}$ é martingal, tem média constante e assim,

$$
\mathbf{E}\left(M_{r}^{2}-Q_{r}^{(\mathcal{P}, \mathbf{M})}\right)=\mathbf{E}\left(M_{0}^{2}-Q_{0}^{(\mathcal{P}, \mathbf{M})}\right)=\mathbf{E}\left(M_{0}^{2}\right)
$$


Portanto

$$
\mathbf{E}\left(Q_{r}^{(\mathcal{P}, \mathbf{M})}\right)=\mathbf{E}\left(M_{r}^{2}-M_{0}^{2}\right) \leq \mathbf{E}\left(M_{r}^{2}\right) \leq K^{2}
$$

De (3.10) e (3.11), resulta que

$$
\mathbf{E}\left(\left(Q_{r}^{(\mathcal{P}, \mathbf{M})}\right)^{2}\right) \leq 12 K^{4}
$$

e temos que (3.5) vale.

Agora que provamos a existência do limite $\tilde{A}_{t}=\lim _{|\mathcal{P}| \rightarrow 0} Q_{t}^{(\mathcal{P}, \mathbf{M})}$ em L ${ }^{2}$, vamos mostrar que as variáveis aleatórias $A_{t}$ podem ser escolhidas da classe de equivalência $\tilde{A}_{t}$ de tal forma que o processo estocástico resultante $\mathbf{A}$ tenha as propriedades desejadas. Vamos começar provando que podemos selecionar um representante com trajétorias q.s. contínuas. Para isto, fixe $r>0$ e seja $\left\{\mathcal{P}_{n}\right\}_{n \geq 1}$ uma sequência de partições do intervalo $[0, r]$ tal que $\mathcal{P}_{n} \subseteq \mathcal{P}_{n+1}$ e $\cup_{n} \mathcal{P}_{n}$ é denso em $[0, r]$. Sob essas condições, temos que $\lim _{n \rightarrow \infty}\left|\mathcal{P}_{n}\right|=0$ e pelo o que acabamos de mostrar, a sequência $\left\{Q^{\left(\mathcal{P}_{n}, \mathbf{M}\right)}(r)_{n}\right\}_{n \geq 1}$ converge em $\mathrm{L}^{2}$. Fixe $n \geq 1$. Como o espaço de probabilidade é finito, $\|f\|_{1} \leq$ $\|f\|_{2}$, então

$$
\begin{aligned}
\mathbf{E}\left(\sum_{n} \sup _{t \leq r}\left|Q_{t}^{\left(\mathcal{P}_{n+1}, \mathbf{M}\right)}-Q_{t}^{\left(\mathcal{P}_{n}, \mathbf{M}\right)}\right|\right) & =\sum_{n} \mathbf{E}\left(\sup _{t \leq r}\left|Q_{t}^{\left(\mathcal{P}_{n+1}, \mathbf{M}\right)}-Q_{t}^{\left(\mathcal{P}_{n}, \mathbf{M}\right)}\right|\right) \\
& =\sum_{n}\left\|\sup _{t \leq r}\left|Q_{t}^{\left(\mathcal{P}_{n+1}, \mathbf{M}\right)}-Q_{t}^{\left(\mathcal{P}_{n}, \mathbf{M}\right)}\right|\right\| \|_{1} \\
& \leq \sum_{n}\left\|\sup _{t \leq r}\left|Q_{t}^{\left(\mathcal{P}_{n+1}, \mathbf{M}\right)}-Q_{t}^{\left(\mathcal{P}_{n}, \mathbf{M}\right)}\right|\right\| \|_{2}
\end{aligned}
$$

Pela desigualdade de Doob*, temos que

$$
\sum_{n}\left\|\sup _{t \leq r}\left|Q_{t}^{\left(\mathcal{P}_{n+1}, \mathbf{M}\right)}-Q_{t}^{\left(\mathcal{P}_{n}, \mathbf{M}\right)}\right|\right\|_{2} \leq 2 \sum_{n}\left\|Q_{r}^{\left(\mathcal{P}_{n+1}, \mathbf{M}\right)}-Q_{r}^{\left(\mathcal{P}_{n}, \mathbf{M}\right)}\right\|_{2}
$$

É fácil ver que trocando, se necessário, $\left\{\mathcal{P}_{n}\right\}_{n \geq 1}$ por uma subsequência apropriada, podemos assumir que

$$
\sum_{n}\left\|Q_{r}^{\left(\mathcal{P}_{n+1}, \mathbf{M}\right)}-Q_{r}^{\left(\mathcal{P}_{n}, \mathbf{M}\right)}\right\|_{2}<\infty
$$

\footnotetext{
* Veja o teorema 2.27 na página 18.
} 
dessa forma, por (3.13) e (3.14) temos de (3.12) que

$$
\sum_{n} \sup _{t \leq r}\left|Q_{t}^{\left(\mathcal{P}_{n+1}, \mathbf{M}\right)}-Q_{t}^{\left(\mathcal{P}_{n}, \mathbf{M}\right)}\right|<\infty, \quad \mathrm{P}-\mathrm{q} . \mathrm{s} .
$$

Agora, seja $\omega \in \Omega$ no conjunto onde a soma acima é finita, então $\lim _{n \rightarrow \infty} \sup _{t \leq r}\left|Q_{t}^{\left(\mathcal{P}_{n+1}, \mathbf{M}\right)}-Q_{t}^{\left(\mathcal{P}_{n}, \mathbf{M}\right)}\right|=$ 0 . Isso mostra que que para $n \uparrow \infty$ as trajétorias contínuas $t \in[0, r] \mapsto Q_{t}^{\left(\mathcal{P}_{n+1}, \mathbf{M}\right)}$ convergem uniformemente para alguma a trajétoria contínua $t \in[0, r] \mapsto A(t, \omega)$. Em particular, $Q_{t}^{\left(\mathcal{P}_{n}, \mathbf{M}\right)} \stackrel{\text { q.s }}{\longrightarrow} A_{t}$ para cada $t \in[0, r]$. Vamos provar a martingalidade de $\mathbf{M}^{2}-\mathbf{A}$. Seja $t \in[0, r]$, temos que

$$
\mathbf{E}\left(M_{r}^{2}-Q_{r}^{\left(\mathcal{P}_{n}, \mathbf{M}\right)} \| \mathscr{F}_{t}\right)=M_{t}^{2}-Q_{t}^{\left(\mathcal{P}_{n}, \mathbf{M}\right)}, \quad \forall n \geq 1
$$

Temos que $Q_{r}^{\left(\mathcal{P}_{n}, \mathbf{M}\right)} \stackrel{\text { q.s }}{\longrightarrow} A_{r}$, mas como $\left\{Q^{\left(\mathcal{P}_{n}, \mathbf{M}\right)}(r)_{n}\right\}_{n \geq 1}$ também converge em $\mathrm{L}^{2}$, temos que $A_{r} \in \mathrm{L}^{2}$ e portanto vale também que $Q_{r}^{\left(\mathcal{P}_{n}, \mathrm{M}\right)} \stackrel{\mathrm{L}^{2}}{\rightarrow} A_{r}$ (em particular em $\mathrm{L}^{1}$ também). Assim*

$$
\mathbf{E}\left(M_{r}^{2}-Q_{r}^{\left(\mathcal{P}_{n}, \mathbf{M}\right)} \| \mathscr{F}_{t}\right) \stackrel{\mathrm{L}^{1}}{\rightarrow} \mathbf{E}\left(M_{r}^{2}-A_{r} \| \mathscr{F}_{t}\right) .
$$

Note que isso implica ${ }^{\dagger}$ também a convergência P-q. s. para uma subsequência apropriada de $\left\{\mathcal{P}_{n}\right\}_{n \geq 1}$.

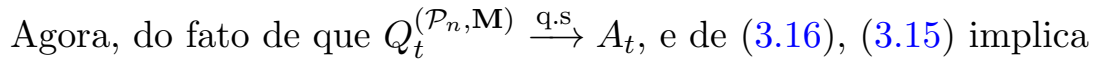

$$
\mathbf{E}\left(M_{r}^{2}-A_{r} \| \mathscr{F}_{t}\right)=M_{t}^{2}-A_{t}, \quad \mathrm{P}-\mathrm{q} . \mathrm{s} .
$$

Com isso concluimos que $\mathbf{M}^{2}-\mathbf{A}$ é um martingal no intervalo $[0, r]$. Com respeito à natureza crescente das trajetórias de $\mathbf{A}$, note que para $s<t, s, t \in \mathcal{P}$, então segue pela definição que $Q_{s}^{(\mathcal{P}, \mathbf{M})} \leq Q_{t}^{(\mathcal{P}, \mathbf{M})}$. Agora, defina $D \doteq \cup_{n} \mathcal{P} \subseteq[0, r]$, e tome $s<t$, com $s, t \in D$. Temos que existe $n_{0}$ tal que $s, t \in \mathcal{P}_{n}$ para todo $n \geq n_{0}$. Para tais $n$ temos que $Q_{s}^{\left(\mathcal{P}_{n}, \mathbf{M}\right)} \leq Q_{t}^{\left(\mathcal{P}_{n}, \mathbf{M}\right)}$ e fazendo $n \uparrow \infty, A_{s} \leq A_{t}$, P-q. s. O conjunto de medida zero onde isso não vale poderia depender de $s$ e $t$, mas como $D$ é enumerável $A_{s} \leq A_{t}$ para todos $s, t \in D$ com $s<t$, P-q. s., ou seja, quase toda trajetória $t \in[0, r] \mapsto A(t, \omega)$ é crescente em $D \subseteq[0, r]$. Pela continuidade das trajetórias e pela densidade de $D$, as trajetórias devem crescer em todo o intervalo $[0, r]$.

Finalmente, seja $t \in[0, r]$. Vimos que $B=\lim _{\left|\mathcal{P}_{n}\right| \rightarrow 0} Q^{(\mathcal{P}, \mathbf{M})}$ existe em $\mathrm{L}^{2}$ quando o limite é tomado sobre todas as partições $\mathcal{P}$ de $[0, t]$. Da nossa construção temos que $Q_{t}^{\left(\mathcal{P}_{n}, \mathbf{M}\right)} \stackrel{\text { q.s }}{\longrightarrow} A_{t}$, mas

\footnotetext{
* Veja o teorema C.15 na página 163. † Veja a observação após a proprosição B.2 na página 154.
} 
$Q_{t}^{\left(\mathcal{P}_{n}, \mathbf{M}\right)}=Q^{\left(\mathcal{P}_{n}(t), \mathbf{M}\right)}$ onde $\mathcal{P}_{n}(t)=\left(\mathcal{P}_{n} \cup\{t\}\right) \cap[0, t]$ é uma sequência de partições de $[0, t]$ com $\lim _{n \rightarrow \infty}\left|\mathcal{P}_{n}(t)\right| \rightarrow 0$. Dessa forma, $Q_{t}^{\left(\mathcal{P}_{n}, \mathbf{M}\right)} \stackrel{\mathrm{L}^{2}}{\rightarrow} B$ e assim, resulta

$$
A_{t}=B=\lim _{|\mathcal{P}| \rightarrow 0} Q^{(\mathcal{P}, \mathbf{M})} \text { em L }{ }^{2}, \text { P-q. s. }
$$

\subsection{Variação quadrática para martingais locais}

O objetivo agora é estender o conceito de variação quadrática para martingais locais. Afinal, pedir que o martingal seja uniformemente limitado é uma hipótese muito forte. Note que o movimento Browniano não é uniformemente limitado (pois $B_{t} \sim N(0, t)$ ). Antes, precisamos de um lema.

Lema 3.11. Seja $\mathbf{M}$ um martingal contínuo uniformemente limitado e $\tau$ um tempo de parada. Então

i) $\mathbf{E}\left(\llbracket \mathbf{M} \rrbracket_{t}\right)=\mathbf{E}\left(\left(M_{t}-M_{0}\right)^{2}\right) \quad \forall t \geq 0$.

ii) $\mathbf{M}^{\tau}$ é um martingal contínuo, uniformemente limitado e $\llbracket \mathbf{M}^{\tau} \rrbracket=\llbracket \mathbf{M} \rrbracket^{\tau}$.

Demonstração. i) Como $\mathbf{M}^{2}-\llbracket \mathbf{M} \rrbracket$ é um martingal e $\llbracket \mathbf{M} \rrbracket=0$,

$$
\mathbf{E}\left(M_{t}^{2}-\llbracket \mathbf{M} \rrbracket_{t}\right)=\mathbf{E}\left(M_{0}^{2}-\llbracket \mathbf{M} \rrbracket_{0}\right)=\mathbf{E}\left(M_{0}^{2}\right)
$$

e dessa forma,

$$
\mathbf{E}\left(\llbracket \mathbf{M} \rrbracket_{t}\right)=\mathbf{E}\left(M_{t}^{2}-M_{0}^{2}\right)
$$

agora pela proposição 3.3 resulta que

$$
\mathbf{E}\left(\llbracket \mathbf{M} \rrbracket_{t}\right)=\mathbf{E}\left(\left(M_{t}-M_{0}\right)^{2}\right)
$$

ii) Pelo corolário 2.38 na página 22, $\mathbf{M}^{\tau}$ é um martingal e como $\mathbf{M}$ é uniformemente limitado, $\mathbf{M}^{\tau}$ também o é. Dessa forma, a variação quadrática $\llbracket \mathbf{M}^{\tau} \rrbracket$ está bem definida e é o único processo de variação finita tal que $\left(\mathbf{M}^{\tau}\right)^{2}-\llbracket \mathbf{M}^{\tau} \rrbracket$ é um martingal. Porém, como $\mathbf{M}^{2}-\llbracket \mathbf{M} \rrbracket$ é martingal, usando 
novamente o corolário 2.38, $\left(\mathbf{M}^{2}-\llbracket \mathbf{M} \rrbracket\right)^{\tau}$ também é. Mas

$$
\begin{aligned}
\left(\mathbf{M}^{2}-\llbracket \mathbf{M} \rrbracket\right)^{\tau} & =\left(\mathbf{M}^{2}\right)^{\tau}-\llbracket \mathbf{M} \rrbracket^{\tau} \\
& =\left(\mathbf{M}^{\tau}\right)^{2}-\llbracket \mathbf{M} \rrbracket^{\tau}
\end{aligned}
$$

pois, para todo $t \geq 0$, vale que

$$
\left(M_{t}^{\tau}\right)^{2}=(M(\tau \wedge t))^{2}=M^{2}(t \wedge \tau)=\left(M_{t}^{2}\right)^{\tau}
$$

Como $\llbracket \mathbf{M} \rrbracket^{\tau}$ é contínuo, de variação finita, e $\left(\mathbf{M}^{\tau}\right)^{2}-\llbracket \mathbf{M} \rrbracket^{\tau}$ é martingal, resulta pela unicidade da variação quadrática que $\llbracket \mathbf{M}^{\tau} \rrbracket=\llbracket \mathbf{M} \rrbracket^{\tau}$, como queríamos.

Teorema 3.12. Seja $\mathbf{M}$ um martingal local contínuo. Então existe um único processo contínuo de variação finita A tal que $A(0)=0$ e $\mathbf{M}^{2}-\mathbf{A}$ é um martingal local. O processo $\mathbf{A}$ é chamado de variação quadrática de $\mathbf{M}$ e o denotamos por $\llbracket \mathbf{M} \rrbracket$. Além disso, $\llbracket \mathbf{M} \rrbracket$ é adaptado, crescente e

$$
\llbracket \mathbf{M} \rrbracket(t)=\lim _{|\mathcal{P}| \rightarrow 0} Q^{(\mathcal{P}, \mathbf{M})} \quad \text { em probabilidade, para cada } t \geq 0,
$$

onde o limite é tomado sobre todas as partições de $\mathcal{P}$ do intervalo $[0, t]$.

Demonstração. A prova da unicidade e a adaptabilidade é feita como no teorema 3.10. De acordo com a proposição 2.52 na página 30 , podemos escolher uma sequência redutora $\left\{\tau_{n}\right\}_{n \geq 1}$ tal que $\mathbf{Y}_{n} \doteq \mathbf{M}^{\tau_{n}} \mathbb{1}_{\left[\tau_{n}>0\right]}$ é um martingal contínuo e uniformemente limitado para cada $n \geq 1$. Pelo teorema 3.10, para cada $n \geq 1$, existe um único processo contínuo e crescente $\mathbf{A}_{n}$ tal que $\mathbf{Y}_{n}^{2}-\mathbf{A}_{n}$ é um martingal. Como $\tau_{n} \leq \tau_{n+1}$, temos que $\left[\tau_{n}>0\right] \subseteq\left[\tau_{n+1}>0\right]$ e portanto, $\mathbb{1}_{\left[\tau_{n}>0\right]} \mathbb{1}_{\left[\tau_{n+1}>0\right]}=\mathbb{1}_{\left[\tau_{n}>0\right]}$. Assim

$$
\begin{aligned}
\mathbf{Y}_{n}=\mathbf{M}^{\tau_{n}} \mathbb{1}_{\left[\tau_{n}>0\right]} & =\left(\mathbf{M}^{\tau_{n+1}}\right)^{\tau_{n}} \mathbb{1}_{\left[\tau_{n+1}>0\right]} \mathbb{1}_{\left[\tau_{n}>0\right]} \\
& =\left(\mathbf{M}^{\tau_{n+1}} \mathbb{1}_{\left[\tau_{n+1}>0\right]}\right)^{\tau_{n}} \mathbb{1}_{\left[\tau_{n}>0\right]} \\
& =\mathbf{Y}_{n+1}^{\tau_{n}} \mathbb{1}_{\left[\tau_{n}>0\right]} .
\end{aligned}
$$

Agora, fixe $n \geq 1$. Como $\mathbf{Y}_{n+1}^{2}-\mathbf{A}_{n+1}$ é um martingal, $\left(\mathbf{Y}_{n+1}^{2}-\mathbf{A}_{n+1}^{2}\right)^{\tau_{n}}=\left(\mathbf{Y}_{n+1}^{\tau_{n}}\right)^{2}-\mathbf{A}_{n+1}^{\tau_{n}}$ 
também é. Note que se $\mathbf{M}$ é um martingal e $F \in \mathscr{F}_{0}$, então $\mathbf{M} \mathbb{1}_{[F]}$ é martingal, logo

$$
\begin{aligned}
{\left[\left(\mathbf{Y}_{n+1}^{\tau_{n}}\right)^{2}-\mathbf{A}_{n+1}^{\tau_{n}}\right] \mathbb{1}_{\left[\tau_{n}>0\right]} } & =\left(\mathbf{Y}_{n+1}^{\tau_{n}} \mathbb{1}_{\left[\tau_{n}>0\right]}\right)^{2}-\mathbf{A}_{n+1}^{\tau_{n}} \mathbb{1}_{\left[\tau_{n}>0\right]} \\
& =\mathbf{Y}_{n}^{2}-\mathbf{A}_{n+1}^{\tau_{n}} \mathbb{1}_{\left[\tau_{n}>0\right]}
\end{aligned}
$$

é um martingal. Pela unicidade do processo $\mathbf{A}_{n}$, concluímos que $\mathbf{A}_{n}=\mathbf{A}_{n+1}^{\tau_{n}} \mathbb{1}_{\left[\tau_{n}>0\right]}, \forall(t, \omega) \in \mathbb{R}_{+} \times \Omega$. Assim, temos que $\mathbf{A}_{n}=\mathbf{A}_{n+1}$ no conjunto $\Pi \doteq\left\{(t, \omega) \in \mathbb{R}_{+} \times \Omega: \tau_{n}(\omega)>0\right.$ e $\left.t \leq \tau_{n}(\omega)\right\} \subseteq \mathbb{R}_{+} \times \Omega$. Como os conjuntos $\Pi_{n}$ são crescentes, temos que

$$
\mathbf{A}_{n}=\mathbf{A}_{n+1}=\mathbf{A}_{n+2}=\ldots \text { em } \Pi_{n} .
$$

Como $\Pi_{n} \uparrow \mathbb{R}_{+} \times \Omega$, podemos definir um processo $\mathbf{A}$ em $\mathbb{R}_{+} \times \Omega$ definindo $\mathbf{A}=\mathbf{A}_{n}$ em $\Pi_{n}, n \geq 1$. Agora fixe $t \geq 0$. Temos que $A(t, \omega)=A_{n}(t, \omega)$, para todo $n \geq 1$ tal que $\tau_{n}(\omega)>0$ e $t \leq \tau_{n}(\omega)$, isto é, para todos exceto, no máximo, um número finito de índices $n$. Então $A(t, \omega)=\lim _{n \rightarrow \infty} A_{n}(t, \omega)$ e portanto, $A(t, \cdot) \in \mathscr{F}_{t}$. Pela definição de $\mathbf{A}$, segue que

$$
\mathbf{A}^{\tau_{n}} \mathbb{1}_{\left[\tau_{n}>0\right]}=\mathbf{A}_{n}^{\tau_{n}} \mathbb{1}_{\left[\tau_{n}>0\right]}=\mathbf{A}_{n}
$$

$\operatorname{assim}, \mathbf{Y}_{n}^{2}-\mathbf{A}_{n}=\left(\mathbf{M}^{\tau_{n}} \mathbb{1}_{\left[\tau_{n}>0\right]}\right)^{2}-\mathbf{A}^{\tau_{n}}\left(\mathbb{1}_{\left[\tau_{n}>0\right]}\right)=\left(\mathbf{M}^{2}-\mathbf{A}\right)^{\tau_{n}} \mathbb{1}_{\left[\tau_{n}>0\right]}$. Como $\mathbf{Y}_{n}^{2}-\mathbf{A}_{n}$ é um martingal, resulta que $\left(\mathbf{M}^{2}-\mathbf{A}\right)^{\tau_{n}} \mathbb{1}_{\left[\tau_{n}>0\right]}$ também é e, portanto, provamos que $\mathbf{M}^{2}-\mathbf{A}$ é um martingal local. Como $\mathbf{A}_{n}$ é contínuo e de variação finita, segue de (3.17) que $\mathbf{A}^{\tau_{n}>0} \mathbb{1}_{\left[\tau_{n}>0>0\right]}$ também é para cada $n \geq 1$. Resulta portanto que $\mathbf{A}$ tem a mesma propriedade e além disso, $A(0)=\lim _{n \rightarrow \infty} A_{n}(0)=0$, e isso prova a existência de $\mathbf{A}$.

Agora, fixe $t \geq 0$ e $\mathcal{P}$ um partição qualquer de $[0, t]$. Pelo teorema 3.10 , temos que $Q^{\mathcal{P}, \mathbf{Y}_{n}}(t) \stackrel{\mathrm{L}^{2}}{\longrightarrow}$ $A_{n}(t)$ e portanto em probabilidade. Como $\mathbf{Y}_{n}=\mathbf{M}$ e $\mathbf{A}_{n}=\mathbf{A}$ em $\Pi_{n} \subseteq \mathbb{R}_{+} \times \Omega$, o processo $Q^{\left(\mathcal{P}, \mathbf{Y}_{n}\right)}(\cdot)$ satisfaz $Q^{\left(\mathcal{P}, \mathbf{Y}_{n}\right)}(\cdot)=Q^{(\mathcal{P}, \mathbf{Y})}(\cdot)$ em $\Pi_{n}$ também. Logo $Q^{\left(\mathcal{P}, \mathbf{Y}_{n}\right)}(t)=Q^{(\mathcal{P}, \mathbf{M})}$ e $A_{n}(t)=A(t)$ em $\Omega_{n}^{+}=\left[\tau_{n}>0\right] \cap\left[t \leq \tau_{n}\right]$. Temos portanto que para $|\mathcal{P}| \rightarrow 0, Q^{(\mathcal{P}, \mathbf{M})}(t) \stackrel{\mathrm{P}}{\rightarrow} A(t)$ em cada $\Omega_{n}$. Como $\Omega_{n} \uparrow \Omega$, temos pela proposição B.3 na página 154 , que a convergência em probabilidade acima se dá em todo $\Omega$.

Agora, vamos estudar algumas propriedades da variação quadrática.

Proposição 3.13. Seja $\mathbf{M}$ um martingal local contínuo e $\tau$ um tempo de parada. Então 
i) $\llbracket \mathbf{M}^{\tau} \rrbracket=\llbracket \mathbf{M} \rrbracket^{\tau}$.

ii) Se $F \in \mathscr{F}_{0}$ então $\llbracket \mathbb{1}_{[F]} \mathbf{M} \rrbracket=\mathbb{1}_{[F]} \llbracket \mathbf{M} \rrbracket$.

iii) $\llbracket \mathbf{M} \rrbracket=0$ se, e somente se, $\mathbf{M}$ for constante (no tempo).

Demonstração. i) Como $\mathbf{M}^{2}-\llbracket \mathbf{M} \rrbracket$ é um martingal local, pela proposição 2.49 (item iii)), temos que $\left(\mathbf{M}^{2}-\llbracket \mathbf{M} \rrbracket\right)^{\tau}$ também é um martingal local. Agora como

$$
\left(\mathbf{M}^{2}-\llbracket \mathbf{M} \rrbracket\right)^{\tau}=\left(\mathbf{M}^{2}\right)^{\tau}-\llbracket \mathbf{M} \rrbracket^{\tau}=\left(\mathbf{M}^{\tau}\right)^{2}-\llbracket \mathbf{M} \rrbracket^{\tau}
$$

concluímos também que $\left(\mathbf{M}^{\tau}\right)^{2}-\llbracket \mathbf{M} \rrbracket^{\tau}$ é martingal local e $\llbracket \mathbf{M} \rrbracket^{\tau}$ é contínuo (pois $\mathbf{M}$ é) e de variação limitada (pois $\llbracket \mathbf{M} \rrbracket$ é). Porém, note que como $\mathbf{M}^{\tau}$ é martingal local contínuo, então, pelo teorema anterior, existe e é único o processo contínuo e de variação finita $\llbracket \mathbf{M}^{\tau} \rrbracket$ tal que

$$
\left(\mathbf{M}^{\tau}\right)^{2}-\llbracket \mathbf{M}^{\tau} \rrbracket
$$

é um martingal local. Dessa unicidade, resulta que $\llbracket \mathbf{M}^{\tau} \rrbracket=\llbracket \mathbf{M} \rrbracket^{\tau}$.

ii) Fixe $t \geq 0$ e seja $\mathcal{P}$ uma partição de $[0, t]$. Temos pela proposição 2.49 (item ii)) que $\mathbb{1}_{[F]} \mathbf{M}$ também é martingal local e assim, pelo teorema anterior, temos que em probabilidade, vale

$$
\llbracket \mathbb{1}_{[F]} \mathbf{M} \rrbracket_{t}=\lim _{|\mathcal{P}| \rightarrow 0} Q^{\left(\mathcal{P}, \mathbb{1}_{[F]} \mathbf{M}\right)}
$$

Mas, lembre-se que se $\mathcal{P}: 0=t_{0}<t_{1}<\ldots<t_{n}=t$, então

$$
\begin{aligned}
Q^{\left(\mathcal{P}, \mathbb{1}_{[F]} \mathbf{M}\right)} & =\sum_{j=1}^{n}\left(\mathbb{1}_{[F]} M_{t_{j}}-\mathbb{1}_{[F]} M_{t_{j-1}}\right)^{2} \\
& =\mathbb{1}_{[F]} \sum_{j=1}^{n}\left(M_{t_{j}}-M_{t_{j-1}}\right)^{2} \\
& =\mathbb{1}_{[F]} Q^{\left(\mathcal{P}, \mathbb{1}_{[F]} \mathbf{M}\right)} .
\end{aligned}
$$

Disso, resulta

$$
\llbracket \mathbb{1}_{[F]} \mathbf{M} \rrbracket_{t}=\lim _{|\mathcal{P}| \rightarrow 0} Q^{\left(\mathcal{P}, \mathbb{1}_{[F]} \mathbf{M}\right)}=\mathbb{1}_{[F]} \lim _{|\mathcal{P}| \rightarrow 0} Q^{(\mathcal{P}, \mathbf{M})}=\mathbb{1}_{[F]} \llbracket \mathbf{M} \rrbracket_{t} .
$$

Da arbitrariedade de $t$, segue o resultado. 
iii) Suponha que $M_{t}=M_{0}$, para todo $t \geq 0$, então $\mathbf{M}^{2}$ é um martingal local, o que nos força a concluir que $\llbracket \mathbf{M} \rrbracket=0$. Reciprocamente suponha que $\llbracket \mathbf{M} \rrbracket=0$. Temos que $\llbracket \mathbf{M}^{\tau} \rrbracket=\llbracket \mathbf{M} \rrbracket^{\tau}=0$ para qualquer tempo de parada. Pela proposição 2.52, podemos assumir que existe uma sequência redutora $\left\{\tau_{n}\right\}_{n \geq 1}$ para $\mathbf{M}$ de tal forma que $\mathbf{Y}_{n} \doteq \mathbf{M}^{\tau_{n}} \mathbb{1}_{\left[\tau_{n}>0\right]}$ seja um martingal uniformemente limitado. Agora, fixe $t \geq 0$, arbitrário, pelo lema 3.11 (item i)), temos que para todo $n \geq 1$, vale

$$
\begin{aligned}
\mathbf{E}\left(\left(Y_{n}(t)-Y_{n}(0)\right)^{2}\right) & =\mathbf{E}\left(\llbracket \mathbf{Y}_{\mathbf{n}} \rrbracket(t)\right) \\
& =\mathbf{E}\left(\llbracket \mathbf{M}^{\tau_{\mathbf{n}}} \mathbb{1}_{\left[\tau_{\mathbf{n}}>\mathbf{0}\right] \rrbracket} \rrbracket(t)\right) \\
& =\mathbf{E}\left(\mathbb{1}_{\left[\tau_{n}>0\right]} \llbracket \mathbf{M}^{\tau_{\mathbf{n}}} \rrbracket(t)\right) \\
& =\mathbf{E}\left(\mathbb{1}_{\left[\tau_{n}>0\right]} \llbracket \mathbf{M} \rrbracket^{\tau_{n}}(t)\right) \\
& =\mathbf{E}\left(\mathbb{1}_{\left[\tau_{n}>0\right]} \cdot 0\right)=0 .
\end{aligned}
$$

onde na terceira igualdade usamos o item ii), na quarta, o item i) e na quinta usamos a hipótese que $\llbracket \mathbf{M} \rrbracket^{\tau}=0$. Assim, ficamos com

$$
\mathbf{E}\left(\left(Y_{n}(t)-Y_{n}(0)\right)^{2}\right)=0
$$

e portanto $Y_{n}(t)=Y_{n}(0)$, P-q. s., ou seja $M^{\tau_{n}} \mathbb{1}_{\left[\tau_{n}>0\right]}(t)=M^{\tau_{n}} \mathbb{1}_{\left[\tau_{n}>0\right]}(0)$, P-q. s., $\forall n \geq 1$. Mas, como $\tau_{n} \nearrow \infty$, P-q.s. , $\mathbb{1}_{\left[\tau_{n}>0\right]} \stackrel{\text { q.s }}{\longrightarrow} \mathbb{1}_{[\omega]}$ e $M^{\tau_{n}}(t) \stackrel{\text { q.s }}{\longrightarrow} M(t)$ assim, resulta que $M(t)=M(0)$, P-q. s.. Da arbitrariedade de $t$ segue que $\mathbf{M}$ é uma modificação do processo estocástico constante $\mathbf{M}_{0}=M(0, \omega)$ e pela continuidade de $\mathbf{M}$, segue que pela proposição 2.3 que $\mathbf{M}$ é indistinguível de uma constante. Como queríamos.

Exemplo 3.14. A variação quadrática do movimento Browniano padrão é tal que $\llbracket \mathbf{B} \rrbracket_{t}=t$, para todo $t \geq 0$. Para ver isto, note que o movimento Browniano é um martingal, e portanto é um martingal local* então, pela unicidade da variação quadrática dada pelo teorema 3.12 , basta mostrar que $\left\{B_{t}^{2}-t\right\}_{t \geq 0}$ é um martingal. Para isto, seja $0 \leq s<t$. Devemos mostrar que $\mathbf{E}\left(B_{t}^{2}-t \| \mathscr{F}_{s}\right)=B_{s}^{2}-t$

\footnotetext{
* Veja o exemplo 2.26 na página 18.
} 
ou, de forma equivalente, $\mathbf{E}\left(B_{t}^{2}-B_{s}^{2} \| \mathscr{F}_{s}\right)=t-s$. De fato,

$$
\begin{aligned}
\mathbf{E}\left(B_{t}^{2}-B_{s}^{2} \| \mathscr{F}_{s}\right) & =\mathbf{E}\left(\left(B_{t}-B_{s}\right)^{2} \| \mathscr{F}_{s}\right) \\
& =\mathbf{E}\left(\left(B_{t}-B_{s}\right)^{2}\right) \\
& =\operatorname{Var}\left(B_{t}-B_{s}\right) \\
& =t-s,
\end{aligned}
$$

onde a primeira igualdade vale pela proprosição 3.3 , a segunda pelo fato de $B_{t}-B_{s}$ ser independente de $\mathscr{F}_{s}$ e a última pelo fato do incremento do movimento Browniano ter variância $t-s$.

\subsection{Covariação quadrática}

Definição 3.15. Sejam $\mathbf{M}$ e $\mathbf{N}$ martingais locais contínuos. Definimos a covariação quadrática entre $\mathbf{M}$ e $\mathbf{N}$ pela expressão

$$
\llbracket \mathbf{M}, \mathbf{N} \rrbracket \doteq \frac{1}{4}(\llbracket \mathbf{M}+\mathbf{N} \rrbracket-\llbracket \mathbf{M}-\mathbf{N} \rrbracket)
$$

O próximo resultado mostra que a covariação quadrática é de fato uma extensão do conceito de variação quadrática para dois processo estocásticos.

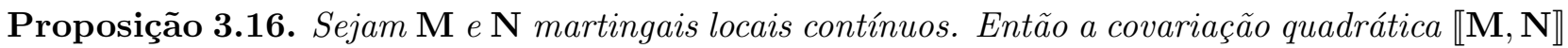
é o único processo contínuo e de variação finita tal que $\llbracket \mathbf{M}, \mathbf{N} \rrbracket_{0}=0$ e $\mathbf{M N}-\llbracket \mathbf{M}, \mathbf{N} \rrbracket$ é um martingal local.

Demonstração. Como $\mathbf{M}+\mathbf{N}$ é martingal local contínuo, pelo teorema 3.12, $\llbracket \mathbf{M}+\mathbf{N} \rrbracket$ e $\llbracket \mathbf{M}-\mathbf{N} \rrbracket$ são contínuos, de variação finita e nulos em $t=0$ e portanto, por (3.18), o mesmo vale para $\llbracket \mathbf{M}, \mathbf{N} \rrbracket$. Além disso, $\llbracket \mathbf{M}+\mathbf{N} \rrbracket$ e $\llbracket \mathbf{M}-\mathbf{N} \rrbracket$ são os únicos processos com essas características e tais que $(\mathbf{M}+\mathbf{N})^{2}-$ $\llbracket \mathbf{M}+\mathbf{N} \rrbracket$ e $(\mathbf{M}-\mathbf{N})^{2}-\llbracket \mathbf{M}-\mathbf{N} \rrbracket$ são martingais locais. Agora, da a identidade algébrica

$$
\mathbf{M N}=\frac{1}{4}\left[(\mathbf{M}+\mathbf{N})^{2}-(\mathbf{M}-\mathbf{N})^{2}\right]
$$

temos que

$$
\mathbf{M N}-\llbracket \mathbf{M}, \mathbf{N} \rrbracket=\frac{1}{4}\left\{\left[(\mathbf{M}+\mathbf{N})^{2}-\llbracket \mathbf{M}+\mathbf{N} \rrbracket\right]-\left[(\mathbf{M}-\mathbf{N})^{2}-\llbracket \mathbf{M}-\mathbf{N} \rrbracket\right]\right\}
$$


e portanto $\mathbf{M N}-\llbracket \mathbf{M}, \mathbf{N} \rrbracket$ é um martingal local. Como queríamos.

Como consequência direta deste resultado temos o seguinte

Corolário 3.17. Sejam $\mathbf{M}$ e $\mathbf{N}$ martingais locais contínuos. Então $\mathbf{M N}$ é martingal local se $e$ somente se $\llbracket \mathbf{M}, \mathbf{N} \rrbracket=0$.

Assim como nos teoremas 3.12 e 3.10, também temos uma representação por limites para a covariação quadrática.

Proposição 3.18. Sejam $\mathbf{M}$ e $\mathbf{N}$ martingais locais contínuos. Para cada $t>0$ e cada partição $\mathcal{P}: 0=t_{0}<t_{1}<\ldots<t_{n}=t$ do intervalo $[0, t]$, defina a variável aleatória

$$
Q^{(\mathcal{P}, \mathbf{M}, \mathbf{N})} \doteq \sum_{j=1}^{n}\left(M_{t_{j}}-M_{t_{j-1}}\right)\left(N_{t_{j}}-N_{t_{j-1}}\right)
$$

Então, para $|\mathcal{P}| \rightarrow 0$

$$
Q^{(\mathcal{P}, \mathbf{M}, \mathbf{N})} \stackrel{\mathrm{P}}{\rightarrow} \llbracket \mathbf{M}, \mathbf{N} \rrbracket_{t}
$$

e se $\mathbf{M}$ e $\mathbf{N}$ forem martingais uniformemente limitados, a convergência se dá em $\mathrm{L}^{2}$ também.

Demonstração. Em primeiro lugar, note que usando a identidade

$$
\mathbf{M N}=\frac{1}{4}\left[(\mathbf{M}+\mathbf{N})^{2}-(\mathbf{M}-\mathbf{N})^{2}\right]
$$

temos que

$$
Q^{(\mathcal{P}, \mathbf{M}, \mathbf{N})}=\frac{1}{4}\left(Q^{(\mathcal{P}, \mathbf{M}+\mathbf{N})}-Q^{(\mathcal{P}, \mathbf{M}-\mathbf{N})}\right)
$$

e o resultado segue pelos teoremas 3.12 e 3.10

Vamos agora mostrar algumas propriedades da covariação quadrática.

Proposição 3.19. Sejam $\mathbf{M}, \mathbf{N}$ e $\mathbf{U}$ martingais locais contínuos, $\xi$ e $\eta$ variáveis aleatórias $\mathscr{F}_{0}{ }^{-}$ mensuráveis. Então

$$
\llbracket \xi \mathbf{M}+\eta \mathbf{N}, \mathbf{U} \rrbracket=\xi \llbracket \mathbf{M}, \mathbf{U} \rrbracket+\eta \llbracket \mathbf{N}, \mathbf{U} \rrbracket .
$$


Demonstração. Vamos provar inicialmente que vale

$$
\llbracket \mathbf{M}+\mathbf{N}, \mathbf{U} \rrbracket=\llbracket \mathbf{M}, \mathbf{U} \rrbracket+\llbracket \mathbf{N}, \mathbf{U} \rrbracket .
$$

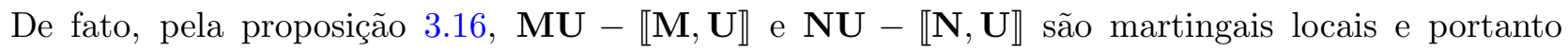
$(\mathbf{M}+\mathbf{N}) \mathbf{U}-(\llbracket \mathbf{M}, \mathbf{U} \rrbracket-\llbracket \mathbf{N}, \mathbf{U} \rrbracket)$ também o é. No entanto, novamente pela proposição 3.16, também temos que $(\mathbf{M}+\mathbf{N}) \mathbf{U}-\llbracket \mathbf{M}+\mathbf{N}$, U』é martingal local contínuo e portanto, pela unicidade da covariação quadrática, resulta (3.20). Para provar (3.19) basta agora demonstrar que $\llbracket \xi \mathbf{M}, \mathbf{N} \rrbracket=$ $\xi \llbracket \mathbf{M}, \mathbf{N} \rrbracket$. De fato, como $\mathbf{M U}-\llbracket \mathbf{M}, \mathbf{U} \rrbracket$ é martingal local e $\xi$ é $\mathscr{F}_{0}$-mensurável, $\xi(\mathbf{M U}-\llbracket \mathbf{M}, \mathbf{U} \rrbracket)=$ $\xi \mathbf{M U}-\xi \llbracket \mathbf{M}, \mathbf{U} \rrbracket$ também é martingal local. Mas como $\xi \mathbf{M}$ e $\mathbf{N}$ são martingais locais contínuos, pela

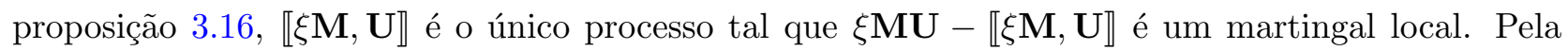
unicidade da covariação quadrática, temos que vale (3.19).

É fácil ver que a covariação quadrática é simetrica, isto é, $\llbracket \mathbf{M}, \mathbf{N} \rrbracket=\llbracket \mathbf{N}, \mathbf{M} \rrbracket$ e portanto, deste último resultado, segue que a covariação quadrática é bilinear. A última propriedade que vamos provar é dada na seguinte

Proposição 3.20. Sejam $\tau$ um tempo de parada, $\mathbf{M}$ e $\mathbf{N}$ martingais locais contínuos. Então

$$
\llbracket \mathbf{M}^{\tau}, \mathbf{N}^{\tau} \rrbracket=\llbracket \mathbf{M}, \mathbf{N} \rrbracket^{\tau}=\llbracket \mathbf{M}^{\tau}, \mathbf{N} \rrbracket .
$$

Demonstração. De acordo com a proposição 3.13, item i), temos que

$$
\begin{aligned}
\llbracket \mathbf{M}^{\tau}, \mathbf{N}^{\tau} \rrbracket & =\frac{1}{4}\left(\llbracket(\mathbf{M}+\mathbf{N})^{\tau} \rrbracket-\llbracket(\mathbf{M}-\mathbf{N})^{\tau} \rrbracket\right) \\
& =\frac{1}{4}\left(\llbracket \mathbf{M}+\mathbf{N} \rrbracket^{\tau}-\llbracket \mathbf{M}-\mathbf{N} \rrbracket^{\tau}\right) \\
& =\frac{1}{4}(\llbracket \mathbf{M}+\mathbf{N} \rrbracket-\llbracket \mathbf{M}-\mathbf{N} \rrbracket)^{\tau} \\
& =\llbracket \mathbf{M}, \mathbf{N} \rrbracket^{\tau} .
\end{aligned}
$$

Vamos mostrar agora que $\llbracket \mathbf{M}^{\tau}, \mathbf{N}^{\tau} \rrbracket=\llbracket \mathbf{M}^{\tau}, \mathbf{N} \rrbracket$. Para isso, perceba que é necessário e suficiente provar que $\llbracket \mathbf{M}^{\tau}, \mathbf{N}-\mathbf{N}^{\tau} \rrbracket=0$. De fato, $\llbracket \mathbf{M}^{\tau}, \mathbf{N}^{\tau} \rrbracket=\llbracket \mathbf{M}^{\tau}, \mathbf{N} \rrbracket \Leftrightarrow \llbracket \mathbf{M}^{\tau}, \mathbf{N}^{\tau} \rrbracket-\llbracket \mathbf{M}^{\tau}, \mathbf{N} \rrbracket=0 \Leftrightarrow$ $\llbracket \mathbf{M}^{\tau}, \mathbf{N}-\mathbf{N}^{\tau} \rrbracket=0$. Agora, fixe $\omega \in \Omega$. Note que as trajétorias $s \mapsto M^{\tau}(s, \omega)$ são constantes para $s \geq \tau(\omega)$ e as trajétorias $s \mapsto\left(N-N^{\tau}\right)(s, \omega)$ são identicamente nulas para $s \leq \tau(\omega)$. Agora, fixe $t \geq 0$ e seja $\mathcal{P}: 0=t_{0}<t_{1}<\ldots<t_{n}=t$, uma partição do intervalo [0,t]. Para facilitar a notação 
seja $\mathbf{Z} \doteq \mathbf{X}^{\tau}$ e $\mathbf{W} \doteq \mathbf{Y}-\mathbf{Y}^{\tau}$ Se $\omega \in \Omega$ for tal que $t<\tau(\omega)$, então $Q^{(\mathcal{P}, \mathbf{Z}, \mathbf{W})}(\omega)=0$, para toda partição $\mathcal{P}$ de $[0, t]$ e portanto $\lim _{n \rightarrow \infty} Q^{(\mathcal{P}, \mathbf{Z}, \mathbf{W})}(\omega)=0$. Caso $\omega \in \Omega$ for tal que $t \geq \tau(\omega)$, então

$$
Q^{(\mathcal{P}, \mathbf{Z}, \mathbf{W})}(\omega)=\left[Z_{t_{j}}(\omega)-Z_{t_{j-1}}(\omega)\right]\left[W_{t_{j}}(\omega)-W_{t_{j-1}}(\omega)\right]
$$

onde $j$ é tal que $t_{j-1}<\tau(\omega) \leq t_{j}$. Pela continuidade dos processos, temos que para $|\mathcal{P}| \rightarrow 0$, $\lim _{|\mathcal{P}| \rightarrow 0} Q^{(\mathcal{P}, \mathbf{Z}, \mathbf{W})}(\omega)=0$ e assim temos que $\lim _{|\mathcal{P}| \rightarrow 0} Q^{(\mathcal{P}, \mathbf{Z}, \mathbf{W})}(\omega)=0$ para todo $\omega \in \Omega$. Como o convergência quase certa implica na convergência em probabilidade, temos que

$$
\lim _{|\mathcal{P}| \rightarrow 0} Q^{(\mathcal{P}, \mathbf{Z}, \mathbf{W})}=\llbracket \mathbf{Z}^{\tau}, \mathbf{W}-\mathbf{W}^{\tau} \rrbracket=0
$$

como queríamos.

\subsection{Variação quadrática de martingais limitados em $L^{2}$}

Um martingal contínuo limitado em $\mathrm{L}^{2}$ não é necessariamente uniformemente limitado. Dessa forma, sua variação quadrática deve ser calculada no mesmo sentido da variação quadrática de um martingal local. O próximo resultado mostra um uso da variação quadrática como ferramenta para identificar martingais contínuos limitados em $\mathrm{L}^{2}$.

Proposição 3.21. Seja $\mathbf{M}$ um martingal local contínuo. São equivalentes

i) $\mathbf{M}$ é limitado em $\mathrm{L}^{2}$;

ii) $M(0) \in \mathrm{L}^{2}$ e $\mathbf{E}(\llbracket \mathbf{M} \rrbracket(\infty))<\infty$.

Em ambos os casos, $\mathbf{M}^{2}-\llbracket \mathbf{M} \rrbracket e ́$ um martingal uniformemente limitado.

Demonstração. Inicialmente, vimos* que podemos escolher uma sequência redutora para $\mathbf{M}$ de tal forma que $\mathbf{X}_{n} \doteq \mathbf{M}^{\tau_{n}} \mathbb{1}_{\left[\tau_{n}>0\right]}$ seja um martingal uniformemente limitado para cada $n \geq 1$. Assim, pelo teorema 3.10, $Z(t) \doteq\left(X_{n}(t)\right)^{2}-\llbracket \mathbf{X}_{\mathbf{n}} \rrbracket(t)$ é um martingal e portanto, tem média constante, isto é, $\mathbf{E}(Z(t))=\mathbf{E}(Z(0))$. Agora, pela proposição 3.13 temos que $\llbracket \mathbf{X}_{\mathbf{n}} \rrbracket=\mathbb{1}_{\left[\tau_{n}>0\right]} \llbracket \mathbf{M}_{\mathbf{n}} \rrbracket^{\tau_{n}}$ e portanto da igualdade $\mathbf{E}(Z(t))=\mathbf{E}(Z(0))$ temos que

$$
\mathbf{E}\left(M^{2}\left(t \wedge \tau_{n}\right) \mathbb{1}_{\left[\tau_{n}>0\right]}\right)-\mathbf{E}\left(\llbracket \mathbf{M} \rrbracket\left(t \wedge \tau_{n}\right) \mathbb{1}_{\left[\tau_{n}>0\right]}\right)=\mathbf{E}\left(M^{2}(0) \mathbb{1}_{\left[\tau_{n}>0\right]}\right) .
$$

\footnotetext{
* Veja a proposição 2.52 na página 30.
} 
$\mathbf{i} \Rightarrow$ ii: Como $\mathbf{M} \in \mathcal{H}^{2}$ é imediato que $M(0) \in \mathrm{L}^{2}$. Agora, de (3.21) temos que

$$
\mathbf{E}\left(\llbracket \mathbf{M} \rrbracket\left(t \wedge \tau_{n}\right) \mathbb{1}_{\left[\tau_{n}>0\right]}\right)=\mathbf{E}\left(M^{2}\left(t \wedge \tau_{n}\right) \mathbb{1}_{\left[\tau_{n}>0\right]}\right)-\mathbf{E}\left(M^{2}(0) \mathbb{1}_{\left[\tau_{n}>0\right]}\right) .
$$

Fixado $t \geq 0$, temos para cada $n \geq 1$ que $\left\{\llbracket \mathbf{M} \rrbracket\left(t \wedge \tau_{n}\right) \mathbb{1}_{\left[\tau_{n}>0\right]}\right\}_{n}$ é uma sequência crescente de funções mensuráveis tais que $\llbracket \mathbf{M} \rrbracket\left(t \wedge \tau_{n}\right) \mathbb{1}_{\left[\tau_{n}>0\right]} \leq \llbracket \mathbf{M} \rrbracket(t)$, P-q. s., para todo $n \geq 1$. Então, pelo teorema da convergência monótona,

$$
\lim _{n \rightarrow \infty} \mathbf{E}\left(\llbracket \mathbf{M} \rrbracket\left(t \wedge \tau_{n}\right) \mathbb{1}_{\left[\tau_{n}>0\right]}\right)=\mathbf{E}(\llbracket \mathbf{M} \rrbracket(t)) .
$$

Além disso, pela desigualdade de Doob*

$$
\mathbf{E}\left(\sup _{t \geq 0}|M(t)|^{2}\right) \leq 4 \sup _{t \geq 0} \mathbf{E}\left(M^{2}(t)\right) \doteq 4\|M\|_{\mathcal{H}^{2}}^{2}<\infty
$$

ou seja,

$$
M^{2}\left(\tau_{n} \wedge t\right) \mathbb{1}_{\left[\tau_{n}>0\right]} \leq \sup _{t \geq 0}|M(t)|^{2} \in \mathrm{L}^{1}, \forall n \geq 1 .
$$

Assim, pelo teorema da convergência dominada,

$$
\lim _{n \rightarrow \infty} \mathbf{E}\left(M^{2}\left(\tau_{n} \wedge t\right) \mathbb{1}_{\left[\tau_{n}>0\right]}\right)=\mathbf{E}\left(\lim _{n \rightarrow \infty} M^{2}\left(\tau_{n} \wedge t\right) \mathbb{1}_{\left[\tau_{n}>0\right]}\right)=\mathbf{E}\left(M^{2}(t)\right) .
$$

Usando (3.22), (3.23) e (3.24) ficamos com

$$
\mathbf{E}(\llbracket \mathbf{M} \rrbracket(t))=\mathbf{E}\left(M^{2}(t)\right)-\mathbf{E}\left(M^{2}(0)\right) .
$$

Como $\llbracket \mathbf{M} \rrbracket(\cdot)$ é crescente, novamente, pelo teorema da convergência monótona,

$$
\begin{aligned}
\lim _{t \rightarrow \infty} \mathbf{E}(\llbracket \mathbf{M} \rrbracket(t)) & =\mathbf{E}(\llbracket \mathbf{M} \rrbracket(\infty)) \\
& =\lim _{t \rightarrow \infty} \mathbf{E}\left(M^{2}(t)\right)-\mathbf{E}\left(M^{2}(0)\right) \\
& =\sup _{t \geq 0} \mathbf{E}\left(M^{2}(t)\right)-\mathbf{E}\left(M^{2}(0)\right) \\
& =\|M\|_{\mathcal{H}^{2}}^{2}-\mathbf{E}\left(M^{2}(0)\right)<\infty .
\end{aligned}
$$

Como queríamos.

* Veja o teorema 2.27 na página 18. 
$\mathbf{i i} \Rightarrow \mathbf{i}$ : Vamos mostrar que $\mathbf{M}$ é limitado em $\mathrm{L}^{2}$. De fato, de (3.21), temos que

$$
\begin{aligned}
\mathbf{E}\left(M^{2}\left(t \wedge \tau_{n}\right) \mathbb{1}_{\left[\tau_{n}>0\right]}\right) & =\mathbf{E}\left(\llbracket \mathbf{M} \rrbracket\left(t \wedge \tau_{n}\right) \mathbb{1}_{\left[\tau_{n}>0\right]}\right)+\mathbf{E}\left(M^{2}(0) \mathbb{1}_{\left[\tau_{n}>0\right]}\right) \\
& \leq \mathbf{E}(\llbracket \mathbf{M} \rrbracket(\infty))+\mathbf{E}\left(M^{2}(0)\right)<\infty .
\end{aligned}
$$

Agora, pelo lema de Fatou temos, para todo $t \geq 0$,

$$
\begin{aligned}
\mathbf{E}\left(M^{2}(t)\right) & =\mathbf{E}\left(\liminf _{n} M^{2}\left(t \wedge \tau_{n}\right) \mathbb{1}_{\left[\tau_{n}>0\right]}\right) \\
& \leq \liminf _{n} \mathbf{E}\left(M^{2}\left(t \wedge \tau_{n}\right) \mathbb{1}_{\left[\tau_{n}>0\right]}\right) \\
& \leq \mathbf{E}(\llbracket \mathbf{M} \rrbracket(\infty))+\mathbf{E}\left(M^{2}(0)\right)
\end{aligned}
$$

e portanto

$$
\sup _{t \geq 0} \mathbf{E}\left(M^{2}(t)\right) \leq \mathbf{E}(\llbracket \mathbf{M} \rrbracket(\infty))+\mathbf{E}\left(M^{2}(0)\right)<\infty .
$$

Agora vamos mostrar que $\mathbf{M}$ é um martingal. Sejam $0 \leq s<t$. Como $\mathbf{X}_{n}$ é um martingal, temos que

$$
\mathbf{E}\left(M\left(t \wedge \tau_{n}\right) \mathbb{1}_{\left[\tau_{n}>0\right]}|| \mathscr{F}_{s}\right)=M\left(s \wedge \tau_{n}\right) \mathbb{1}_{\left[\tau_{n}>0\right]}
$$

Por (3.25), a sequência $\left\{M^{2}\left(t \wedge \tau_{n}\right) \mathbb{1}_{\left[\tau_{n}>0\right]}\right\}_{n}$ é limitada em $\mathrm{L}^{2}$ e portanto* é uniformemente integrável. Além disso, para $n \rightarrow \infty, M\left(t \wedge \tau_{n}\right) \mathbb{1}_{\left[\tau_{n}>0\right]} \stackrel{\text { q.s }}{\longrightarrow} M(t)$ e portanto ${ }^{\dagger}$ temos também que $M\left(t \wedge \tau_{n}\right) \mathbb{1}_{\left[\tau_{n}>0\right]} \stackrel{\mathrm{L}^{1}}{\rightarrow} M(t)$. Assim, pelo teorema C.15 na página 163 e de (3.26), resulta

$$
\begin{aligned}
\lim _{n \rightarrow \infty} \mathbf{E}\left(M\left(t \wedge \tau_{n}\right) \mathbb{1}_{\left[\tau_{n}>0\right]}|| \mathscr{F}_{s}\right) & =\mathbf{E}\left(M(t) \| \mathscr{F}_{s}\right) \\
& =\lim _{n \rightarrow \infty} M\left(s \wedge \tau_{n}\right) \mathbb{1}_{\left[\tau_{n}>0\right]} \\
& =M_{s},
\end{aligned}
$$

como queríamos.

Finalmente, para provar que $\mathbf{M}^{2}-\llbracket \mathbf{M} \rrbracket$ é uniformemente integrável basta notar que

$$
\sup _{t \geq 0}\left|M^{2}(t)-\llbracket \mathbf{M} \rrbracket(t)\right| \leq \sup _{t \geq 0}|M(t)|^{2}+\lim _{t \rightarrow \infty} \llbracket \mathbf{M} \rrbracket(t)
$$

como $\sup _{t \geq 0}|M(t)|^{2}+\lim _{t \rightarrow \infty} \llbracket \mathbf{M} \rrbracket(t)$ é uma variável aleatória integrável, pela proposição B.9 na

\footnotetext{
* Veja o teorema B.8 na página 155. † Veja o corolário B.7 na página 155.
} 
página 156 - item vii, resulta que $\mathbf{M}^{2}-\llbracket \mathbf{M} \rrbracket$ é uniformemente integrável.

Corolário 3.22. Seja $\mathbf{M} \in c \mathcal{H}_{0}^{2}$ então $\|M\|_{\mathcal{H}^{2}}=\sqrt{\mathbf{E}\left(\llbracket \mathbf{M} \rrbracket_{\infty}\right)} \doteq\left\|\sqrt{\llbracket \mathbf{M} \rrbracket_{\infty}}\right\|_{2}$.

Demonstração. Na demonstração da proposição anterior, vimos que $\mathbf{E}\left(M_{\infty}^{2}\right)-\mathbf{E}\left(\llbracket \mathbf{M} \rrbracket_{\infty}\right)=\mathbf{E}\left(M_{0}^{2}\right)$. Como $M_{0}=0$, resulta que

$$
\|M\|_{2}^{\mathcal{H}^{2}}=\left\|M_{\infty}\right\|_{2}=\mathbf{E}\left(\llbracket \mathbf{M} \rrbracket_{\infty}\right)=\left\|\sqrt{\llbracket \mathbf{M} \rrbracket_{\infty}}\right\|_{2}^{2} .
$$

\subsection{Variação quadrática e semi-martingais}

Definição 3.23. Dizemos que um processo estocástico $\mathbf{X}$ é um semi-martingal contínuo quando admitir a seguinte representação

$$
X_{t}=M_{t}+A_{t}, t \geq 0
$$

onde $\mathbf{M}$ é um martingal local contínuo e $\mathbf{A}$ é um um processo contínuo, adaptado e de variação finita $\operatorname{com} A_{0}=0$.

O próximo resultado mostra que podemos nos referir a decomposição $X_{t}=M_{t}+A_{t}$ como a decomposição semi-martingal de $\mathbf{X}$.

Proposição 3.24. Seja $\mathbf{X}$ um semi-martingal contínuo. Então a decomposição $X_{t}=M_{t}+A_{t}$ é única.

Demonstração. Resulta do corolário 3.7. De fato, ele nos garante que existe um único processo contínuo de variação finita $\mathbf{A}$ com $A_{0}=0$ tal que $\mathbf{M}=\mathbf{X}-\mathbf{A}$ é um martingal local

Definição 3.25. Seja $\mathbf{X}$ um semi-martingal contínuo com decomposição $X_{t}=M_{t}+A_{t}$. Dizemos que o processo $\mathbf{M}$ é a parte martingal local de $\mathbf{X}$ e $\mathbf{A}$ é o processo compensador de $\mathbf{X}$. Em algumas ocasiões, usaremos a notação $u_{\mathbf{X}}$ para o processo compensador de $\mathbf{X}$.

Observamos que todo processo contínuo de variação finita $\mathbf{A}$ é um semi-martingal com parte martingal local $M_{t}=A_{0}$ e compensador $u_{\mathbf{A}}(t)=A_{t}-A_{0}$. 
Exemplo 3.26. Sejam $\mathbf{X}$ e $\mathbf{Y}$ martingais locais contínuos. Então $\mathbf{X}^{2}$ e $\mathbf{X Y}$ são semi-martingais contínuos. De fato, $\mathbf{X}^{2}=\left(\mathbf{X}^{2}-\llbracket \mathbf{X} \rrbracket\right)+\llbracket \mathbf{X} \rrbracket$ e $\mathbf{X Y}=(\mathbf{X Y}-\llbracket \mathbf{X}, \mathbf{Y} \rrbracket)+\llbracket \mathbf{X}, \mathbf{Y} \rrbracket$.

Definição 3.27. Sejam $\mathbf{X}=\mathbf{M}+\mathbf{A}$ e $\mathbf{Y}=\mathbf{N}+\mathbf{B}$ semi-martingais contínuos. Definimos o processo de covariação entre $\mathbf{X}$ e $\mathbf{Y}$ como

$$
\llbracket \mathbf{X}, \mathbf{Y} \rrbracket=\llbracket \mathbf{M}, \mathbf{N} \rrbracket \text {. }
$$

Note que se $\mathbf{A}$ é um processo contínuo de variação finita, então vimos que $\mathbf{A}$ é um semi-martingal contínuo com parte martingal local constante. Dessa forma, $\llbracket \mathbf{X}, \mathbf{A} \rrbracket=0$, para qualquer semimartingal contínuo X. O próximo resultado justifica a definição acima.

Proposição 3.28. Sejam $\mathbf{X}$ e $\mathbf{Y}$ semi-martingais contínuos e $\mathbf{A}$ um processo contínuo de variação finita. Então sendo $t \geq 0$ e $\mathcal{P}$ uma partição de $[0, t]$

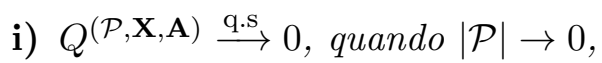

ii) $Q^{(\mathcal{P}, \mathbf{X}, \mathbf{Y})} \stackrel{\mathrm{P}}{\rightarrow} \llbracket \mathbf{X}, \mathbf{Y} \rrbracket_{t}$, quando $|\mathcal{P}| \rightarrow 0$.

Demonstração. i) Temos que existe um conjunto de medida nula $\Lambda$ tal que para $\omega \in \Lambda^{c}$, as trajétorias $s \mapsto A(s, \omega)$ são de variação finita e as trajétorias $s \mapsto X(s, \omega)$ são contínuas e portanto uniformemente contínuas no compacto $[0, t]$. Seja $\mathcal{P}: 0=t_{0}<t_{1}<\ldots<t_{n}=t$ uma partição de $[0, t]$. Temos que

$$
\begin{aligned}
\left|Q^{(\mathcal{P}, \mathbf{X}, \mathbf{A})}(\omega)\right| & \leq \sum_{j=1}^{n}\left|\left(X_{t_{j}}-X_{t_{j-1}}\right)(\omega)\right|\left|\left(A_{t_{j}}-A_{t_{j-1}}\right)(\omega)\right| \\
& \leq \sup _{1 \leq j \leq n}\left|\left(X_{t_{j}}-X_{t_{j-1}}\right)(\omega)\right| \sum_{j=1}^{n}\left|\left(A_{t_{j}}-A_{t_{j-1}}\right)(\omega)\right| .
\end{aligned}
$$

Da continuidade uniforme de $\mathbf{X}$ e finitude da variação de $\mathbf{A}$ em $\Lambda^{c}$, temos que para $|\mathcal{P}| \rightarrow 0$,

$$
\sup _{1 \leq j \leq n}\left|\left(X_{t_{j}}-X_{t_{j-1}}\right)(\omega)\right| \longrightarrow 0
$$

e também

$$
\sum_{j=1}^{n}\left|\left(A_{t_{j}}-A_{t_{j-1}}\right)(\omega)\right|<\infty .
$$


Donde segue o resultado.

ii) Sejam $\mathbf{X}=\mathbf{M}+\mathbf{B}$ e $\mathbf{Y}=\mathbf{N}+\mathbf{C}$ as decomposições dos semi-martingais $\mathbf{X}$ e $\mathbf{Y}$. Fixe $t \geq 0$ e seja $\mathcal{P}: 0=t_{0}<t_{1}<\ldots<t_{n}=t$ uma partição de $[0, t]$. Temos que

$$
\begin{aligned}
Q^{(\mathcal{P}, \mathbf{X}, \mathbf{Y})} & =\sum_{j=1}^{n}\left[\left(M_{t_{j}}-M_{t_{j-1}}\right)+\left(B_{t_{j}}-B_{t_{j-1}}\right)\right]\left[\left(N_{t_{j}}-N_{t_{j-1}}\right)+\left(C_{t_{j}}-C_{t_{j-1}}\right)\right] \\
& =Q^{(\mathcal{P}, \mathbf{B}, \mathbf{Y})}+Q^{(\mathcal{P}, \mathbf{M}, \mathbf{N})}+Q^{(\mathcal{P}, \mathbf{M}, \mathbf{C})} .
\end{aligned}
$$

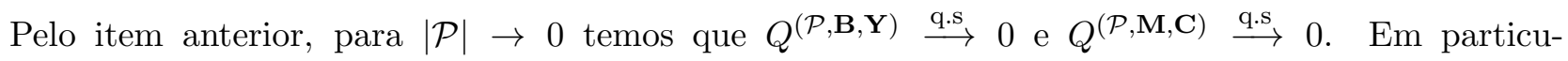
lar, essas convergências valem também em probabilidade. Agora, pela proposição 3.18 temos que $Q^{(\mathcal{P}, \mathbf{M}, \mathbf{N})} \stackrel{\mathrm{P}}{\rightarrow} \llbracket \mathbf{M}, \mathbf{N} \rrbracket_{t} \doteq \llbracket \mathbf{X}, \mathbf{Y} \rrbracket_{t}$, como queríamos.

Proposição 3.29. Sejam X, Y semi-martingais contínuos, $\tau$ um tempo de parada e $\xi$ uma variável aleatória $\mathscr{F}_{0}$-mensurável. Então

i) $\llbracket \mathbf{X}, \mathbf{Y} \rrbracket e \llbracket \mathbf{X} \rrbracket$ são processos contínuos, de variação finita e que se anulam em $t=0$.

ii) $\llbracket \mathbf{X} \rrbracket=0$ se, e somente se, $\mathbf{X}$ for um processo de variação finita.

iii) $\llbracket \mathbf{X}^{\tau}, \mathbf{Y}^{\tau} \rrbracket=\llbracket \mathbf{X}^{\tau}, \mathbf{Y} \rrbracket=\llbracket \mathbf{X}, \mathbf{Y} \rrbracket^{\tau}$.

iv) A covariação quadrática $\llbracket \cdot, \cdot \rrbracket$ é simetrica, bilinear e não negativa.

v) Sejam $\mathbf{A}$ e $\mathbf{B}$ processos contínuos e de variação finita então $\llbracket \mathbf{X}+\mathbf{A}, \mathbf{Y}+\mathbf{B} \rrbracket=\llbracket \mathbf{X}, \mathbf{Y} \rrbracket$.

vi) $\llbracket \xi \mathbf{X}, \mathbf{Y} \rrbracket=\xi \llbracket \mathbf{X}, \mathbf{Y} \rrbracket$.

Demonstração. i) Segue diretamente da definição 3.27, pois o resultado é válido para martingais locais.

ii) Seja $\mathbf{X}=\mathbf{M}+\mathbf{A}$ a decomposição de $\mathbf{X}$. Se $\llbracket \mathbf{X} \rrbracket=0$ então, por definição $\llbracket \mathbf{M} \rrbracket=0$ e pela proposição 3.13 - item iii), isso implica que $\mathbf{M}$ é constante. Logo, $\mathbf{X}$ é de variação finita pois é soma de um processo constante (em particular de variação finita) com um de variação finita. Reciprocamente, seja $\mathbf{X}$ um processo de variação finita, então $\mathbf{M}=\mathbf{X}-\mathbf{A}$ é de variação finita. Pelo teorema de Fisk* $\mathbf{M}$ é constante. Novamente pela proposição 3.13 - item iii), segue que $\llbracket \mathbf{M} \rrbracket=0$. Como, por definição, $\llbracket \mathbf{X} \rrbracket=\llbracket \mathbf{M} \rrbracket$ segue o resultado.

\footnotetext{
* Veja o teorema 3.6 na página 35
} 
iii) Como $\mathbf{X}^{\tau}=\mathbf{M}^{\tau}+\mathbf{A}^{\tau}$ e martingal local parado é martingal local ${ }^{*}$ temos que $\mathbf{X}^{\tau}$ é semimartingal. Assim $\llbracket \mathbf{X}^{\tau}, \mathbf{Y}^{\tau} \rrbracket=\llbracket \mathbf{M}^{\tau}, \mathbf{N}^{\tau} \rrbracket$ onde $\mathbf{M}$ e $\mathbf{N}$ são as partes martingais locais de $\mathbf{X}$ e $\mathbf{Y}$ respectivamente. Disso, o resultado segue da propriedade análoga para martingais locais.

iv) Segue pela definição da covariação para semi-martingais e da propriedade análoga para martingais locais.

v) Segue pela definição da covariação para semi-martingais.

vi) Seja $\mathbf{X}=\mathbf{M}+\mathbf{A}$ a decomposição de $\mathbf{X}$. Como $\xi$ é $\mathscr{F} 0$-mensurável temos que $\xi \mathbf{X}$ é adaptado. Sabemos $^{\dagger}$ que $\xi \mathbf{X}$ é martingal local e $\xi \mathbf{A}$ é um processo de variação finita. Assim, $\xi \mathbf{X}=\xi \mathbf{M}+\xi \mathbf{A}$ é um semi-martingal contínuo. Seja $t \geq 0$ e $\mathcal{P}$ uma partição arbitrária de $[0, t]$. Segue que $Q^{(\mathcal{P}, \xi \mathbf{X}, \mathbf{Y})}=$ $\xi Q^{(\mathcal{P}, \mathbf{X}, \mathbf{Y})}$ e fazendo $|\mathcal{P}| \rightarrow 0$ resulta que $\llbracket \xi \mathbf{X}, \mathbf{Y} \rrbracket_{t}=\xi \llbracket \mathbf{X}, \mathbf{Y} \rrbracket_{t}$.

\footnotetext{
* Veja a proposição 2.49 na página 28 - item iii). † Veja a proposição 2.49 na página 28 - item ii).
} 


\section{Capítulo 4}

\section{Integração estocástica}

I finally devised stochastic differential equations, after painstaking solitary endeavors.

Kiyoshi Itô

Considere dado um espaço de probabilidade filtrado. Uma estratégia de negociação é um processo estocástico adaptado $\mathbf{H}$ que determina para cada estado do mundo $\omega$ e instante de tempo $t$ a quantidade de uma ação que um investidor possui. Suponha que o preço dessa ação seja dado por um processo W. É claro que se a estratégia for constante durante um período de tempo, isto é, $H(t, \omega)=h, t \in[a, b], \forall \omega \in \Omega$, o ganho total (que pode ser negativo) do investidor no período é $h(W(b)-W(a))$. A teoria de integração estocástica se faz necessária para modelar os ganhos admitindo estratégias de negociação não triviais. Tal ganho será então denotado por $\int_{0}^{t} H_{s} d W_{s}$. Uma nova teoria de integração é necessária para interpretar esse símbolo, pois admitindo um modelo de mercado razoável espera-se que a maioria dos preços $\mathbf{W}$ dos ativos não sejam de variação finita (mais detalhes sobre isso em [7]).

\subsection{Integração estocástica com respeito a processos de variação finita}

Considere um espaço de probabilidade filtrado $\left(\Omega, \mathscr{F},\left\{\mathscr{F}_{t}\right\}, \mathrm{P}\right)$ completo e $\mathbf{A}$ um processo definido em $\mathbb{R}_{+}$cujas trajétorias são funções contínuas à direita e de variação finita. É um resultado clássico de análise (veja por exemplo [27]) que $A(\cdot, \omega)$ induz uma única medida de Borel com sinal $\mu_{\omega}^{t} \mathrm{em}$ 
cada intervalo $[0, t]$ de tal forma que

$$
\mu_{\omega}^{t}([a, b])=A(b, \omega)-A(a, \omega)
$$

para qualquer $[a, b] \subseteq[0, t] \mathrm{e}$

$$
\mu_{\omega}^{r}=\left.\mu_{\omega}^{t}\right|_{[0, r]},
$$

para todo $r \in(0, t]$. Dessa forma, vamos usar a notação $\mu_{\omega}^{\mathbf{A}}$. É usual também definir

$$
\int_{0}^{t} f(s) \mu_{\omega}^{\mathbf{A}}(d s) \doteq \int_{0}^{t} f(s) A(d s, \omega)
$$

onde $f$ é uma função integrável.

Vamos modificar um pouco a notação introduzida na definição 3.4 e considerar $t \mapsto \mathrm{V}_{\mathbf{A}}([0, t], \omega)$, a variação total de $\mathbf{A}$. Para simplificar mais ainda a notação, denotaremos $\mathrm{V}_{\mathbf{A}}([0, t], \omega) \doteq \mathrm{V}_{\mathbf{A}}(t, \omega)$. Temos que $\mathrm{V}_{\mathbf{A}}(\cdot, \omega)$ é uma função contínua à direita e não decrescente e portanto* induz uma única medida de Borel em $[0, \infty)$ denotada por $\left|\mu_{\omega}^{\mathbf{A}}\right|$, que satisfaz $\left|\mu_{\omega}^{\mathbf{A}}\right|([a, b])=\mathrm{V}_{\mathbf{A}}(b, \omega)-\mathrm{V}_{\mathbf{A}}(a, \omega)$, para qualquer $[a, b] \subseteq[0, \infty)$. Lembramos o leitor a notação usual

$$
\int_{0}^{t} f(s)\left|\mu_{\omega}^{\mathbf{A}}\right|(d s) \doteq \int_{0}^{t} f(s) \mathrm{V}_{\mathbf{A}}(d s, \omega) .
$$

quando a integral existir, que pode ser usada durante o texto. Observamos que a notação $\left|\mu_{\omega}^{\mathbf{A}}\right|$ não é uma coincidência. De fato, considere a decomposição de Hahn-Jordan de $\mu_{\omega}^{\mathbf{A}}$ :

$$
\mu_{\omega}^{\mathbf{A}}=\left(\mu_{\omega}^{\mathbf{A}}\right)^{+}-\left(\mu_{\omega}^{\mathbf{A}}\right)^{-}
$$

Temos que

$$
\left|\mu_{\omega}^{\mathbf{A}}\right|=\left(\mu_{\omega}^{\mathbf{A}}\right)^{+}+\left(\mu_{\omega}^{\mathbf{A}}\right)^{-}
$$

isto é, a medida $\left|\mu_{\omega}^{\mathbf{A}}\right|$ gerada pela variação de $\mathbf{A}$ é a variação total da medida $\mu_{\omega}^{\mathbf{A}}$, que foi gerada por A.

Teorema 4.1. Sejam $\mathbf{A}$ um processo estocástico definido em $\mathbb{R}_{+}$, adaptado à $\left\{\mathscr{F}_{t}\right\}$, contínuo à

\footnotetext{
* Para uma demonstração desse fato, veja [8].
} 


\subsection{INTEGRAÇÃO ESTOCÁSTICA COM RESPEITO A PROCESSOS DE VARIAÇÃO FINITA63}

direita e de variação finita, e $\mathbf{H}: \mathbb{R}_{+} \times \Omega \rightarrow \mathbb{R}$ um processo mensurável tal que para $\omega \in \Omega$ fixado,

$$
\int_{0}^{t}|H(s, \omega)| \mathrm{V}_{\mathbf{A}}(d s, \omega)<\infty, \quad \mathrm{P}-\mathrm{q} \text {. s. para cada } t \in \mathbb{R}_{+} .
$$

Então, para cada $t \in \mathbb{R}_{+}$fixado, $I(t, \cdot): \Omega \rightarrow \mathbb{R}$ definida como

$$
I(t, \omega) \doteq \int_{0}^{t} H(s, \omega) A(d s, \omega) \doteq(\mathbf{H} \bullet \mathbf{A})(t, \omega)
$$

é uma variável aleatória, isto é, uma função $\mathscr{F}$-mensurável. Além disso, se $\mathbf{H}$ for progressivamente mensurável então o processo $\left\{I_{t}: t \in \mathbb{R}_{+}\right\}$será $\left\{\mathscr{F}_{t}\right\}$-adaptado.

Demonstração. Inicialmente, note que $H(\cdot, \omega)$ é $\mu_{\omega}^{\mathbf{A}}$-integrável. De fato, como

$$
\int_{0}^{t}|H(s, \omega)|\left|\mu_{\omega}^{\mathbf{A}}\right|(d s)=\int_{0}^{t}|H(s, \omega)|\left(\mu_{\omega}^{\mathbf{A}}\right)^{+}(d s)+\int_{0}^{t}|H(s, \omega)|\left(\mu_{\omega}^{\mathbf{A}}\right)^{-}(d s)<\infty,
$$

temos que

$$
\int_{0}^{t}|H(s, \omega)|\left(\mu_{\omega}^{\mathbf{A}}\right)^{+}(d s)<\infty
$$

e

$$
\int_{0}^{t}|H(s, \omega)|\left(\mu_{\omega}^{\mathbf{A}}\right)^{-}(d s)<\infty
$$

portanto

$$
\int_{0}^{t}|H(s, \omega)| \mu_{\omega}^{\mathbf{A}}(d s)=\int_{0}^{t}|H(s, \omega)|\left(\mu_{\omega}^{\mathbf{A}}\right)^{+}(d s)-\int_{0}^{t}|H(s, \omega)|\left(\mu_{\omega}^{\mathbf{A}}\right)^{-}(d s)<\infty .
$$

Agora, vamos provar que $I(t, \cdot)$ é $\mathscr{F}$-mensurável. A decomposição

$$
A(t, \cdot)=\mathrm{V}_{\mathbf{A}}(t, \cdot)-\left(\mathrm{V}_{\mathbf{A}}(t, \cdot)-A(t, \cdot)\right)
$$


mostra que A é diferença de processos adaptados, crescentes e contínuos à direita. Assim, pela linearidade da integral de Lebesgue e como subtração de funções mensuráveis é mensuravel,

$$
\int_{0}^{t} H(s, \cdot) A(d s, \cdot)=\int_{0}^{t} H(s, \cdot) \mathrm{V}(d s, \cdot)-\int_{0}^{t} H(s, \cdot)(\mathrm{V}(d s, \cdot)-A(d s, \cdot))
$$

e portanto, podemos sem perda de generalidade, supor que $A(\cdot, \omega)$ é crescente e dessa forma as medidas $\mu_{\omega}$ associadas são positivas. Agora, pela decomposição $H(\cdot, \omega)=H^{+}(\cdot, \omega)-H^{-}(\cdot, \omega)$ é suficiente assumir que $H(\cdot, \omega)$ é não negativa. Como é usual na teoria de medida e integração, pelo fato de $\mathbf{H}$ ser $\sigma\left(\mathscr{B}_{\mathbb{R}_{+}} \times \mathscr{F}\right)$ - mensurável, $\mathbf{H}$ é o limite monótono de processos simples não negativos. Assim, pela linearidade da integral de Lebesgue e pelo teorema da convergência monótona, basta tratar o caso $\mathbf{H}=\mathbb{1}_{\Gamma}$, onde $\Gamma \subseteq \mathbb{R}_{+} \times \Omega$ é um conjunto $\sigma\left(\mathscr{B}_{\mathbb{R}_{+}} \times \mathscr{F}\right)$-mensurável. Agora, defina

$$
\mathcal{L} \doteq\left\{\Gamma \in \sigma\left(\mathscr{B}_{\mathbb{R}_{+}} \times \mathscr{F}\right): \int_{0}^{t} \mathbb{1}_{\Gamma}(s, \cdot) A(d s, \cdot) \in \mathscr{F}\right\}
$$

Devemos provar portanto que $\sigma\left(\mathscr{B}_{\mathbb{R}_{+}} \times \mathscr{F}\right) \subseteq \mathcal{L}$. Para isto, seja

$$
\mathcal{I} \doteq\{[a, b] \times F: 0 \leq a<b<\infty, F \in \mathscr{F}\}
$$

Temos que $\mathcal{I}$ é um $\pi$-sistema e $\mathcal{L}$ é um $\lambda$-sistema. Mas $\mathcal{I} \subseteq \mathcal{L}$ pois

$$
\int_{0}^{t} \mathbb{1}_{[a, b] \times F}(s, \cdot) A(d s, \cdot)=\mathbb{1}_{F}(\cdot)(A(t \wedge b, \cdot)-A(t \wedge a, \cdot)) \in \mathscr{F} .
$$

Como $\sigma(\mathcal{I})=\sigma\left(\mathscr{B}_{\mathbb{R}_{+}} \times \mathscr{F}\right)$, pelo teorema da classe monótona* resulta que $\sigma\left(\mathscr{B}_{\mathbb{R}_{+}} \times \mathscr{F}\right) \subseteq \mathcal{L}$.

Suponha agora que $\mathbf{H}$ é progressivamente mensurável e fixe $t \in \mathbb{R}_{+}$. Ao considerar $I(t, \cdot)=\int_{0}^{t} H(s, \cdot) A(d s, \cdot)$ usamos apenas a restrição $\left.\mathbf{H}\right|_{[0, t] \times \Omega}$ que é $\sigma\left(\mathscr{B}_{[0, t]} \times \mathscr{F}_{t}\right)$-mensurável. Logo, pelo mesmo argumento segue que $I(t, \cdot)$ é $\mathscr{F}_{t}$-mensurável e portanto $\left\{I_{t}: t \in \mathbb{R}_{+}\right\}$é $\left\{\mathscr{F}_{t}\right\}$ adaptado.

Definição 4.2. Seja $\mathbf{A}$ um processo contínuo e de variação finita. Definimos o espaço $\mathcal{L}_{\text {loc }}^{1}(\mathbf{A})$ como o conjunto de todos os processos $\mathbf{H}$ de trajetórias mensuráveis satisfazendo a relação (4.1).

\footnotetext{
* Veja o teorema A.2.
} 


\subsection{INTEGRAÇÃO ESTOCÁSTICA COM RESPEITO A PROCESSOS DE VARIAÇÃO FINITA65}

Definimos o conjunto $\mathcal{L}^{1}(\mathbf{A})$ como a família de todos os processos $\mathbf{H}$ mensuráveis satisfazendo

$$
\int_{0}^{\infty}|H(s, \omega)| \mathrm{V}_{A}(d s, \omega)<\infty, \quad \mathrm{P}-\mathrm{q} . \mathrm{s} .
$$

Definição 4.3. Seja $\tau$ um tempo de parada. Um intervalo estocástico $[0, \tau]$ deve ser entendido como o conjunto

$$
\left\{(t, \omega) \in \mathbb{R}_{+} \times \Omega: 0 \leq t \leq \tau(\omega)\right\} \subseteq \mathbb{R}_{+} \times \Omega
$$

Observamos que o processo $\mathbf{X}=\mathbb{1}_{[0, \tau]}$ é tal que $X(t, \omega)=\mathbb{1}_{[0, \tau(\omega)]}(t)$. Assim, note que multiplicar um processo por $\mathbb{1}_{[0, \tau]}$ tem o efeito de trunca-lo para 0 após $\tau$. Vejamos agora algumas propriedades da integral $\int_{0}^{t} H(s, \omega) A(d s, \omega)$.

Proposição 4.4. Sejam A um processo (contínuo) contínuo à direita e de variação finita, $\mathbf{H} \in$ $\mathcal{L}_{\text {loc }}^{1}(\mathbf{A})$ e $\tau$ um tempo de parada. Então*

i) $\mathbf{H} \bullet \mathbf{A}=\int_{0}^{\cdot} H(s, \cdot) A(d s, \cdot)$ é um processo (contínuo) contínuo à direita e de variação limitada.

ii) $\mathbf{H} \bullet \mathbf{A}$ é bilinear em $\mathbf{H}$ e $\mathbf{A}$.

iii) $\mathbf{H} \bullet\left(\mathbf{A}^{\tau}\right)=\left(\mathbb{1}_{[0, \tau]} \mathbf{H}\right) \bullet \mathbf{A}=(\mathbf{H} \bullet \mathbf{A})^{\tau}$.

* Veja a definição 2.18 na página 12. 
Demonstração. Para i, seja $t \geq 0$. Para $s_{1}, s_{2} \in[0, t], s_{1}<s_{2}$, temos

$$
\begin{aligned}
\left|\int_{0}^{s_{1}} H(s, \omega) \mu_{\omega}^{\mathbf{A}}(d s)-\int_{0}^{s_{2}} H(s, \omega) \mu_{\omega}^{\mathbf{A}}(d s)\right| & =\left|\int_{s_{1}}^{s_{2}} H(s, \omega) \mu_{\omega}^{\mathbf{A}}(d s)\right| \\
& =\left|\int_{s_{1}}^{s_{2}} H(s, \omega)\left(\mu_{\omega}^{\mathbf{A}}\right)^{+}(d s)-\int_{s_{1}}^{s_{2}} H(s, \omega)\left(\mu_{\omega}^{\mathbf{A}}\right)^{-}(d s)\right| \\
& \leq\left|\int_{s_{1}}^{s_{2}} H(s, \omega)\left(\mu_{\omega}^{\mathbf{A}}\right)^{+}(d s)\right|+\left|\int_{s_{1}}^{s_{2}} H(s, \omega)\left(\mu_{\omega}^{\mathbf{A}}\right)^{-}(d s)\right| \\
& \leq \int_{s_{1}}^{s_{2}}|H(s, \omega)|\left(\mu_{\omega}^{\mathbf{A}}\right)^{+}(d s)+\int_{s_{1}}^{s_{2}}|H(s, \omega)|\left(\mu_{\omega}^{\mathbf{A}}\right)^{-}(d s) \\
& =\int_{s_{1}}|H(s, \omega)|\left|\mu_{\omega}^{\mathbf{A}}\right|(d s) .
\end{aligned}
$$

Segue portanto que para qualquer $t \in \mathbb{R}_{+}$a variação de $\mathbf{H} \bullet \mathbf{A}$ é limitada por $\int_{0}^{t}|H(s, \omega)|\left|\mu_{\omega}^{\mathbf{A}}\right|(d s)$ que é finita por hipótese. A contínuidade à direita segue da mesma forma.

A demonstração de ii é a usual da teoria de integração.

Para iii, perceba que para cada $\omega \in \Omega$, a medida de Lebesgue-Stieltjes gerada pela trajetória $s \mapsto A^{\tau}(s, \omega)$ está concentrada no intervalo $(0, \tau(\omega)]$, assim o processo $\mathbf{H} \bullet \mathbf{A}^{\tau}$ permanece constante no intervalo $(\tau(\omega), \infty)$. O mesmo vale para os processos $\left(\mathbb{1}_{[0, \tau]} \mathbf{H}\right) \bullet \mathbf{A}$ e $(\mathbf{H} \bullet \mathbf{A})^{\tau}$. Como esses três processos são iguais no intervalo estocástico $[0, \tau]$, o resultado segue.

Uma outra propriedade é a associatividade. Por sua importância nesse trabalho, ela será enunciada separadamente. Antes, vamos precisar de dois resultados da teoria de medida e integração. Para o primeiro, o leitor pode encontrar uma demonstração por exemplo em [28].

Lema 4.5. Sejam $\mu$ e $\lambda$ medidas $\sigma$-finitas, com sinal, definidas em um espaço mensurável $(\Omega, \mathscr{F})$. Suponha que a derivada de Radon-Nikodym $\frac{d \lambda}{d \mu}$ de $\lambda$ com respeito a $\mu$ exista. Então, para toda função 


\subsection{INTEGRAÇÃO ESTOCÁSTICA COM RESPEITO A PROCESSOS DE VARIAÇÃO FINITA67}

$f: \mathbb{R} \rightarrow \overline{\mathbb{R}}, \mathscr{F}$-mensurável, temos que

$$
\int_{\Omega} f d \lambda=\int_{\Omega} f \frac{d \lambda}{d \mu} d \mu
$$

Lema 4.6. Considere o espaço mensurável $(\Omega, \mathscr{F})$ e $f: \Omega \rightarrow \overline{\mathbb{R}}$ uma função mensurável e $\mu$ uma medida com sinal em $(\Omega, \mathscr{F})$ tal que a integral

$$
\int_{\Omega} f d \mu
$$

exista. Considere $\nu$ uma medida com sinal em $(\Omega, \mathscr{F})$ definida como

$$
\nu(E)=\int_{E} f d \mu, \quad \forall E \in \mathscr{F} .
$$

Então a variação total de $\nu$ é tal que

$$
|\nu|(E)=\int_{E}|f| d|\mu|, \quad \forall E \in \mathscr{F} .
$$

Demonstração. Inicialmente, suponha que $\mu$ seja uma medida positiva. Defina $P_{f} \doteq\{\omega \in \Omega: f(\omega) \geq$ $0\}$ e $N_{f} \doteq\{\omega \in \Omega: f(\omega)<0\}$. Temos que $\left(P_{f}, N_{f}\right)$ é uma decomposição de Hahn para $\nu$. Dessa forma

$$
\nu^{+}(E)=\int_{E} f^{+} d \mu
$$

$\mathrm{e}$

$$
\nu^{-}(E)=\int_{E} f^{-} d \mu
$$

Assim

$$
|\nu|(E)=\nu^{+}(E)+\nu^{-}(E)=\int_{E}|f| d \mu .
$$


Considere agora $\mu$ medida com sinal. Note que

$$
\mu(E)=\int_{E} \phi d|\mu|
$$

onde $\phi: \Omega \rightarrow\{-1,1\}$ é uma função mensurável. Assim, pelo lema anterior, temos que

$$
\nu(E)=\int_{E} f d \mu=\int_{E} f \phi d|\mu|
$$

Finalmente, usando o resultado provado para medida positiva

$$
|\nu|(E)=\int_{E}|f \phi| d|\mu|=\int_{E}|f| d|\mu|
$$

como queríamos.

Teorema 4.7. Sejam A como na proposição 4.4, $\mathbf{K} \in \mathcal{L}_{\text {loc }}^{1}(\mathbf{A})$ e $\mathbf{H}$ um processo mensurável tal que para cada $\omega \in \Omega$,

$$
\int_{0}^{t}|H(s, \omega)| \mathrm{V}_{\mathbf{K} \bullet \mathbf{A}}(d s, \omega)<\infty, \quad \mathrm{P}-\mathrm{q} . \mathrm{s} . \text { para cada } t \in \mathbb{R}_{+},
$$

isto é, $\mathbf{H} \in \mathcal{L}_{\text {loc }}^{1}(\mathbf{K} \bullet \mathbf{A})$. Então $\mathbf{H K} \in \mathcal{L}_{\text {loc }}^{1}(\mathbf{A})$ e vale que

$$
\mathbf{H} \bullet(\mathbf{K} \bullet \mathbf{A})=(\mathbf{H K}) \bullet \mathbf{A} .
$$

Demonstração. Seja $\omega \in \Omega$. Para cada $t \geq 0$, considere a função

$$
\left(|\mathbf{K}| \bullet \mathrm{V}_{\mathbf{A}}\right)(t, \omega)=\int_{0}^{t}|K(s, \omega)|\left|\mu_{\omega}^{\mathbf{A}}\right|(d s) .
$$

Como $|\mathbf{K}| \bullet \mathrm{V}_{\mathbf{A}}$ é contínua à direita e de variação finita, ela gera uma medida que vamos denotar por $\mu^{|\mathbf{K}| \bullet V_{\mathbf{A}}}$ de tal forma que

$$
\mu_{\omega}^{|\mathbf{K}| \bullet V_{\mathbf{A}}}(E)=\int_{E}|K(s, \omega)|\left|\mu_{\omega}^{\mathbf{A}}\right|(d s)
$$




\subsection{INTEGRAÇÃO ESTOCÁSTICA COM RESPEITO A PROCESSOS DE VARIAÇÃO FINITA69}

para qualquer boreleano $E$. Dessa forma

$$
|K(\cdot, \omega)|=\frac{d \mu_{\omega}^{|\mathbf{K}| \bullet \mathrm{V}_{\mathbf{A}}}}{d\left|\mu_{\omega}^{\mathbf{A}}\right|}
$$

e pelo lema 4.5 ,

$$
\int_{0}^{t}|H K(s, \omega)|\left|\mu_{\omega}^{\mathbf{A}}\right|(d s)=\int_{0}^{t}|H(s, \omega)| \mu_{\omega}^{|\mathbf{K}| \bullet \mathbf{V}_{\mathbf{A}}}(d s) .
$$

Porém, note que

$$
\mu_{\omega}^{\mathbf{K} \bullet \mathbf{A}}(E)=\int_{E} K(s, \omega) \mu_{\omega}^{\mathbf{A}}(d s)
$$

e, pelo lema anterior segue que

$$
\left|\mu_{\omega}^{\mathbf{K} \bullet \mathbf{A}}\right|(E)=\int_{E}|K(s, \omega)|\left|\mu_{\omega}^{\mathbf{A}}\right|(d s) .
$$

Portanto, de (4.2) e (4.4) temos que $\left|\mu_{\omega}^{\mathrm{K} \bullet \mathbf{A}}\right|=\mu_{\omega}^{|\mathbf{K}| \bullet \boldsymbol{V} \mathbf{A}}$, usando isso, de (4.3) ficamos com

$$
\begin{aligned}
\int_{0}^{t}|H K(s, \omega)|\left|\mu_{\omega}^{\mathbf{A}}\right|(d s) & =\int_{0}^{t}|H(s, \omega)|\left|\mu_{\omega}^{\mathbf{K} \bullet \mathbf{A}}\right|(d s) \\
& \doteq \int_{0}^{t}|H(s, \omega)| \mathbf{V}_{\mathbf{K} \bullet \mathbf{A}}(d s, \omega) \\
& <\infty .
\end{aligned}
$$

E assim, provamos que HK satisfaz (4.1).

Finalmente, note que para qualquer $E$ boreleano,

$$
\mu_{\omega}^{\mathbf{K} \bullet \mathbf{A}}(E)=\int_{E} K(s, \omega) \mu_{\omega}^{\mathbf{A}}(d s),
$$

e assim,

$$
K(\cdot, \omega)=\frac{d \mu_{\omega}^{\mathbf{K} \bullet \mathbf{A}}}{d \mu_{\omega}^{\mathbf{A}}}
$$


Novamente pelo lema 4.5 resulta

$$
\begin{aligned}
\mathbf{H} \bullet(\mathbf{K} \bullet \mathbf{A}) & \doteq \int_{0}^{t} H(s, \omega) \mu_{\omega}^{\mathbf{K} \bullet \mathbf{A}}(d s) \\
& =\int_{0}^{t}(H K(s, \omega)) \mu_{\omega}^{\mathbf{A}}(d s) \\
& \doteq(\mathbf{H K}) \bullet \mathbf{A} .
\end{aligned}
$$

que é a relação desejada.

\subsection{A desigualdade de Kunita e Watanabe}

Vamos unir os resultados sobre variação quadrática e integração estocástica com respeito à processos de variação finita e provar um resultado crucial para a teoria de integração estocástica. Nesta seção $\mathbf{M}$ e $\mathbf{N}$ são martingais locais contínuos e $t>0$. Como sabemos, podemos considerar $\llbracket \mathbf{M}, \mathbf{N} \rrbracket$ como um processo contínuo e de variação finita e $\llbracket \mathbf{M} \rrbracket$ e $\llbracket \mathbf{N} \rrbracket$ processos contínuos e crescentes (e portanto também de variação finita). Denotaremos também por $\mu_{\omega}^{\llbracket \mathbf{M}, \mathbf{N} \rrbracket}, \mu_{\omega}^{\llbracket \mathbf{M} \rrbracket}, \mu_{\omega}^{\llbracket \mathbf{N} \rrbracket}$ e $\left|\mu_{\omega}^{\llbracket \mathbf{M}, \mathbf{N} \rrbracket}\right|$ as medidas com sinal de Lebesgue-Stieltjes geradas, respectivamente, pelas trajetórias dos processos $\llbracket \mathbf{M}, \mathbf{N} \rrbracket, \llbracket \mathbf{M} \rrbracket, \llbracket \mathbf{N} \rrbracket$ e $\mathrm{V}_{\llbracket \mathbf{M}, \mathbf{N} \rrbracket}$. Para $a<b$, usaremos também a notação

$$
\llbracket \mathbf{M} \rrbracket_{a}^{b} \doteq \llbracket \mathbf{M} \rrbracket_{b}-\llbracket \mathbf{M} \rrbracket_{a}
$$

Pelo teorema 3.12, vale a representação

$$
\llbracket \mathbf{M} \rrbracket_{a}^{b}=\lim _{|\mathcal{P}| \rightarrow 0} Q^{(\mathcal{P}, \mathbf{M})} \text { em probabilidade }
$$

onde o limite é tomado sob todas as partições $\mathcal{P}$ do intervalo $[a, b]$.

Lema 4.8. Para qualquer sub intervalo $[a, b] \subseteq[0, t]$ temos para quase todo $\omega \in \Omega$,

$$
\left|\mu_{\omega}^{\llbracket \mathbf{M}, \mathbf{N} \rrbracket}\right|([a, b]) \leq\left(\mu_{\omega}^{\llbracket \mathbf{M} \rrbracket}([a, b])\right)^{\frac{1}{2}}\left(\mu_{\omega}^{\llbracket \mathbf{N} \rrbracket}([a, b])\right)^{\frac{1}{2}} .
$$

Demonstração. O plano será o seguinte: Fixado $[a, b] \subseteq[0, t]$ vamos provar que existe um conjunto 
$\Lambda$, independente da escolha de $a$ e $b$, tal que $\mathrm{P}(\Lambda)=0$ e para $\omega \in \Lambda^{c}$ vale que*

$$
\left.\left|\mu_{\omega}^{\llbracket \mathbf{M}, \mathbf{N} \rrbracket}([a, b])\right| \leq\left(\mu_{\omega}^{\llbracket \mathbf{M} \rrbracket}([a, b])\right)^{\frac{1}{2}}\left(\mu_{\omega}^{\llbracket \mathbf{N} \rrbracket}\right)([a, b])\right)^{\frac{1}{2}} .
$$

Desta forma, se $\mathcal{P}: a=t_{0}<t_{1}<\ldots<t_{n}=b$ é uma partição de $[a, b]$, então para qualquer $\omega \in \Lambda^{c}$ e $j=1,2, \ldots, n$,

$$
\left|\mu_{\omega}^{\llbracket \mathbf{M}, \mathbf{N} \rrbracket}\left(\left[t_{j-1}, t_{j}\right]\right)\right| \leq\left(\mu_{\omega}^{\llbracket \mathbf{M} \rrbracket}\left(\left[t_{j-1}, t_{j}\right]\right)\right)^{\frac{1}{2}}\left(\mu_{\omega}^{\llbracket \mathbf{N} \rrbracket}\left(\left[t_{j-1}, t_{j}\right]\right)\right)^{\frac{1}{2}} .
$$

Somando e usando a desigualdade de Cauchy-Schwarz:

$$
\begin{aligned}
\sum_{j=1}^{n}\left|\mu_{\omega}^{\llbracket \mathbf{M}, \mathbf{N} \rrbracket}\left(\left[t_{j-1}, t_{j}\right]\right)\right| & \leq\left(\sum_{j=1}^{n} \mu_{\omega}^{\llbracket \mathbf{M} \rrbracket}\left(\left[t_{j-1}, t_{j}\right]\right)\right)^{\frac{1}{2}}\left(\sum_{j=1}^{n} \mu_{\omega}^{\llbracket \mathbf{N} \rrbracket}\left(\left[t_{j-1}, t_{j}\right]\right)\right)^{\frac{1}{2}} \\
& \left.=\left(\mu_{\omega}^{\llbracket \mathbf{M} \rrbracket}([a, b])\right)^{\frac{1}{2}}\left(\mu_{\omega}^{\llbracket \mathbf{N} \rrbracket}\right)([a, b])\right)^{\frac{1}{2}} .
\end{aligned}
$$

Tomando o sup sob todas as partições de $[a, b]$ resulta (4.5).

Agora vamos executar o plano. Para cada $r \in \mathbb{N}$, sejam $a_{r}, b_{r} \in \mathbb{Q}$ tais que $0 \leq a_{r}<b_{r} \leq t$ e $\left\{\mathcal{P}_{n}\right\}_{n}$ uma sequência de partições de $\left[a_{r}, b_{r}\right]$ tal que $\lim _{n \rightarrow \infty}\left|\mathcal{P}_{n}\right|=0$. Temos ${ }^{\dagger}$,

$$
Q^{\left(\mathcal{P}_{n}, \mathbf{N}, \mathbf{M}\right)} \stackrel{\mathrm{P}}{\rightarrow} \llbracket \mathbf{M}, \mathbf{N} \rrbracket_{a_{r}}^{b_{r}}, \quad Q^{\left(\mathcal{P}_{n}, \mathbf{M}\right)} \stackrel{\mathrm{P}}{\rightarrow} \llbracket \mathbf{M} \rrbracket_{a_{r}}^{b_{r}}, \quad \text { e } Q^{\left(\mathcal{P}_{n}, \mathbf{N}\right)} \stackrel{\mathrm{P}}{\rightarrow} \llbracket \mathbf{N}_{a_{r}}^{b_{r}} .
$$

Mas a convergência em probabilidade garante a existência de uma subsequência de partições $\left\{\mathcal{P}_{n_{k}}\right\}_{k}$, onde a convergência é quase certa. Isto é, existe um conjunto de medida nula $\Lambda_{a_{r}, b_{r}}$ tal que se $\omega \in \Lambda_{a_{r}, b_{r}}^{c}$

$$
Q^{\left(\mathcal{P}_{n_{k}}, \mathbf{N}, \mathbf{M}\right)} \longrightarrow \llbracket \mathbf{M}, \mathbf{N} \rrbracket_{a_{r}}^{b_{r}}, \quad Q^{\left(\mathcal{P}_{n_{k}}, \mathbf{M}\right)} \longrightarrow \llbracket \mathbf{M} \rrbracket_{a_{r}}^{b_{r}}, \text { e } Q^{\left(\mathcal{P}_{n_{k}}, \mathbf{N}\right)} \longrightarrow \llbracket \mathbf{N} \rrbracket_{a_{r}}^{b_{r}}
$$

Defina agora $\Lambda \doteq \bigcup_{r \in \mathbb{N}} \Lambda_{a_{r}, b_{r}}$. Temos que $\mathrm{P}(\Lambda)=0$ e para $\omega \in \Lambda^{c}$ e qualquer $r \in \mathbb{N}$,

$$
Q^{\left(\mathcal{P}_{n_{k}}, \mathbf{N}, \mathbf{M}\right)} \longrightarrow \llbracket \mathbf{M}, \mathbf{N} \rrbracket_{a_{r}}^{b_{r}}, \quad Q^{\left(\mathcal{P}_{n_{k}}, \mathbf{M}\right)} \longrightarrow \llbracket \mathbf{M} \rrbracket_{a_{r}}^{b_{r}}, \text { e } Q^{\left(\mathcal{P}_{n_{k}}, \mathbf{N}\right)} \longrightarrow \llbracket \mathbf{N} \rrbracket_{a_{r}}^{b_{r}}
$$

Ou seja, $\Lambda$ definido dessa forma, não depende da escolha de $a_{r}$ e nem de $b_{r}$. Agora sejam $[a, b] \subseteq[0, t]$ * Alertamos o leitor com a notação: $\left|\mu_{\omega}^{\llbracket \mathbb{M}, \mathbf{N} \rrbracket}([a, b])\right| \neq\left|\mu_{\omega}^{\llbracket \mathrm{M}, \mathbf{N} \rrbracket}\right|([a, b]) ! \quad \dagger$ Veja a notação da definição 3.8 na página 37. 
arbitrário, $\omega \in \Lambda^{c},\left\{\mathcal{P}_{n}\right\}_{n}$ uma sequência de partições de $[a, b]$ e $\left\{a_{r}\right\}$ e $\left\{b_{r}\right\}$ sequências racionais tais que $\lim _{r \rightarrow \infty} a_{r}=a$ e $\lim _{r \rightarrow \infty} b_{r}=b$. Temos pela desigualdade de Cauchy-Schwarz

$$
\begin{aligned}
\left|Q^{\left(\mathcal{P}_{n}, \mathbf{N}, \mathbf{M}\right)}(\omega)\right| & =\left|\sum_{i=1}^{n}\left[M_{t_{j}}-M_{t_{j-1}}\right]\left[N_{t_{j}}-N_{t_{j-1}}\right](\omega)\right|^{2} \\
& \leq \sum_{i=1}^{n}\left[\left(M_{t_{j}}-M_{t_{j-1}}\right)(\omega)\right]^{2} \sum_{i=1}^{n}\left[\left(N_{t_{j}}-N_{t_{j-1}}\right)(\omega)\right]^{2} \\
& =Q^{\left(\mathcal{P}_{n}, \mathbf{M}\right)}(\omega) Q^{\left(\mathcal{P}_{n}, \mathbf{N}\right)}(\omega) .
\end{aligned}
$$

Tomando as raizes quadradas, e fazendo $n \rightarrow \infty$ obtemos

$$
\left|\llbracket \mathbf{M}, \mathbf{N} \rrbracket_{a_{r}}^{b_{r}}(\omega)\right| \leq\left(\llbracket \mathbf{M} \rrbracket_{a_{r}}^{b_{r}}(\omega)\right)^{\frac{1}{2}}\left(\llbracket \mathbf{N} \rrbracket_{a_{r}}^{b_{r}}(\omega)\right)^{\frac{1}{2}} .
$$

Finalmente, fazendo $r \rightarrow \infty$

$$
\left|\llbracket \mathbf{M}, \mathbf{N} \rrbracket_{a}^{b}(\omega)\right| \leq\left(\llbracket \mathbf{M} \rrbracket_{a}^{b}(\omega)\right)^{\frac{1}{2}}\left(\llbracket \mathbf{N} \rrbracket_{a}^{b}(\omega)\right)^{\frac{1}{2}}
$$

o que é equivalente a (4.6).

Vamos agora estender o domínio desigualdade anterior para todos os boreleanos de $[0, t]$. Antes vamos enunciar um resultado de medida que será útil.

Lema 4.9. Seja $(\Omega, \mathscr{F}, \mathrm{P})$ um espaço de probabilidade e $\mathcal{A} \subseteq \mathscr{F}$ uma álgebra de conjuntos tais que $\sigma(\mathcal{A})=\mathscr{F}$. Então para cada $E \in \mathscr{F}$ e $\varepsilon>0$, existe um conjunto $A \in \mathcal{A}$ tal que $\mathrm{P}(A \Delta E)<\varepsilon$, onde $A \Delta E \doteq(A-E) \cup(E-A)$.

Lema 4.10. Para qualquer $A \in \mathscr{B}_{[0, t]}$ temos para quase todo $\omega \in \Omega$,

$$
\left|\mu_{\omega}^{\llbracket \mathbf{M}, \mathbf{N} \rrbracket}\right|(A) \leq\left(\mu_{\omega}^{\llbracket \mathrm{M} \rrbracket}(A)\right)^{\frac{1}{2}}\left(\mu_{\omega}^{\llbracket \mathbf{N} \rrbracket}(A)\right)^{\frac{1}{2}} .
$$

Demonstração. Como as medidas se anulam nos conjuntos unitários, é suficiente provar a afirmação para $A \in \mathscr{B}_{[0, t)}$. Agora seja $\omega \in \Omega$ tal que (4.8) vale para qualquer intervalo $[a, b] \subseteq[0, t]$ e considere a família $\mathcal{G}$ de subconjuntos formados por uniões disjuntas de intervalos da forma $[a, b) \subseteq[0, t)$. Temos que $\mathcal{G}$ é uma álgebra e $\sigma(\mathcal{G})=\mathscr{B}_{[0, t)}$. Pelo lema anterior, se $A \in \mathscr{B}_{([0, t)}$ então existe uma sequência 
$\left\{A_{n}\right\} \in \mathcal{G}$ tal que

$$
\left(\left|\mu_{\omega}^{\llbracket \mathbf{M}, \mathbf{N} \rrbracket}\right|+\mu_{\omega}^{\llbracket \mathbf{M} \rrbracket}+\mu_{\omega}^{\llbracket \mathbf{N} \rrbracket}\right)\left(A \Delta A_{n}\right) \longrightarrow 0
$$

e portanto, quando $n \rightarrow \infty$,

$$
\left|\mu_{\omega}^{\llbracket \mathbf{M}, \mathbf{N} \rrbracket}\right|\left(A_{n}\right) \longrightarrow\left|\mu_{\omega}^{\llbracket \mathbf{M}, \mathbf{N} \rrbracket}\right|(A), \mu_{\omega}^{\llbracket \mathbf{M} \rrbracket}\left(A_{n}\right) \longrightarrow \mu_{\omega}^{\llbracket \mathbf{M} \rrbracket}(A) \text { e } \mu_{\omega}^{\llbracket \mathbf{N} \rrbracket}\left(A_{n}\right) \longrightarrow \mu_{\omega}^{\llbracket \mathbf{N} \rrbracket}(A)
$$

Dessa forma, basta provar o resultado para conjuntos em $\mathcal{G}$. Seja $A=\left[a_{1}, b_{1}\right) \cup\left[a_{2}, b_{2}\right) \cup \ldots \cup\left[a_{n}, b_{n}\right)$ com $0 \leq a_{1}<b_{1} \leq a_{2}<b_{2} \leq \cdots \leq a_{n}<b_{n}$. Pelo lema 4.8 e pela desigualdade de Cauchy-Schwarz resulta

$$
\begin{aligned}
\left|\mu_{\omega}^{\llbracket \mathbf{M}, \mathbf{N} \rrbracket}\right|(A) & =\sum_{j=1}^{n}\left|\mu_{\omega}^{\llbracket \mathbf{M}, \mathbf{N} \rrbracket}\right|\left(\left[a_{j}, b_{j}\right)\right) \\
& \leq \sum_{j=1}^{n}\left(\mu_{\omega}^{\llbracket \mathbf{M} \rrbracket}\left(\left[a_{j}, b_{j}\right)\right)\right)^{\frac{1}{2}}\left(\mu_{\omega}^{\llbracket \mathbf{N} \rrbracket}\left(\left[a_{j}, b_{j}\right)\right)\right)^{\frac{1}{2}} \\
& \leq\left[\sum_{j=1}^{n} \mu_{\omega}^{\llbracket \mathbf{M} \rrbracket}\left(\left[a_{j}, b_{j}\right)\right)\right]^{\frac{1}{2}}\left[\sum_{j=1}^{n} \mu_{\omega}^{\llbracket \mathbf{N} \rrbracket}\left(\left[a_{j}, b_{j}\right)\right)\right]^{\frac{1}{2}} \\
& =\left(\mu_{\omega}^{\llbracket \mathbf{M} \rrbracket}(A)\right)^{\frac{1}{2}}\left(\mu_{\omega}^{\llbracket \mathbf{N} \rrbracket}(A)\right)^{\frac{1}{2}},
\end{aligned}
$$

como queríamos.

Lema 4.11. Sejam $f, g:[0, t] \rightarrow \overline{\mathbb{R}}$ funções Borel-mensuráveis. Então

$$
\int_{0}^{t} f g d\left|\mu_{\omega}^{\llbracket \mathbf{M}, \mathbf{N} \rrbracket}\right| \leq\left(\int_{0}^{t} f^{2} d \mu_{\omega}^{\llbracket \mathbf{M} \rrbracket}\right)^{\frac{1}{2}}\left(\int_{0}^{t} g^{2} d \mu_{\omega}^{\llbracket \mathbf{N} \rrbracket}\right)^{\frac{1}{2}}
$$

para quase todo $\omega \in \Omega$.

Demonstração. Seja $\omega \in \Omega$ tal que (4.8) esteja satisfeito. Como é usual na teoria de medida e integração, basta provar o resultado para funções simples $f$ e $g$

$$
f=\sum_{j=1}^{n} \alpha_{j} \mathbb{1}_{A_{j}} \quad \text { e } \quad g=\sum_{j=1}^{n} \beta_{j} \mathbb{1}_{A_{j}}
$$


onde $\left\{A_{j}\right\}, j=1,2, \ldots, n$ é uma partição de $[0, t] \operatorname{com} A_{j} \in \mathscr{B}$ para cada $j$. Dessa forma, como $\left\{A_{j}\right\}$ é uma sequência disjunta temos que

$$
f^{2}=\sum_{j=1}^{n} \alpha_{j}^{2} \mathbb{1}_{A_{j}}, \quad g^{2}=\sum_{j=1}^{n} \beta_{j}^{2} \mathbb{1}_{A_{j}} \quad \text { e } \quad f g=\sum_{j=1}^{n} \alpha_{j} \beta_{j} \mathbb{1}_{A_{j}} .
$$

Assim, pelo lema (4.10) e pela desigualdade de Cauchy-Schwarz

$$
\begin{aligned}
\int_{0}^{t} f g d\left|\mu_{\omega}^{\llbracket \mathbf{M}, \mathbf{N} \rrbracket}\right| & =\sum_{j=1}^{n} \alpha_{j} \beta_{j}\left|\mu_{\omega}^{\llbracket \mathbf{M}, \mathbf{N} \rrbracket}\right|\left(A_{j}\right) \\
& \leq\left[\sum_{j=1}^{n} \alpha_{j}^{2} \mu_{\omega}^{\llbracket \mathbf{M} \rrbracket}\left(A_{j}\right)\right]^{\frac{1}{2}}\left[\sum_{j=1}^{n} \beta_{j}^{2} \mu_{\omega}^{\llbracket \mathbf{N} \rrbracket}\left(A_{j}\right)\right]^{\frac{1}{2}} \\
& =\left(\int_{0}^{t} f^{2} d \mu_{\omega}^{\llbracket \mathbf{M} \rrbracket}\right)^{\frac{1}{2}}\left(\int_{0}^{t} g^{2} d \mu_{\omega}^{\llbracket \mathbf{N} \rrbracket}\right)^{\frac{1}{2}}
\end{aligned}
$$

como queríamos.

Dos resultados anteriores, provamos o

Teorema 4.12. Sejam M, N martingais locais contínuos, $\mathbf{U}$ e $\mathbf{H}$ processos mensuráveis. Então para $t \geq 0$ vale a desigualdade de Kunita-Watanabe

$$
\int_{0}^{t}|U H(s, \omega)| \mathrm{V}_{\llbracket \mathbf{M}, \mathbf{N} \rrbracket}(d s, \omega) \leq\left(\int_{0}^{t} U^{2}(s, \omega) \llbracket \mathbf{M} \rrbracket(d s, \omega)\right)^{\frac{1}{2}}\left(\int_{0}^{t} H^{2}(s, \omega) \llbracket \mathbf{N} \rrbracket(d s, \omega)\right)^{\frac{1}{2}},
$$

para quase todo $\omega \in \Omega$.

\subsection{Integração estocástica com respeito a semi-martingais}

Sejam $\Gamma \in \sigma\left(\mathscr{B}_{\mathbb{R}_{+}} \times \mathscr{F}\right)$ e $\mathbf{M}$ um martingal local contínuo e considere a versão càdlàg e crescente da variação quadrática $\llbracket \mathbf{M} \rrbracket$. Temos* para cada $n \geq 1$ que $\int_{0}^{n} \mathbb{1}_{\Gamma}(s, \cdot) \llbracket \mathbf{M} \rrbracket(d s, \cdot)$ é $\mathscr{F}$ mensurável. Além disso, como $\int_{0}^{n} \mathbb{1}_{\Gamma}(s, \cdot) \llbracket \mathbf{M} \rrbracket(d s, \cdot) \nearrow \int_{0}^{\infty} \mathbb{1}_{\Gamma}(s, \cdot) \llbracket \mathbf{M} \rrbracket(d s, \cdot)$, temos pelo teorema

\footnotetext{
* Veja o teorema 4.1 na página 62.
} 
da convergência monótona que $\int_{0}^{\infty} \mathbb{1}_{\Gamma}(s, \cdot) \llbracket \mathbf{M} \rrbracket(d s, \cdot)$ é $\mathscr{F}$-mensurável e

$$
\mathbf{E}\left(\lim _{n \rightarrow \infty} \int_{0}^{n} \mathbb{1}_{\Gamma}(s, \cdot) \llbracket \mathbf{M} \rrbracket(d s, \cdot)\right)=\mathbf{E}\left(\int_{0}^{\infty} \mathbb{1}_{\Gamma}(s, \cdot) \llbracket \mathbf{M} \rrbracket(d s, \cdot)\right) .
$$

Motivados por essa discussão temos a seguinte

Definição 4.13. Definimos a medida de Dóleans gerada pela variação quadrática do martingal local contínuo $\mathbf{M}$ como a medida $\alpha_{\mathbf{M}}$ em $\sigma\left(\mathscr{B}_{\mathbb{R}_{+}} \times \mathscr{F}\right)$ dada por

$$
\alpha_{\mathbf{M}}(\Gamma) \doteq \mathbf{E}\left(\int_{0}^{\infty} \mathbb{1}_{\Gamma} d \llbracket \mathbf{M} \rrbracket\right)
$$

Definição 4.14. Seja $\mathbf{M}$ um martingal local contínuo. Denotaremos por $\mathcal{L}^{2}(\mathbf{M})$ o espaço das classes de equivalências de funções de quadrado integrável e progressivamente mensuráveis definidas no espaço de medida $\left(\mathbb{R}_{+} \times \Omega, \mathfrak{P}_{g}, \alpha_{\mathbf{M}}\right)$ isto é, $\mathcal{L}^{2}(\mathbf{M})=\mathrm{L}^{2}\left(\mathbb{R}_{+} \times \Omega, \mathfrak{P}_{g}, \alpha_{\mathbf{M}}\right)$. Denotaremos por $\|\cdot\|_{\mathbf{M}}$ a norma no espaço de Hilbert $\mathcal{L}^{2}(\mathbf{M})$

$$
\|\mathbf{X}\|_{\mathbf{M}} \doteq \sqrt{\int_{\mathbb{R}_{+} \times \Omega} \mathbf{X}^{2} d \alpha_{\mathbf{M}}}=\sqrt{\mathbf{E}\left(\int_{0}^{\infty} X^{2} d \llbracket \mathbf{M} \rrbracket\right)} .
$$

Proposição 4.15. Seja $\tau$ um tempo de parada. Então $\|\mathbf{H}\|_{\mathbf{M}^{\tau}}^{2}=\left\|\mathbf{H} \mathbb{1}_{[[0, \tau]]}\right\|_{\mathbf{M}}^{2}$, ou seja $\mathbf{H} \in \mathcal{L}^{2}\left(\mathbf{M}^{\tau}\right)$ se e somente se $\mathbf{H}_{[[0, \tau]]} \in \mathcal{L}^{2}(\mathbf{M})$ para cada processo progresivamente mensurável $\mathbf{H}$.

Demonstração. De fato, pela proposição 4.4 - item iii) temos para todo $t \geq 0$, $\left(\mathbf{H}^{2} \bullet \llbracket \mathbf{M}^{\tau} \rrbracket\right)_{t}=\left(\left(\mathbf{H}^{2} \mathbb{1}_{[[0, \tau]]}\right) \bullet \llbracket \mathbf{M} \rrbracket\right)_{t}$. Assim, fazendo $t \rightarrow \infty$

$$
\int_{0}^{\infty} H^{2}(s, \omega) \llbracket \mathbf{M}^{\tau} \rrbracket(d s, \omega)=\int_{0}^{\infty} H^{2} \mathbb{1}_{[[0, \tau]]}(s, \omega) \llbracket \mathbf{M} \rrbracket(d s, \omega)=\int_{0}^{\tau(\omega)} H^{2}(s, \omega) \llbracket \mathbf{M} \rrbracket(d s, \omega) .
$$

Lema 4.16. Sejam $\mathbf{M}$ um martingal local contínuo e $\mathbf{H} \in \mathcal{L}^{2}(\mathbf{M})$. Então para qualquer $\mathbf{N} \in c \mathcal{H}^{2}$, 
temos que $\mathbf{H} \in \mathcal{L}^{1}(\llbracket \mathbf{M}, \mathbf{N} \rrbracket)$, isto é,

$$
\int_{0}^{\infty}|H(s, \omega)|\left|\mu_{\omega}^{\llbracket \mathbf{M}, \mathbf{N} \rrbracket}\right|(d s)<\infty
$$

e o processo $\mathbf{H} \bullet \llbracket \mathbf{M}, \mathbf{N} \rrbracket$ está bem definido, é contínuo e de variação limitada.

Demonstração. Pela proposição 3.16 podemos supor que $\llbracket \mathbf{M}, \mathbf{N} \rrbracket$ é um processo contínuo e de variação limitada. Agora, pelo proposição 3.21 segue que $\mathbf{E}(\llbracket \mathbf{N} \rrbracket(\infty))<\infty$, em particular, $\llbracket \mathbf{N} \rrbracket(\infty)<\infty$, $\mathrm{P}$ - q. s. e como $\mathbf{H} \in \mathcal{L}^{2}(\mathbf{M})$, segue que $\int_{0}^{\infty} H^{2}(s, \omega) d \llbracket \mathbf{M} \rrbracket(d s, \omega)<\infty$, P - q. s. Pela desigualdade de Kunita-Watanabe* resulta que

$$
\begin{aligned}
\int_{0}^{\infty}|H(s, \omega)|\left|\mu_{\omega}^{\llbracket \mathbf{M}, \mathbf{N} \rrbracket}\right|(d s) & \leq\left(\int_{0}^{\infty} H^{2}(s, \omega) \mu_{\omega}^{\llbracket \mathbf{M} \rrbracket}(d s)\right)^{\frac{1}{2}}\left(\int_{0}^{\infty} 1^{2} \mu_{\omega}^{\llbracket \mathbf{N} \rrbracket}(d s)\right)^{\frac{1}{2}} \\
& =\left(\int_{0}^{\infty} H^{2}(s, \omega) \mu_{\omega}^{\llbracket \mathbf{M} \rrbracket}(d s)\right)^{\frac{1}{2}}(\llbracket \mathbf{N} \rrbracket(\infty))^{\frac{1}{2}} \\
& <\infty
\end{aligned}
$$

para quase todo $\omega \in \Omega$ e portanto (4.9) é válido. O fato do processo estar bem definido segue do teorema 4.1 e a continuidade e variação limitada seguem da proposição 4.4 .

O próximo resultado é fundamental. Ele garante a existência da integral estocástica de um processo em $\mathcal{L}^{2}(\mathbf{M})$ com respeito à um martingal local contínuo $\mathbf{M}$.

Teorema 4.17. Seja $\mathbf{M}$ um martingal local contínuo e $\mathbf{H} \in \mathcal{L}^{2}(\mathbf{M})$. Então existe um único martingal local contínuo $\mathbf{I}$ tal que

$$
\llbracket \mathbf{I}, \mathbf{N} \rrbracket=\mathbf{H} \bullet \llbracket \mathbf{M}, \mathbf{N} \rrbracket
$$

para qualquer martingal local contínuo $\mathbf{N}$. Denotamos $\mathbf{I} \doteq \mathbf{H} \bullet \mathbf{M}$. Além disso, $\mathbf{I} \in \mathcal{H}_{0}^{2}$, é bilinear em $\mathbf{H}$ e $\mathbf{M}$ e a aplicação $\mathbf{H} \in \mathcal{L}^{2}(\mathbf{M}) \mapsto \mathbf{H} \bullet \mathbf{M} \in c \mathcal{H}_{0}^{2}$ é uma isometria linear. É a chamada de Isometria de Itô.

\footnotetext{
* Veja o teorema 4.12 na página 74 .
} 
Demonstração. Vamos começar provando a unicidade. Suponha que existam dois martingais locais contínuos I e L ambos satisfazendo (4.10). Temos que

$$
\llbracket \mathbf{I}-\mathbf{L}, \mathbf{N} \rrbracket=\llbracket \mathbf{I}, \mathbf{N} \rrbracket-\llbracket \mathbf{L}, \mathbf{N} \rrbracket=0
$$

para qualquer martingal local contínuo N. Assim, tome em particular $\mathbf{N}=\mathbf{I}-\mathbf{L}$ em (4.11) e ficamos com $\llbracket \mathbf{I}-\mathbf{L} \rrbracket=0$. Resulta pelo teorema de Fisk* que $\mathbf{I}-\mathbf{L}$ é constante e como $I(0, \cdot)=L(0, \cdot)=0$ para todo $\omega \in \Omega$, provamos a unicidade.

Agora a existência. Vamos supor inicialmente que $\mathbf{N} \in \mathrm{cH}_{0}^{2}$. Pelas desigualdades de KunitaWatanabe e Hölder, temos que

$$
\begin{aligned}
\mid \mathbf{E}\left(\int_{0}^{\infty} H(s, \omega) \llbracket \mathbf{M}, \mathbf{N} \rrbracket(d s, \omega)\right) & \leq\left|\mathbf{E}\left(\sqrt{\int_{0}^{\infty} H^{2}(s, \omega) \llbracket \mathbf{M} \rrbracket(d s, \omega)} \sqrt{\int_{0}^{\infty} 1 \llbracket \mathbf{N} \rrbracket(d s, \omega)}\right)\right| \\
& \leq\left\|\int_{0}^{\infty} H^{2}(s, \omega) \llbracket \mathbf{M} \rrbracket(d s, \omega)\right\| \\
& =\|\mathbf{H}\|_{\mathbf{M}} \sqrt{\mathbf{E}(\llbracket \mathbf{N} \rrbracket(\infty))} .
\end{aligned}
$$

Como $\mathbf{N} \in \mathrm{cH}_{0}^{2}$, pela proposição 3.21 temos que $\mathbf{E}(\llbracket \mathbf{N} \rrbracket(\infty))<\infty$ e pelo corolário 3.22 segue que $\sqrt{\mathbf{E}(\llbracket \mathbf{N} \rrbracket(\infty))}=\|\mathbf{N}\|_{\mathcal{H}^{2}}<\infty$. Desta forma, ficamos com

$$
\left|\mathbf{E}\left(\int_{0}^{\infty} H(s, \omega) \llbracket \mathbf{M}, \mathbf{N} \rrbracket(d s, \omega)\right)\right| \leq\|\mathbf{H}\|_{\mathbf{M}}\|\mathbf{N}\|_{\mathcal{H}^{2}}<\infty,
$$

ou seja, $\int_{0}^{\infty} H(s, \omega) \llbracket \mathbf{M}, \mathbf{N} \rrbracket(d s, \omega)<\infty, \mathrm{P}-\mathrm{q}$. s. Agora considere o funcional $\Phi_{\mathbf{H}}: \mathrm{cH}_{0}^{2} \rightarrow \mathbb{R}$ definido como

$$
\Phi_{\mathbf{H}}(\mathbf{N})=\mathbf{E}\left(\int_{0}^{\infty} H(s, \omega) \llbracket \mathbf{M}, \mathbf{N} \rrbracket(d s, \omega)\right) .
$$

Por (4.12), temos que $\Phi_{\mathbf{H}}$ é limitado e portanto contínuo. Além disso, pela bilinearidade da covariação quadrática e da integral ${ }^{\dagger} \mathbf{X} \bullet \llbracket \mathbf{M}, \mathbf{N} \rrbracket$, resulta que $\Phi_{\mathbf{H}}$ é um funcional linear contínuo no espaço de Hilbert ${ }^{\ddagger} \mathrm{cH}_{0}^{2}$. Pelo teorema de representação de Riesz, existe um único elemento $\mathbf{I} \in \mathrm{cH}_{0}^{2}$

\footnotetext{
* Veja o teorema 3.6 na página 35. † Veja a proposição 4.4. $\quad$ † Veja a proposição 2.40 na página 23.
} 
tal que para qualquer $\mathbf{N} \in \mathrm{cH}_{0}^{2}$,

$$
\mathbf{E}\left(\int_{0}^{\infty} H(s, \omega) \llbracket \mathbf{M}, \mathbf{N} \rrbracket(d s, \omega)\right) \doteq \Phi_{\mathbf{H}}(\mathbf{N})=\langle\mathbf{I}, \mathbf{N}\rangle_{\mathrm{cH}^{2}}=\mathbf{E}(I(\infty) N(\infty)) .
$$

Vamos verificar agora que

$$
\llbracket \mathbf{I}, \mathbf{N} \rrbracket=\mathbf{H} \bullet \llbracket \mathbf{M}, \mathbf{N} \rrbracket .
$$

Como $\mathbf{H}$ é progressivamente mensurável, segue pelo teorema 4.1 que $\mathbf{H} \bullet \llbracket \mathbf{M}, \mathbf{N} \rrbracket$ é adaptado e pela proposição 4.4 segue que $\mathbf{H} \bullet \llbracket \mathbf{M}, \mathbf{N} \rrbracket$ é contínuo e de variação limitada. Além disso $(\mathbf{H} \bullet \llbracket \mathbf{M}, \mathbf{N} \rrbracket)(0, \omega)=$ 0 para todo $\omega \in \Omega$. Afirmamos que para provar (4.13) é suficiente provar que

$$
\mathbf{X} \doteq \mathbf{I N}-\mathbf{H} \bullet \llbracket \mathbf{M}, \mathbf{N} \rrbracket
$$

é um martingal local. De fato, I e $\mathbf{N}$ são martingais locais contínuos, segue pela proposição 3.16 que $\llbracket \mathbf{I}, \mathbf{N} \rrbracket$ é o único processo contínuo de variação limitada tal que $\mathbf{I N}-\llbracket \mathbf{I}, \mathbf{N} \rrbracket$ é um martingal local contínuo. Dessa unicidade resulta (4.13). Então nossa tarefa agora é mostrar que $\mathbf{X}$ é um martingal local contínuo. Vamos mostrar em particular que $\mathbf{X}$ é um martingal. De acordo com a proposição 2.37 na página 21 , basta mostrar que para qualquer tempo de parada limitado $\tau$, vale que $X(\tau) \in \mathrm{L}^{1}$ e $\mathbf{E}(X(\tau))=\mathbf{E}(X(0))=0$ ou, equivalentemente

$$
\mathbf{E}(I(\tau) N(\tau))=\mathbf{E}((\mathbf{H} \bullet \llbracket \mathbf{M}, \mathbf{N} \rrbracket)(\tau))
$$

De fato, temos que $(\mathbf{H} \bullet \llbracket \mathbf{M}, \mathbf{N} \rrbracket)(\tau) \in \mathrm{L}^{1}$ pois $\mathbf{H}$ satisfaz (4.9), além disso como $\mathbf{I}$ e $\mathbf{N}$ pertencem a $\mathcal{H}^{2}$, temos pela desigualdade de Doob*

$$
\mathbf{E}\left(\sup _{t \geq 0}|I(t)|^{2}\right) \leq 4 \sup _{t \geq 0} \mathbf{E}\left(I^{2}(t)\right) \doteq 4\|I\|_{\mathcal{H}^{2}}^{2}<\infty
$$

e análogamente

$$
\mathbf{E}\left(\sup _{t \geq 0}|N(t)|^{2}\right) \leq 4 \sup _{t \geq 0} \mathbf{E}\left(N^{2}(t)\right) \doteq 4\|N\|_{\mathcal{H}^{2}}^{2}<\infty
$$

e portanto $I(\tau) N(\tau) \in \mathrm{L}^{1}$. Com isso provamos que $X(\tau) \in \mathrm{L}^{1}$. Agora vamos provar a relação 4.14.

\footnotetext{
* Veja o teorema 2.27 na página 18.
} 
Para isso observe os seguintes fatos: (1) Pelo corolário 2.38, o processo parado $\mathbf{N}^{\tau}$ pertence à c $\mathcal{H}_{0}^{2} \mathrm{e}$ assim, pelo teorema de convergência de Doob* podemos considerar a variável aleatória $N^{\tau}(\infty)$ em L ${ }^{2}$ que obviamente satisfaz a relação $N^{\tau}(\infty)=N(\tau)$. (2) Como $\mathbf{I} \in \mathrm{cH}_{0}^{2}$, I é um martingal uniformemente integrável ${ }^{\dagger}$ e assim pelo teorema $2.36, I(\tau)=\mathbf{E}\left(I(\infty) \| \mathscr{F}_{\tau}\right)$. (3) Como $\mathbf{N}$ é progressivamente mensurável (pois é contínuo), temos pelo teorema 2.23 que $N(\tau)$ é $\mathscr{F}_{\tau}$-mensurável. Agora, desses fatos, temos que

$$
\mathbf{E}(I(\tau) N(\tau))=\mathbf{E}\left(\mathbf{E}\left(I(\infty) \| \mathscr{F}_{\tau}\right) N(\tau)\right)=\mathbf{E}\left(\mathbf{E}\left(I(\infty) N(\tau) \| \mathscr{F}_{\tau}\right)\right)
$$

Pelo teorema C.11 na página 161, vem

$$
\mathbf{E}\left(\mathbf{E}\left(I(\infty) N(\tau) \| \mathscr{F}_{\tau}\right)\right)=\mathbf{E}(I(\infty) N(\tau)),
$$

e assim, ficamos com

$$
\begin{aligned}
\mathbf{E}(I(\tau) N(\tau)) & =\mathbf{E}(I(\infty) N(\tau)) \\
& =\mathbf{E}\left(I(\infty) N^{\tau}(\infty)\right) \\
& \doteq \Phi_{\mathbf{H}}\left(\mathbf{N}^{\tau}\right) \\
& =\mathbf{E}\left(\left(\mathbf{H} \bullet \llbracket \mathbf{M}, \mathbf{N}^{\tau} \rrbracket\right)(\infty)\right) \\
& =\mathbf{E}\left(\left(\mathbf{H} \bullet \llbracket \mathbf{M}, \mathbf{N} \rrbracket^{\tau}\right)(\infty)\right) \\
& =\mathbf{E}\left((\mathbf{H} \bullet \llbracket \mathbf{M}, \mathbf{N} \rrbracket)^{\tau}(\infty)\right) \\
& =\mathbf{E}((\mathbf{H} \bullet \llbracket \mathbf{M}, \mathbf{N} \rrbracket)(\tau)),
\end{aligned}
$$

onde usamos as proposições 3.20 e 4.4 para justificar a quinta e sexta igualdade respectivamente.

O leitor deve estar lembrado que no início da demonstração supomos $\mathbf{N} \in \mathrm{cH}_{0}^{2}$. Para provar o teorema precisamos relaxar esta hipotése. Assim, seja $\mathbf{N}$ um martingal local contínuo tal que $N(0)=0$ para todo $\omega \in \Omega$. Pela proposição 2.52, existe uma sequência localizadora $\left\{\tau_{n}\right\}$ tal que $\mathbf{N}^{\tau_{n}}$ é uniformemente limitado, em particular, $\mathbf{N}^{\tau_{n}} \in \mathrm{cH}_{0}^{2}$ para todo $n \in \mathbb{N}$. Novamente pelas proposições 3.20 e 4.4 , temos para qualquer $n \in \mathbb{N}$

$$
\llbracket \mathbf{I}, \mathbf{N} \rrbracket^{\tau_{n}}=\llbracket \mathbf{I}, \mathbf{N}^{\tau_{\mathbf{n}}} \rrbracket=\mathbf{H} \bullet \llbracket \mathbf{M}, \mathbf{N}^{\tau_{\mathbf{n}}} \rrbracket=(\mathbf{H} \bullet \llbracket \mathbf{M}, \mathbf{N} \rrbracket)^{\tau_{n}} .
$$

\footnotetext{
* Veja o teorema 2.35 na página $20 . \quad$ † Veja o teorema B.8.
} 
Fazendo $n \rightarrow \infty$ obtemos (4.13). Finalmente, pela proposição 3.29 na página 58 - item $\mathbf{v}$ ), temos que trocando $\mathbf{N}$ por $\mathbf{N}-N(0)$ o resultado contínua válido e assim provamos o resultado para $\mathbf{N}$ martingal local qualquer.

Agora vamos provar a Isometria de Itô. Considere a aplicação $\mathbf{H} \in \mathcal{L}^{2}(\mathbf{M}) \mapsto \mathbf{I}=\mathbf{H} \bullet \mathbf{M} \in \mathrm{cH}_{0}^{2}$. Vamos inicialmente mostrar a linearidade, isto é para $\mathbf{H}_{1}, \mathbf{H}_{2} \in \mathcal{L}^{2}(\mathbf{M})$ e $\alpha, \beta \in \mathbb{R}$

$$
\left(\alpha \mathbf{H}_{1}+\beta \mathbf{H}_{2}\right) \bullet \mathbf{M}=\alpha\left(\mathbf{H}_{1} \bullet \mathbf{M}\right)+\beta\left(\mathbf{H}_{2} \bullet \mathbf{M}\right) .
$$

De fato, como $\alpha \mathbf{H}_{1}+\beta \mathbf{H}_{2} \in \mathcal{L}^{2}(\mathbf{M})$, temos que $\left(\alpha \mathbf{H}_{1}+\beta \mathbf{H}_{2}\right) \bullet \mathbf{M}$ é o único processo em c $\mathcal{H}_{0}^{2}$ tal que para qualquer martingal local contínuo $\mathbf{N}$,

$$
\begin{aligned}
\llbracket\left(\alpha \mathbf{H}_{\mathbf{1}}+\beta \mathbf{H}_{\mathbf{2}}\right) \bullet \mathbf{M}, \mathbf{N} \rrbracket & =\left(\alpha \mathbf{H}_{1}+\beta \mathbf{H}_{2}\right) \bullet \llbracket \mathbf{M}, \mathbf{N} \rrbracket \\
& =\alpha \mathbf{H}_{1} \bullet \llbracket \mathbf{M}, \mathbf{N} \rrbracket+\beta \mathbf{H}_{2} \bullet \llbracket \mathbf{M}, \mathbf{N} \rrbracket
\end{aligned}
$$

mas por outro lado,

$$
\begin{aligned}
\llbracket \alpha\left(\mathbf{H}_{1} \bullet \mathbf{M}\right)+\beta\left(\mathbf{H}_{2} \bullet \mathbf{M}\right), \mathbf{N} \rrbracket & =\llbracket \alpha\left(\mathbf{H}_{1} \bullet \mathbf{M}\right), \mathbf{N} \rrbracket+\llbracket \beta\left(\mathbf{H}_{2} \bullet \mathbf{M}\right), \mathbf{N} \rrbracket \\
& =\alpha \llbracket \mathbf{H}_{1} \bullet \mathbf{M}, \mathbf{N} \rrbracket+\beta \llbracket \mathbf{H}_{2} \bullet \mathbf{M}, \mathbf{N} \rrbracket \\
& =\alpha \mathbf{H}_{1} \bullet \llbracket \mathbf{M}, \mathbf{N} \rrbracket+\beta \mathbf{H}_{2} \bullet \llbracket \mathbf{M}, \mathbf{N} \rrbracket
\end{aligned}
$$

Donde segue a linearidade (e a bilinearidade pelo mesmo argumento). Agora, para a propriedade isométrica, como $\mathbf{I} \in \mathrm{cH}_{0}^{2}$, pelo corolário 3.22 na página 56

$$
\|\mathbf{I}\|_{\mathcal{H}^{2}}^{2}=\mathbf{E}\left(I^{2}(\infty)\right)=\Phi_{\mathbf{H}}(\mathbf{I})=\mathbf{E}((\mathbf{H} \bullet \llbracket \mathbf{M}, \mathbf{I} \rrbracket)(\infty))
$$

Como vale que $\mathbf{H} \bullet \llbracket \mathbf{M}, \mathbf{N} \rrbracket=\llbracket \mathbf{I}, \mathbf{N} \rrbracket$ para qualquer $\mathbf{N}$ martingal local contínuo, tome em particular 
$\mathbf{N}=\mathbf{I}=\mathbf{H} \bullet \mathbf{M}$. Assim e usando o teorema 4.7, temos que

$$
\begin{aligned}
\mathbf{H} \bullet \llbracket \mathbf{M}, \mathbf{I} \rrbracket & \doteq \mathbf{H} \bullet \llbracket \mathbf{M}, \mathbf{H} \bullet \mathbf{M} \rrbracket \\
& =\llbracket \mathbf{H} \bullet \mathbf{M}, \mathbf{H} \bullet \mathbf{M} \rrbracket \\
& =\mathbf{H} \bullet \llbracket \mathbf{M}, \mathbf{H} \bullet \mathbf{M} \rrbracket \\
& =\mathbf{H} \bullet \llbracket \mathbf{H} \bullet \mathbf{M}, \mathbf{M} \rrbracket \\
& =\mathbf{H} \bullet(\mathbf{H} \bullet \llbracket \mathbf{M}, \mathbf{M} \rrbracket) \\
& =\mathbf{H}^{2} \bullet \llbracket \mathbf{M} \rrbracket .
\end{aligned}
$$

Dessa maneira (4.15) fica

$$
\begin{aligned}
\|\mathbf{I}\|_{\mathcal{H}^{2}}^{2} & =\mathbf{E}((\mathbf{H} \bullet \llbracket \mathbf{M}, \mathbf{I} \rrbracket)(\infty)) \\
& =\mathbf{E}\left(\left(\mathbf{H}^{2} \bullet \llbracket \mathbf{M} \rrbracket\right)(\infty)\right) \\
& =\mathbf{E}\left(\int_{0}^{\infty} H^{2}(s, \omega) \llbracket \mathbf{M} \rrbracket(d s, \omega)\right) \\
& \doteq\|\mathbf{H}\|_{\mathbf{M}}^{2},
\end{aligned}
$$

e está provado o resultado.

Definição 4.18. Nas condições do teorema anterior, o martingal $\mathbf{H} \bullet \mathbf{M}$ é chamado de Integral de Itô de $\mathbf{H}$ com respeito a $\mathbf{M}$ e também é denotada por

$$
\int_{0} H(s) d M(s) .
$$

Observe que ainda não é claro o motivo da notação com o símbolo de uma integral. Trataremos disso adiante neste capítulo. O próximo lema será utíl na demonstração da propriedade da associatividade da integral estocástica.

Lema 4.19. Sejam $\mathbf{N}$ e $\mathbf{M}$ martingais locais contínuos e $\mathbf{H} \in \mathcal{L}^{2}(\mathbf{M})$ e $\mathbf{K} \in \mathcal{L}^{2}(\mathbf{N})$. Então

i) $\llbracket \mathbf{H} \bullet \mathbf{M}, \mathbf{K} \bullet \mathbf{N} \rrbracket(t, \omega)=\int_{0}^{t}(H K)(s, \omega) \llbracket \mathbf{M}, \mathbf{N} \rrbracket(d s, \omega)$,

ii) $\llbracket \mathbf{H} \bullet \mathbf{M} \rrbracket(t, \omega)=\int_{0}^{t}\left(H^{2}(s, \omega)\right) \llbracket \mathbf{M} \rrbracket(d s, \omega)$. 
Demonstração. Inicialmente note que ii segue de $\mathbf{i}$. Basta tomar $\mathbf{K}=\mathbf{H}$ e $\mathbf{N}=\mathbf{M}$. Vamos começar a provar i mostrando que $\mathbf{H K} \in \mathcal{L}^{1}(\llbracket \mathbf{M}, \mathbf{N} \rrbracket)$. De fato, pela desigualdade de Kunita-Watanabe,

$$
\int_{0}^{\infty}|H K(s, \omega)| \mathrm{V}_{\llbracket \mathbf{M}, \mathbf{N} \rrbracket}(d s, \omega) \leq \sqrt{\int_{0}^{\infty} H^{2}(s, \omega) \llbracket \mathbf{M} \rrbracket(d s, \omega)} \sqrt{\int_{0}^{\infty} K^{2}(s, \omega) \llbracket \mathbf{N} \rrbracket(d s, \omega)}<\infty,
$$

para quase todo $\omega \in \Omega$.

Agora seja $\mu_{\omega}^{\llbracket \mathbf{H} \bullet \mathbf{M}, \mathbf{N} \rrbracket}$ a medida gerada por $\llbracket \mathbf{H} \bullet \mathbf{M}, \mathbf{N} \rrbracket$. Temos que

$$
\begin{aligned}
\mu_{\omega}^{\llbracket \mathbf{H} \bullet \mathbf{M}, \mathbf{N} \rrbracket}([0, t]) & =\llbracket \mathbf{H} \bullet \mathbf{M}, \mathbf{N} \rrbracket(t)-\llbracket \mathbf{H} \bullet \mathbf{M}, \mathbf{N} \rrbracket(0) \\
& =\mathbf{H} \bullet \llbracket \mathbf{M}, \mathbf{N} \rrbracket(t) \\
& =\int_{0}^{t} H(s, \omega) \llbracket \mathbf{M}, \mathbf{N} \rrbracket(d s, \omega) \\
& \doteq \int_{0}^{t} H(s, \omega) \mu_{\omega}^{\llbracket \mathbf{M}, \mathbf{N} \rrbracket}(d s) .
\end{aligned}
$$

Temos que $H(s, \cdot)=\frac{d \mu_{\omega}^{\llbracket \mathbf{H} \bullet \mathbf{M}, \mathbf{N} \rrbracket}}{d \mu_{\omega}^{\llbracket M, N \rrbracket}}$ e portanto resulta

$$
\begin{aligned}
\int_{0}^{t}(H K)(s, \omega) \mu_{\omega}^{\llbracket \mathbf{M}, \mathbf{N} \rrbracket}(d s) & =\int_{0}^{t} K(s, \omega) \mu_{\omega}^{\llbracket \mathbf{H} \bullet \mathbf{M}, \mathbf{N} \rrbracket} \\
& =\mathbf{K} \bullet \llbracket \mathbf{H} \bullet \mathbf{M}, \mathbf{N} \rrbracket \\
& =\mathbf{K} \bullet \llbracket \mathbf{N}, \mathbf{H} \bullet \mathbf{M} \rrbracket \\
& =\llbracket \mathbf{K} \bullet \mathbf{N}, \mathbf{H} \bullet \mathbf{M} \rrbracket .
\end{aligned}
$$

Teorema 4.20. Seja $\mathbf{M}$ um martingal local contínuo, $\mathbf{K} \in \mathcal{L}^{2}(\mathbf{M})$ e $\mathbf{H} \in \mathcal{L}^{2}(\mathbf{K} \bullet \mathbf{M})$. Então $\mathbf{H K} \in \mathcal{L}^{2}(\mathbf{M})$ e vale a propriedade associativa: $\mathbf{H} \bullet(\mathbf{K} \bullet \mathbf{M})=(\mathbf{H K}) \bullet \mathbf{M}$.

Demonstração. Seja $\mu_{\omega}^{\llbracket \mathbf{K} \bullet \mathbf{M} \rrbracket}$ a medida gerada pelas trajetórias do processo $\llbracket \mathbf{K} \bullet \mathbf{M} \rrbracket$. Temos pelo 
lema anterior que

$$
\begin{aligned}
& \mu_{\omega}^{\llbracket \mathbf{K} \bullet \mathbf{M} \rrbracket}([0, t])=\llbracket \mathbf{K} \bullet \mathbf{M} \rrbracket(t) \\
& =\int_{0}^{t} K^{2}(s, \omega) \llbracket \mathbf{M} \rrbracket(d s, \omega) \\
& \doteq \int_{0}^{t} K^{2}(s, \omega) \mu_{\omega}^{\llbracket \mathbf{M} \rrbracket}(d s) .
\end{aligned}
$$

Assim, $K^{2}(s, \cdot)=\frac{d \mu_{\omega}^{\llbracket K \bullet M} \rrbracket}{d \mu_{\omega}^{\llbracket M} \rrbracket}$. Temos portanto que

$$
\begin{aligned}
\|\mathbf{H K}\|_{\mathbf{M}}^{2} & =\mathbf{E}\left(\int_{0}^{\infty}((H K)(s, \omega))^{2}\right) \mu_{\omega}^{\llbracket \mathbf{M} \rrbracket}(d s) \\
& =\mathbf{E}\left(\int_{0}^{\infty}(H(s, \omega))^{2}\right) \mu_{\omega}^{\llbracket \mathbf{K} \bullet \mathbf{M} \rrbracket}(d s) \\
& =\|\mathbf{H}\|_{\mathbf{K} \bullet \mathbf{M}}^{2}<\infty .
\end{aligned}
$$

Segue que $\mathbf{H K} \in \mathcal{L}^{2}(\mathbf{M})$ e pelo teorema 4.17 , existe um único processo $\mathbf{H K} \bullet \mathbf{M} \in \mathrm{cH}_{0}^{2}$, tal que para qualquer martingal local contínuo $\mathbf{N}$

$$
\llbracket \mathbf{H K} \bullet \mathrm{M}, \mathrm{N} \rrbracket=\mathbf{H K} \bullet \llbracket \mathrm{M}, \mathbf{N} \rrbracket .
$$

Agora, note que como $\mathbf{K} \in \mathcal{L}^{2}(\mathbf{M})$ e $\mathbf{H} \in \mathcal{L}^{2}(\mathbf{K} \bullet \mathbf{M})$, segue pelo lema 4.16 que $\mathbf{K} \in \mathcal{L}_{\text {loc }}^{1}(\llbracket \mathbf{M}, \mathbf{N} \rrbracket)$ e $\mathbf{H} \in \mathcal{L}_{\text {loc }}^{1}(\llbracket \mathbf{K} \bullet \mathbf{M}, \mathbf{N} \rrbracket)$ ou seja $\mathbf{H} \in \mathcal{L}_{\text {loc }}^{1}(\mathbf{K} \bullet \llbracket \mathbf{M}, \mathbf{N} \rrbracket)$. Estamos sob as hipóteses do teorema 4.7 e, portanto

$$
\llbracket \mathbf{H K}, \bullet \mathbf{N} \rrbracket=\mathbf{H K} \bullet \llbracket \mathbf{M}, \mathbf{N} \rrbracket=\mathbf{H} \bullet(\mathbf{K} \bullet \llbracket \mathbf{M}, \mathbf{N} \rrbracket)=\mathbf{H} \bullet \llbracket \mathbf{K} \bullet \mathbf{M}, \mathbf{N} \rrbracket \text {. }
$$

Mas, $\mathbf{H} \bullet \mathbf{K} \bullet \mathbf{M}$ é o único processo tal que $\llbracket \mathbf{H} \bullet(\mathbf{K} \bullet \mathbf{M}), \mathbf{N} \rrbracket=\mathbf{H} \bullet \llbracket \mathbf{K} \bullet \mathbf{M}, \mathbf{N} \rrbracket$. Disso resulta $\mathbf{H K} \bullet \mathbf{M}=\mathbf{H} \bullet \mathbf{K} \bullet \mathbf{M}$, como queríamos.

Nosso objetivo é estender a integral de Itô para integradores que sejam semi-martingais. O primeiro passo será estender a classe dos processos integrantes. Para isso, precisaremos da seguinte 
propriedade:

Proposição 4.21. Sejam $\mathbf{M}$ um martingal local contínuo, $\mathbf{H} \in \mathcal{L}^{2}(\mathbf{M})$ e $\tau$ um tempo de parada arbitrário, então*

$$
\mathbf{H}^{\tau} \bullet \mathbf{M}^{\tau}=\mathbf{H} \bullet\left(\mathbf{M}^{\tau}\right)=\left(\mathbb{1}_{[0, \tau]} \mathbf{H}\right) \bullet \mathbf{M}=(\mathbf{H} \bullet \mathbf{M})^{\tau}
$$

Demonstração. Seja $\mathbf{N}$ um martingal local contínuo arbitrário.

i) $\mathbf{H} \bullet \mathbf{M}^{\tau}=(\mathbf{H} \bullet \mathbf{M})^{\tau}$.

Pela proposição 3.20 , temos que

$$
\llbracket(\mathbf{H} \bullet \mathbf{M})^{\tau}, \mathbf{N} \rrbracket=\llbracket \mathbf{H} \bullet \mathbf{M}, \mathbf{N}^{\tau} \rrbracket=\mathbf{H} \bullet \llbracket \mathbf{M}, \mathbf{N}^{\tau} \rrbracket=\mathbf{H} \bullet \llbracket \mathbf{M}^{\tau}, \mathbf{N} \rrbracket
$$

Por outro lado, como $\mathbf{H} \bullet \mathbf{M}^{\tau}$ é o único processo tal que

$$
\llbracket \mathbf{H} \bullet \mathbf{M}^{\tau}, \mathbf{N} \rrbracket=\mathbf{H} \bullet \llbracket \mathbf{M}^{\tau}, \mathbf{N} \rrbracket,
$$

segue que $\mathbf{H} \bullet \mathbf{M}^{\tau}=(\mathbf{H} \bullet \mathbf{M})^{\tau}$.

ii) $\mathbf{H}^{\tau} \bullet \mathbf{M}^{\tau}=(\mathbf{H} \bullet \mathbf{M})^{\tau}$.

Basta observar que, novamente, pela proposição 3.20, vem

$$
\llbracket \mathbf{H}^{\tau} \bullet \mathbf{M}^{\tau}, \mathbf{N} \rrbracket=\mathbf{H}^{\tau} \bullet \llbracket \mathbf{M}^{\tau}, \mathbf{N} \rrbracket=\mathbf{H}^{\tau} \bullet \llbracket \mathbf{M}, \mathbf{N} \rrbracket^{\tau}
$$

e como $\tau$ é arbitrário, da unicidade da integral estocástica de Itô resulta $\mathbf{H}^{\tau} \bullet \mathbf{M}^{\tau}=(\mathbf{H} \bullet \mathbf{M})^{\tau}$.

iii) $\left(\mathbb{1}_{[0, \tau]} \mathbf{H}\right) \bullet \mathbf{M}=(\mathbf{H} \bullet \mathbf{M})^{\tau}$.

Observe que $\mathbb{1}_{[0, \tau]} \mathbf{H} \in \mathcal{L}^{2}(\mathbf{M})$ e que $\llbracket \mathbf{M}, \mathbf{N} \rrbracket$ é um processo de variação limitada. Podemos então usar a proposição 4.4-iii e concluir que

$$
\begin{aligned}
\llbracket \mathbf{H} \bullet \mathbf{M}^{\tau}, \mathbf{N} \rrbracket & =\mathbf{H} \bullet \llbracket \mathbf{M}^{\tau}, \mathbf{N} \rrbracket \\
& =\mathbf{H} \bullet \llbracket \mathbf{M}, \mathbf{N} \rrbracket^{\tau} \\
& =\mathbb{1}_{[0, \tau]} \mathbf{H} \bullet \llbracket \mathbf{M}, \mathbf{N} \rrbracket \\
& =\llbracket \mathbb{1}_{[\mathbf{0}, \tau]} \mathbf{H} \bullet \mathbf{M}, \mathbf{N} \rrbracket .
\end{aligned}
$$

\footnotetext{
* Lembre a definição de intervalo estocástico [0, $\tau]$. Definição 4.3 na página 65.
} 
Agora, da bilineridade da co-variação quadrática, resulta que para qualquer martingal local contínuo $\mathbf{N}$

$$
\llbracket \mathbf{H} \bullet \mathbf{M}^{\tau}-\mathbb{1}_{[\mathbf{0}, \tau]} \mathbf{H} \bullet \mathbf{M}, \mathbf{N} \rrbracket=0,
$$

em particular

$$
\llbracket \mathbf{H} \bullet \mathbf{M}^{\tau}-\mathbb{1}_{[\mathbf{0}, \tau]} \mathbf{H} \bullet \mathbf{M} \rrbracket=0 .
$$

Assim, pelo teorema de Fisk* e do fato dos processos se anularem em $t=0$, resulta a igualdade desejada.

\section{A integral de Itô para martingais locais contínuos estendida}

Seja M um martingal local contínuo. Vamos definir um espaço maior para nossos integrandos.

Definição 4.22. Definimos o espaço $\mathcal{L}_{\text {loc }}^{2}(\mathbf{M})$ como o conjunto de todos processos progressivamente mensuráveis $\mathbf{H}$ tais que existe uma sequência localizadora $\left\{\tau_{n}\right\}_{n \geq 1}$ de tempos de parada tal que

$$
\mathbf{E}\left(\int_{0}^{\tau_{n}} H^{2}(s, \omega) \llbracket \mathbf{M} \rrbracket(d s, \omega)\right)<\infty .
$$

Note que pelo Teorema 4.21, temos

$$
\begin{aligned}
\mathbf{E}\left(\int_{0}^{\tau_{n}} H^{2}(s, \omega) \llbracket \mathbf{M} \rrbracket(d s, \omega)\right) & =\mathbf{E}\left(\int_{0}^{\infty} \mathbb{1}_{\left[0, \tau_{n}\right]} H^{2}(s, \omega) \llbracket \mathbf{M} \rrbracket(d s, \omega)\right) \\
& =\mathbf{E}\left(\mathbb{1}_{\left[0, \tau_{n}\right]} \mathbf{X}^{2} \bullet \mathbf{M}\right) \\
& =\mathbf{E}\left(\mathbf{X}^{2} \bullet \mathbf{M}^{\tau_{n}}\right) \\
& =\mathbf{E}\left(\int_{0}^{\infty} X^{2}(s, \omega) \llbracket \mathbf{M} \rrbracket^{\tau_{n}}\right) \\
& =\mathbf{E}\left(\int_{0}^{\infty} X^{2}(s, \omega) \llbracket \mathbf{M}^{\tau_{\mathbf{n}}} \rrbracket\right),
\end{aligned}
$$

\footnotetext{
* Veja o teorema 3.6 na página 35.
} 
onde na última igualdade usamos a proposição 3.13 na página 47. Essas igualdades explicitam algumas condições equivalentes para um processo pertencer à classe $\mathcal{L}_{\text {loc }}^{2}(\mathbf{M})$. Observamos também a inclusão $\mathcal{L}^{2}(\mathbf{M}) \subseteq \mathcal{L}_{\text {loc }}^{2}(\mathbf{M})$. Vamos dar mais uma caracterização de processos em $\mathcal{L}_{\text {loc }}^{2}(\mathbf{M})$.

Proposição 4.23. Seja $\mathbf{H}$ um processo progressivamente mensurável e $\mathbf{M}$ um martingal local contínuo. Se $\int_{0}^{t} H^{2}(s, \omega) \llbracket \mathbf{M} \rrbracket(d s, \omega)<\infty$, P-q. s. para cada $t \geq 0$ então $\mathbf{H} \in \mathcal{L}_{\text {loc }}^{2}(\mathbf{M})$.

Demonstração. Pela proposição 4.4, temos que o processo $\mathbf{H}^{2} \bullet \llbracket \mathbf{M} \rrbracket$ é contínuo e se anula em $t=0$. Defina os tempos de parada

$$
\tau_{n} \doteq \inf _{t \geq 0}\left\{\int_{0}^{t} H^{2}(s, \omega) \llbracket \mathbf{M} \rrbracket(d s, \omega) \text { ou }\left|M_{t}\right|>n\right\}
$$

Temos que $\tau_{n} \uparrow \infty$ e $\int_{0}^{\tau_{n}} H^{2}(s, \omega) \llbracket \mathbf{M} \rrbracket(d s, \omega)$, P-q.s. Para $n>\left|M_{0}\right|, \mathbf{M}^{\tau_{n}}$ é um martingal local contínuo com $\left|\mathbf{M}^{\tau_{n}}\right| \leq n$. Pelo corolário 2.56 na página 31 temos que $M^{\tau_{n}}$ é um martingal, alem disso, da limitação uniforme também resulta que $\mathbf{M}^{\tau_{n}} \in \mathrm{cH}^{2}$. Assim, $\|\mathbf{H}\|_{\mathbf{M}^{\tau_{n}}}^{2}=\mathbf{E}\left(\int_{0}^{\tau_{n}} H^{2}(s, \omega) \llbracket \mathbf{M} \rrbracket(s, \omega)\right) \leq$ $n$ e consequentemente temos para todo $n \geq 1$ que $\mathbf{H} \in \mathcal{L}^{2}\left(\mathbf{M}^{\tau_{n}}\right)$. Disso resulta que $\mathbf{H} \in \mathcal{L}_{\text {loc }}^{2}(\mathbf{M})$.

O objetivo agora será definir a integral estocástica para processos $\mathbf{H} \in \mathcal{L}_{\text {loc }}^{2}(\mathbf{M})$. Antes precisamos de um lema.

Lema 4.24. Seja $\left\{\mathbf{X}_{n}\right\}$ uma sequência de martingais contínuos e $\left\{\tau_{n}\right\}$ uma sequência de tempos de parada tais que $\tau_{n} \uparrow \infty \mathrm{P}$-q. s. e $\mathbf{X}_{n+1}^{\tau_{n}}=\mathbf{X}_{n}, n \geq 1$. Então existe um único processo adaptado $\mathbf{X}$ tal que para todo $n \geq 1, \mathbf{X}^{\tau_{n}}=\mathbf{X}_{n}$. Além disso, $\mathbf{X}$ é um martingal local contínuo.

Demonstração. Vamos provar primeiro a existência. Seja $\Lambda$ um conjunto de medida nula tal que para $\omega \in \Lambda^{c}$, temos que $\tau_{n}(\omega) \uparrow \infty$ e $X_{n+1}^{\tau_{n}}(t, \omega)=X_{n}(t, \omega)$, para todo $t \geq 0, n \geq 1$. Considere

$$
\left[0, \tau_{n}\right] \cap\left([0, \infty) \times \Lambda^{c}\right) \doteq\left\{(t, \omega) \in[0, \infty) \times \Omega: 0 \leq t \leq \tau_{n}(\omega)\right\} \cap\left\{(t, \omega) \in[0, \infty) \times \Lambda^{c}\right\}
$$

Temos que $\left[0, \tau_{n}\right] \cap\left([0, \infty) \times \Lambda^{c}\right) \uparrow\left([0, \infty) \times \Lambda^{c}\right)$ quando $n$ cresce. Assim, defina $\mathbf{X}:([0, \infty) \times \Omega) \rightarrow \mathbb{R}$ por

$$
\mathbf{X} \doteq \begin{cases}\mathbf{X}_{n} & \text { em }\left[0, \tau_{n}\right] \cap\left([0, \infty) \times \Lambda^{c}\right) \\ 0 & \text { em }([0, \infty) \times \Omega) \backslash\left([0, \infty) \times \Lambda^{c}\right)\end{cases}
$$


Dessa forma, temos que $X(t)=X_{n}(t)$ para $\omega \in\left[t \geq \tau_{n}\right] \cap\left([0, \infty) \times \Lambda^{c}\right)$, e assim

$$
X(t, \omega)=\lim _{n \rightarrow \infty} X_{n}(t, \omega)
$$

para $\omega \in \Lambda^{c}$, ou seja, P-q. s. Como cada $X_{n}(t)$ é $\mathscr{F}_{t}$-mensurável e $\mathscr{F}_{t}$ contém os conjuntos de medida nula, resulta que $X(t)$ também é $\mathscr{F}_{t}$-mensurável. Ou seja, provamos que $\mathbf{X}$ assim definido é adaptado. Pela sua própria construção, para $\omega \in \Lambda^{c}$ as trajétorias de $\mathbf{X}$ são as trajétorias de $\mathbf{X}_{n}$ nos intervalos $\left[0, \tau_{n}\right]$ e portanto contínuas, além disso, como $\mathbf{X}$ é constante caso $\omega \notin \Lambda^{c}$, resulta que o processo $\mathbf{X}$ é contínuo. Agora note que a condição $\mathbf{X}_{n+1}^{\tau_{n}}=\mathbf{X}_{n}$ implica que $\mathbf{X}_{n}^{\tau_{n}}=\mathbf{X}_{n}$, disso e da construção de $\mathbf{X}$ resulta que para cada $t \geq 0$ e $\omega \in \Lambda^{c}, X^{\tau_{n}}(t)=X_{n}^{\tau_{n}}(t)=X_{n}(t)$. Resulta como queríamos que $\mathbf{X}^{\tau_{n}}=\mathbf{X}_{n}$. Como por hipótese $\left\{\mathbf{X}_{n}\right\}$ é um martingal temos que $\mathbf{X}$ é de fato um martingal local.

Para provar a unicidade, suponha que $\mathbf{X}$ e $\mathbf{Y}$ sejam dois processos satisfazendo $\mathbf{X}^{\tau_{n}}=\mathbf{X}_{n}=\mathbf{Y}^{\tau_{n}}$, $n \geq 1$. Pelo resultado de existência, isso implica que para qualquer $t \geq 0, X^{\tau_{n}}(t)=X_{n}^{\tau_{n}}=Y^{\tau_{n}}(t)$ em $\Lambda^{c}$, isto é, P-q. s. Fazendo $n \uparrow \infty$, resulta que $X(t)=Y(t)$, P-q. s.. Isso implica que os processos são indistinguíveis*.

Teorema 4.25. Sejam $\mathbf{M}$ um martingal local contínuo e $\mathbf{H} \in \mathcal{L}_{\text {loc }}^{2}(\mathbf{M})$. Então $\mathbf{H} \in \mathcal{L}_{\text {loc }}^{1}(\llbracket \mathbf{M}, \mathbf{N} \rrbracket)$ para cada martingal local contínuo $\mathbf{N}$ e existe um único martingal local contínuo denotado por $\mathbf{H} \bullet \mathbf{M}$ tal que $(\mathbf{H} \bullet \mathbf{M})(0)=0$ para todo $\omega \in \Omega e \llbracket \mathbf{H} \bullet \mathbf{M}, \mathbf{N} \rrbracket=\mathbf{H} \bullet \llbracket \mathbf{M}, \mathbf{N} \rrbracket$ para qualquer martingal local contínuo $\mathbf{N}$.

Demonstração. Em primeiro lugar, temos pela desigualdade de Kunita-Watanabe e pelas observações feitas após a definição do espaço $\mathcal{L}_{\text {loc }}^{2}(\mathbf{M})$, que para qualquer $t \geq 0$ e quase todo $\omega \in \Omega$,

$$
\int_{0}^{t}|H(s, \omega)| \mathrm{V}_{\llbracket \mathbf{M}, \mathbf{N} \rrbracket}(d s, \omega) \leq(\llbracket \mathbf{N} \rrbracket(t))^{\frac{1}{2}}\left(\int_{0}^{t} H^{2}(s, \omega) \llbracket \mathbf{M} \rrbracket(d s, \omega)\right)^{\frac{1}{2}}<\infty
$$

e portanto $\mathbf{H} \in \mathcal{L}_{\text {loc }}^{1}(\llbracket \mathbf{M}, \mathbf{N} \rrbracket)$. A unicidade do processo $\mathbf{H} \bullet \mathbf{M}$ é provada da mesma forma que no teorema 4.17 .

Para a existência, note que como $\mathbf{H} \in \mathcal{L}_{\text {loc }}^{2}(\mathbf{M})$, existe uma sequência localizadora de tempos de parada $\left\{\tau_{n}\right\}$ tal que $\mathbf{H} \in \mathcal{L}^{2}\left(\mathbf{M}^{\tau_{n}}\right)$, para todo $n \geq 1$. Assim pelo teorema 4.17 a sequência de processos $\left\{\mathbf{I}_{n}\right\}$, onde $\mathbf{I}_{n} \doteq \mathbf{H} \bullet \mathbf{M}^{\tau_{n}}$ é tal que $\mathbf{I}_{n} \in \mathrm{cH}_{0}^{2}$, para cada $n \geq 1$. Além disso, pela

\footnotetext{
* Veja a proposição 2.3 .
} 
proposição 4.21 temos para todo $n \geq 1$,

$$
\begin{aligned}
\mathbf{I}_{n+1}^{\tau_{n}} & =\left(\mathbf{H} \bullet \mathbf{M}^{\tau_{n+1}}\right)^{\tau_{n}} \\
& =\mathbf{H} \bullet\left(\mathbf{M}^{\tau_{n+1}}\right)^{\tau_{n}} \\
& =\mathbf{H} \bullet \mathbf{M}^{\tau_{n}} \\
& \doteq \mathbf{I}_{n} .
\end{aligned}
$$

Assim, pelo lema anterior, existe um martingal local contínuo $\mathbf{I}$ tal que $\mathbf{I}^{\tau_{n}}=\mathbf{I}_{n}=\mathbf{H} \bullet \mathbf{M}^{\tau_{n}}$. Logo, para qualquer martingal local contínuo $\mathbf{N}$, temos pelas proposições 3.20 e 4.21 que

$$
\begin{aligned}
\llbracket \mathbf{I}, \mathbf{N} \rrbracket^{\tau_{n}} & =\llbracket \mathbf{I}^{\tau_{\mathbf{n}}}, \mathbf{N} \rrbracket=\llbracket \mathbf{H} \bullet \mathbf{M}^{\tau_{\mathbf{n}}}, \mathbf{N} \rrbracket \\
& =\mathbf{H} \bullet \llbracket \mathbf{M}^{\tau_{\mathbf{n}}}, \mathbf{N} \rrbracket=\mathbf{H} \bullet \llbracket \mathbf{M}, \mathbf{N} \rrbracket^{\tau_{n}} \\
& =(\mathbf{H} \bullet \llbracket \mathbf{M}, \mathbf{N} \rrbracket)^{\tau_{n}} .
\end{aligned}
$$

Como esta igualdade vale para qualquer $n \geq 1$, fazendo $n \uparrow \infty$, resulta que

$$
\llbracket \mathbf{I}, \mathbf{N} \rrbracket=\llbracket \mathbf{H} \bullet \mathbf{M}, \mathbf{N} \rrbracket=\mathbf{H} \bullet \llbracket \mathbf{M}, \mathbf{N} \rrbracket
$$

Observe que se $\mathbf{M} \in \mathrm{cH}^{2}$ então $\mathcal{L}^{2}(\mathbf{M}) \subseteq \mathcal{L}_{\text {loc }}^{2}(\mathbf{M})$ e para cada $\mathbf{H} \in \mathcal{L}^{2}(\mathbf{M})$ as integrais definidas nos teoremas 4.17 e 4.25 coincidem. Por este motivo, obtemos

Teorema 4.26. Sejam $\mathbf{M}, \mathbf{N}$ martingais locais contínuos, $\mathbf{H} \in \mathcal{L}_{\text {loc }}^{2}$ e $\tau$ um tempo de parada. Então $\mathbf{H} \bullet \mathbf{M}$ é bilinear em $\mathbf{H}$ e $\mathbf{M} e$

$$
\mathbf{H}^{\tau} \bullet \mathbf{M}^{\tau}=\mathbf{H} \bullet\left(\mathbf{M}^{\tau}\right)=\left(\mathbb{1}_{[0, \tau]} \mathbf{H}\right) \bullet \mathbf{M}=(\mathbf{H} \bullet \mathbf{M})^{\tau}
$$

Agora vamos provar que também vale a propriedade associativa. Procedemos como na subseção anterior. A demonstração do próximo lema é igual a demonstracão do lema 4.19 e por isso será omitida.

Lema 4.27. Sejam $\mathbf{N}$ e $\mathbf{M}$ martingais locais contínuos e $\mathbf{H} \in \mathcal{L}_{\text {loc }}^{2}(\mathbf{M})$ e $\mathbf{K} \in \mathcal{L}_{\text {loc }}^{2}(\mathbf{N})$. Então

i) $\llbracket \mathbf{H} \bullet \mathbf{M}, \mathbf{K} \bullet \mathbf{N} \rrbracket(t, \omega)=\int_{0}^{t}(H K)(s, \omega) \llbracket \mathbf{M}, \mathbf{N} \rrbracket(d s, \omega)$, 
ii) $\llbracket \mathbf{H} \bullet \mathbf{M} \rrbracket(t, \omega)=\int_{0}^{t}\left(H^{2}(s, \omega)\right) \llbracket \mathbf{M} \rrbracket(d s, \omega)$.

Teorema 4.28. Seja $\mathbf{M}$ um martingal local contínuo, $\mathbf{K} \in \mathcal{L}_{\text {loc }}^{2}(\mathbf{M})$ e $\mathbf{H} \in \mathcal{L}_{l o c}^{2}(\mathbf{K} \bullet \mathbf{M})$. Então $\mathbf{H K} \in \mathcal{L}_{\text {loc }}^{2}(\mathbf{M})$ e vale a propriedade associativa: $\mathbf{H} \bullet(\mathbf{K} \bullet \mathbf{M})=(\mathbf{H K}) \bullet \mathbf{M}$.

Demonstração. Da mesma maneira como na demonstração do teorema 4.20 , seja $K^{2}(s, \cdot)=\frac{d \mu_{\omega}^{\llbracket \mathbb{M} \rrbracket}}{d \mu_{\omega}^{\llbracket \mathbb{M} \rrbracket}}$. Assim,

$$
\int_{0}^{t}(H K)^{2}(s, \omega) \mu_{\omega}^{\llbracket \mathbf{M} \rrbracket}(d s)=\int_{0}^{t} H^{2}(s, \omega) \mu_{\omega}^{\llbracket \mathbf{K} \bullet \mathbf{M} \rrbracket}(d s),
$$

como $\mathbf{H} \in \mathcal{L}_{\text {loc }}^{2}(\mathbf{K} \bullet \mathbf{M})$ segue que a última integral é finita q.s. para qualquer $t \geq 0$, e portanto $\mathbf{H K} \in \mathcal{L}_{\text {loc }}^{2}(\mathbf{M})$. Assim, pelo teorema 4.25 , o processo $(\mathbf{H K}) \bullet \mathbf{M}$ está bem definido e é um martingal local contínuo. Ainda por este mesmo teorema, segue que $\mathbf{K} \in \mathcal{L}_{\text {loc }}^{1}(\llbracket \mathbf{M}, \mathbf{N} \rrbracket)$ e $\mathbf{H} \in \mathcal{L}_{\text {loc }}^{1}(\llbracket \mathbf{K} \bullet \mathbf{M}, \mathbf{N} \rrbracket)$. Agora, o resultado segue como no teorema 4.20.

Vamos introduzir um outro espaço, importante para aplicações no capítulo 5.

Definição 4.29. Seja $\mathbf{M}$ um martingal local contínuo. Definimos o espaço $\Lambda^{2}(\mathbf{M})$ como o espaço de todos os processos progressivamente mensuráveis $\mathbf{H}$ tais que

$$
\mathbf{E}\left(\int_{0}^{t}\left(H^{2}(s, \omega)\right) \llbracket \mathbf{M} \rrbracket(d s, \omega)\right)<\infty, \quad \forall t \geq 0
$$

ou, equivalentemente

$$
\mathbf{E}\left(\int_{0}^{n}\left(H^{2}(s, \omega)\right) \llbracket \mathbf{M} \rrbracket(d s, \omega)\right)=\left\|\mathbb{1}_{[0, n]} \mathbf{H}\right\|_{\mathbf{M}}^{2}<\infty, \quad \forall n \geq 1 .
$$

Note que $\Lambda^{2}(\mathbf{M})$ é o espaço de todos os processos progressivamente mensuráveis tais que $\mathbb{1}_{[0, n]} \mathbf{H} \in$ $\mathcal{L}^{2}(\mathbf{M})$. Se $\mathbf{H} \in \Lambda^{2}(\mathbf{M})$ então* $^{*} \mathbb{1}_{[0, n]} \mathbf{H} \in \mathcal{L}^{2}(\mathbf{M})$ e portanto $(\mathbf{H} \bullet \mathbf{M})^{n}=\left(\mathbb{1}_{[0, n]} \mathbf{H}\right) \bullet \mathbf{M} \in \mathcal{H}_{0}^{2}$, para todo $n \geq 1$. Disso segue que $\mathbf{H} \bullet \mathbf{M}$ é um martingal de quadrado integrável.

\footnotetext{
* Veja a proposição 4.21 .
} 


\section{A integral de Itô para semi-martingais contínuos}

Seja $\mathbf{X}$ um semi-martingal contínuo. Lembramos que isto significa que $\mathbf{X}$ admite a decomposição

$$
\mathbf{X}=\mathbf{M}+\mathbf{A}
$$

onde $\mathbf{M}$ é um martingal local contínuo, $\mathbf{A}$ é um processo adaptado contínuo à direita de variação finita que se anula em $t=0$ e esta decomposição é única*.

Definição 4.30. Definimos o espaço $\mathcal{L}(\mathbf{X})$ como o conjunto de todos os processos progressivamente mensuráveis $\mathbf{H}$ tais que

$$
\int_{0}^{t} H^{2}(s, \omega) \llbracket \mathbf{M} \rrbracket(d s, \omega)+\int_{0}^{t}|H(s, \omega)| \mathrm{V}_{\mathbf{A}}(d s, \omega)<\infty
$$

para quase todo $\omega \in \Omega$ e todo $t \geq 0$. Em outras palavras, $\mathcal{L}(\mathbf{X}) \doteq \mathcal{L}_{\text {loc }}^{2}(\mathbf{M}) \cap \mathcal{L}_{\text {loc }}^{1}(\mathbf{A})$. Para $\mathbf{H} \in \mathcal{L}(\mathbf{X})$, definimos a integral estocástica de Itô $\mathbf{H} \bullet \mathbf{X}$ por

$$
\mathbf{H} \bullet \mathbf{X}=\mathbf{H} \bullet \mathbf{M}+\mathbf{H} \bullet \mathbf{A} .
$$

Assim, para o caso de um integrador $\mathbf{X}$ semi-martingal, o tratamento da integral estocástica é reduzido aos casos previamente estudados. Note que como $\mathbf{H} \bullet \mathbf{M}$ é martingal local e $\mathbf{H} \bullet \mathbf{A}$ é processo adaptado contínuo à direita de variação limitada segue que $\mathbf{H} \bullet \mathbf{X}$ é um semi-martingal contínuo. Vamos reunir as principais propriedades da integral estocástica para semi-martingais contínuos.

Teorema 4.31. Sejam $\mathbf{X}, \mathbf{Y}$ semi-martingais contínuos, $\mathbf{H} \in \mathcal{L}(\mathbf{X})$ e $\tau$ um tempo de parada. Então $\mathbf{H} \bullet \mathbf{M}$ é um semi-martingal contínuo, nulo em $t=0$, bilinear em $\mathbf{H}$ e $\mathbf{M}$, e valem

$$
\begin{aligned}
& \mathbf{H}^{\tau} \bullet \mathbf{M}^{\tau}=\mathbf{H} \bullet\left(\mathbf{M}^{\tau}\right)=\left(\mathbb{1}_{[0, \tau]} \mathbf{H}\right) \bullet \mathbf{M}=(\mathbf{H} \bullet \mathbf{M})^{\tau}, \\
& \llbracket \mathbf{H} \bullet \mathbf{X}, \mathbf{K} \bullet \mathbf{Y} \rrbracket(t, \omega)=\int_{0}^{t}(H K)(s, \omega) \llbracket \mathbf{X}, \mathbf{Y} \rrbracket(d s, \omega),
\end{aligned}
$$

\footnotetext{
* Veja a proposição 3.24 na página 56.
} 


$$
\llbracket \mathbf{H} \bullet \mathbf{X} \rrbracket(t, \omega)=\int_{0}^{t}\left(H^{2}(s, \omega)\right) \llbracket \mathbf{X} \rrbracket(d s, \omega)
$$

e também é válida a propriedade $\llbracket \mathbf{H} \bullet \mathbf{X}, \mathbf{Y} \rrbracket=\mathbf{H} \bullet \llbracket \mathbf{X}, \mathbf{Y} \rrbracket$.

Demonstração. Essas propriedades seguem, basicamente, combinando o teorema 4.26, proposição 4.4 e o lema 4.27. Vamos explicitar a demonstração da última propriedade citada. De fato, considere as decomposições $\mathbf{X}=\mathbf{M}+\mathbf{A}$ e $\mathbf{Y}=\mathbf{N}+\mathbf{B}$. Temos que $\mathbf{H} \in \mathcal{L}_{\text {loc }}^{2}(\mathbf{M}) \cap \mathcal{L}_{\text {loc }}^{1}(\mathbf{A})$. Disso vem que

$$
\llbracket \mathbf{H} \bullet \mathbf{X}, \mathbf{Y} \rrbracket=\llbracket \mathbf{H} \bullet \mathbf{M}+\mathbf{H} \bullet \mathbf{A}, \mathbf{N}+\mathbf{B} \rrbracket .
$$

Agora, pela proposição 3.29 na página 58 - item v), podemos tirar os termos de variação limitada e assim

$$
\llbracket \mathbf{H} \bullet \mathbf{X}, \mathbf{Y} \rrbracket=\llbracket \mathbf{H} \bullet \mathbf{M}, \mathbf{N} \rrbracket .
$$

Mas, pelo teorema 4.25 temos que $\llbracket \mathbf{H} \bullet \mathbf{M}, \mathbf{N} \rrbracket=\mathbf{H} \bullet \llbracket \mathbf{M}, \mathbf{N} \rrbracket$ e pela definição de covariação quadrática para semi-martingais* $\llbracket \mathbf{M}, \mathbf{N} \rrbracket=\llbracket \mathbf{X}, \mathbf{Y} \rrbracket$, assim, resulta que

$$
\llbracket \mathbf{H} \bullet \mathbf{X}, \mathbf{Y} \rrbracket=\mathbf{H} \bullet \llbracket \mathbf{M}, \mathbf{N} \rrbracket=\mathbf{H} \bullet \llbracket \mathbf{X}, \mathbf{Y} \rrbracket
$$

como queríamos.

Proposição 4.32. Sejam $\mathbf{X}$ um semi-martingal contínuo e $\mathbf{H}, \mathbf{H}^{\prime} \in \mathcal{L}(\mathbf{X})$. Se $\mathbf{H}$ e $\mathbf{H}^{\prime}$ forem indistinguíveis então $\mathbf{H} \bullet \mathbf{X}$ e $\mathbf{H}^{\prime} \bullet \mathbf{X}$ também o são.

Demonstração. Defina $\mathbf{Y} \doteq \mathbf{H}-\mathbf{H}^{\prime}$. Temos que as trajetórias $t \mapsto Y(t, \omega)$ são identicamente nulas para todo $\omega$ no complementar de um conjunto de medida P-nula. Devemos mostrar que $\mathbf{Y} \bullet \mathbf{X}$ é indistinguível do processo nulo. Pelo teorema 4.31, temos que $\mathbf{Y} \bullet \mathbf{X}$ é contínuo e portanto pela proposição 2.3 na página 6 , basta mostrar que $\mathbf{Y} \bullet \mathbf{X}$ é uma modificação do processo nulo, isto é, basta mostrar que para cada $t \geq 0,(\mathbf{Y} \bullet \mathbf{X})_{t}=0$, P-q.s.

Se $\mathbf{X}$ for um processo de variação finita, o resultado segue pela definição $\omega$-a- $\omega$ da integral estocástica para processos de variação finita. Agora, se $\mathbf{X}$ for um martingal local contínuo, então,

\footnotetext{
* Veja a definição 3.27 na página 57.
} 
pelo lema 4.27 temos

$$
\begin{aligned}
\llbracket \mathbf{Y} \bullet \mathbf{X} \rrbracket_{t} & =\int_{0}^{t} \mathbf{Y}^{2}(s, \omega) \llbracket \mathbf{X} \rrbracket(d s, \omega) \\
& =0
\end{aligned}
$$

pois $Y^{2}(\cdot, \omega)=0$, P-q. s. Segue que $\llbracket \mathbf{Y} \bullet \mathbf{X} \rrbracket=0$ e portanto, pela proposição 3.13 na página 47, item iii), segue que $\mathbf{Y} \bullet \mathbf{X}$ é constante e como $(\mathbf{Y} \bullet \mathbf{X})_{0}=0$, segue que $(\mathbf{Y} \bullet \mathbf{X})_{t}=0$, P-q. s. O caso geral, isto é, $\mathbf{X}$ um semi-martingal contínuo, resulta da decomposição semi-martingal de $\mathbf{X}$.

A propriedade associativa para este caso geral é um simples corolário dos teoremas 4.28 e 4.7 .

Teorema 4.33. Seja $\mathbf{X}$ um semi-martingal contínuo. Se $\mathbf{K} \in \mathcal{L}(\mathbf{X})$ e $\mathbf{H} \in \mathcal{L}(\mathbf{K} \bullet \mathbf{X})$ então $\mathbf{H K} \in$ $\mathcal{L}(\mathbf{X})$ e vale a propriedade associativa $\mathbf{H} \bullet(\mathbf{K} \bullet \mathbf{X})=(\mathbf{H K}) \bullet \mathbf{X}$.

\subsection{O teorema da convergência dominada estocástica}

Os resultados apresentados nesta seção serão cruciais para a demonstração da fórmula de Itô. Um dos resultados fundamentais do cálculo estocástico.

Dado $\mathbf{X}$ um semi-martingal contínuo, começaremos definindo um espaço de processos integrandos que não dependem de $\mathbf{X}$.

Definição 4.34. Denotamos por $\Lambda_{b}$ o espaço de todos os processos progressivamente mensuráveis localmente limitados, isto é, $\mathbf{H} \in \Lambda_{b}$ quando existir uma sequência $\left\{\tau_{n}\right\}_{n \geq 1}$ de tempos de parada tal que $\tau_{n} \uparrow \infty\left|H^{\tau_{n}}\right| \leq C_{n}<\infty$ para todo $(\omega, t) \in \mathbb{R}_{+} \times \Omega, n \geq 1$ e $C_{n}$ constantes.

Vamos também assumir que a $\sigma$-àlgebra $\mathscr{F}_{0}$ da filtração $\left\{\mathscr{F}_{t}\right\}_{t \geq 0}$ é a trivial (isto é, só é composta dos conjuntos de medida nula e seus complementares*.) Esta hipótese é intuitiva pois como consequência, para todo processo adaptado $\mathbf{X}$, a variável aleatória $X_{0}$ é constante P-q. s. ou seja, o valor de cada processo no instante zero (que pode ser pensado como o instante presente) é conhecida. Com essa hipótese, $X_{0}$ é integrável e podemos usar a definição alternativa (isto é, a definição 2.47 na página 27) de martingal local.

\footnotetext{
* Note que ela possivelmente é maior do que apenas $\{\Omega, \emptyset\}$ pois sob as condições usuais, a filtração é aumentada.
} 
Observamos também que se todas as trajetórias de um processo adaptado $\mathbf{H}$ forem contínuas, então $\mathbf{H}$ é localmente limitado. De fato, tome $\tau_{n} \doteq \inf \left\{t \geq 0:\left|H_{t}\right|>n\right\}$. Temos que $\left\{\tau_{n}\right\}_{n \geq 1}$ é uma sequência de tempos de parada, $\tau_{n} \uparrow \infty$ e $\left|H^{\tau_{n}}\right| \leq n$, para todo $n>\left|H_{0}\right|$.

O próximo resultado justifica que de fato, $\Lambda_{b}$ é um espaço de integrandos que não dependem do integrador $\mathbf{X}$.

Proposição 4.35. Seja $\mathbf{X}$ um semi-martingal contínuo. Então $\Lambda_{b} \subseteq \mathcal{L}(\mathbf{X})$.

Demonstração. Considere $\mathbf{X}=\mathbf{M}+\mathbf{A}$ e sejam $\mathbf{H} \in \Lambda_{b}$ e $\left\{\tau_{n}\right\}_{n \geq 1}$ sequência de tempos de parada tal que $\tau_{n} \uparrow \infty$ e $\left|H^{\tau_{n}}\right| \leq C_{n}<\infty$, para todo $n \geq 1$, onde cada $C_{n}$ é uma constante. Fixe $t \geq 0$ e $\omega \in \Omega$ tal que as trajetórias $s \mapsto A(s, \omega)$ sejam de variação finita nos intervalos finitos e denote por $V(t, \omega)$ a variação total dessa trajétoria no intervalo $[0, t]$. Temos que para todo $n \geq 1$,

$$
\int_{0}^{t \wedge \tau_{n}(\omega)}|H(s, \omega)| \mathrm{V}_{\mathbf{A}}(d s, \omega) \leq C_{n} V(t, \omega)<\infty .
$$

Escolhendo $n$ suficientemente grande, temos $\tau_{n}(\omega)>t$ e assim, temos P-q. s.

$$
\int_{0}^{t}|H(s, \omega)| \mathrm{V}_{\mathbf{A}}(d s, \omega)<\infty
$$

$\operatorname{logo}, \mathbf{H} \in \mathcal{L}_{\text {loc }}^{1}(\mathbf{A})$. Da mesma maneira, temos, para todo $n \geq 1$,

$$
\int_{0}^{t \wedge \tau_{n}(\omega)} H^{2}(s, \omega) \llbracket \mathbf{M} \rrbracket(d s, \omega) \leq C_{n}^{2} \llbracket \mathbf{M} \rrbracket(t, \omega)<\infty
$$

e como antes, isso mostra que, para quase todo $\omega \in \Omega$,

$$
\int_{0}^{t} H^{2}(s, \omega) \llbracket \mathbf{M} \rrbracket(d s, \omega)<\infty .
$$

Pela proposição 4.23, segue que $\mathbf{H} \in \mathcal{L}_{\text {loc }}^{2}(\mathbf{M})$, e portanto $\mathbf{H} \in \mathcal{L}(\mathbf{X})$, como queríamos.

O próximo resultado, mostra que a integral estocástica está de acordo com a intuição apresentada 
no início do capítulo.

Proposição 4.36. Sejam $\mathbf{X}$ um semi-martingal contínuo, $\sigma \leq \tau$ tempos de parada e $\xi$ uma variável aleatória $\mathscr{F}_{\sigma}$-mensurável. Então, $\mathbf{H}=\xi \mathbb{1}_{[] \sigma, \tau]]} \in \mathcal{L}(\mathbf{X})$ e $\mathbf{H} \bullet \mathbf{X}=\xi\left(\mathbf{X}^{\tau}-\mathbf{X}^{\sigma}\right)$.

Demonstração. Seja $\mathbf{X}=\mathbf{M}+\mathbf{A}$ a decomposição de $\mathbf{X}$. O plano será provar separadamente que $\mathbf{H} \in \mathcal{L}_{\text {loc }}^{1}(\mathbf{A})$ e $\mathbf{H} \bullet \mathbf{A}=\xi\left(\mathbf{A}^{\tau}-\mathbf{A}^{\sigma}\right)$ e que $\mathbf{H} \in \mathcal{L}_{\text {loc }}^{2}(\mathbf{M})$ e $\mathbf{H} \bullet \mathbf{M}=\xi\left(\mathbf{M}^{\tau}-\mathbf{M}^{\sigma}\right)$. Assim, estará provado o resultado pois

$$
\begin{aligned}
\mathbf{H} \bullet \mathbf{X} & =\mathbf{H} \bullet \mathbf{M}+\mathbf{H} \bullet \mathbf{A} \\
& =\xi\left(\mathbf{M}^{\tau}-\mathbf{M}^{\sigma}\right)+\xi\left(\mathbf{A}^{\tau}-\mathbf{A}^{\sigma}\right) \\
& =\xi\left((\mathbf{M}+\mathbf{A})^{\tau}-(\mathbf{M}+\mathbf{A})^{\sigma}\right) \\
& =\xi\left(\mathbf{X}^{\tau}-\mathbf{X}^{\sigma}\right) .
\end{aligned}
$$

Para a primeira parte do plano, seja $t \geq 0$ arbitrário. Temos que

$$
\begin{aligned}
\int_{0}^{t}|H(s, \omega)| \mathrm{V}_{\mathbf{A}}(d s, \omega) & =|\xi(\omega)| \int_{0}^{t} \mathbb{1}_{[] \sigma, \tau]]}(s, \omega) \mathrm{V}_{\mathbf{A}}(d s, \omega) \\
& =|\xi(\omega)|\left[\int_{0}^{t} \mathbb{1}_{[[0, \tau]]}(s, \omega) \mathrm{V}_{\mathbf{A}}(d s, \omega)-\int_{0}^{t} \mathbb{1}_{[[0, \sigma]]}(s, \omega) \mathrm{V}_{\mathbf{A}}(d s, \omega)\right] \\
& =|\xi(\omega)|\left[\mathrm{V}_{\mathbf{A}}(t \wedge \tau, \omega)-\mathrm{V}_{\mathbf{A}}(t \wedge \sigma, \omega)\right]<\infty
\end{aligned}
$$

e portanto $\mathbf{H} \in \mathcal{L}_{\text {loc }}^{1}(\mathbf{A})$. Afirmamos que para terminar a demonstração desta primeira parte, é suficiente mostrar que

$$
\mathbf{A}^{\tau}=A_{0}+\mathbf{A} \bullet \mathbb{1}_{[[0, \tau]]}
$$

De fato, se isto valer, então

$$
\begin{aligned}
\xi \mathbb{1}_{[] \sigma, \tau]]} \bullet \mathbf{A} & =\xi\left[\left(\mathbb{1}_{[[0, \tau]]}-\mathbb{1}_{[[0, \sigma]]}\right) \bullet \mathbf{A}\right] \\
& =\xi\left[\mathbf{A} \bullet \mathbb{1}_{[[0, \tau]]}-\mathbf{A} \bullet \mathbb{1}_{[[0, \sigma]]}\right] \\
& =\xi\left[\mathbf{A}^{\tau}-A_{0}-\left(\mathbf{A}^{\sigma}-A_{0}\right)\right] \\
& =\xi\left(\mathbf{A}^{\tau}-\mathbf{A}^{\sigma}\right) .
\end{aligned}
$$


Vamos provar portanto que (4.16) vale. De fato, seja $t \geq 0$,

$$
\begin{aligned}
\left(A_{0}+\mathbf{A} \bullet \mathbb{1}_{[[0, \tau]]}\right)(t, \omega) & =A_{0}+\int_{0}^{t} \mathbb{1}_{[[0, \tau(\omega)]]}(s) \mathbf{A}(d s, \omega) \\
& =A_{0}+\int_{0}^{t \wedge \tau(\omega)} \mathbb{1}(s, \omega) \mathbf{A}(d s, \omega) \\
& =A_{0}+\left(A(t \wedge \tau(\omega), \omega)-A_{0}\right) \\
& =A^{\tau}(t, \omega) .
\end{aligned}
$$

Vamos agora tratar da segunda parte. Começaremos provando que $\mathbf{H} \in \mathcal{L}_{\text {loc }}^{2}(\mathbf{M})$. Para isto, vamos verificar que $\mathbf{H}$ é progressivamente mensurável. Seja $t \geq 0$ fixo. Temos que

$$
\left.\mathbf{H}\right|_{[0, t] \times \Omega}=\xi \mathbb{1}_{[[\sigma \wedge t, \tau \wedge t]]}=\xi \mathbb{1}_{[\sigma<t]} \mathbb{1}_{[] \sigma \wedge t, \tau \wedge t]]} \cdot
$$

Agora, como $\xi$ é $\mathscr{F}_{\sigma}$-mensurável, afirmamos que $\xi \mathbb{1}_{[\sigma<t]}$ é $\mathscr{F}_{t}$-mensurável. Vamos provar esta afirmação. Suponha inicialmente que $\xi=\mathbb{1}_{[F]}$ onde $F \in \mathscr{F}_{\sigma}$ e seja $t \geq 0$ arbitrário. Então, pela definição de $\mathscr{F}_{\sigma}$, temos que $F \cap[\tau<t] \in \mathscr{F}_{t}$, de forma equivalente, $\xi \mathbb{1}_{[\sigma<t]}=\mathbb{1}_{[F \cap[\sigma<t]]}$ é $\mathscr{F}_{t^{-}}$ mensurável. Quando $\xi$ for uma função simples, segue por linearidade que $\xi \mathbb{1}_{[\sigma<t]}$ é $\mathscr{F}_{t}$-mensurável. Agora, suponha $\xi$ não negativa, $\mathscr{F}_{\sigma}$-mensurável e $t \geq 0$ e tome uma sequência $\left\{\xi_{n}\right\}_{n \geq 1}$ de funções simples não negativas e $\mathscr{F}_{\sigma}$-mensuráveis tal que $\xi_{n} \uparrow \xi$. Então $\xi_{n} \mathbb{1}_{[\sigma<t]} \uparrow \xi \mathbb{1}_{[\sigma<t]}$. Temos que $\xi_{n} \mathbb{1}_{[\sigma<t]}$ é $\mathscr{F}_{t}$-mensurável para cada $n \geq 1$ e portanto $\xi \mathbb{1}_{[\sigma<t]}$ é $\mathscr{F}_{t}$-mensurável. Finalmente, quando $\xi$ for arbitrário e $\mathscr{F}_{\sigma}$-mensurável, basta considerar a decomposição $\xi=\xi^{+}-\xi^{-}$.

Voltando, temos então que $\xi \mathbb{1}_{[\sigma<t]}$ é $\mathscr{F}_{t}$-mensurável. Como os tempos de parada $\sigma \wedge t, \tau \wedge t: \Omega \rightarrow$ $[0, t]$ são $\mathscr{F}_{t}$-mensuráveis, o intervalo estocástico $\left.] \sigma \wedge t, \tau \wedge t\right]$ é $\sigma\left(\mathscr{B}_{[0, t]} \times \mathscr{F}_{t}\right)$-mensurável. De fato,

$$
\begin{aligned}
] \sigma \wedge t, \tau \wedge t] & \doteq\{(s, \omega) \in[0, t] \times \Omega: \sigma(\omega) \wedge t<s \leq \tau(\omega) \wedge t\} \\
& =\{(s, \omega) \in[0, t] \times \Omega: \tau(\omega)-t-s \geq 0\} \cap\{(s, \omega) \in[0, t] \times \Omega: s-\tau(\omega) \wedge t>0\} .
\end{aligned}
$$

Disso segue que $\left.\mathbf{H}\right|_{[0, t] \times \Omega}$ é $\sigma\left(\mathscr{B}_{[0, t]} \times \mathscr{F}_{t}\right)$-mensurável, ou seja, $\mathbf{H}$ é progressivamente mensurável. 
Agora,

$$
\begin{aligned}
\int_{0}^{t} H^{2}(s, \omega) \llbracket \mathbf{M} \rrbracket(d s, \omega) & =\xi^{2}(\omega) \int_{0}^{t} \mathbb{1}_{\llbracket \sigma, \tau] \rrbracket} \llbracket \mathbf{M} \rrbracket(d s, \omega) \\
& =\xi^{2}(\omega)(\llbracket \mathbf{M} \rrbracket(t \wedge \tau(\omega), \omega)-\llbracket \mathbf{M} \rrbracket(t \wedge \sigma(\omega), \omega)) \\
& <\infty \quad \mathrm{P}-\text { q.s. }
\end{aligned}
$$

e portanto, pela proposição 4.23 temos que $\mathbf{H} \in \mathcal{L}_{\text {loc }}^{2}(\mathbf{M})$. Resta agora provar que $\mathbf{H} \bullet \mathbf{M}=$ $\xi\left(\mathbf{M}^{\tau}-\mathbf{M}^{\sigma}\right)$. Para isto, defina $\mathbf{Y} \doteq \xi\left(\mathbf{M}^{\tau}-\mathbf{M}^{\sigma}\right)$. Temos que $Y_{0}=0$. Vamos mostrar que $\mathbf{Y}$ é um martingal local contínuo. Note que podemos escrever $Y_{t}=\xi \mathbb{1}_{[\sigma<t]}\left(M_{t \wedge \tau}-M_{t \wedge \sigma}\right)$. Como $\xi \mathbb{1}_{[\sigma<t]}$ é $\mathscr{F}_{t}$-mensurável, temos que $\mathbf{Y}$ é adaptado e evidentemente contínua. Pela proposição 2.52 na página 30, podemos tomar uma sequência de tempos de parada $\left\{\rho_{n}\right\}_{n \geq 1}$ tal que $\mathbf{M}^{\tau \wedge \rho_{n}}$ e $\mathbf{M}^{\sigma \wedge \rho_{n}}$ sejam uniformemente limitados. Dessa forma $\mathbf{Y}$ é uniformemente limitado (por ser diferença de processos uniformemente limitados) e os processos $\mathbf{M}^{\tau \wedge \rho_{n}}$ e $\mathbf{M}^{\sigma \wedge \rho_{n}}$ são, para cada $n$, martingais (pelo corolário 2.56 na página 31). Agora, basta mostrar que para cada $n \geq 1$, $\mathbf{Y}^{\rho_{n}}$ é um martingal, pois isso implica que $\left\{\rho_{n}\right\}_{n}$ é uma sequência redutora o que por sua vez implica que satisfizemos as condições de martingal local pela definição. Para isto, seja $\lambda$ um tempo de parada limitado. Pelo teorema 2.37 na página 21 , basta verificar que

$$
\mathbf{E}\left(Y^{\rho_{n}}(\lambda)\right)=\mathbf{E}\left(Y^{\rho_{n}}(0)\right)=\mathbf{E}(Y(0))=0
$$

De fato,

$$
\begin{aligned}
\mathbf{E}\left(Y^{\rho_{n}}(\lambda)\right) & =\mathbf{E}\left(\mathbf{E}\left(Y^{\rho_{n}}(\lambda) \| \mathscr{F}_{\sigma}\right)\right) \\
& =\mathbf{E}\left(\mathbf{E}\left(\xi\left(\rho_{n}(\omega)\right)\left(M_{\tau \wedge \lambda}-M_{\sigma \wedge \lambda}\right)^{\rho_{n}} \| \mathscr{F}_{\sigma}\right)\right) \\
& =\mathbf{E}\left(\xi\left(\rho_{n}(\omega)\right) \mathbf{E}\left(M_{\tau \wedge \lambda}^{\rho_{n}}-M_{\sigma \wedge \lambda}^{\rho_{n}} \| \mathscr{F}_{\sigma}\right)\right) \\
& =\mathbf{E}\left(\xi\left(\rho_{n}(\omega)\right)\left(\mathbf{E}\left(M_{\tau \wedge \lambda}^{\rho_{n}} \| \mathscr{F}_{\sigma}\right)-\mathbf{E}\left(M_{\sigma \wedge \lambda}^{\rho_{n}} \| \mathscr{F}_{\sigma}\right)\right)\right) \\
& =\mathbf{E}\left(\xi\left(\rho_{n}(\omega)\right)\left(M_{\lambda \wedge \sigma}^{\rho_{n}}-M_{\lambda \wedge \sigma}^{\rho_{n}}\right)\right) \\
& =0 .
\end{aligned}
$$

Agora, falta provar que $\llbracket \xi\left(\mathbf{M}^{\tau}-\mathbf{M}^{\sigma}\right), \mathbf{N} \rrbracket=\mathbf{H} \bullet \llbracket \mathbf{M}, \mathbf{N} \rrbracket$ para todo martingal contínuo $\mathbf{N}$. Pela 
proposição 3.29 na página 58, temos

$$
\begin{aligned}
\llbracket \xi\left(\mathbf{M}^{\tau}-\mathbf{M}^{\sigma}\right), \mathbf{N} \rrbracket & =\xi \llbracket \mathbf{M}^{\tau}-\mathbf{M}^{\sigma}, \mathbf{N} \rrbracket \\
& =\xi\left(\llbracket \mathbf{M}, \mathbf{N} \rrbracket^{\tau}-\llbracket \mathbf{M}, \mathbf{N} \rrbracket^{\sigma}\right) \\
& =\xi\left(\llbracket \mathbf{M}, \mathbf{N} \rrbracket_{0}+\mathbb{1}_{[[0, \tau]]} \bullet \llbracket \mathbf{M}, \mathbf{N} \rrbracket-\llbracket \mathbf{M}, \mathbf{N} \rrbracket_{0}-\mathbb{1}_{[[0, \sigma]]} \bullet \llbracket \mathbf{M}, \mathbf{N} \rrbracket\right) \\
& =\xi\left(\mathbb{1}_{[[0, \tau]]} \bullet \llbracket \mathbf{M}, \mathbf{N} \rrbracket-\mathbb{1}_{[[0, \sigma]]} \bullet \llbracket \mathbf{M}, \mathbf{N} \rrbracket\right) \\
& =\xi\left(\left(\mathbb{1}_{[[0, \tau]]}-\mathbb{1}_{[[0, \sigma]]} \bullet \llbracket \mathbf{M}, \mathbf{N} \rrbracket\right)\right. \\
& =\xi \mathbb{1}_{[\rrbracket \sigma, \tau]]} \bullet \llbracket \mathbf{M}, \mathbf{N} \rrbracket \\
& =\mathbf{H} \bullet \llbracket \mathbf{M}, \mathbf{N} \rrbracket
\end{aligned}
$$

Agora vamos apresentar o análogo do teorema da convergência dominada para o caso estocástico.

Teorema 4.37. Sejam $\mathbf{X}$ um semi-martingal contínuo e $\left\{\mathbf{H}_{n}\right\}_{n \geq 1}$ uma sequência de processos em $\Lambda_{b}$ satisfazendo $\mathbf{H}_{n} \longrightarrow 0$, para todo $(t, \omega) \in \mathbb{R}_{+} \times \Omega$. Se existir um processo $\mathbf{K} \in \Lambda_{b}$ tal que $\left|\mathbf{H}_{n}\right| \leq \mathbf{K}$, para todo $n \geq 1$, então $\left(\mathbf{H}_{n} \bullet \mathbf{X}\right) \stackrel{\mathrm{P}}{\rightarrow} 0$, quando $n \uparrow \infty$, para todo $t \geq 0$.

Demonstração. Observamos que o resultado continuaria valendo se a desigualdade $\left|\mathbf{H}_{n}\right| \leq \mathbf{K}$ valesse apenas para $\omega$ no complementar de um conjunto de medida P-nulo $E$. De fato, bastaria trocar $\mathbf{H}_{n}$ por $\mathbf{H}_{n}^{\prime}=\mathbf{H}_{n} \mathbb{1}_{[0, \infty] \times E]}$, pois $\mathbf{H}$ e $\mathbf{H}^{\prime}$ são indistinguíveis e o resultado segue pela proposição 4.32 .

Vamos separar a demonstração primeiro tratando $\mathbf{X}$ como um processo de variação finita e depois como martingal local contínuo. Por hipótese, para cada $\omega \in \Omega$ e todo $n \geq 1, H_{n}(\cdot, \omega)$ é integrável em todo intervalo $[0, t]$. Pelo teorema da convergência dominada clássico, temos que para $s \leq t \mathrm{e}$ $n \rightarrow \infty$,

$$
\left|\int_{0}^{s} \mathbf{H}_{n} d \mathbf{X}-0\right| \leq \int_{0}^{t}\left|\mathbf{H}_{n}\right| d \mathrm{~V}_{\mathbf{X}} \longrightarrow 0 .
$$

Assim, pontualmente temos, para qualquer $t \geq 0$,

$$
\sup _{s \leq t}\left|\left(\mathbf{H}_{n} \bullet \mathbf{X}\right)(s)\right| \longrightarrow 0
$$

Como em espaços de medida finita, convergência pontual implica na convergência em medida, isto é, 
em probabilidade, provamos o caso quando o integrador é de variação finita.

Agora, seja X um martingal local contínuo. Pela definição do espaço $\Lambda_{b}$ e pela proposição 2.52 na página 30, podemos tomar uma sequência de tempos de parada $\left\{\tau_{m}\right\}_{m \geq 1}$ tal que $\tau_{m} \uparrow \infty,\left|\mathbf{K}^{\tau_{m}}\right| \leq$ $C_{m}<\infty$ e $\mathbf{X}^{\tau_{m}} \in \mathrm{cH}^{2}$, onde $\left\{C_{m}\right\}_{m \geq 1}$ são constantes.

Fixe $m \geq 1$ e seja $t \geq 0$, arbitrário. Vamos provar que $\left(\mathbf{H}_{n} \bullet \mathbf{X}\right)(t, \cdot) \longrightarrow 0$ em probabilidade no conjunto $\left[\tau_{m} \geq t\right]$. Da arbitráriedade de $m$ e como $\cup_{m}\left[\tau_{m} \geq t\right]=\Omega$, resultará pela proposição B.3 na página 154 , que $\left(\mathbf{H}_{n} \bullet \mathbf{X}\right)(t, \cdot) \longrightarrow 0$ em probabilidade em todo $\Omega$. Para isto, note inicialmente que $\mathbf{K}^{\tau_{m}} \in \mathcal{L}^{2}\left(\mathbf{X}^{\tau_{m}}\right) \doteq \mathcal{L}^{2}\left(\mathbb{R}_{+} \times \Omega, \mathfrak{P}_{g}, \alpha_{\mathbf{X}^{\tau_{m}}}\right)$. De fato,

$$
\begin{aligned}
\left\|\mathbf{K}^{\tau_{m}}\right\|_{\mathbf{X}^{\tau_{m}}} & =\mathbf{E}\left(\int_{0}^{\infty}\left(\mathbf{K}^{\tau_{m}}(s, \omega)\right)^{2} \llbracket \mathbf{X}^{\tau \mathbf{m}} \rrbracket(d s, \omega)\right) \\
& \leq C_{m}^{2} \mathbf{E}\left(\int_{0}^{\infty} \llbracket \mathbf{X}^{\tau_{\mathbf{m}}} \rrbracket(d s, \omega)\right) \\
& =C_{m}^{2} \mathbf{E}\left(\lim _{t \rightarrow \infty} \int_{0}^{t} \llbracket \mathbf{X}^{\tau_{\mathbf{m}}} \rrbracket(d s, \omega)\right) \\
& =C_{m}^{2} \mathbf{E}\left(\lim _{t \rightarrow \infty} \llbracket \mathbf{X}^{\tau_{\mathbf{m}}} \rrbracket(t, \cdot)-\llbracket \mathbf{X}^{\tau_{\mathbf{m}}} \rrbracket(0, \cdot)\right) \\
& =C_{m}^{2} \mathbf{E}\left(\lim _{t \rightarrow \infty} \llbracket \mathbf{X}^{\tau_{\mathbf{m}}} \rrbracket(t, \cdot)\right) .
\end{aligned}
$$

Como $\mathbf{X}^{\tau_{m}} \in \mathrm{cH}^{2}$, pela proposição 3.21 na página 53 , temos que $\mathbf{E}\left(\lim _{t \rightarrow \infty} \llbracket \mathbf{X}^{\tau_{\mathbf{m}}} \rrbracket(t, \cdot)\right)<\infty$ e portanto $\left\|\mathbf{K}^{\tau_{m}}\right\|_{\mathbf{X}^{\tau_{m}}}<\infty$. Agora, por hipótese, $\left|\mathbf{H}_{n}^{\tau_{m}}\right| \leq\left|\mathbf{K}^{\tau_{m}}\right|$ e $\lim _{n \rightarrow \infty} \mathbf{H}_{n}^{\tau_{m}}(t, \omega)=0, \forall(t, \omega) \in$ $\mathbb{R}_{+} \times \Omega$. Segue pelo teorema da convergência dominada clássico que

$$
\begin{aligned}
\lim _{n \rightarrow \infty} \int_{\mathbb{R}_{+} \times \Omega}\left(H_{n}^{\tau_{m}}(s, \omega)\right)^{2} d \alpha_{\mathbf{X}^{\tau_{m}}} & =\int_{\mathbb{R}_{+} \times \Omega}\left(\lim _{n \rightarrow \infty} H_{n}^{\tau_{m}}(s, \omega)\right)^{2} d \alpha_{\mathbf{X}^{\tau_{m}}} \\
& =0
\end{aligned}
$$

ou seja, $\lim _{n \rightarrow \infty}\left\|\mathbf{H}_{n}^{\tau_{m}}\right\|_{\mathbf{X}^{\tau_{m}}}^{2}=0$. Agora, como $\left|H_{n}^{\tau_{m}}\right| \leq\left|\mathbf{K}^{\tau_{m}}\right|$ e $\mathbf{K}^{\tau_{m}} \in \mathcal{L}^{2}\left(\mathbf{X}^{\tau_{m}}\right)$, temos que $\mathbf{H}_{n}^{\tau_{m}} \in$ $\mathcal{L}^{2}\left(\mathbf{X}^{\tau_{m}}\right)$. Além disso, lembre-se que $\mathbf{X}^{\tau_{m}} \in \mathrm{cH}^{2}$ e portanto estamos sob as hipóteses do teorema 4.17, $\operatorname{logo} \mathbf{H}_{n}^{\tau_{m}} \bullet \mathbf{X}^{\tau_{m}} \in \mathrm{cH}_{0}^{2}$ e pela isometria de Itô, $\left\|\mathbf{H}_{n}^{\tau_{m}}\right\|_{\mathbf{X}^{\tau_{m}}}^{2}=\left\|\mathbf{H}_{n}^{\tau_{m}} \bullet \mathbf{X}^{\tau_{m}}\right\|_{\mathcal{H}^{2}}^{2}$ para todo $n \geq 1$, e 
portanto, pela proposição 4.21

$$
\begin{aligned}
\lim _{n \rightarrow \infty}\left\|\mathbf{H}_{n}^{\tau_{m}} \bullet \mathbf{X}^{\tau_{m}}\right\|_{\mathcal{H}^{2}}^{2} & =\lim _{n \rightarrow \infty}\left\|\left(\mathbf{H}_{n} \bullet \mathbf{X}\right)^{\tau_{m}}\right\|_{\mathcal{H}^{2}}^{2} \\
& \doteq \lim _{n \rightarrow \infty}\left(\sup _{t \geq 0}\left\|\left(\mathbf{H}_{n} \bullet \mathbf{X}\right)\left(\tau_{m}(\cdot) \wedge t, \cdot\right)\right\|_{2}^{2}\right) \\
& =0
\end{aligned}
$$

Agora em $\left[\tau_{m} \geq t\right]$,

$$
0=\lim _{n \rightarrow \infty}\left(\sup _{t \geq 0}\left\|\left(\mathbf{H}_{n} \bullet \mathbf{X}\right)(t, \cdot)\right\|_{2}^{2}\right)=\lim _{n \rightarrow \infty}\left\|\mathbf{H}_{n} \bullet \mathbf{X}\right\|_{2}^{2}
$$

e pela desigualdade de Doob*, ficamos com

$$
\begin{aligned}
\left\|\sup _{t \geq 0}\left|\left(\mathbf{H}_{n} \bullet \mathbf{X}\right)(t, \cdot)\right|\right\|_{2}^{2} & \leq 4 \sup _{t \geq 0}\left\|\left(\mathbf{H}_{n} \bullet \mathbf{X}\right)(t, \cdot)\right\|_{2}^{2} \\
& \doteq 4\left\|\mathbf{H}_{n} \bullet \mathbf{X}\right\|_{\mathcal{H}^{2}}^{2}
\end{aligned}
$$

Dessa última igualdade e de (4.17), ficamos com

$$
\lim _{n \rightarrow \infty}\left\|\sup _{t \geq 0}\left|\left(\mathbf{H}_{n} \bullet \mathbf{X}\right)(t, \cdot)\right|\right\|_{2}^{2}=0 .
$$

Pela proposição B.2 na página 154, a convergência em $\mathrm{L}^{2}$ implica na convergência em probabilidade e portanto $\sup _{t \geq 0}\left|\left(\mathbf{H}_{n} \bullet \mathbf{X}\right)(t, \cdot)\right| \longrightarrow 0$, em probabilidade em $\left[\tau_{m} \geq t\right]$, e portanto pela proposição B.3 na página 154 , resulta o que queríamos.

Uma das principais consequências do teorema da convergência dominada estocástica é que podemos deixar precisa uma relação entre a integral estocástica e o conceito intuitivo de somas de Riemann. Para ver isto, considere $\mathbf{X}$ um semi-martingal contínuo e $\mathbf{H}$ um processo estocástico adaptado e contínuo quase-sempre. Fixe $t \geq 0$ e seja $\mathcal{P}: 0=t_{0}<t_{1}<\ldots<t_{n}=t$ uma partição de $[0, t]$. Defina a variável aleatória $S^{(\mathcal{P}, \mathbf{H}, \mathbf{X})}: \Omega \mapsto \mathbb{R}$ por

$$
S^{(\mathcal{P}, \mathbf{H}, \mathbf{X})}(\omega) \doteq \sum_{j=1}^{n} H\left(t_{j-1}, \omega\right)\left[X\left(t_{j}, \omega\right)-X\left(t_{j-1}, \omega\right)\right]
$$

\footnotetext{
* Veja o teorema 2.27 na página 18.
} 
Defina também o processo estocástico

$$
\mathbf{R}^{(\mathcal{P}, \mathbf{H})}(\cdot, \cdot) \doteq H(0, \cdot) \mathbb{1}_{[\{0\}]}(\cdot, \cdot)+\sum_{j=1}^{n} H\left(t_{j-1}, \cdot\right) \mathbb{1}_{\left.\left.[] t_{j-1}, t_{j}\right]\right]}(\cdot, \cdot) .
$$

Como para cada $j \in\{1,2, \ldots, n+1\}, H\left(t_{j-1}, \cdot\right)$, é uma variável aleatória $\mathscr{F}_{t_{j-1}}$-mensurável, temos pela proposição 4.36 que $H\left(t_{j-1}, \cdot\right) \mathbb{1}_{\left.\left.[] t_{j-1}, t_{j}\right]\right]}(\cdot, \cdot) \in \mathcal{L}(\mathbf{X})$ e

$$
\begin{aligned}
H\left(t_{j-1}, \cdot\right) \mathbb{1}_{\left.\left[t_{j-1}, t_{j}\right]\right]}(\cdot, \cdot) \bullet \mathbf{X}(\cdot, \cdot) & =H\left(t_{j-1}, \cdot\right)\left[X\left(t_{j} \wedge t, \cdot\right)-X\left(t_{j-1} \wedge t, \cdot\right)\right] \\
& =H\left(t_{j-1}, \cdot\right)\left[X\left(t_{j}, \cdot\right)-X\left(t_{j-1}, \cdot\right)\right]
\end{aligned}
$$

Assim, $\mathbf{R}^{(\mathcal{P}, \mathbf{H})} \in \mathcal{L}(\mathbf{X})$ e $\left(\mathbf{R}^{(\mathcal{P}, \mathbf{H})}\right)(t, \cdot)=S^{(\mathcal{P}, \mathbf{H}, \mathbf{X})}(\cdot)$, ou seja,

$$
\int_{0}^{t} R^{(\mathcal{P}, \mathbf{H})}(s, \cdot) \mathbf{X}(d s, \cdot)=\sum_{j=1}^{n} H\left(t_{j-1}, \cdot\right)\left[X\left(t_{j}, \cdot\right)-X\left(t_{j-1}, \cdot\right)\right] .
$$

Com essas definições em mente, temos o seguinte resultado:

Proposição 4.38. Sejam $\mathbf{X}$ um semi-martingal contínuo, $\mathbf{H}$ um processo quase sempre contínuo $e$ adaptado e $t \geq 0$. Então

$$
\int_{0}^{t} H(s, \cdot) \mathbf{X}(d s, \cdot)=\lim _{n \rightarrow \infty} S^{\left(\mathcal{P}_{n}, \mathbf{H}, \mathbf{X}\right)}(\cdot) \text { em probabilidade }
$$

para toda sequência $\left\{\mathcal{P}_{n}\right\}_{n \geq 1}$ de partições do intervalo $[0, t]$ tal que $\left|\mathcal{P}_{n}\right| \longrightarrow 0$ quando $n \uparrow \infty$.

Demonstração. Podemos supor sem perda de generalidade que $\mathbf{H}$ tem todas as trajetórias contínuas (de fato, podemos tomar $\mathbf{H}^{\prime}$ indistinguível de $\mathbf{H}$ e a integral estocástica é a mesma, veja a proposição 4.32). Dessa forma, temos que $\mathbf{H} \in \Lambda_{b}$ e portanto $\mathbf{H} \in \mathcal{L}(\mathbf{X})$.

Suponha inicialmente que $|\mathbf{H}| \leq C<\infty$, para alguma constante $C \geq 0$. Temos que

$$
\mathbf{R}^{\left(\mathcal{P}_{n}, \mathbf{H}\right)}(\cdot, \cdot) \doteq H(0, \cdot) \mathbb{1}_{[\{0\}]}(\cdot, \cdot)+\sum_{j=1}^{n} H\left(t_{j-1}, \cdot\right) \mathbb{1}_{\left.\left[t_{j-1}, t_{j}\right]\right]}(\cdot, \cdot) \longrightarrow \mathbf{H}(\cdot, \cdot)
$$

pontualmente, para todo $(t, \omega) \in \mathbb{R}_{+} \times \Omega$ e $\mathbf{R}^{\left(\mathcal{P}_{n}, \mathbf{H}\right)} \leq C$, para cada $n \geq 1$. Temos então que 
$\mathbf{R}^{\left(\mathcal{P}_{n}, \mathbf{H}\right)}-\mathbf{H} \longrightarrow 0$, pontualmente, para todo $(t, \omega) \in \mathbb{R}_{+} \times \Omega$ e $\left|\mathbf{R}^{\left(\mathcal{P}_{n}, \mathbf{H}\right)}-\mathbf{H}\right| \leq 2 C$. Temos pelo teorema da convergência dominada estocástica que $\left(\left(\mathbf{R}^{\left(\mathcal{P}_{n}, \mathbf{H}\right)}-\mathbf{H}\right) \bullet \mathbf{X}\right)_{t} \longrightarrow 0$, em probabilidade, ou seja

$$
\left(\mathbf{R}^{\left(\mathcal{P}_{n}, \mathbf{H}\right)} \bullet \mathbf{X}\right)_{t}=(\mathbf{H} \bullet \mathbf{X})_{t}=\lim _{n \rightarrow \infty} S^{\left(\mathcal{P}_{n}, \mathbf{H}, \mathbf{X}\right)}(\cdot)
$$

Agora, mais geralmente, temos que existe uma sequência $\left\{\tau_{m}\right\}_{m \geq 1}$ de tempos de parada tal que $\tau_{m} \uparrow \infty$ e $\left|\mathbf{H}^{\tau_{m}}\right| \leq C_{m}$, para todo $m>\left|H_{0}\right|^{*}$. Para este $m$, temos pelo caso anterior que

$$
S^{\left(\mathcal{P}_{n}, \mathbf{H}^{\tau_{m}}, \mathbf{X}\right)} \longrightarrow\left(\mathbf{H}^{\tau_{m}} \bullet \mathbf{X}\right)_{t}
$$

em probabilidade, para $n \uparrow \infty$. Assim, para $\omega \in\left[\tau_{m} \geq t\right]$, temos que $S^{\left(\mathcal{P}_{n}, \mathbf{H}^{\tau_{m}}, \mathbf{X}\right)}=S^{\left(\mathcal{P}_{n}, \mathbf{H}, \mathbf{X}\right)}$ e pelo teorema 4.31, temos que $\left(\mathbf{H}^{\tau_{m}} \bullet \mathbf{X}\right)_{t}=(\mathbf{H} \bullet \mathbf{X})_{t}^{\tau_{m}}=(\mathbf{H} \bullet \mathbf{X})_{t} . \operatorname{Logo}, S^{\left(\mathcal{P}_{n}, \mathbf{H}, \mathbf{X}\right)} \longrightarrow(\mathbf{H} \bullet \mathbf{X})_{t}$, em probabilidade, em cada $\left[\tau_{m} \geq t\right]$. Como $\cup_{m \geq\left|H_{0}\right|}\left[\tau_{m} \geq t\right]=\Omega$, o resultado segue da proposiçao B.3.

Corolário 4.39. Sejam $\mathbf{X}$ um semi-martingal contínuo, $\mathbf{H}$ um processo estocástico adaptado e quase sempre contínuo, $0 \leq a<b$ e $\xi$ uma variável aleatória $\mathscr{F}_{a}$-mensurável. Então, $\int_{a}^{b} \xi H(s, \cdot) \mathbf{X}(d s, \cdot)=\xi \int_{a}^{b} H(s, \cdot) \mathbf{X}(d s, \cdot)$.

Demonstração. Podemos assumir que $a=0$. Note que $\xi \mathbf{H}$ é q. s.-contínuo e adaptado e para cada partição $\mathcal{P}$ de $[a, b]$ vale a identidade $S^{(\mathcal{P}, \xi \mathbf{H}, \mathbf{X})}=\xi S^{(\mathcal{P}, \mathbf{H}, \mathbf{X})}$. O resultado segue da proposição anterior fazendo $|\mathcal{P}| \rightarrow 0$.

O próximo resultado pode ser pensado como a versão estocástica da fórmula de integração por partes.

Proposição 4.40. Sejam $\mathbf{X}$ e $\mathbf{Y}$ semi-martingais contínuos. Então para todo $t \geq 0$,

$$
X_{t} Y_{t}=X_{0} Y_{0}+\int_{0}^{t} X_{s} d Y_{s}+\int_{0}^{t} Y_{s} d X_{s}+\llbracket \mathbf{X}, \mathbf{Y} \rrbracket_{t} .
$$

Demonstração. Seja $\mathcal{P}: 0=t_{0}<t_{1}<\ldots<t_{n}=t$ uma partição arbitrária do intervalo $[0, t]$. Note a

\footnotetext{
* Lembre-se que $H_{0}$ é constante. Veja as observações após a definição 4.34.
} 
igualdade

$$
\begin{aligned}
X_{t_{j}} Y_{t_{j}}-X_{t_{j-1}} Y_{t_{j-1}} & =\left(X_{t_{j}}-X_{t_{j-1}}\right)\left(Y_{t_{j}}-Y_{t_{j-1}}\right)+X_{t_{j-1}}\left(Y_{t_{j}}-Y_{t_{j-1}}\right) \\
& +Y_{t_{j-1}}\left(X_{t_{j}}-X_{t_{j-1}}\right)
\end{aligned}
$$

e some sobre $j=1,2, \ldots, n$. Obtemos*

$$
X_{t} Y_{t}-X_{0} Y_{0}=Q^{(\mathcal{P}, \mathbf{X}, \mathbf{Y})}+S^{(\mathcal{P}, \mathbf{X}, \mathbf{Y})}+S^{(\mathcal{P}, \mathbf{Y}, \mathbf{X})} .
$$

Faça $|\mathcal{P}| \rightarrow 0$ e obtemos o resultado pelo teorema da convergência dominada estocástico e pela proposição 3.28 na página 57 .

Agora, vamos obter alguns resultados para integrais estocásticas com respeito ao processo de covariação quadrática $\llbracket \mathbf{X}, \mathbf{Y} \rrbracket$. Novamente, seja $\mathcal{P}: 0=t_{0}<t_{1}<\ldots<t_{n}=t$ uma partição do intervalo $[0, t]$. Defina a variável aleatória $S Q^{(\mathcal{P}, \mathbf{H}, \mathbf{X}, \mathbf{Y})}: \Omega \mapsto \mathbb{R}$ por

$$
S Q^{(\mathcal{P}, \mathbf{H}, \mathbf{X}, \mathbf{Y})}(\omega) \doteq \sum_{j=1}^{n} H\left(t_{j-1}, \omega\right)\left[\left(X\left(t_{j}, \omega\right)-X\left(t_{j-1}, \omega\right)\right)\left(Y\left(t_{j}, \omega\right)-Y\left(t_{j-1}, \omega\right)\right)\right]
$$

e vamos definir $S Q^{(\mathcal{P}, \mathbf{H}, \mathbf{X})} \doteq S Q^{(\mathcal{P}, \mathbf{H}, \mathbf{X}, \mathbf{X})}$. Observe também que da relação

$$
\begin{aligned}
4\left(X_{t_{j}}-X_{t_{j-1}}\right)\left(Y_{t_{j}}-Y_{t_{j-1}}\right) & =\left(\left(X_{t_{j}}+Y_{t_{j}}\right)-\left(X_{t_{j-1}}+Y_{t_{j-1}}\right)\right)^{2} \\
& -\left(\left(X_{t_{j}}-Y_{t_{j}}\right)-\left(X_{t_{j-1}}-Y_{t_{j-1}}\right)\right)^{2}
\end{aligned}
$$

segue que

$$
S Q^{(\mathcal{P}, \mathbf{H}, \mathbf{X}, \mathbf{Y})}=\frac{1}{4}\left[S Q^{(\mathcal{P}, \mathbf{X}+\mathbf{Y})}-S Q^{(\mathcal{P}, \mathbf{X}-\mathbf{Y})}\right] .
$$

Proposição 4.41. Sejam $\mathbf{X}$ um semi-martingal contínuo e $\mathbf{H}$ um processo estocástico adaptado, q.s.-contínuo e $t \geq 0$. Então $S Q^{\left(\mathcal{P}_{n}, \mathbf{H}, \mathbf{X}\right)} \longrightarrow \int_{0}^{t} H_{s} d \llbracket \mathbf{X} \rrbracket_{s}$ em probabilidade, para qualquer sequência de partições $\left\{\mathcal{P}_{n}\right\}_{n \geq 1}$ do intervalo $[0, t]$ tal que $\left|\mathcal{P}_{n}\right| \rightarrow 0$.

Demonstração. Por um argumento semelhante feito na demonstração da proposição 4.38, podemos assumir que as trajetórias de $\mathbf{H}$ e $\mathbf{X}$ são contínuas para todo $\omega \in \Omega$ e que $\mathbf{X}$ e $\mathbf{H}$ são limitados.

\footnotetext{
* Lembre-se da notação $Q^{(\mathcal{P}, \mathbf{X}, \mathbf{Y})}$ na proposição 3.18 na página 51.
} 
Agora, pela proposição 4.40 com $\mathbf{X}=\mathbf{Y}$, temos

$$
X_{t}^{2}-X_{0}^{2}=2 \int_{0}^{t} X_{s} d X_{s}+\llbracket \mathbf{X} \rrbracket_{t}
$$

Assim, se $\mathcal{P}: 0=t_{0}<t_{1}<\ldots<t_{n}=t$ é uma partição de $[0, t]$, temos para $1 \leq j \leq n$,

$$
X_{t_{j}}^{2}-X_{t_{j-1}}^{2}=2 \int_{t_{j-1}}^{t_{j}} X_{s} d X_{s}+\left(\llbracket \mathbf{X} \rrbracket_{t_{j}}-\llbracket \mathbf{X} \rrbracket_{t_{j-1}}\right)
$$

e disso, temos que

$$
\begin{aligned}
\left(X_{t_{j}}-X_{t_{j-1}}\right)^{2} & =\left(X_{t_{j}}^{2}-X_{t_{j-1}}^{2}\right)-2 X_{t_{j-1}}\left(X_{t_{j}}-X_{t_{j-1}}\right) \\
& =2 \int_{t_{j-1}}^{t_{j}} X_{s} d X_{s}+\left(\llbracket \mathbf{X} \rrbracket_{t_{j}}-\llbracket \mathbf{X} \rrbracket_{t_{j-1}}\right)-2 X_{t_{j-1}}\left(X_{t_{j}}-X_{t_{j-1}}\right) .
\end{aligned}
$$

Agora, pelo corolário 4.39,

$$
H_{t_{j-1}} \int_{t_{j-1}}^{t_{j}} X_{s} d X_{s}=\int_{t_{j-1}}^{t_{j}} H_{t_{j-1}} X_{s} d X_{s}=\int_{0}^{t} H_{t_{j-1}} \mathbb{1}_{\left.\left.[] t_{j-1}, t_{j}\right]\right]}(s) X_{s} d X_{s}
$$

Assim, multiplicando (4.19) por $H_{t_{j-1}}$, usando (4.20) e somando para $j=1,2, \ldots, n$ obtemos

$$
S Q^{(\mathcal{P}, \mathbf{H}, \mathbf{X})}=2 \int_{0}^{t} R_{s}^{(\mathcal{P}, \mathbf{H})} X_{s} d X_{s}+S^{(\mathcal{P}, \mathbf{H}, \llbracket \mathbf{X} \rrbracket)}-2 S^{(\mathcal{P}, \mathbf{H X}, \mathbf{X})} .
$$

Agora faça $|\mathcal{P}| \rightarrow 0$. Por continuidade, $\mathbf{R}^{(\mathcal{P}, \mathbf{H})} \mathbf{X} \longrightarrow \mathbf{H X}$, pontualmente em $\mathbb{R}_{+} \times \Omega$ e o teorema da convergência dominada estocástica garante que

$$
\int_{0}^{t} R_{s}^{(\mathcal{P}, \mathbf{H})} X_{s} d X_{s} \stackrel{\mathrm{P}}{\rightarrow} \int_{0}^{t} H_{s} X_{s} d X_{s}
$$


Além disso, pela proposição 4.38 temos que

$$
S^{(\mathcal{P}, \mathbf{H X}, \mathbf{X})} \stackrel{\mathrm{P}}{\rightarrow} \int_{0}^{t} H_{s} X_{s} d X_{s} \text { e } S^{(\mathcal{P}, \mathbf{H}, \llbracket \mathbf{X} \rrbracket)} \stackrel{\mathrm{P}}{\rightarrow} \int_{0}^{t} H_{s} d \llbracket \mathbf{X} \rrbracket_{s} .
$$

Logo, de (4.22) e (4.23), temos por (4.21)

$$
S Q^{(\mathcal{P}, \mathbf{H}, \mathbf{X})} \stackrel{\mathrm{P}}{\rightarrow} \int_{0}^{t} H_{s} d \llbracket \mathbf{X} \rrbracket_{s},
$$

como queríamos.

Corolário 4.42. Sejam $\mathbf{Y}$ um semi-martingal contínuo e $\mathbf{X}, \mathbf{H}$ como na proposição anterior. Então $S Q^{\left(\mathcal{P}_{n}, \mathbf{H}, \mathbf{X}, \mathbf{Y}\right)} \longrightarrow \int_{0}^{t} H_{s} d \llbracket \mathbf{X}, \mathbf{Y} \rrbracket_{s}$ em probabilidade, para qualquer sequência de partições $\left\{\mathcal{P}_{n}\right\}_{n \geq 1}$ do intervalo $[0, t]$ tal que $\left|\mathcal{P}_{n}\right| \rightarrow 0$.

Demonstração. Pela relação (4.18) e pela proposição anterior, temos que

$$
S Q^{\left(\mathcal{P}_{n}, \mathbf{H}, \mathbf{X}, \mathbf{Y}\right)} \stackrel{\mathrm{P}}{\rightarrow} 4\left(\int_{0}^{t} H_{s} d \llbracket \mathbf{X}+\mathbf{Y} \rrbracket_{s}-\int_{0}^{t} H_{s} d \llbracket \mathbf{X}-\mathbf{Y} \rrbracket_{s}\right) .
$$

Agora, pela bilinearidade da integral estocástica* vem que

$$
\begin{aligned}
4\left(\int_{0}^{t} H_{s} d \llbracket \mathbf{X}+\mathbf{Y} \rrbracket_{s}-\int_{0}^{t} H_{s} d \llbracket \mathbf{X}-\mathbf{Y} \rrbracket_{s}\right) & =4((\mathbf{H} \bullet \llbracket \mathbf{X}+\mathbf{Y} \rrbracket)-(\mathbf{H} \bullet \llbracket \mathbf{X}-\mathbf{Y} \rrbracket)) \\
& =4(\mathbf{H} \bullet(\llbracket \mathbf{X}+\mathbf{Y} \rrbracket-\llbracket \mathbf{X}-\mathbf{Y} \rrbracket)) \\
& =\mathbf{H} \bullet(4(\llbracket \mathbf{X}+\mathbf{Y} \rrbracket-\llbracket \mathbf{X}-\mathbf{Y} \rrbracket)) \\
& =\mathbf{H} \bullet \llbracket \mathbf{X}, \mathbf{Y} \rrbracket
\end{aligned}
$$

Para obter a última igualdade usamos simplesmente a definição de co-variação quadrática ${ }^{\dagger}$. Substituindo (4.25) em (4.24), provamos o que queríamos.

\footnotetext{
* Veja o teorema 4.31. † Veja a definição 3.15 na página 50.
} 


\subsection{Integração com respeito a semi-martingais vetoriais}

Nessa seção, vamos ver resumidamente, como ficam os conceitos que desenvolvemos até agora no contexto vetorial. Dizemos que $\mathbf{X}=\left(\mathbf{X}^{1}, \ldots, \mathbf{X}^{d}\right)$ é semi-martingal contínuo d-dimensional quando cada componente $\mathbf{X}^{i}$ for um semi-martingal contínuo. Denotaremos por $\mathcal{S}^{d}$ a família de todos os semi-martingais contínuos $d$-dimensionais definidos em $\left(\Omega, \mathscr{F},\left\{\mathscr{F}_{t}\right\}, \mathrm{P}\right)$.

Definimos a variação quadrática de $\mathbf{X} \in \mathcal{S}^{d}$ pela fórmula $\llbracket \mathbf{X} \rrbracket \doteq \sum_{j=1}^{d} \llbracket \mathbf{X}^{j} \rrbracket$. De forma análoga ao caso escalar, se $\mathbf{X}$ é um martingal local ( $d$-dimensional), então $\llbracket \mathbf{X} \rrbracket$ é o único processo contínuo, de variação finita e que se anula em $t=0$ tal que $\|\mathbf{X}\|^{2}-\llbracket \mathbf{X} \rrbracket$ é um martingal local. Para $\mathbf{X}, \mathbf{Y} \in \mathcal{S}^{d}$ definimos o processo de covariação $\llbracket \mathbf{X}, \mathbf{Y} \rrbracket$ por $\llbracket \mathbf{X}, \mathbf{Y} \rrbracket \doteq \sum_{j=1}^{d} \llbracket \mathbf{X}^{j}, \mathbf{Y}^{j} \rrbracket \cdot \llbracket \mathbf{X}, \mathbf{Y} \rrbracket$ é o único processo contínuo, de variação finita e que se anula em $t=0$ tal que $\mathbf{X Y}-\llbracket \mathbf{X}, \mathbf{Y} \rrbracket$ é um martingal local.

Seja $\mathbf{Z}$ um semi-martingal contínuo escalar e $\mathbf{X} \in \mathcal{S}^{d}$. Definimos o produto $\mathbf{Z X} \doteq\left(\mathbf{Z X}^{1}, \mathbf{Z X}^{2}, \ldots, \mathbf{Z X}^{d}\right)^{\prime}$ e a covariação $\llbracket \mathbf{Z}, \mathbf{X} \rrbracket \doteq\left(\llbracket \mathbf{Z}, \mathbf{X}^{\mathbf{1}} \rrbracket, \llbracket \mathbf{Z}, \mathbf{X}^{\mathbf{2}} \rrbracket, \ldots, \llbracket \mathbf{Z}, \mathbf{X}^{d} \rrbracket\right)^{\prime}$.

Definimos os espaço $\mathcal{L}(\mathbf{X})$ dos processos integráveis com respeito a $\mathbf{X} \in \mathcal{S}^{d}$ como o espaço de todos os processos $\mathbf{H}=\left(\mathbf{H}^{1}, \mathbf{H}^{2}, \ldots, \mathbf{H}^{d}\right)^{\prime}$ com valores em $\mathbb{R}^{d}$, progressivamente mensuráveis tais que $\mathbf{H}^{j} \in \mathcal{L}\left(\mathbf{X}^{j}\right)$, para todo $j=1, \ldots, d$. Em outras palavras, sendo $\mathbf{X}^{j}=\mathbf{M}^{j}+\mathbf{A}^{j}$ a decomposição semi-martingal de cada componente de $\mathbf{X}$, temos que $\mathbf{H} \in \mathcal{L}(\mathbf{X})$ se, e somente se,

$$
\sum_{j=1}^{d}\left(\int_{0}^{t}\left(H_{s}^{j}\right)^{2} d \llbracket \mathbf{X}^{j} \rrbracket_{s}+\int_{0}^{t}\left|H_{s}^{j}\right| \mathrm{V}_{\mathbf{A}^{j}}(d s)\right)<\infty
$$

P-q. s. para todo $t \geq 0$. Para $\mathbf{H} \in \mathcal{L}(\mathbf{X})$, definimos $\mathbf{H} \bullet \mathbf{X} \doteq \sum_{j=1}^{d} \mathbf{H}^{j} \bullet \mathbf{X}^{j}$ e também escrevemos $(\mathbf{H} \bullet \mathbf{X})_{t}=\int_{0}^{t} H_{s} d X_{s}$

Com essas definições as propriedades provadas para o caso escalar valem também para o caso vetorial. 


\subsection{A fórmula de Itô}

Vimos pela fórmula de integração por partes estocástica* que se $\mathbf{X}$ é um semi-martingal contínuo, então $\mathbf{X}^{2}$ também é, pois $\mathbf{X}^{2}$ admite a decomposição

$$
X_{t}^{2}=2 \int_{0}^{t} X_{s} d X_{s}+\llbracket \mathbf{X} \rrbracket_{t}+X_{0}^{2}
$$

Nesta seção apresentaremos a fórmula de Itô, que generaliza este fato. Grosseiramente, esta fórmula diz que $f(\mathbf{X})$ também é um semi-martingal contínuo para qualquer $f$ suficientemente regular. Além deste resultado teórico, a fórmula de Itô também nos permite calcular explicitamente o valor de algumas integráis estocásticas e fazer contas com diferenciais estocásticos. Munido com essas ferramentas, o cálculo estocástico se mostrou útil em diversas aplicações em ciências biológicas e finanças.

Neste capítulo, denotaremos derivadas parciais espaciais de uma função $f \in \mathcal{C}^{2}$ por

$$
D_{i} f=\frac{\partial f}{\partial x_{j}} \text { e } D_{i j}=f \frac{\partial^{2} f}{\partial x_{i} \partial x_{j}}
$$

Seja $G$ um aberto de $\mathbb{R}^{d}$. A notação $f \in \mathcal{C}^{1,2}([0, T] \times G)$ significa que $\frac{\partial f}{\partial t}$ existe em $(0, T) \times G$ e admite uma extensão contínua para $[0, T] \times G$. Além disso, as derivadas parciais com respeito às variáveis espaciais $D_{j} f, D_{i j} f$ existem em $[0, T] \times G$. Isso garante que todas as derivadas parciais são uniformemente contínuas em $[0, T] \times K$ para cada subconjunto compacto $K$ de $G$.

Teorema 4.43 (Fórmula de Itô). Sejam $G \subseteq \mathbb{R}^{d}$ um subconjunto aberto, $\mathbf{X}=\left(\mathbf{X}^{1}, \ldots, \mathbf{X}^{d}\right)$ um semi-martingal contínuo tal que $X_{t}: \Omega \rightarrow G$ e $f \in \mathcal{C}^{2}(G)$. Então para cada $t \geq 0$ vale a fórmula de Itô para quase todo $\omega \in \Omega$

$$
f\left(X_{t}\right)-f\left(X_{0}\right)=\sum_{j=1}^{d} \int_{0}^{t} D_{j} f\left(X_{s}\right) d X_{s}^{j}+\frac{1}{2} \sum_{i, j=1}^{d} \int_{0}^{t} D_{i j} f\left(X_{s}\right) d \llbracket \mathbf{X}^{i}, \mathbf{X}^{j} \rrbracket_{s}
$$

ou, de forma equivalente,

$$
f(\mathbf{X})-f\left(X_{0}\right)=\sum_{j=1}^{d} D_{j} f(\mathbf{X}) \bullet \mathbf{X}^{j}+\frac{1}{2} \sum_{i, j=1}^{d} D_{i j} f(\mathbf{X}) \bullet \llbracket \mathbf{X}^{i}, \mathbf{X}^{j} \rrbracket .
$$

\footnotetext{
* Veja a proposição 4.40 .
} 
Demonstração. Inicialmente, note que pela proposição 4.32, podemos assumir que as trajetórias de $\mathbf{X}$ são contínuas para todo $\omega \in \Omega$. Desta forma, temos que $\mathbf{X} \in \Lambda_{b}$ e portanto as integrais acima existem. Para a demonstração, vamos supor inicialmente que existem compactos $F$ e $K$ tais que $F \subseteq K^{o} \subseteq K \subseteq G$ e a imagem de $\mathbf{X}$ está contida em $F$. Depois relaxaremos essa hipótese. Fixe $t \geq 0$ e considere $\left\{\mathcal{P}_{n}\right\}_{n \geq 1}$ uma sequência de partições do intervalo $[0, t]$ tal que $\left|\mathcal{P}_{n}\right| \rightarrow 0$, quando $n \uparrow \infty$. Escrevemos

$$
\mathcal{P}_{n}: 0=t_{0}^{n}<t_{1}^{n}<\ldots<t_{k_{n}}^{n}=t
$$

Defina $\varepsilon \doteq \operatorname{dist}\left(F, K^{c}\right)>0 \mathrm{e}$

$$
\Omega_{m} \doteq\left\{\omega \in \Omega:\left\|X\left(t_{k}^{n}, \omega\right)-X\left(t_{k-1}^{n}, \omega\right)\right\|<\varepsilon, \forall n \geq m, 1 \leq k \leq k_{n}\right\}
$$

Temos que as trajétorias $s \in[0, t] \mapsto X(s, \omega)$ são uniformemente contínuas para todo $\omega$ e portanto $\omega \in \Omega_{m}$ para algum $m \geq 1$. Isso mostra que $\Omega_{m} \uparrow \Omega$, quando $m \uparrow \infty$. Assim, basta mostrar que a fórmula vale P-q.s. nos conjuntos $\Omega_{m}$, para cada $m \geq 1$. Para isso, fixe $m \geq 1$. Para $\omega \in \Omega_{m}$, $X\left(t_{k}^{n}, \omega\right)$ pertence à esfera $B_{\varepsilon}\left(X\left(t_{k-1}^{n}, \omega\right)\right)$ e portanto o segmento de reta de $X\left(t_{k}^{n}, \omega\right)$ até $X\left(t_{k-1}^{n}, \omega\right)$ está contido em $B_{\varepsilon}\left(X\left(t_{k-1}^{n}, \omega\right)\right) \subseteq K$, para todo $n \geq m$ e $1 \leq k \leq k_{n}$. Considere a expansão de Taylor de segundo grau para $f$ em torno de $X\left(t_{k-1}^{n}, \omega\right)$, isto é,

$$
\begin{aligned}
f\left(X_{t_{k}^{n}}\right)-f\left(X_{t_{k-1}^{n}}\right) & =\sum_{j=1}^{d} D_{j} f\left(X_{t_{k-1}^{n}}\right)\left(X_{t_{k}^{n}}^{j}-X_{t_{k-1}^{n}}^{j}\right) \\
& +\frac{1}{2} \sum_{i, j=1}^{d} D_{i j} f\left(\xi_{n k}\right)\left(X_{t_{k}^{n}}^{i}-X_{t_{k-1}^{n}}^{i}\right)\left(X_{t_{k}^{n}}^{j}-X_{t_{k-1}^{n}}^{j}\right),
\end{aligned}
$$

onde $\xi_{n k}=\xi_{n k}(\omega)$ é um ponto no segmento de reta de $X\left(t_{k}^{n}, \omega\right)$ até $X\left(t_{k-1}^{n}, \omega\right)$. Por outro lado, temos a identidade

$$
f\left(X_{t}\right)-f\left(X_{0}\right)=\sum_{k=1}^{k_{n}}\left[f\left(X_{t_{k}^{n}}\right)-f\left(X_{t_{k-1}^{n}}\right)\right] .
$$

Substituindo (4.27) em (4.26), obtemos

$$
f\left(X_{t}\right)-f\left(X_{0}\right)=\sum_{j=1}^{d} A_{j}^{n}+\frac{1}{2} \sum_{i, j=1}^{d} B_{i j}^{n},
$$


onde

$$
A_{j}^{n}=\sum_{k=1}^{k_{n}} D_{j} f\left(X_{t_{k-1}^{n}}\right)\left(X_{t_{k}^{n}}^{j}-X_{t_{k-1}^{n}}^{j}\right)
$$

$\mathrm{e}$

$$
B_{i j}^{n}=\sum_{k=1}^{k_{n}} D_{i j} f\left(\xi_{n k}\right)\left(X_{t_{k}^{n}}^{i}-X_{t_{k-1}^{n}}^{i}\right)\left(X_{t_{k}^{n}}^{j}-X_{t_{k-1}^{n}}^{j}\right) .
$$

Agora a idéia é a seguinte: Sabemos que

$$
\lim _{n \rightarrow \infty} \sum_{j=1}^{d} A_{j}^{n}+\frac{1}{2} \sum_{i, j=1}^{d} B_{i j}^{n} \stackrel{\mathrm{P}}{\rightarrow} f\left(X_{t}\right)-f\left(X_{0}\right)
$$

em $\Omega$. Vamos provar que

$$
\lim _{n \rightarrow \infty} \sum_{j=1}^{d} A_{j}^{n}+\frac{1}{2} \sum_{i, j=1}^{d} B_{i j}^{n} \stackrel{\mathrm{P}}{\rightarrow} \sum_{j=1}^{d} \int_{0}^{t} D_{j} f\left(X_{s}\right) d X_{s}^{j}+\frac{1}{2} \sum_{i, j=1}^{d} \int_{0}^{t} D_{i j} f\left(X_{s}\right) d \llbracket \mathbf{X}^{\mathbf{i}}, \mathbf{X}^{\mathbf{j}} \rrbracket_{s}
$$

em $\Omega$. pois é conhecido que se $X_{n} \stackrel{\mathrm{P}}{\rightarrow} X$ e $X_{n} \stackrel{\mathrm{P}}{\rightarrow} Y$ então $X=Y$ q. s., logo, para provar o resultado (nas hipóteses simplificadoras explicitadas no começo da demonstração) é suficiente provar (4.28). Para isso, pela proposição B.3 na página 154 basta provarmos a convergência em probabilidade em (4.28) para cada $\Omega_{m}$. Assim, seja $\omega \in \Omega_{m}$. Pela proposição $4.38, A_{j}^{n} \rightarrow \int_{0}^{t} D_{j} f\left(X_{s}\right) d X_{s}^{j}$ em probabilidade quando $n \uparrow \infty$. Resta mostrar que $\lim _{n \rightarrow \infty} B_{i j}^{n} \stackrel{\mathrm{P}}{\rightarrow} \int_{0}^{t} D_{i j} f\left(X_{s}\right) d \llbracket \mathbf{X}^{\mathbf{i}}, \mathbf{X}^{\mathbf{j}} \rrbracket_{s}$ em $\Omega_{m}$. De fato, seja

$$
\tilde{B}_{i j}^{n} \doteq \sum_{k=1}^{k_{n}} D_{i j} f\left(X_{t_{k-1}^{n}}\right)\left(X_{t_{k}^{n}}^{i}-X_{t_{k-1}^{n}}^{i}\right)\left(X_{t_{k}^{n}}^{j}-X_{t_{k-1}^{n}}^{j}\right)
$$

Pelo corolário 4.42, sabemos que $\lim _{n \rightarrow \infty} \tilde{B}_{i j}^{n} \stackrel{\mathrm{P}}{\rightarrow} \int_{0}^{t} D_{i j} f\left(X_{s}\right) d \llbracket \mathbf{X}^{\mathbf{i}}, \mathbf{X}^{\mathbf{j}} \rrbracket_{s}$. Vamos provar que

$$
\lim _{n \rightarrow \infty}\left|B_{i j}^{n}-\tilde{B}_{i j}^{n}\right| \stackrel{\mathrm{P}}{\rightarrow} 0
$$


em $\Omega_{m}$. De fato,

$$
\begin{aligned}
\left|B_{i j}^{n}-\tilde{B}_{i j}^{n}\right| & =\left|\sum_{k=1}^{k_{n}}\left(D_{i j} f\left(\xi_{n k}\right)-D_{i j} f\left(X_{t_{k-1}^{n}}\right)\right)\left(X_{t_{k}^{n}}^{i}-X_{t_{k-1}^{n}}^{i}\right)\left(X_{t_{k}^{n}}^{j}-X_{t_{k-1}^{n}}^{j}\right)\right| \\
& \leq \sup _{1 \leq k \leq k_{n}}\left|D_{i j} f\left(\xi_{n k}\right)-D_{i j} f\left(X_{t_{k-1}^{n}}\right)\right| \sum_{k=1}^{k_{n}}\left|X_{t_{k}^{n}}^{i}-X_{t_{k-1}^{n}}^{i}\right|\left|X_{t_{k}^{n}}^{j}-X_{t_{k-1}^{n}}^{j}\right| \\
& \leq \sup _{1 \leq k \leq k_{n}}\left|D_{i j} f\left(\xi_{n k}\right)-D_{i j} f\left(X_{t_{k-1}^{n}}\right)\right| \sqrt{\sum_{k=1}^{k_{n}}\left(X_{t_{k}^{n}}^{i}-X_{t_{k-1}^{n}}^{i}\right)^{2}} \sqrt{\sum_{k=1}^{k_{n}}\left(X_{t_{k}^{n}}^{j}-X_{t_{k-1}^{n}}^{j}\right)^{2}},
\end{aligned}
$$

onde na última desigualdade usamos a desigualdade de Cauchy-Schwarz. Agora, como $D_{i j} f$ é uniformemente contínua em $K$ temos que

$$
\sup _{1 \leq k \leq k_{n}}\left|D_{i j} f\left(\xi_{n k}\right)-D_{i j} f\left(X_{t_{k-1}^{n}}\right)\right| \longrightarrow 0
$$

P-q. s. e portanto em probabilidade no conjunto $\Omega_{m}$ para $n \uparrow \infty$. Além diso, pela proposição 3.28 na página 57 ,

$$
\sqrt{\sum_{k=1}^{k_{n}}\left(X_{t_{k}^{n}}^{i}-X_{t_{k-1}^{n}}^{i}\right)^{2}} \stackrel{\mathrm{P}}{\rightarrow} \sqrt{\llbracket \mathbf{X}^{i} \rrbracket_{t}}<\infty
$$

e

$$
\sqrt{\sum_{k=1}^{k_{n}}\left(X_{t_{k}^{n}}^{j}-X_{t_{k-1}^{n}}^{j}\right)^{2}} \stackrel{\mathrm{P}}{\rightarrow} \sqrt{\llbracket \mathbf{X}^{j} \rrbracket_{t}}<\infty .
$$

Dessa maneira segue que (4.29).

Agora para o caso geral, tome uma sequência $\left\{K_{m}\right\}_{m \geq 1}$ de compactos tal que $K_{m} \subseteq K_{m+1}^{o}$ e $\cup_{m} K_{m}=G$. Defina $\tau_{m} \doteq \inf \left\{t>0: X_{t} \notin K_{m}\right\}$. Pela contínuidade $\mathbf{X}$ temos que para cada $m \geq 1$, $\tau_{m}$ é tempo de parada e $\tau_{m} \uparrow \infty$ em todo $\Omega$ quando $m \uparrow \infty$. Como $X_{0}$ é constante* podemos escolher $m_{0}$ tal que $X_{0} \in K_{m_{0}}^{o}$ e assim $X_{t}^{\tau_{m}} \in K_{m}$, para $m \geq m_{0}$ e $t \geq 0$. Tome um tal $m$ e usando o resultado provado nas condições anteriores para o processo $\mathbf{X}^{\tau_{m}}$, pela proposição 3.29 na página 58 - item iii)

\footnotetext{
* Veja as observações após a definição 4.34 .
} 
e pelo teorema 4.31 , temos

$$
\begin{aligned}
f(\mathbf{X})^{\tau_{m}}-f\left(X_{0}\right) & =f\left(\mathbf{X}^{\tau_{m}}\right)-f\left(X_{0}\right) \\
& =\sum_{j=1}^{d} D_{j} f\left(\mathbf{X}^{\tau_{m}}\right) \bullet\left(\mathbf{X}^{j}\right)^{\tau_{m}}+\frac{1}{2} \sum_{i, j=1}^{d} D_{i j} f\left(\mathbf{X}^{\tau_{m}}\right) \bullet \llbracket\left(\mathbf{X}^{i}\right)^{\tau_{m}},\left(\mathbf{X}^{j}\right)^{\tau_{m}} \rrbracket \\
& =\sum_{j=1}^{d}\left(D_{j} f(\mathbf{X}) \bullet \mathbf{X}^{j}\right)^{\tau_{m}}+\frac{1}{2} \sum_{i, j=1}^{d}\left(D_{i j} f(\mathbf{X}) \bullet \llbracket \mathbf{X}^{i}, \mathbf{X}^{j} \rrbracket\right)^{\tau_{m}}
\end{aligned}
$$

e finalmente fazendo $m \uparrow \infty$ resulta

$$
f(\mathbf{X})-f\left(X_{0}\right)=\sum_{j=1}^{d} D_{j} f(\mathbf{X}) \bullet \mathbf{X}^{j}+\frac{1}{2} \sum_{i, j=1}^{d} D_{i j} f(\mathbf{X}) \bullet \llbracket \mathbf{X}^{i}, \mathbf{X}^{j} \rrbracket
$$

como queríamos.

Como corolário temos a seguinte versão da fórmula de Itô para funções que dependem do tempo: Corolário 4.44. Sejam $G \subseteq \mathbb{R}^{d}$ um aberto, $T>0, \mathbf{X}=\left(\mathbf{X}^{1}, \ldots, \mathbf{X}^{d}\right)$ um semi-martingal contínuo tal que $X_{t}: \Omega \rightarrow G$ e $f \in \mathcal{C}^{1,2}([0, T] \times G)$. Então

$$
\begin{aligned}
f\left(t, X_{t}\right)-f\left(0, X_{0}\right) & =\int_{0}^{t} \frac{\partial f}{\partial s}\left(s, X_{s}\right) d s+\sum_{j=1}^{d} \int_{0}^{t} D_{j} f\left(s, X_{s}\right) d X_{s}^{j} \\
& +\frac{1}{2} \sum_{i, j=1}^{d} \int_{0}^{t} D_{i j} f\left(s, X_{s}\right) d \llbracket \mathbf{X}^{i}, \mathbf{X}^{j} \rrbracket_{s},
\end{aligned}
$$

P-q.s., para cada $t \in[0, T]$.

Demonstração. Seja $\mathcal{P}_{n}: 0=t_{0}^{n}<t_{1}^{n}<\ldots<t_{k_{n}}^{n}=t$ uma partição do intervalo $[0, t]$. Temos que

$$
\begin{aligned}
f\left(t, X_{t}\right)-f\left(0, X_{0}\right) & =\sum_{k=1}^{k_{n}}\left[f\left(t_{k}^{n}, X_{t_{k}^{n}}\right)-f\left(t_{k-1}^{n}, X_{t_{k-1}^{n}}\right)\right] \\
& =\sum_{k=1}^{k_{n}}\left[\left(f\left(t_{k}^{n}, X_{t_{k}^{n}}\right)-f\left(t_{k-1}^{n}, X_{t_{k}^{n}}\right)\right)+\left(f\left(t_{k-1}^{n}, X_{t_{k}^{n}}\right)-f\left(t_{k-1}^{n}, X_{t_{k-1}^{n}}\right)\right)\right] .
\end{aligned}
$$


Porém, pelo teorema do valor médio,

$$
f\left(t_{k}^{n}, X_{t_{k}^{n}}\right)-f\left(t_{k-1}^{n}, X_{t_{k}^{n}}\right)=\frac{\partial f}{\partial t}\left(\eta_{n k}, X_{t_{k}^{n}}\right)\left(t_{k}^{n}-t_{k-1}^{n}\right)
$$

para algum $\eta_{n k}=\eta_{n k}(\omega) \in\left(t_{k-1}^{n}, t_{k}^{n}\right)$. Ficamos portanto com

$$
f\left(t, X_{t}\right)-f\left(0, X_{0}\right)=\sum_{k=1}^{k_{n}} \frac{\partial f}{\partial t}\left(\eta_{n k}, X_{t_{k}^{n}}\right)\left(t_{k}^{n}-t_{k-1}^{n}\right)+\sum_{k=1}^{k_{n}}\left(f\left(t_{k-1}^{n}, X_{t_{k}^{n}}\right)-f\left(t_{k-1}^{n}, X_{t_{k-1}^{n}}\right)\right)
$$

e tratamos a segunda somatória da mesma forma como na demonstração do teorema anterior.

Conforme mencionamos no parágrafo inicial desta seção, a fórmula de Itô mostra, em particular, que a classe dos semi-martingais contínuos é fechada pela aplicação de funções em $\mathcal{C}^{2}$. De maneira mais precisa temos o

Corolário 4.45. Sejam $G \subseteq \mathbb{R}^{d}$ um subconjunto aberto, $f \in \mathcal{C}^{2}(G)$ e $\mathbf{X}: \Omega \rightarrow G$ um semi-martingal contínuo d-dimensional, tal que $\mathbf{X}^{i}=\mathbf{M}^{i}+\mathbf{A}^{i}$ seja a decomposição semi-martingal para $\mathbf{X}^{i}$ para cada $i=1, \ldots, d$. Então $f(\mathbf{X})$ também é um semi-martingal contínuo.

Demonstração. Vamos provar a afirmação exibindo a decomposição semi-martingal de $f(\mathbf{X})$. De fato, pela fórmula de Itô, temos

$$
\begin{aligned}
f(\mathbf{X}) & =f\left(X_{0}\right)+\sum_{i=1}^{d} D_{i} f(\mathbf{X}) \bullet \mathbf{X}^{i}+\frac{1}{2} \sum_{i, j=1}^{d} D_{i j} f(\mathbf{X}) \bullet \llbracket \mathbf{X}^{i}, \mathbf{X}^{j} \rrbracket \\
& =f\left(X_{0}\right)+\sum_{i=1}^{d} D_{i} f(\mathbf{X}) \bullet \mathbf{M}^{i}+ \\
& +\left[\sum_{i=1}^{d} D_{i} f(\mathbf{X}) \bullet \mathbf{A}^{i}+\frac{1}{2} \sum_{i, j=1}^{d} D_{i j} f(\mathbf{X}) \bullet \llbracket \mathbf{X}^{i}, \mathbf{X}^{j} \rrbracket\right] \\
& =\mathbf{M}+\mathbf{A},
\end{aligned}
$$

onde

$$
\mathbf{M}=f\left(X_{0}\right)+\sum_{i=1}^{d} D_{i} f(\mathbf{X}) \bullet \mathbf{M}^{i}
$$


$\mathrm{e}$

$$
\mathbf{A}=\sum_{i=1}^{d} D_{i} f(\mathbf{X}) \bullet \mathbf{A}^{i}+\frac{1}{2} \sum_{i, j=1}^{d} D_{i j} f(\mathbf{X}) \bullet \llbracket \mathbf{X}^{i}, \mathbf{X}^{j} \rrbracket .
$$

O resultado segue pois para cada $i=1, \ldots, d, \mathbf{M}^{i}$ é martingal local contínuo e $\mathbf{A}^{i}$ um processo contínuo de variação finita que se anula em $t=0$.

Exemplo 4.46. Vamos mostrar como a fórmula de Itô pode ser útil para o cálculo de integrais estocásticas. Seja $\mathbf{B}$ um movimento Browniano padrão. Lembramos que $\llbracket \mathbf{B} \rrbracket_{t}=t$ para todo $t \geq 0$ * Assim, nas condições da fórmula de Itô, temos que

$$
\begin{aligned}
f\left(t, B_{t}\right)-f\left(0, B_{0}\right) & =\int_{0}^{t} \frac{\partial f}{\partial t}\left(s, B_{s}\right) d s+\int_{0}^{t} \frac{\partial f}{\partial x}\left(s, B_{s}\right) d B_{s} \\
& +\frac{1}{2} \int_{0}^{t} \frac{\partial^{2} f}{\partial x^{2}}\left(s, B_{s}\right) d \llbracket \mathbf{B} \rrbracket_{s} \\
& =\int_{0}^{t} \frac{\partial f}{\partial t}\left(s, B_{s}\right) d s+\int_{0}^{t} \frac{\partial f}{\partial x}\left(s, B_{s}\right) d B_{s} \\
& +\frac{1}{2} \int_{0}^{t} \frac{\partial^{2} f}{\partial x^{2}}\left(s, B_{s}\right) d s
\end{aligned}
$$

e portanto

$$
\int_{0}^{t} \frac{\partial f}{\partial x}\left(s, B_{s}\right) d B_{s}=\left.f\left(s, B_{s}\right)\right|_{0} ^{t}-\int_{0}^{t} \frac{\partial f}{\partial t}\left(s, B_{s}\right)+\frac{1}{2} \frac{\partial^{2} f}{\partial x^{2}}\left(s, B_{s}\right) d s .
$$

Em particular, quando $f$ não depende de $t$, ficamos com

$$
\int_{0}^{t} \frac{\partial f}{\partial x}\left(B_{s}\right) d B_{s}=\left.f\left(B_{s}\right)\right|_{0} ^{t}-\int_{0}^{t} \frac{1}{2} \frac{\partial^{2} f}{\partial x^{2}}\left(B_{s}\right) d s
$$

Como exemplo concreto, vamos calcular $\int_{0}^{t} B_{s} e^{B_{s}} d B_{s}$. Nesse caso, temos que $f^{\prime}(x)=x e^{x}$ e portanto

\footnotetext{
* Veja o exemplo 3.14 na página 49.
} 
$f(x)=x e^{x}-e^{x}+C$ e $f^{\prime \prime}(x)=e^{x} x+e^{x}$. Assim, ficamos com

$$
\begin{aligned}
\int_{0}^{t} B_{s} e^{B_{s}} d B_{s} & =\left.\left(B_{s} e^{B_{s}}-e^{B_{s}}\right)\right|_{0} ^{t}-\int_{0}^{t} \frac{1}{2}\left(e^{B_{s}} B_{s}+e^{B_{s}}\right) d s \\
& =\left(B_{t}-1\right) e^{B_{t}}+1-\frac{1}{2} \int_{0}^{t} e^{B_{s}}\left(B_{s}+1\right) d s .
\end{aligned}
$$

\subsubsection{Notação diferencial}

Definição 4.47. Seja $\mathbf{X}$ um semi-martingal contínuo. Escrevemos $d Z_{t}=H_{t} d X_{t}$ ou $d \mathbf{Z}=\mathbf{H} d \mathbf{X}$ quando $\mathbf{H} \in \mathcal{L}(\mathbf{X})$ e $Z_{t}=Z_{0}+\int_{0}^{t} H_{s} d X_{s}$ para todo $t \geq 0$, ou equivalentemente, quando $\mathbf{H} \in \mathcal{L}(\mathbf{X}) e$ $\mathbf{Z}=Z_{0}+\mathbf{H} \bullet \mathbf{X}$.

Observamos que a expressão $d \mathbf{Z}=0$ deve ser interpreada como $d \mathbf{Z}=0 d \mathbf{X}$, para algum semimartingal contínuo $\mathbf{X}$. Nesse caso temos que $Z_{t}=Z_{0}$ para todo $t \geq 0$, isto é, $\mathbf{Z}$ é um processo constante. Veremos agora consequências da propriedade associativa da integral estocástica na manipulação das diferenciais estocásticas.

Proposição 4.48. Sejam $\mathbf{X}$ e $\mathbf{Y}$ semi-martingais contínuos. Se $d \mathbf{Z}=\mathbf{H} d \mathbf{X}$ e dX $\mathbf{X}=\mathbf{K} d \mathbf{Y}$, então $d \mathbf{Z}=\mathbf{H K} d \mathbf{Y}$.

Demonstração. De fato, temos que $\mathbf{H} \in \mathcal{L}(\mathbf{X}), \mathbf{K} \in \mathcal{L}(\mathbf{Y}), \mathbf{Z}=Z_{0}+\mathbf{H} \bullet \mathbf{X}$ e $\mathbf{X}=X_{0}+\mathbf{K} \bullet \mathbf{Y}$. Logo,

$$
\begin{aligned}
\mathbf{Z} & =Z_{0}+\mathbf{H} \bullet\left(X_{0}+\mathbf{K} \bullet \mathbf{Y}\right) \\
& =Z_{0}+\mathbf{H} \bullet(\mathbf{K} \bullet \mathbf{Y}) \\
& =Z_{0}+(\mathbf{H K}) \bullet \mathbf{Y}
\end{aligned}
$$

onde na última igualdade usamos o teorema 4.33. Segue portanto da definição anterior que $d \mathbf{Z}=\mathbf{H K} d \mathbf{Y}$.

Proposição 4.49. Sejam $\mathbf{X}, \mathbf{Y}$ semi-martingais contínuos, $\mathbf{H} \in \mathcal{L}(\mathbf{X})$ e $\mathbf{K} \in \mathcal{L}(\mathbf{Y})$. Defina também $\mathbf{Z} \doteq \mathbf{H} \bullet \mathbf{X} e \mathbf{W} \doteq \mathbf{K} \bullet \mathbf{Y}$. Então $d \llbracket \mathbf{Z}, \mathbf{W} \rrbracket=\mathbf{H K} d \llbracket \mathbf{X}, \mathbf{Y} \rrbracket$. 
Demonstração. De fato, pelo teorema 4.31 e como $\llbracket \mathbf{H} \bullet \mathbf{X}, \mathbf{K} \bullet \mathbf{Y} \rrbracket_{0}=0$, que

$$
\begin{aligned}
\llbracket \mathbf{H} \bullet \mathbf{X}, \mathbf{K} \bullet \mathbf{Y} \rrbracket & =\mathbf{H K} \bullet \llbracket \mathbf{X}, \mathbf{Y} \rrbracket \\
& =\llbracket \mathbf{H} \bullet \mathbf{X}, \mathbf{K} \bullet \mathbf{Y} \rrbracket_{0}+\mathbf{H K} \bullet \llbracket \mathbf{X}, \mathbf{Y} \rrbracket
\end{aligned}
$$

e portanto, pela definição 4.47 , segue que $d \llbracket \mathbf{Z}, \mathbf{W} \rrbracket=\mathbf{H K} d \llbracket \mathbf{X}, \mathbf{Y} \rrbracket$.

Esse último resultado nos induz a definir o produto de diferenciais estocástica como

$$
d \mathbf{Z} d \mathbf{W} \doteq d \llbracket \mathbf{Z}, \mathbf{W} \rrbracket
$$

pois dessa maneira (e nas hipóteses da proposição anterior)

$$
d \mathbf{Z} d \mathbf{W}=d \llbracket \mathbf{Z}, \mathbf{W} \rrbracket=\mathbf{H K} \llbracket \mathbf{X}, \mathbf{Y} \rrbracket=\mathbf{H K} d \mathbf{X} d \mathbf{Y}
$$

e em particular $d \llbracket \mathbf{Z} \rrbracket=(d \mathbf{Z})^{2}=\mathbf{H}^{2}(d \mathbf{X})^{2}=H^{2} d \llbracket \mathbf{X} \rrbracket$. Além disso a fórmula de Itô em notação diferencial fica

$$
d f\left(X_{t}\right)=\sum_{j=1}^{d} D_{j} f\left(X_{t}\right) d X_{t}^{j}+\frac{1}{2} \sum_{i, j=1}^{d} D_{i j} f\left(X_{t}\right) d \llbracket \mathbf{X}^{i}, \mathbf{X}^{j} \rrbracket_{t}
$$

$\mathrm{e}$

$$
d f\left(t, X_{t}\right)=\frac{\partial f}{\partial t}\left(t, X_{t}\right) d t+\sum_{j=1}^{d} D_{j} f\left(t, X_{t}\right) d X_{t}^{j}+\frac{1}{2} \sum_{i, j=1}^{d} D_{i j} f\left(t, X_{t}\right) d \llbracket \mathbf{X}^{i}, \mathbf{X}^{j} \rrbracket_{t} .
$$

Para o caso particular de um semi-martingal escalar $(d=1)$ temos

$$
d f\left(X_{t}\right)=f^{\prime}\left(X_{t}\right) d X_{t}+\frac{1}{2} f^{\prime \prime}\left(X_{t}\right) d \llbracket \mathbf{X} \rrbracket_{t}
$$

$\mathrm{e}$

$$
d f\left(t, X_{t}\right)=\frac{\partial f}{\partial t}\left(t, X_{t}\right) d t+\frac{\partial f}{\partial x}\left(t, X_{t}\right) d X_{t}+\frac{1}{2} \frac{\partial^{2} f}{\partial x^{2}}\left(t, X_{t}\right) d \llbracket \mathbf{X} \rrbracket_{t} .
$$

Observe que aplicando a fórmula de Itô com $f(x)=x^{2}$, temos que $d X_{t}^{2}=2 X_{t} d X_{t}+d \llbracket \mathbf{X} \rrbracket_{t}=$ $2 X_{t} d X_{t}+\left(d X_{t}\right)^{2}$. No cálculo clássico, as funções são de variação limitada e pela regra da cadeia, valeria que $d X_{t}^{2}=2 X_{t} d X_{t}$. Note que o último termo não aparece, afinal, para funções de variação limitada a variação quadrática é nula! Assim, pode-se dizer que a fórmula de Itô é uma generalização da regra da cadeia. Terminaremos esta seção com mais uma aplicação da fórmula de Itô usada com 
a notação diferencial.

Proposição 4.50. Sejam $G_{1}, G_{2} \subseteq \mathbb{R}$ conjuntos abertos, $\mathbf{X}: \Omega \rightarrow G_{1}, \mathbf{Y}: \Omega \rightarrow G_{2}$ semi-martingais contínuos, $f \in \mathcal{C}^{2}\left(G_{1}\right)$ e $g \in \mathcal{C}^{2}\left(G_{2}\right)$. Então

i) $\llbracket f(\mathbf{X}), g(\mathbf{Y}) \rrbracket=\int_{0}^{t} f^{\prime}\left(X_{s}\right) g^{\prime}\left(X_{s}\right) d \llbracket \mathbf{X}, \mathbf{Y} \rrbracket_{s}$, ou seja, $d \llbracket f(\mathbf{X}), g(\mathbf{Y}) \rrbracket_{t}=f^{\prime}\left(X_{t}\right) g^{\prime}\left(Y_{t}\right) d \llbracket \mathbf{X}, \mathbf{Y} \rrbracket_{t}$.

ii) Se $\mathbf{M}$ for um semi-martingal contínuo e positivo então $\llbracket \mathbf{X}, \log (\mathbf{M}) \rrbracket_{t}=\int_{0}^{t} M_{s}^{-1} d \llbracket \mathbf{X}, \mathbf{M} \rrbracket_{s}$, ou seja, $d \llbracket \mathbf{X}, \log (\mathbf{M}) \rrbracket_{t}=M_{t}^{-1} d \llbracket \mathbf{X}, \mathbf{M} \rrbracket_{t}$.

iii) Se $\mathbf{X}$ for um semi-martingal contínuo e positivo então $\int_{0}^{t} X_{s}^{-1} d X_{s}=\log \left(X_{t}\right)-\log \left(X_{0}\right)+$ $\frac{1}{2} \llbracket \log (\mathbf{X}) \rrbracket_{t}$.

iv) $\mathbf{X Y}$ é semi-martingal contínuo $e^{*} u_{\mathbf{X Y}}(t)=\int_{0}^{t}\left(X_{s} d_{u_{\mathbf{Y}}}(s)+Y_{s} d_{u_{\mathbf{X}}}(s)\right)+\llbracket \mathbf{X}, \mathbf{Y} \rrbracket_{t}$, ou seja, $d_{u_{\mathbf{X Y}}}(t)=X_{t} d_{u_{\mathbf{Y}}}(t)+Y_{t} d_{u_{\mathbf{X}}}(t)+d \llbracket \mathbf{X}, \mathbf{Y} \rrbracket_{t}$.

Demonstração. i) Pela fórmula de Itô temos

$$
f\left(X_{t}\right)=f\left(X_{0}\right)+\int_{0}^{t} f^{\prime}\left(X_{s}\right) d X_{s}+\frac{1}{2} \int_{0}^{t} f^{\prime \prime}\left(X_{s}\right) d \llbracket \mathbf{X} \rrbracket_{s}=\left(f^{\prime}(\mathbf{X}) \bullet \mathbf{X}\right)_{t}+A_{t}
$$

$\mathrm{e}$

$$
g\left(X_{t}\right)=g\left(X_{0}\right)+\int_{0}^{t} g^{\prime}\left(X_{s}\right) d X_{s}+\frac{1}{2} \int_{0}^{t} g^{\prime \prime}\left(X_{s}\right) d \llbracket \mathbf{X} \rrbracket_{s}=\left(g^{\prime}(\mathbf{X}) \bullet \mathbf{X}\right)_{t}+B_{t},
$$

com A e B processos de variação finita. Pela definição de co-variação quadrática para semimartingais ${ }^{\dagger}$ temos que podemos desconsiderar os processos de variação finita e assim pelo teorema 4.31 , resulta

$$
\llbracket f(\mathbf{X}), g(\mathbf{Y}) \rrbracket=\llbracket f^{\prime}(\mathbf{X}) \bullet \mathbf{X}, g^{\prime}(\mathbf{Y}) \bullet \mathbf{Y} \rrbracket_{t}=\int_{0}^{t} f^{\prime}\left(X_{s}\right) g^{\prime}\left(X_{s}\right) d \llbracket \mathbf{X}, \mathbf{Y} \rrbracket_{s} .
$$

ii) Resulta por i) tomando $f(x)=x, \mathbf{Y}=\mathbf{M}$ e $g(y)=\log (y)$.

\footnotetext{
* Lembre-se que $u_{\mathbf{X}}$ é o compensador do semi-martingal X. Veja a definição 3.25 na página 56 . † Veja a definição 3.27 na página 57.
} 
iii) Note inicialmente que $\llbracket \log (\mathbf{X}) \rrbracket_{0}=0$. Assim, podemos escrever o que queremos provar como

$$
\log \left(X_{t}\right)+\frac{1}{2} \llbracket \log (\mathbf{X}) \rrbracket_{t}=\log \left(X_{0}\right)+\frac{1}{2} \llbracket \log (\mathbf{X}) \rrbracket_{0}+\int_{0}^{t} X_{s}^{-1} d X_{s}
$$

Agora, de acordo com a notação diferencial, isto é equivalente a mostrar que

$$
d\left(\log \left(X_{t}\right)+\frac{1}{2} \llbracket \log (\mathbf{X}) \rrbracket_{t}\right)=d \log \left(X_{t}\right)+\frac{1}{2} d \llbracket \log (\mathbf{X}) \rrbracket_{t}=X_{t}^{-1} d X_{t}
$$

Vamos mostrar que (4.31) vale. De fato, pela fórmula de Itô temos que

$$
d \log \left(X_{t}\right)=X_{t}^{-1} d X_{t}-\frac{1}{2} X_{t}^{-2} d \llbracket \mathbf{X} \rrbracket_{t}
$$

e por i), usando $\mathbf{X}=\mathbf{Y}$ e $f(x)=g(x)=\log (x)$, ficamos com

$$
\begin{aligned}
\llbracket \log (\mathbf{X}) \rrbracket_{t}=\llbracket \log (\mathbf{X}), \log (\mathbf{X}) \rrbracket_{t} & =\int_{0}^{t} X_{s}^{-2} d \llbracket \mathbf{X} \rrbracket_{s} \\
& =\llbracket \log (\mathbf{X}) \rrbracket_{0}+\left(X^{2} \bullet \llbracket \mathbf{X} \rrbracket\right)_{t}
\end{aligned}
$$

e portanto, pela definição da notação diferencial

$$
d \llbracket \log (\mathbf{X}) \rrbracket_{t}=X_{t}^{-2} d \llbracket \mathbf{X} \rrbracket_{t}
$$

Substituindo (4.33) em (4.32), obtemos (4.31).

iv) Sejam $\mathbf{X}=\mathbf{M}+\mathbf{A}, \mathbf{Y}=\mathbf{N}+\mathbf{C}$ as decomposições semi-martingais de $\mathbf{X}$ e $\mathbf{Y}\left(u_{\mathbf{X}}=\mathbf{A}, u_{\mathbf{Y}}=\right.$ C). Pela proposição 4.40, temos que

$$
\begin{aligned}
X_{t} Y_{t} & =X_{0} Y_{0}+\int_{0}^{t} X_{s} d Y_{s}+\int_{0}^{t} Y_{s} d X_{s}+\llbracket \mathbf{X}, \mathbf{Y} \rrbracket_{t} \\
& =\left(X_{0} Y_{0}+\int_{0}^{t} X_{s} d N_{s}+\int_{0}^{t} Y_{s} d M_{s}\right)+\left(\int_{0}^{t} X_{s} d C_{s}+\int_{0}^{t} Y_{s} d A_{s}+\llbracket \mathbf{X}, \mathbf{Y} \rrbracket_{t}\right) .
\end{aligned}
$$

Note que o primeiro termo da soma é um martingal local contínuo e o segundo é processo contínuo, de 
variação finita e que se anula em $t=0$. Isso mostra que $\mathbf{X Y}$ é um semi-martingal com compensador $u_{\mathbf{X Y}}(t)=\int_{0}^{t} X_{s} d C_{s}+\int_{0}^{t} Y_{s} d A_{s}+\llbracket \mathbf{X}, \mathbf{Y} \rrbracket_{t}$. Como queríamos.

\subsubsection{Caracterização de Lévy e a tabela de cálculo para o movimento Browniano}

Apresentaremos aqui uma caracterização para o movimento Browniano. Este resultado foi obtido originalmente por P. Lévy e a prova que apresentaremos aqui, utilizando o lema de Itô é de autoria de Kunita e Watanabe. Lembramos que um movimento Browniano $d$-dimensional começando em $0 \in \mathbb{R}^{d}$ é um processo estocástico em $\mathbb{R}^{d}, B_{t}=\left(B_{t}^{1}, B_{t}^{2}, \ldots, B_{t}^{d}\right)$ tal que os processos unidimensionais $\mathbf{B}^{1}, \ldots, \mathbf{B}^{d}$ são mutualmente independentes e para cada $j \in\{1,2, \ldots, d\}, \mathbf{B}^{j}$ é um movimento browniano padrão unidimensional. Agora, para começar, precisaremos de um lema.

Lema 4.51. Seja $B_{t}=\left(B_{t}^{1}, B_{t}^{2}, \ldots, B_{t}^{d}\right)$ um movimento Browniano definido em $\left(\Omega, \mathscr{F},\left\{\mathscr{F}_{t}\right\}, \mathrm{P}\right)$. Então para $i \neq j, \mathbf{B}^{i} \mathbf{B}^{j}$ é um martingal.

Demonstração. Sabemos que para cada $j=1,2, \ldots, d, \mathbf{B}^{j}$ é um martingal de quadrado integrável*. Dessa forma, $B_{t}^{j} \in \mathrm{L}^{2}$ e portanto, pela desigualdade de Hölder, $B_{t}^{i} B_{t}^{j} \in \mathrm{L}^{1}$ para todo $i, j$ e $t \geq$ 0 . Agora, suponha que $i \neq j$ e $0 \leq s \leq t$. Devemos mostrar que $\mathbf{E}\left(B_{t}^{i} B_{t}^{j} \| \mathscr{F}_{s}\right)=B_{s}^{i} B_{s}^{j}$ ou, equivalentemente, $\mathbf{E}\left(B_{t}^{i} B_{t}^{j}-B_{s}^{i} B_{s}^{j} \| \mathscr{F}_{s}\right)=0$. Para isso, note incialmente que

$$
B_{t}^{i} B_{t}^{j}-B_{s}^{i} B_{s}^{j}=\left(B_{t}^{i}-B_{s}^{i}\right)\left(B_{t}^{j}-B_{s}^{j}\right)+B_{s}^{i}\left(B_{t}^{j}-B_{s}^{j}\right)+B_{s}^{j}\left(B_{t}^{i}-B_{s}^{i}\right) .
$$

Note também que $\left(B_{t}^{i}-B_{s}^{i}\right)\left(B_{t}^{j}-B_{s}^{j}\right)$ é independente da $\sigma$-álgebra $\mathscr{F}_{s}$ pois é uma função do incremento $B_{t}-B_{s}$. Além disso, os fatores $\left(B_{t}^{i}-B_{s}^{i}\right)$ e $\left(B_{t}^{j}-B_{s}^{j}\right)$ são independentes entre si. Assim, pelo teorema C.12 na página 161, temos que

$$
\begin{aligned}
\mathbf{E}\left(\left(B_{t}^{i}-B_{s}^{i}\right)\left(B_{t}^{j}-B_{s}^{j}\right) \| \mathscr{F}_{s}\right) & =\mathbf{E}\left(\left(B_{t}^{i}-B_{s}^{i}\right)\left(B_{t}^{j}-B_{s}^{j}\right)\right) \\
& =\mathbf{E}\left(\left(B_{t}^{i}-B_{s}^{i}\right)\right) \mathbf{E}\left(\left(B_{t}^{j}-B_{s}^{j}\right)\right) \\
& =0 .
\end{aligned}
$$

Agora, como $B_{s}^{i}$ e $B_{s}^{j}$ são $\mathscr{F}_{s}$-mensuráveis, temos que

$$
\mathbf{E}\left(B_{s}^{i}\left(B_{t}^{j}-B_{s}^{j}\right)|| \mathscr{F}_{s}\right)=B_{s}^{i} \mathbf{E}\left(\left(B_{t}^{j}-B_{s}^{j}\right)|| \mathscr{F}_{s}\right)=0,
$$

\footnotetext{
* Veja o exemplo 3.2 na página 34.
} 
e

$$
\mathbf{E}\left(B_{s}^{j}\left(B_{t}^{i}-B_{s}^{i}\right) \| \mathscr{F}_{s}\right)=B_{s}^{j} \mathbf{E}\left(\left(B_{t}^{i}-B_{s}^{i}\right) \| \mathscr{F}_{s}\right)=0 .
$$

Desses fatos resulta que $\mathbf{E}\left(B_{t}^{i} B_{t}^{j}-B_{s}^{i} B_{s}^{j} \| \mathscr{F}_{s}\right)=0$, como queríamos.

Teorema 4.52 (Caracterização de Lévy). Seja $\mathbf{B}=\left(\mathbf{B}^{1}, \mathbf{B}^{2}, \ldots, \mathbf{B}^{d}\right)$ um martingal local contínuo ddimensional. Então $\mathbf{B}$ é um movimento Browniano em $\left(\Omega, \mathscr{F},\left\{\mathscr{F}_{t}\right\}, \mathrm{P}\right)$ se, e somente se, $\llbracket \mathbf{B}^{i} \rrbracket_{t}=t$ $e \llbracket \mathbf{B}^{i}, \mathbf{B}^{j} \rrbracket_{t}=0$, para todo $t \geq 0$ e $i, j \in\{1, \ldots, d\}$ com $i \neq j$.

Demonstração. Conforme observamos na demonstração do lema anterior, cada processo $\mathbf{B}^{i}$ é um martingal contínuo de quadrado integrável e portanto um martingal local contínuo. Pela proposição 3.16 na página 50, temos que a covariação $\llbracket \mathbf{B}^{i}, \mathbf{B}^{j} \rrbracket$ é o único processo contínuo de variação finita, nulo na origem e tal que $\mathbf{B}^{i} \mathbf{B}^{j}-\llbracket \mathbf{B}^{i}, \mathbf{B}^{j} \rrbracket$ é um martingal local. Mas pelo lema anterior, quando $i \neq j$, $\mathbf{B}^{i} \mathbf{B}^{j}$ é um martingal (e portanto um martingal local) isso nos força a concluir que $\llbracket \mathbf{B}^{i}, \mathbf{B}^{j} \rrbracket_{t}=0$ para todo $t \geq 0$ e quando $i=j$, vimos pelo exemplo 3.14 na página 49 que $\llbracket \mathbf{B}^{i} \rrbracket_{t}=t$.

Reciprocamente, como B é contínuo por hipótese, para provar que é um movimento Browniano devemos mostrar que para cada $0 \leq s<t$ o incremento $\mathbf{B}_{t}-\mathbf{B}_{s}$ é independente da $\sigma$-álgebra $\mathscr{F}_{s}$ e é normal multivariado com vetor de média nulo e matriz de covariância $(t-s) I$. Para isto, fixe $\alpha \in \mathbb{R}^{d}$ e defina $\phi_{\alpha}: \mathbb{R} \times \mathbb{R}^{d} \rightarrow \mathbb{C} \in \mathcal{C}^{2}\left(\mathbb{R}^{d+1}\right)$ como

$$
\phi_{\alpha}(t, x)=e^{\imath \alpha \cdot x+\frac{1}{2}\|\alpha\| t}
$$

Calculando as derivadas parciais temos $D_{j j} \phi_{\alpha}=-\alpha_{j}^{2} \phi_{\alpha}$ e $\frac{\partial \phi_{\alpha}}{\partial t}=\frac{1}{2}\|\alpha\|^{2} \phi_{\alpha}$. Portanto

$$
\frac{\partial \phi_{\alpha}}{\partial t}+\frac{1}{2} \sum_{j=1}^{d} D_{j j} \phi_{\alpha}=0
$$

Defina $X_{t}=\phi_{\alpha}\left(t, B_{t}\right)$. Usando as hipóteses e relação acima, pela fórmula de Itô vem que

$$
d X_{t}=\sum_{j=1}^{d} D_{j} \phi_{\alpha}\left(t, B_{t}\right) d B_{t}^{j}
$$


pela definição da notação diferencial, isso significa que

$$
\mathbf{X}=X_{0}+\sum_{j=1}^{d} D_{j} \phi_{\alpha}\left(t, B_{t}\right) \bullet \mathbf{B}^{j}
$$

Ainda pela fórmula de Itô* ${ }^{*}$ resulta que $\mathbf{X}$ é um semi-martingal contínuo e pela relação acima vemos que $\mathbf{X}$ é na verdade um martingal local contínuo. Vamos mostrar que na verdade $\mathbf{X}$ é um martingal de quadrado integrável. De fato, seja $a>0$ arbitrário. Temos que para $t \in[0, a],\left|\phi_{\alpha}(t, x)\right| \leq e^{\frac{1}{2}\|\alpha\|^{2} a}$ e portanto $\left|X_{t}\right| \leq e^{\frac{1}{2}\|\alpha\|^{2} a}$. Isso mostra que $\left\{X_{t}\right\}_{t \in[0, a]}$ é um martingal uniformemente limitado, e portanto, de quadrado integrável. Da arbitrariedade de $a>0$ segue que $\mathbf{X}$ é um martingal de quadrado integrável. Vamos usar esse resultado para provar o resto do teorema. Começaremos provando que a matriz de covariância de $B_{t}-B_{s}$ é $(t-s) I$ para isso, sabemos que basta mostrar que a função característica de $B_{t}-B_{s}$, isto é, $\mathbf{E}\left(e^{\imath \alpha \cdot\left(B_{t}-B_{s}\right)}\right)$ vale $e^{-\frac{1}{2}\|\alpha\|^{2}(t-s)}$. De fato, como $\mathbf{X}$ é um martingal, temos que

$$
\mathbf{E}\left(e^{\imath \alpha \cdot B_{t}+\frac{1}{2}\|\alpha\|^{2} t} \| \mathscr{F}_{s}\right)=e^{\imath \alpha \cdot B_{s}+\frac{1}{2}\|\alpha\|^{2} s} .
$$

Multiplicando dos dois lados da igualdade por $e^{-\imath \alpha \cdot B_{s}-\frac{1}{2}\|\alpha\|^{2} t}$. Como este fator é limitado e $\mathscr{F}_{s^{-}}$ mensurável, pelo teorema C.10 na página 160 ficamos com

$$
\mathbf{E}\left(e^{\imath \alpha \cdot\left(B_{t}-B_{s}\right)} \| \mathscr{F}_{s}\right)=e^{-\frac{1}{2}\|\alpha\|^{2}(t-s)} .
$$

Integrando sobre $\Omega$ e usando a definição de esperança condicional ${ }^{\dagger}$ ficamos com

$$
\mathbf{E}\left(e^{\imath \alpha \cdot\left(B_{t}-B_{s}\right)}\right)=e^{-\frac{1}{2}\|\alpha\|^{2}(t-s)} .
$$

Finalmente, para provar que $B_{t}-B_{s}$ é independente da $\sigma$-álgebra $\mathscr{F}_{s}$, basta provar que $B_{t}-B_{s}$ é independente de toda variável aleatória $\xi \mathscr{F}_{s}$-mensurável. Para isso, devemos mostrar que a função característica do vetor aleatório $\left(B_{t}-B_{s}, \xi\right)$ se fatora no produto das funções características de $B_{t}-B_{s}$

\footnotetext{
* Veja o corolário 4.45. † Mais especificamente, veja o item ii) da definição C.6 na página 158.
} 
e $\xi$, isto é, devemos mostrar que $\mathbf{E}\left(e^{\imath\left(B_{t}-B_{s}, \xi\right) \cdot(\alpha, \beta)}\right)=e^{-\frac{1}{2}\|\alpha\|^{2}(t-s)} \mathbf{E}\left(e^{\imath \beta \xi}\right)$. Para isso, observe

$$
\begin{aligned}
\mathbf{E}\left(e^{\imath\left(B_{t}-B_{s}, \xi\right) \cdot(\alpha, \beta)}\right) & =\mathbf{E}\left(e^{\imath \beta \xi+\imath \alpha \cdot\left(B_{t}-B_{s}\right)}\right) \\
& =\mathbf{E}\left(\mathbf{E}\left(e^{\imath \beta \xi} e^{\imath \alpha \cdot\left(B_{t}-B_{s}\right)} \| \mathscr{F}_{s}\right)\right) \\
& =\mathbf{E}\left(e^{\imath \beta \xi} \mathbf{E}\left(e^{\imath \alpha \cdot\left(B_{t}-B_{s}\right)} \| \mathscr{F}_{s}\right)\right) \\
& =\mathbf{E}\left(e^{\imath \beta \xi} e^{-\frac{1}{2}\|\alpha\|^{2}(t-s)}\right) \\
& =e^{-\frac{1}{2}\|\alpha\|^{2}(t-s)} \mathbf{E}\left(e^{\imath \beta \xi}\right) .
\end{aligned}
$$

Na quarta igualdade usamos (4.34). Com isso terminamos a demonstração do teorema.

Agora, provaremos um resultado muito usado nas aplicações de cálculo estocástico.

Proposição 4.53 (Tabela de cálculo). Sejam $\mathbf{B}^{1}, \mathbf{B}^{2}$ movimentos brownianos independentes e defina o processo determinístico $\mathbf{t}$, dado por $t(s, \cdot)=s$, para todo $\omega \in \Omega$ e $s \in[0, \infty)$. Então para $i \in\{1,2\}$,

$$
d B_{s}^{1} d B_{s}^{2}=0, \quad d t_{s} d t_{s}=0, \quad d B_{s}^{i} d t_{s}=0 \quad e d B_{s}^{i} d B_{s}^{i}=d t_{s}
$$

É comum "confundir" a diferencial estocástica $d t_{s}$ pela diferencial newtoniana $d s$ (pois para qualquer processo $\mathbf{H}$ integrável, vale que $\left.\int_{0}^{a} H_{s} d t_{s}=\int_{0}^{a} H_{s} d s\right)$ e portanto as relações acima são comumente utilizadas como na tabela abaixo

\begin{tabular}{rccc}
\hline & $d s$ & $d B_{s}^{1}$ & $d B_{s}^{2}$ \\
\hline$d s$ & 0 & 0 & 0 \\
$d B_{s}^{1}$ & 0 & $d s$ & 0 \\
$d B_{s}^{2}$ & 0 & 0 & $d s$ \\
\hline
\end{tabular}

Demonstração. De fato, como observamos ao introduzir a notação diferencial, dados dois processos estocásticos $\mathbf{M}$ e $\mathbf{N}$, escrever que $d \llbracket \mathbf{M}, \mathbf{N} \rrbracket=d \mathbf{M} d \mathbf{N}=0$ significa que $d \mathbf{M} d \mathbf{N}=0 d \mathbf{X}$ para algum semi-martingal $\mathbf{X}$. Da definição de notação diferencial, isto resulta portanto que $d \mathbf{M} d \mathbf{N}=0$ se, e somente se, para todo $t \geq 0 \llbracket \mathbf{M}, \mathbf{N} \rrbracket_{t}=\llbracket \mathbf{M}, \mathbf{N} \rrbracket_{0}+\int_{0}^{t} 0 d \mathbf{X}=\llbracket \mathbf{M}, \mathbf{N} \rrbracket_{0}$. Agora, pelo teorema de caracterização de Lévy, temos que $\llbracket \mathbf{B}^{\mathbf{1}}, \mathbf{B}^{\mathbf{2}} \rrbracket_{t}=0$, para todo $t \geq 0$ e portanto segue que $d B_{s}^{1} d B_{s}^{2}=d \llbracket \mathbf{B}^{1}, \mathbf{B}^{\mathbf{2}} \rrbracket_{s}=0$. Ainda pelo teorema de caracterização de Lévy, para $i \in\{1,2\}$, 
$d B_{s}^{i} d B_{s}^{i}=d \llbracket \mathbf{B}^{\mathbf{i}}, \mathbf{B}^{\mathbf{i}} \rrbracket_{s}=d \llbracket \mathbf{B}^{\mathbf{i}} \rrbracket_{s}=d \llbracket \mathbf{t} \rrbracket_{s}=d s$. Note também que pela definição 3.5 na página $35, \mathrm{o}$ processo $\mathbf{t}$ é de variação finita e portanto, pela proposição 3.29 na página 58 , item ii) $\llbracket \mathbf{t} \rrbracket_{s}=0$, para todo $s \in[0, \infty)$, logo $d s d s=0$. Finalmente, como para $i \in\{1,2\}, s \mathbf{B}^{i}$ é martingal local, segue pelo corolário 3.17 na página 51 que $\llbracket \mathbf{t}, \mathbf{B}^{\mathbf{i}} \rrbracket_{s}=0$ e portanto $d t_{s} d B_{s}^{i}=d s d B_{s}^{i}=0$. Como queríamos.

\subsubsection{Funções harmônicas e o retorno do movimento Browniano para a origem}

Como mais uma aplicação da fórmula de Itô, vamos mostrar a famosa fórmula de Dynkin para o movimento Browniano. Considere $\mathbf{W}$ um movimento Browniano $d$-dimensional que inicia seu movimento em $x \in \mathbb{R}^{d}$, mais precisamente, $\mathbf{W}$ admite a representação $\mathbf{W}=x+\mathbf{B}$, onde $\mathbf{B}$ é o movimento Browniano padrão $d$-dimensional que começa na origem.

Proposição 4.54. Sejam $\mathbf{W}$ um movimento Browniano d-dimensional iniciando em $x \in \mathbb{R}^{d}, U$ um aberto de $\mathbb{R}^{d}$ e $f \in \mathcal{C}^{2}(U)$ harmônica em $U$, isto é,

$$
\sum_{i=1}^{d} \frac{\partial^{2} f}{\partial x_{i}^{2}}=0
$$

Além disso, suponha que $\tau$ é um tempo de parada finito e que as trajétorias de $\mathbf{W}$ no intervalo [0, $\tau]$ fiquem em um compacto contido em U. Então,

$$
\mathbf{E}(f(W(\tau)))=f(x)
$$

Demonstração. Pela fórmula de Itô, temos

$$
\begin{aligned}
f\left(\mathbf{W}^{\tau}\right)-f\left(W_{0}\right) & =f\left(\mathbf{W}^{\tau}\right)-f(x) \\
& =\sum_{i=1}^{d} D_{i} f\left(\mathbf{W}^{\tau}\right) \bullet\left(\mathbf{W}^{(i)}\right)^{\tau}+\frac{1}{2} \sum_{i, j=1}^{d} D_{i j} f\left(\mathbf{W}^{\tau}\right) \bullet \llbracket\left(\mathbf{W}^{(i)}\right)^{\tau},\left(\mathbf{W}^{(j)}\right)^{\tau} \rrbracket .
\end{aligned}
$$

Pela caracterização de Lévy, observe que $\llbracket\left(\mathbf{W}^{(i)}\right)^{\tau},\left(\mathbf{W}^{(j)}\right)^{\tau} \rrbracket=0$ para $i \neq j$ e $\llbracket\left(\mathbf{W}^{(i)}\right)^{\tau} \rrbracket_{s}=s \wedge \tau$. Dessa observação e pelo fato de $f$ ser harmônica, (4.36) fica 


$$
\begin{aligned}
f\left(\mathbf{W}^{\tau}\right)-f(x) & =\sum_{i=1}^{d} D_{i} f\left(\mathbf{W}^{\tau}\right) \bullet\left(\mathbf{W}^{(i)}\right)^{\tau}+\frac{1}{2} \sum_{i}^{d} D_{i j} f\left(\mathbf{W}^{\tau}\right) \bullet \llbracket\left(\mathbf{W}^{(i)}\right)^{\tau} \rrbracket \\
& =\sum_{i=1}^{d} D_{i} f\left(\mathbf{W}^{\tau}\right) \bullet\left(\mathbf{W}^{(i)}\right)^{\tau}=\sum_{i=1}^{d} \int_{0}^{t} D_{i} f\left(W_{s}^{\tau}\right) d\left(W_{s}^{(i)}\right)^{\tau} .
\end{aligned}
$$

Agora, por hipótese, os integrandos das integrais acima são funções contínuas e limitadas, e portanto cada integral estocástica acima é um martingal. Assim, tomando o valor esperado dos dois lados de (4.37) temos para todo $t \in[0, \infty)$

$$
\mathbf{E}\left(f\left(W_{t}^{\tau}\right)-f(x)\right)=0 .
$$

Como as trajétorias de $\mathbf{W}$ são limitadas no intervalo $[0, \tau]$, podemos usar o teorema da convergência dominada e assim, fazendo $t \rightarrow \infty$ na última equação resulta a fórmula de Dynkin (4.35).

Considere novamente $\mathbf{W}$ o movimento Browniano $d$-dimensional que começa em $x \in \mathbb{R}^{d} \backslash\{0\}$ e defina $T \doteq \inf _{t \geq 0}\left\{\left\|W_{t}\right\|\right\}$. Vamos estudar a distribuição de $T$. Para isso, suponha que $\|x\| \in A$ onde $A \doteq\left\{y \in \mathbb{R}^{d}: r \leq\|y\| \leq R\right\}$. Usando o fato de que as trajétorias de um movimento Browniano são continuas e ilimitadas em $[0, \infty)$ para quase todo $\omega \in \Omega$, então as trajetórias de $\mathbf{W}$ quase sempre saem de $A$. Agora, é sabido que $f$ definida no aberto $U=\mathbb{R}^{d} \backslash\{0\}$ por

$$
f(x) \doteq \begin{cases}\log (\|x\|) & \text { se } d=2 \\ \|x\|^{2-d} & \text { se } d \geq 3\end{cases}
$$

é harmônica. Assim, definindo $\tau \doteq \inf _{t \geq 0}\left\{W_{t} \in \partial A\right\}$. Para $d=2$, temos então, pela fórmula de Dynkin que

$$
\mathbf{E}\left(f\left(W_{\tau}\right)\right)=\log (\|x\|) .
$$

Como não sabemos se $\mathbf{W}$ primeiro cruza $\partial A$ pela fronteira de dentro ou pela fronteira de fora, segue que

$$
\mathbf{E}\left(f\left(W_{\tau}\right)\right)=\log (r) \mathrm{P}\left(\left\|W_{\tau}\right\|=r\right)+\log (R)\left(1-\mathrm{P}\left(\left\|W_{\tau}\right\|=r\right)\right)
$$


e portanto dessas últimas duas equações resulta que

$$
\mathrm{P}\left(\left\|W_{\tau}\right\|=r\right)=\frac{\log (\|x\|)-\log (R)}{\log (r)-\log (R)} .
$$

Assim, se fizermos $R \rightarrow \infty$, obtemos a probabilidade de $\mathbf{W}$ cruzar a bola de raio $r \leq\|x\|$. Como $\lim _{R \rightarrow \infty} \log (R)=\infty$ temos que

$$
\lim _{R \rightarrow \infty} \mathrm{P}\left(\left\|W_{\tau}\right\|=r\right)=1,
$$

e portanto com probabilidade 1 as trajetórias do processo $\mathbf{W}$ cruzam a bola de raio $r$. Agora, fixando $R$ e fazendo $r \rightarrow 0$, temos que

$$
\lim _{r \rightarrow 0} \mathrm{P}\left(\left\|W_{\tau}\right\|=r\right)=0 .
$$

Disso resulta que com probabilidade 0 , as trajetórias de $\mathbf{W}$ passam pela origem antes de sair da bola de raio $R$. Da arbitrariedade de $R$ segue que a probabilidade das trajetórias de $\mathbf{W}$ serem a função nula é 0 .

Agora, analisando a situação para $d \geq 3$ temos que

$$
\mathrm{P}\left(\left\|W_{\tau}\right\|=r\right)=\frac{\|x\|^{2-d}-R^{2-d}}{r^{2-d}-R^{2-d}},
$$

e como $\lim _{R \rightarrow \infty} R^{2-d}=0$, segue que a probabilidade de $\mathbf{W}$ cruzar a bola de raio $r \leq\|x\|$ é

$$
\lim _{R \rightarrow \infty} \mathrm{P}\left(\left\|W_{\tau}\right\|=r\right)=\left(\frac{r}{\|x\|}\right)^{d-2}
$$

Então, assim como no caso anterior, a probabilidade das trajetórias de $\mathbf{W}$ serem a função nula é 0 .

Perceba que só analisamos os casos no qual o movimento Browniano se inicia em um ponto diferente da origem. Será que se ele começar na origem pode ficar lá para sempre? A resposta é não. De fato, suponha que $x=0$ e seja $\varepsilon>0$. Vamos mostrar que $\mathrm{P}\left(\left\|W_{t}\right\|>0, \forall t>0\right)=1$. Para isso, note que $\mathrm{P}\left(\left\|W_{t}\right\|>0, \forall t>0\right)=\lim _{\varepsilon \rightarrow 0} \mathrm{P}\left(\inf _{t \geq \varepsilon}\left\|W_{t}\right\|>0\right)$. Porém,

$$
\mathrm{P}\left(\inf _{t \geq \varepsilon}\left\|W_{t}\right\|>0\right)=\int_{\mathbb{R}^{d}} \mathrm{P}\left(\inf _{t \geq \varepsilon}\left\|W_{t}\right\|>0 \mid W(\varepsilon)=y\right) d \Phi(y)
$$


onde $\Phi(y)$ é a distribuição de $W_{\varepsilon}$. Agora, pelas propriedades do movimento Browniano, temos que

$$
\begin{aligned}
\mathrm{P}\left(\inf _{t \geq \varepsilon}\left\|W_{t}\right\|>0 \mid W(\varepsilon)=y\right) & =\mathrm{P}\left(\inf _{t \geq \varepsilon}\left\|W_{t}-W_{\varepsilon}+y\right\|>0\right) \\
& =\mathrm{P}\left(\inf _{u \geq 0}\left\|W_{u}+y\right\|>0\right) .
\end{aligned}
$$

Assim, temos que

$$
\begin{aligned}
\mathrm{P}\left(\int_{\geq \geq \varepsilon}\left\|W_{t}\right\|>0\right) & =\int_{\mathbb{R}^{d}} \mathrm{P}\left(\inf _{u \geq 0}\left\|W_{u}+y\right\|>0\right) d \Phi(y) \\
& =\int_{\mathbb{R}^{d} \backslash\{0\}} \mathrm{P}\left(\inf _{u \geq 0}\left\|W_{u}+y\right\|>0\right) d \Phi(y) \\
& =\int_{\mathbb{R}^{d} \backslash\{0\}} 1 d \Phi(y)=1 .
\end{aligned}
$$

Aqui usamos o resultado anterior e o fato de que a integral de uma densidade de probabilidade é 1 . Assim,

$$
\mathrm{P}\left(\left\|W_{t}\right\|>0, \forall t>0\right)=\lim _{\varepsilon \rightarrow 0} \mathrm{P}\left(\inf _{t \geq \varepsilon}\left\|W_{t}\right\|>0\right)=1 .
$$

Isso mostra que o movimento Browniano multidimensional, com probabilidade 1, não volta para a origem não importa o seu ponto inicial. 


\section{Capítulo 5}

\section{Conexões entre equações diferenciais estocásticas e par- ciais}

What we know about the global financial crisis is that we don't know very much.

Paul Samuelson

\subsection{Existência e unicidade para equações diferenciais estocásticas}

Equações diferenciais estocásticas são usadas para descrever a dinâmica de processos que dependem de alguma componente aleatória.

O exemplo clássico de equação diferencial estocástica aparece ao modelar o movimento de uma pequena partícula suspensa em um líquido. Caso o movimento da particula se deva apenas ao movimento do líquido, então denotando por $v(t, x)$ o campo de velocidade do líquido e $X_{t}$ a posição da partícula no instante $t \geq 0$, a equação do movimento é dada por $\frac{d X_{t}}{d t}=v\left(t, X_{t}\right)$, ou, de maneira formal $d X_{t}=v\left(t, X_{t}\right) d t$. Observamos que no cálculo usual, uma equação diferencial ordinária como esta pode ser interpretada como uma equação integral $X_{t}=X_{a}+\int_{a}^{t} f(s, X(s)) d s$.

Se considerarmos agora que a posição da partícula muda também por influência das colisões aleatórias com as moléculas do líquido, podemos modelar formalmente a equação de movimento da partícula pela relação

$$
d X_{t}=v\left(t, X_{t}\right) d t+\sigma\left(t, X_{t}\right) d W_{t}
$$


Aqui $\sigma$ é a taxa de colisão da moléculas com a partícula e $\mathbf{W}$ é um movimento Browniano. A equação diferencial estocástica (5.1) é apenas uma expressão simbólica para a equação integral estocástica

$$
X_{t}=X_{a}+\int_{a}^{t} f\left(s, X_{s}\right) d s+\int_{a}^{t} \sigma\left(s, X_{s}\right) d W_{s}
$$

cujo sentido preciso é conhecido devido a teoria de integração estocástica iniciada por K. Itô. Esta seção é dedicada ao estudo do conceito de solução de uma equação diferencial estocástica.

Sejam $b_{i}(t, x), \sigma_{i j}(t, x) ; 1 \leq i \leq d, 1 \leq j \leq r$, funções Borel-mensuráveis de $[0, \infty) \times \mathbb{R}^{d}$ com valores em $\mathbb{R}$. Chamamos o vetor $b(t, x) \doteq\left\{b_{i}(t, x)\right\}_{1 \leq i \leq d}$ de drift e a matriz $\sigma(t, x) \doteq\left\{\sigma_{i j}(t, x)\right\}_{\substack{1 \leq i \leq d \\ 1 \leq j \leq r}}$ de dispersão. Como dito antes, nosso objetivo é apresentar um dos conceitos de solução para equação diferencial estocástica (ede)

$$
d X_{t}=b\left(t, X_{t}\right) d t+\sigma\left(t, X_{t}\right) d W_{t}
$$

que também pode ser escrita na forma componente-a-componente

$$
d X_{t}^{(i)}=b_{i}\left(t, X_{t}\right) d t+\sum_{j=1}^{r} \sigma_{i j}\left(t, X_{t}\right) d W_{t}^{(j)}, \quad 1 \leq i \leq d .
$$

Vamos considerar neste trabalho ede's com respeito a movimentos Brownianos, isto é, $\mathbf{W}=\left\{W_{t}: 0 \leq t<\infty\right\}$ é um movimento Browniano $r$-dimensional. O processo $\mathbf{X}=\left\{X_{t}: 0 \leq t<\infty\right\}$, tem trajétorias contínuas com valores em $\mathbb{R}^{d}$ e será chamado de solução da ede 5.2. Neste trabalho a norma de uma matriz $\sigma,(d \times r)$ é dada por

$$
\|\sigma\|^{2} \doteq \sum_{i=1}^{d} \sum_{j=1}^{r} \sigma_{i j}^{2}
$$

Agora, considere agora um espaço de probabilidade $(\Omega, \mathscr{F}, \mathrm{P})$ e um movimento Browniano $r$ dimensional $\mathbf{W}=\left\{W_{t}, \mathscr{F}_{t}^{\mathbf{W}}, 0 \leq t<\infty\right\}$. Vamos supor também que seja possível definir um vetor aleatório $\xi$ tomando valores em $\mathbb{R}^{d}$ e que seja independente de $\mathscr{F} \mathbf{W}=\sigma\left(\bigcup_{t \geq 0} \mathscr{F}_{t} \mathbf{W}\right)$. A filtração que iremos considerar é construida da seguinte forma: Consideremos $\left\{\mathscr{G}_{t}\right\}$, a filtração gerada conjuntamente por $\xi$ e $\mathbf{W}$, isto é,

$$
\mathscr{G}_{t} \doteq \sigma\left(\xi, W_{s} ; 0 \leq s \leq t\right), \quad t \in[0, \infty[
$$


e seja

$$
\mathcal{N} \doteq\left\{N \subseteq \Omega: \exists G \in \sigma\left(\bigcup_{t \geq 0} \mathscr{G}_{t}\right) \text { tal que } N \subseteq G \text { e } \mathrm{P}(G)=0\right\} .
$$

Agora, tomamos a filtração aumentada

$$
\mathscr{F}_{t} \doteq \sigma\left(\mathscr{G}_{t} \cup \mathcal{N}\right), \quad t \in[0, \infty)
$$

Temos que com esta filtração, o espaço $\left(\Omega, \mathscr{F},\left\{\mathscr{F}_{t}\right\}, \mathrm{P}\right)$ satisfaz as condições usuais*.

Definição 5.1. Dado o espaço $(\Omega, \mathscr{F}, \mathrm{P})$ definido acima, dizemos que uma solução forte da ede (5.2), com respeito ao movimento Browniano $\mathbf{W}$ previamente fixado e a condição inicial $\xi$, é um processo estocástico $\mathbf{X}$ com trajétorias contínuas tal que

i) $\mathbf{X}$ é adaptado à filtração (5.3),

ii) $\mathrm{P}\left(X_{0}=\xi\right)=1$,

iii) $\int_{0}^{t} b_{i}\left(s, X_{s}\right)+\sigma_{i j}^{2}\left(s, X_{s}\right) d s<\infty$, P-q.s., para todo $1 \leq i \leq d, 1 \leq j \leq r$ e $t \in[0, \infty)$

iv)

$$
X_{t}=X_{0}+\int_{0}^{t} b\left(s, X_{s}\right) d s+\int_{0}^{t} \sigma\left(s, X_{s}\right) d W_{s}, \quad t \in[0, \infty), \mathrm{P}-\mathrm{q} . \mathrm{s} .
$$

ou, de forma equivalente,

$$
X_{t}^{(i)}=X_{0}^{(i)}+\int_{0}^{t} b_{i}\left(s, X_{s}\right) d s+\sum_{j=1}^{r} \int_{0}^{t} \sigma_{i j}\left(s, X_{s}\right) d W_{s}^{(j)}, \quad t \in[0, \infty), 1 \leq i \leq d, \mathrm{P}-\mathrm{q} . \mathrm{s} .
$$

Observamos que no caso de soluções fortes, o movimento Browniano W é um dado do problema. Esta é a principal diferença quando consideramos as chamadas soluções fracas de uma ede. Neste trabalho não estudaremos soluções fracas. Uma refêrencia para o assunto é [23]. Vamos agora tratar das questões de existência e unicidade de soluções fortes para edes. Começaremos definindo formalmente o que entendemos por unicidade.

\footnotetext{
* As condições usuais foram introduzidas na definição 2.24 na página 17. A prova da afirmação pode ser encontrada por exemplo em [23], capítulo 2.7 .
} 
Definição 5.2. Dizemos que vale a unicidade forte de soluções para a ede (5.2), quando para qualquer movimento Browniano $\mathbf{W}$ r-dimensional e $\mathbf{X}, \mathbf{Y}$ duas soluções fortes de (5.2) valer que $\mathrm{P}\left(X_{t}=Y_{t}, 0 \leq t<\infty\right)=1$, ou seja, $\mathbf{X}$ e $\mathbf{Y}$ são indistinguíveis.

Trataremos agora de questões de existência e unicidade. Como nota histórica, o primeiro a obter tais resultados foi K. Itô. Antes, precisaremos de um resultado clássico cuja demonstração pode ser encontrada, por exemplo em [13].

Lema 5.3 (Desigualdade de Gronwall). Seja $\phi$ uma função contínua tal que

$$
\phi(t) \leq f(t)+\beta \int_{0}^{t} \phi(s) d s, \quad \forall t \in[0, T]
$$

onde $f \in \mathrm{L}^{1}[0, T]$ e $\beta$ é uma constante positiva. Então,

$$
\phi(t) \leq f(t)+\beta \int_{0}^{t} f(s) e^{\beta(t-s)} d s .
$$

Em particular, quando $f$ é uma constante $\alpha$, temos

$$
\phi(t) \leq \alpha e^{\beta(t)}, \quad \forall 0 \leq t \leq T .
$$

Teorema 5.4. Suponha que os coeficientes $b(t, x), \sigma(t, x)$ sejam localmente Lipschitz-contínuos na variável espacial, isto é, para cada inteiro $n \geq 1$, existe uma constante $K_{n}>0$ tal que para quaisquer $t \geq 0,\|x\| \leq n e\|y\| \leq n$, vale que

$$
\|b(t, x)-b(t, y)\|+\|\sigma(t, x)-\sigma(t, y)\| \leq K_{n}\|x-y\|
$$

Então vale a unicidade forte para a ede (5.2).

Demonstração. Sejam $\mathbf{X}$ e $\mathbf{Y}$ duas soluções fortes da ede (5.2) definidas para $t \geq 0$, com respeito ao mesmo movimento Browniano $\mathbf{W}$ e condição inicial $\xi$ em $(\Omega, \mathscr{F}, \mathrm{P})$. Para provar que $\mathbf{X}$ e $\mathbf{Y}$ são indistinguíveis, pela continuidade dos processos, basta mostrar que um é modificação do outro*. Para isso, é suficiente mostrar que $\mathbf{E}\left(\left\|X_{t}-Y_{t}\right\|^{2}\right)=0$, para todo $t \geq 0$. Assim, começamos definindo os

* Veja a proposição 2.3 na página 6 . 
tempos de parada $\tau_{n} \doteq \inf \left\{t \geq 0 ;\left\|X_{t}\right\| \geq n\right\}, \eta_{n} \doteq \inf \left\{t \geq 0 ;\left\|Y_{t}\right\| \geq n\right\}$ e $\rho_{n} \doteq \tau_{n} \wedge \eta_{n}$. Agora para $t \in[0, T]$, temos que

$$
\begin{aligned}
\mathbf{E}\left(\left\|X_{t \wedge \rho_{n}}-Y_{t \wedge \rho_{n}}\right\|^{2}\right) & =\mathbf{E}\left(\left\|\int_{0}^{t \wedge \rho_{n}}\left[b\left(u, X_{u}\right)-b\left(u, Y_{u}\right)\right] d u+\int_{0}^{t \wedge \rho_{n}}\left[\sigma\left(u, X_{u}\right)-\sigma\left(u, Y_{u}\right)\right] d W_{u}\right\|^{2}\right) \\
& \leq \mathbf{E}\left(4\left\|\int_{0}^{2}\left[b\left(u, X_{u}\right)-b\left(u, Y_{u}\right)\right] d u\right\|^{2}\right) \\
& +\mathbf{E}\left(4\left\|\int_{0}^{t \wedge \rho_{n}}\left[\sigma\left(u, X_{u}\right)-\sigma\left(u, Y_{u}\right)\right] d W_{u}\right\|^{2}\right) \\
& \leq 4 t \mathbf{E}\left(\int_{0}^{t \wedge \rho_{n}}\left\|b\left(u, X_{u}\right)-b\left(u, Y_{u}\right)\right\|^{2} d u\right) \\
& +4 \mathbf{E}\left(\int_{0}^{t \wedge \rho_{n}}\left\|\sigma\left(u, X_{u}\right)-\sigma\left(u, Y_{u}\right)\right\|^{2} d u\right) \\
& \leq 4(T+1) K_{n}^{2} \int_{0}^{t} \mathbf{E}\left(\left\|X_{u \wedge \rho_{n}}-Y_{u \wedge \rho_{n}}\right\|^{2}\right) d u .
\end{aligned}
$$

Aqui, para a primeira desigualdade usamos o fato que $\left\|v_{1}+\cdots v_{k}\right\|^{2} \leq k^{2}\left(\left\|v_{1}\right\|^{2}+\cdots+\left\|v_{k}\right\|^{2}\right)$. Para a segunda, usamos a desigualdade de Hölder e a isometria de Itô ${ }^{*}$. Para obter a última desigualdade, usamos (5.4), o fato que $t \in[0, T]$ e o teorema de Fubini. Pela desigualdade de Gronwall (onde $\mathbf{E}\left(\left\|X_{t \wedge \rho_{n}}-Y_{t \wedge \rho_{n}}\right\|^{2}\right)$, faz o papel de $\left.\phi(t)\right)$ segue que os processos $\left\{X_{t \wedge \rho_{n}} ; 0 \leq t<\infty\right\}$ e $\left\{Y_{t \wedge \rho_{n}} ; 0 \leq t<\infty\right\}$ são modificações um do outro e portanto são indistinguiveis. Fazendo $n \rightarrow \infty$, pelo fato de $\lim _{n \rightarrow \infty} \rho_{n}=\infty$, P-q. s., o mesmo vale para $\left\{X_{t} ; 0 \leq t<\infty\right\}$ e $\left\{Y_{t} ; 0 \leq t<\infty\right\}$. Como queríamos.

Assim como no caso de equações diferenciais ordinárias, podemos ter soluções definidas não

\footnotetext{
* Veja o teorema 4.17 na página 76.
} 
globalmente como é o caso da equação

$$
\frac{d X_{t}}{d t}=X_{t}^{2}, \quad X_{0}=1
$$

Nesse caso, $\sigma=0$ e $b(x)=x^{2}$. A única solução é dada por $X_{t}=\frac{1}{1-t}$ mas só está definida para $t \in$ $[0,1)$. De maneira mais geral, o próximo teorema apresenta condições para que tenhamos existência de soluções globais de equações diferenciais estocásticas que elimine a possibilidade de explosões como no exemplo acima. Antes vamos enunciar um lema cuja demonstração pode ser encontrada em [23], página 166.

Lema 5.5. Seja $\mathbf{M}=\left(\mathbf{M}^{(1)}, \ldots, \mathbf{M}^{(d)}\right)$ um vetor de martingais locais contínuos dados por

$$
M_{t}^{(i)}=\sum_{j=1}^{r} \int_{0}^{t} \sigma_{i j}(s, \omega) d W_{s}^{(j)}
$$

onde $\mathbf{W}$ é um movimento Browniano padrão $r$-dimensional e $\sigma_{i j}$ é uma função mensurável e adaptada para cada $1 \leq i \leq d$ e $1 \leq j \leq r$. Então existe uma constante positiva $\kappa$ tal que para qualquer tempo de parada $\tau$, vale que

$$
\mathbf{E}\left(\max _{0 \leq s \leq \tau}\left\|M_{s}\right\|^{2}\right) \leq \kappa \mathbf{E}\left(\int_{0}^{\tau}\|\sigma(t)\|^{2} d t\right) .
$$

Teorema 5.6. Suponha que os coeficientes b e $\sigma$ satisfaçam

$$
\|b(t, x)-b(t, y)\|+\|\sigma(t, x)-\sigma(t, y)\| \leq K\|x-y\|
$$

$e$

$$
\|b(t, x)\|^{2}+\|\sigma(t, x)\|^{2} \leq K^{2}\left(1+\|x\|^{2}\right),
$$

para todo $0 \leq t<\infty, x, y \in \mathbb{R}^{d}$ e $K$ é uma constante positiva. Considere o espaço de probabilidade, $\xi e$ $\mathbf{W}$ como apresentados acima da definição 5.1. Além disso suponha que $\mathbf{E}\left(\|\xi\|^{2}\right)<\infty$. Então existe um processo $\mathbf{X}$ contínuo e adaptado à filtração (5.3) que é solução forte da ede (5.2) com respeito ao movimento Browniano $\mathbf{W}$ e a condição inicial $\xi$. Além disso, esse processo é de quadrado integrável. De maneira mais precisa, para cada $T>0$, existe uma constante $C$ que depende apenas de $K$ e $T$ tal que

$$
\mathbf{E}\left(\left\|X_{t}\right\|^{2}\right) \leq C\left(1+\mathbf{E}\left(\|\xi\|^{2}\right)\right) e^{C t}, \quad t \in[0, T] .
$$


Demonstração. Defina $X_{t}^{(0)} \doteq \xi$ e para $k \geq 0$

$$
X_{t}^{(k+1)} \doteq \xi+\int_{0}^{t} b\left(s, X_{s}^{(k)}\right) d s+\int_{0}^{t} \sigma\left(s, X_{s}^{(k)}\right) d W_{s}, \quad t \in[0, \infty)
$$

Para cada $T \geq 0$, vamos provar por indução que para todo $k \in\{0,1, \ldots\},, \mathbf{X}^{(k)}$ é um processo

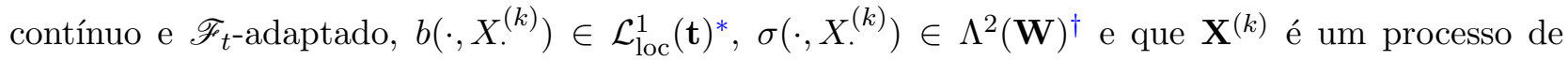
quadrado integrável, em particular

$$
\mathbf{E}\left(\left\|X_{t}^{(k)}\right\|^{2}\right) \leq C\left(1+\mathbf{E}\left(\|\xi\|^{2}\right)\right) e^{C t}, \quad 0 \leq t \leq T .
$$

De fato, para $k=0$ é evidente que $\mathbf{X}^{(0)}$ é de quadrado integrável, contínuo e adaptado. Além disso, como $\sigma(\cdot, \cdot)$ é contínuo (pois é Lipschitz), $\mathbf{X}^{(0)}$ é contínuo e adaptado, segue que $\sigma\left(\cdot, X^{(0)}\right.$ ) é contínuo e adaptado e portanto é progressivamente mensurável. Agora

$$
\begin{aligned}
\mathbf{E}\left(\int_{0}^{t}\left\|\sigma\left(s, X_{s}^{(0)}\right)\right\|^{2} d s\right) & \leq \mathbf{E}\left(\int_{0}^{t} K^{2}\left(1+\left\|X_{s}^{(0)}\right\|^{2}\right) d s\right) \\
& <\infty
\end{aligned}
$$

Isso mostra que $\sigma\left(\cdot, X^{(0)}\right) \in \Lambda(\mathbf{W})$. Finalmente, temos também que $b\left(\cdot, X^{(0)}\right)$ é progressivamente mensurável e

$$
\begin{aligned}
\int_{0}^{t}\left\|b\left(s, X_{s}^{(0)}\right)\right\|^{2} d s & \leq K^{2} \int_{0}^{t}\left(1+\left\|X_{s}^{(0)}\right\|^{2}\right) d s \\
& <\infty
\end{aligned}
$$

Como o espaço de medida é finito, isso implica que $\int_{0}^{t}\left\|b\left(s, X_{s}^{(0)}\right)\right\| d s<\infty$, ou seja $b\left(\cdot, X^{(0)}\right) \in \mathcal{L}_{\text {loc }}^{1}$.

Agora, suponha que a afirmação seja válida para algum $k \geq 1$, natural. Temos como na demon-

\footnotetext{
* Aqui t é o processo deterministico, definido como $\mathbf{t}(t)=t, t \geq 0$. É um processo contínuo e de variação finita.

† Veja a definição 4.30 na página 90.
} 
stração do teorema anterior que

$$
\begin{aligned}
\left\|X_{t}^{(k+1)}\right\|^{2} & \leq 9\|\xi\|^{2}+9\left\|\int_{0}^{t} b\left(s, X_{s}^{(k)}\right) d s\right\|^{2}+9\left\|\int_{0}^{t} \sigma\left(s, X_{s}^{(k)}\right) d W_{s}\right\|^{2} \\
& \leq 9\|\xi\|^{2}+9 t \int_{0}^{t}\left\|b\left(s, X_{s}^{(k)}\right)\right\|^{2} d s+9\left\|\int_{0}^{t} \sigma\left(s, X_{s}^{(k)}\right) d W_{s}\right\|^{2} .
\end{aligned}
$$

Assim, pela isometria de Itô e linearidade da esperança,

$$
\begin{aligned}
\mathbf{E}\left(\left\|X_{t}^{(k+1)}\right\|^{2}\right) & \leq 9 \mathbf{E}\left(\|\xi\|^{2}\right)+9 t \mathbf{E}\left(\int_{0}^{t} \| b\left(s, X_{s}^{(k)} \|^{2} d s\right)+9 \mathbf{E}\left(\int_{0}^{t}\left\|\sigma\left(s, X_{s}^{(k)}\right)\right\|^{2} d s\right)\right. \\
& \leq 9 \mathbf{E}\left(\|\xi\|^{2}\right)+9 T \mathbf{E}\left(\int_{0}^{t} K^{2}\left(1+\left\|X_{s}^{(k)}\right\|^{2}\right) d s\right)+9 \mathbf{E}\left(\int_{0}^{t} K^{2}\left(1+\left\|X_{s}^{(k)}\right\|^{2}\right) d s\right) \\
& \leq 9 \mathbf{E}\left(\|\xi\|^{2}\right)+9 K^{2}(T+1) \int_{0}^{t} 1+\mathbf{E}\left(\left\|X_{s}^{(k)}\right\|^{2}\right) d s .
\end{aligned}
$$

Definindo $C \doteq 9(T+1) K^{2}$, temos que

$$
\mathbf{E}\left(\left\|X_{t}^{(k+1)}\right\|^{2}\right) \leq C\left(1+\mathbf{E}\left(\|\xi\|^{2}\right)\right)+C \int_{0}^{t} \mathbf{E}\left(\left\|X_{s}^{(k)}\right\|^{2}\right) d s<\infty \quad t \in[0, T],
$$

e isso prova que $\mathbf{X}^{(k+1)}$ é de quadrado integrável. Agora, assim como no caso $k=0, \sigma\left(\cdot, X^{k+1}\right)$ é progressivamente mensurável e como

$$
\begin{aligned}
\mathbf{E}\left(\int_{0}^{t}\left\|\sigma\left(s, X_{s}^{(k+1)}\right)\right\|^{2} d s\right) & \leq \mathbf{E}\left(\int_{0}^{t} K^{2}\left(1+\left\|X_{s}^{(k+1)}\right\|^{2}\right) d s\right) \\
& <\infty
\end{aligned}
$$

temos que $\sigma\left(\cdot, X^{(k+1)}\right) \in \Lambda^{2}(\mathbf{W})$ e portanto $\int_{0}^{t} \sigma\left(s, X_{s}^{(k+1)}\right) d W_{s}$ é um martingal contínuo e adaptado. 
Segue também que $b\left(\cdot, X^{(0)}\right)$ é progressivamente mensurável e

$$
\begin{aligned}
\int_{0}^{t}\left\|b\left(s, X_{s}^{(k+1)}\right)\right\|^{2} d s & \leq K^{2} \int_{0}^{t}\left(1+\left\|X_{s}^{(k+1)}\right\|^{2}\right) d s \\
& <\infty .
\end{aligned}
$$

Como o espaço de medida é finito, isso implica que $\int_{0}^{t}\left\|b\left(s, X_{s}^{(k+1)}\right)\right\| d s<\infty$, ou seja $b\left(\cdot, X^{(k+1)}\right) \in \mathcal{L}_{\text {loc }}^{1}$ e portanto pela proposição 4.4 na página 65 , segue que $\int_{0}^{t} b\left(s, X_{s}^{(k+1)}\right) d s$ é contínuo e adaptado. Disso resulta que $\mathbf{X}^{(k+1)}$ é um processo contínuo e adaptado. Finalmente, iterando (5.10) ficamos com

$$
\mathbf{E}\left(\left\|X_{t}^{(k+1)}\right\|^{2}\right) \leq C\left(1+\mathbf{E}\left(\|\xi\|^{2}\right)\right)\left(1+C t+\frac{(C t)^{2}}{2 !}+\cdots+\frac{(C t)^{k+1}}{(k+1) !}\right),
$$

e portanto (5.9) vale.

Agora defina

$$
B_{t} \doteq \int_{0}^{t}\left\{b\left(s, X_{s}^{(k)}\right)-b\left(s, X_{s}^{(k-1)}\right)\right\} d s, M_{t} \doteq \int_{0}^{t}\left\{\sigma\left(s, X_{s}^{(k)}\right)-\sigma\left(s, X_{s}^{(k-1)}\right)\right\} d W_{s}
$$

Temos que $X_{t}^{(k+1)}-X_{t}^{(k)}=B_{t}-M_{t}$. Pelo lema anterior e por hipótese, temos que

$$
\begin{aligned}
\mathbf{E}\left(\max _{0 \leq s \leq t}\left\|M_{s}\right\|^{2}\right) & \leq \kappa \mathbf{E}\left(\int_{0}^{t}\left\|\sigma\left(s, X_{s}^{(k)}\right)-\sigma\left(s, X_{s}^{(k-1)}\right)\right\|^{2}\right) d s \\
& \leq \kappa K^{2} \mathbf{E}\left(\int_{0}^{t}\left\|X_{s}^{(k)}-X_{s}^{(k-1)}\right\|^{2} d s\right) .
\end{aligned}
$$

Por outro lado, usando a hipótese e a desigualdade de Schwarz, temos que

$$
\mathbf{E}\left(\left\|B_{t}\right\|^{2}\right) \leq K^{2} t \int_{0}^{t} \mathbf{E}\left(\left\|X_{s}^{(k)}-X_{s}^{(k-1)}\right\|^{2}\right) d s
$$


e assim

$$
\mathbf{E}\left(\max _{0 \leq s \leq t}\left\|X_{s}^{(k+1)}-X_{s}^{(k)}\right\|^{2}\right) \leq\left(4 K^{2}(\kappa+T)\right) \int_{0}^{t} \mathbf{E}\left(\left\|X_{s}^{(k+1)}-X_{s}^{(k)}\right\|^{2}\right) d s, \quad 0 \leq t \leq T .
$$

Iterando essa última desigualdade, ficamos com

$$
\mathbf{E}\left(\max _{0 \leq s \leq t}\left\|X_{s}^{(k+1)}-X_{s}^{(k)}\right\|^{2}\right) \leq \frac{C^{*}(L t)^{k}}{k !}, \quad 0 \leq t \leq T
$$

aqui $L \doteq 4 K^{2}(\kappa+T)$ e $C^{*} \doteq \max _{0 \leq s \leq T}\left\|X_{s}^{(1)}-\xi\right\|^{2}$. Agora, pela desigualdade de Chebyshev* temos para cada $k \geq 1$,

$$
\mathrm{P}\left(\max _{0 \leq t \leq T}\left\|X_{t}^{(k+1)}-X_{t}^{(k)}\right\|^{2}>\frac{1}{2^{k+1}}\right) \leq 4 C^{*} \frac{(4 L T)^{k}}{k !} .
$$

Como $\sum_{k \geq 1} 4 C^{*} \frac{(4 L T)^{k}}{k !}<\infty$, pelo lema de Borel-Cantelli temos que existe um conjunto $\Omega^{*} \in \mathscr{F}$ tal que $\mathrm{P}\left(\Omega^{*}\right)=1$ e uma variável aleatória $N(\omega)$ tal que para $\omega \in \Omega^{*}$

$$
\max _{0 \leq t \leq T}\left\|X_{t}^{(k+m)}(\omega)-X_{t}^{(k)}(\omega)\right\| \leq 2^{-k}, \quad \forall m \geq 1, k \geq N(\omega)
$$

Assim, temos que a sequência de trajetórias $\left\{X_{t}^{(k)}(\omega) ; 0 \leq t \leq T\right\}_{k \geq 1}$ é convergente na norma do supremo das funções contínuas, disso temos a existência de uma trajetória limite contínua $\left\{X_{t}(\omega) ; 0 \leq t \leq T\right\}$ para cada $\omega \in \Omega^{*}$. Da arbitrariedade de $T \geq 0$, temos a existência de um processo contínuo $\mathbf{X}=\left\{X_{t} ; 0 \leq t<\infty\right\}$ com a propriedade de que para quase todo $\omega$ as trajetórias $\left\{X^{(k)}(\omega)\right\}_{k \geq 1}$ convergem para $X .(\omega)$ uniformemente em compactos de [0, $\left.\infty\right)$. Para provar (5.7), basta usar (5.9) e o lema de Fatou. De fato

$$
\mathbf{E}\left(\left\|X_{t}\right\|^{2}\right)=\mathbf{E}\left(\liminf \left\|X_{t}^{(k)}\right\|^{2}\right) \leq \liminf \mathbf{E}\left(\left\|X_{t}^{(k)}\right\|^{2}\right) \leq C\left(1+\mathbf{E}\left(\|\xi\|^{2}\right)\right) e^{C t} .
$$

Por construção temos que $\mathbf{X}$ satisfaz as primeiras três propriedades de uma solução forte (definição 5.1). Para ver que $\mathbf{X}$ satisfaz a última propriedade, vamos tomar o limite para $k \rightarrow \infty$ e ver que as integrais em (5.8) convergem para os limites esperados. De fato, para $T>0$, temos que

\footnotetext{
* Veja o corolário 2.32 na página 19.
} 
$\max _{0 \leq t \leq T}\left\|X_{t}(\omega)-X_{t}^{(k)}(\omega)\right\| \leq 2^{-k}$, para todo $k \geq N(\omega)$. Assim, para $k$ suficientemente grande

$$
\begin{aligned}
\left\|\int_{0}^{t} b\left(s, X_{s}^{(k)}\right) d s-\int_{0}^{t} b\left(s, X_{s}\right) d s\right\|^{2} & \leq\left(\int_{0}^{t}\left\|b\left(s, X_{s}^{(k)}\right)-b\left(s, X_{s}\right)\right\| d s\right)^{2} \\
& \left.\leq\left(\int_{0}^{t} 1 d s\right)^{\frac{1}{2}}\left(\int_{0}^{t}\left\|b\left(s, X_{s}^{(k)}\right)-b\left(s, X_{s}\right)\right\|^{2} d s\right)^{\frac{1}{2}}\right)^{2} \\
& \leq K^{2} T \int_{0}^{T}\left\|X_{s}^{(k)}-X_{s}\right\|^{2} d s \\
& \leq K^{2} T^{2} 2^{-2 k} .
\end{aligned}
$$

Ou seja, $\int_{0}^{t} b\left(s, X_{s}^{(k)}\right) d s \stackrel{\text { q.s }}{\longrightarrow} \int_{0}^{t} b\left(s, X_{s}\right) d s$. Agora, de (5.11) temos que para cada $t \in[0, T]$ fixo, a sequência $\left\{X_{t}^{(k)}\right\}_{k \geq 1}$ é de Cauchy em $\mathrm{L}^{2}(\Omega, \mathscr{F}, \mathrm{P})$ e como $X_{t}^{(k)} \stackrel{\text { q.s }}{\longrightarrow} X_{t}$ temos que $\mathbf{E}\left(\left\|X_{t}^{(k)}-X_{t}\right\|^{2}\right) \rightarrow$ 0 , para $k \rightarrow \infty$. Além disso, vimos que para todo $k \geq 0, \mathbf{E}\left(\left\|X_{t}^{(k)}\right\|^{2}\right)$ e (por Fatou) $\mathbf{E}\left(\left\|X_{t}\right\|^{2}\right)$ são uniformemente limitados. Agora, como

$$
\mathbf{E}\left(\left\|X_{s}^{(k)}-X_{s}\right\|^{2}\right) \leq \mathbf{E}\left(\left(\left\|X_{s}^{(k)}\right\|+\left\|X_{s}\right\|\right)^{2}\right) \leq \mathbf{E}\left(2\left\|X_{s}^{(k)}\right\|^{2}+2\left\|X_{s}\right\|^{2}\right),
$$

temos que $\mathbf{E}\left(\left\|X_{s}^{(k)}-X_{s}\right\|^{2}\right)$ também é uniformemente limitado. Assim, de (5.5) e do teorema da convergência limitada temos que

$$
\begin{aligned}
\mathbf{E}\left(\left\|\int_{0}^{t} \sigma\left(s, X_{s}^{(k)}\right) d W_{s}-\int_{0}^{t} \sigma\left(s, X_{s}\right) d W_{s}\right\|^{2}\right) & =\mathbf{E}\left(\int_{0}^{t}\left\|\sigma\left(s, X_{s}^{(k)}\right)-\sigma\left(s, X_{s}\right)\right\|^{2} d s\right) \\
& \leq K^{2} \int_{0}^{t} \mathbf{E}\left(\left\|X_{s}^{(k)}-X_{s}\right\|^{2}\right) d s \rightarrow 0, \quad \text { quando } k \rightarrow \infty .
\end{aligned}
$$

Isso termina a prova do teorema.

Exemplo 5.7 (Movimento Browniano geométrico). Nesse exemplo vamos estudar um modelo para 
o comportamento do preço de uma ação no mercado financeiro. Considere então um espaço de probabilidade $(\Omega, \mathscr{F}, \mathrm{P})$ que satisfaça as condições usuais e seja $\mathbf{W}$ um movimento Browniano unidimensional. Vamos denotar por $\mathbf{S}$ o processo que descreve os preços da ação. Sendo $S_{t}$ o preço no instante $t>0$, o economista Paul A. Samuelson, refinando as idéias de Louis Bachelier e usando a teoria de integração estocástica de K. Itô, propôs, nos meados do século vinte, um modelo para o retorno do preço da ação, denotado formalmente por $\frac{d S_{t}}{S_{t}}$. De acordo com Samuelson, o retorno da ação poderia ser decomposto como a soma de dois componentes. Um componente sistemático e um aleatório. O componente sistemático consiste no fator $\mu d t$ onde $\mu$ é uma constante que representa a média da taxa de retorno da ação e $d t$ é o pequeno incremento no intervalo de tempo que estamos considerando para estudar o retorno. Na literatura de finanças, $\mu$ é chamado de drit. Já a componente aleatória pode ser modelada como $\sigma d W_{t}$ onde $d W_{t}$ representa as perturbações aleatórias no preço da ação, isto é, uma medida de quanto o preço do ativo flutua e $\sigma$ uma medida da intensidade destas pertubações. A constante $\sigma$ determina o quão volátil o preço do ativo é, e por isso é chamada de volatilidade. Dessa forma, temos a expressão matématica para o modelo de Samuelson:

$$
d S_{t}=S_{t}\left(\mu d t+\sigma d W_{t}\right)
$$

De acordo com a teoria desenvolvida neste trabalho, sabemos que a expressão acima pode ser entendida de forma rigorosamente matemática como

$$
S_{t}=S_{0}+\int_{0}^{t} \mu S_{u} d u+\int_{0}^{t} \sigma S_{u} d W_{u}
$$

ou seja, $\mathbf{S}$ é um processo estocástico que a para cada $t \geq 0$ satisfaz a equação integral acima P-q. s..

Pelo fato de $\mu$ e $\sigma$ serem constantes, a ede (5.12) satisfaz trivialmente as condições de existência e unicidade. Vamos ilustrar como a fórmula de Itô ajuda a encontrar a solução. Defina

$$
f(t, x) \doteq S_{0} \exp \left(\sigma x+\left(\mu-\frac{1}{2} \sigma^{2}\right) t\right)
$$

Temos

$$
f_{t}=S_{0}\left(\mu-\frac{1}{2} \sigma^{2}\right) f, \quad f_{x}=S_{0} \sigma f, \quad f_{x x}=S_{0} \sigma^{2} f
$$


e portanto, pela fórmula de Itô

$$
f\left(t, W_{t}\right)=S_{0}+\int_{0}^{t} \mu S_{0} \exp \left(\sigma W_{s}+\left(\mu-\frac{1}{2} \sigma^{2}\right) s\right) d s+\int_{0}^{t} \sigma S_{0} \exp \left(\sigma W_{s}+\left(\mu-\frac{1}{2} \sigma^{2}\right) s\right) d W_{s}
$$

isto de acordo com a notação diferencial* significa que

$$
S_{t}=S_{0} \exp \left(\sigma W_{t}+\left(\mu-\frac{1}{2} \sigma^{2}\right) t\right)
$$

é a única solução forte de (5.12). Tal processo é o protótipo do que definimos como um movimento Browniano geométrico.

Observamos que o modelo apresentado é considerado atualmente um modelo simples pela literatura de finanças e ilustra apenas uma possibilidade e que pode ser razoável ou não para problemas práticos. Para mais detalhes e aperfeiçoamentos deste modelo, recomendamos [9].

Exemplo 5.8. Vamos apresentar um exemplo simples de equação diferencial estocástica em $\mathbb{R}^{2}$. Sejam W um movimento Browniano e $Z_{t}=\left(X_{t}, Y_{t}\right)^{T}$ um processo estocástico bi-dimensional com $X_{t}=\cos \left(W_{t}\right)$ e $Y_{t}=\sin \left(W_{t}\right)$. Podemos interpretar $Z_{t}$ como a posição no instante $t \geq 0$ de um objeto que se movimenta no círculo unitário com ângulo dado por um movimento Browniano. Pela fórmula de Itô, temos que

$$
d X_{t}=-\sin \left(W_{t}\right) d W_{t}-\frac{1}{2} \cos \left(W_{t}\right) d t
$$

e

$$
d Y_{t}=\cos \left(W_{t}\right) d W_{t}-\frac{1}{2} \sin \left(W_{t}\right) d t
$$

Assim, a solução do problema

$$
d Z_{t}=\left(\begin{array}{cc}
0 & -1 \\
1 & 0
\end{array}\right) Z_{t} d W_{t}-\frac{1}{2} Z_{t} d t
$$

$\operatorname{com} Z_{0}=(1,0)^{T}$

\footnotetext{
* Veja a definição 4.47 na página 113.
} 
é dada por $\left(\cos \left(W_{t}\right), \sin \left(W_{t}\right)\right)^{T}$.

\subsection{O problema de Cauchy e a fórmula de Feynman-Kac}

É comum na literatura chamar a solução de uma equação diferencial estocástica de processo de difusão. O exemplo mais simples de uma equação diferencial parcial cuja solução pode ser expressa em termos de um processo de difusão é a equação do calor. Em termos físicos, considere uma barra infinita, termicamente isolada e seja $f(x)$ a temperatura na barra no instante $t=0$ e na posição $x$. Sendo $u(t, x)$ a temperatura da barra no instante $t \geq 0$ e posição $x \in \mathbb{R}, u$ satifaz

$$
\begin{cases}\partial_{t} u(t, x)-\frac{1}{2} \partial_{x x} u(t, x)=0, & t>0, x \in \mathbb{R} \\ u(0, x)=f(x), & x \in \mathbb{R}\end{cases}
$$

Agora, seja $p(t, x)$ a densidade da distribuição normal $N(0, t)$, isto é

$$
p(t, x)=\frac{1}{\sqrt{2 \pi t}} \exp \left(-\frac{x^{2}}{2 t}\right)
$$

De maneira formal e sem se preocupar com condições técnicas, diferenciando sob o sinal de integração não é difícil verificar que

$$
u(t, x)=\int_{-\infty}^{\infty} f(y) p(t, x-y) d y
$$

satisfaz a equação do calor para $t>0$.

De maneira mais precisa, suponha que $f: \mathbb{R} \rightarrow \mathbb{R}$ seja limitada, contínua e satisfaça

$$
\int_{-\infty}^{\infty} \exp \left(-a x^{2}\right)|f(x)| d x<\infty
$$

para algum $a>0$. Nessas condições, pode-se mostrar (veja [23], página 254) que $u$, dada por (5.13) está definida para $t \in\left(0, \frac{1}{2 a}\right)$ e $x \in \mathbb{R}$, possui derivada de todas as ordens, satisfaz a equação do calor e a condição inicial.

Agora seja W um movimento Browniano padrão unidimensional. Temos que $\mathbf{Y}=x+\mathbf{W}$ é um 
movimento Browniano que inicia em $x$ e além disso $Y_{t} \sim N(x, t)$. Portanto

$$
\mathbf{E}\left(f\left(x+W_{t}\right)\right)=\mathbf{E}\left(f\left(Y_{t}\right)\right)=\int_{-\infty}^{\infty} f(y) \frac{1}{\sqrt{2 \pi t}} \exp \left(-\frac{(y-x)^{2}}{2 t}\right) d y
$$

ou seja, $u(t, x)=\mathbf{E}\left(f\left(x+W_{t}\right)\right)$.

Sendo $u$ a solução da equação do calor, se fixarmos um valor terminal $T$ e definirmos $v(t, x) \doteq$ $u(T-t, x)$ para $t \in[0, T]$, então $v$ satisfaz a equação do calor reversa com a condição terminal $v(T, x)=f(x)$. Ou seja,

$$
\begin{cases}\partial_{t} v(t, x)+\frac{1}{2} \partial_{x x} v(t, x)=0, & t \in[0, T], x \in \mathbb{R} \\ v(T, x)=f(x), & x \in \mathbb{R}\end{cases}
$$

Temos portanto a seguinte representacao estocástica para $v$ :

$$
v(t, x)=u(T-t, x)=\mathbf{E}\left(f\left(x+W_{T-t}\right)\right) .
$$

Denotando por $W^{t, x}$ o processo definido em $[t, \infty)$ que começa no instante $t$ na posição $x$ e cujo os incrementos tem a mesma distribuição dos incrementos do movimento Browniano, temos que $W_{s}^{t, x}=x+W_{s-t}$, para $s \geq t$ e portanto

$$
v(t, x)=\mathbf{E}\left(f\left(W_{T}^{t, x}\right)\right)
$$

Note que relacionamos a solução de uma equação diferencial parcial com a solução de uma equação diferencial estocástica. De fato, $\mathbf{W}^{t, x}$ é solução de

$$
\left\{\begin{array}{l}
d X_{s}=d W_{s}, \quad s \geq t \\
X_{t}=x
\end{array}\right.
$$

Consideraremos nessa seção equações diferenciais parciais parabólicas, mais gerais do que a equação do calor e com condições terminais ao invés de inicais. O teorema de Feynman-Kac nós diz que a solução de tais equações podem ser representadas por funcionais das soluções de certas equações diferenciais estocásticas. 
Começaremos observando que para cada equação diferencial estócastica $d$-dimensional,

$$
d X_{t}^{(i)}=b_{i}\left(t, X_{t}\right) d t+\sum_{j=1}^{r} \sigma_{i j}\left(t, X_{t}\right) d W_{t}^{(j)}, \quad 1 \leq i \leq d .
$$

podemos associar uma matriz $d \times d$, definida como $a(t, x) \doteq \sigma(t, x) \sigma^{T}(t, x)$, cujas entradas são dadas por

$$
a_{i k}(t, x)=\sum_{j=1}^{r} \sigma_{i j}(t, x) \sigma_{k j}(t, x) ; \quad 1 \leq i, k \leq d .
$$

Chamamos esta matriz de matriz de difusão. Agora, para cada $t \geq 0$, vamos definir o operador diferencial $L_{t}$ que age sobre funções $f \in \mathcal{C}^{2}\left(\mathbb{R}^{d}\right)$ da seguinte maneira:

$$
\left(L_{t} f\right)(x) \doteq \frac{1}{2} \sum_{i=1}^{d} \sum_{k=1}^{d} a_{i k}(t, x) D_{i k} f(x)+\sum_{i=1}^{d} b_{i}(t, x) D_{i} f(x)
$$

Consideremos agora que para cada par $(t, x)$ existe uma única solução de (5.14) satisfazendo a condição inicial $X_{t}=x$. Denotaremos esse processo por $\mathbf{X}^{t, x}$ e observamos que este satisfaz a equação integral

$$
X_{s}^{t, x}=x+\int_{t}^{s} b\left(u, X_{u}^{t, x}\right) d u+\int_{t}^{s} \sigma\left(u, X_{u}^{t, x}\right) d W_{u}, \quad s \in[t, \infty)
$$

$\mathrm{P}-\mathrm{q} \cdot \mathrm{s}$.

Agora vamos lembrar uma notação. Seja $E$ um subconjunto aberto de $\mathbb{R}^{d}$. Se $f:[0, T) \times E \rightarrow \mathbb{R}$ for uma função contínua e se todas as suas derivadas de primeira e segunda ordem existirem e forem contínuas em $(0, T) \times E$ escreveremos $f \in \mathcal{C}^{1,2}((0, T) \times E)$. Quando $f \in \mathcal{C}^{1,2}((0, T) \times E)$ e suas derivadas parciais tiverem uma extensão contínua para $[0, T) \times E$, escreveremos $f \in \mathcal{C}^{1,2}([0, T) \times E)$. Vamos enunciar um lema que precisaremos usar. A demonstração pode ser encontrada em [23].

Lema 5.9. Suponha que as hipóteses do teorema 5.6 esteja satisfeitas e seja $\mathbf{X}$ a solução da equação diferencial estocástica. Então para $T>0$ fixo e $m \geq 1$, existe uma constante $C$ tal que

$$
\mathbf{E}\left(\max _{0 \leq s \leq t}\left\|X_{s}\right\|^{2 m}\right) \leq C e^{C t}\left(1+\|x\|^{2 m}\right)
$$

para todo $t \in[0, T]$. 
Teorema 5.10 (Fórmula de Feynman-Kac). Sejam $T>0$ fixo, $L>0, \lambda \geq 1$ constantes tais que

i) $f: \mathbb{R}^{d} \rightarrow \mathbb{R}$ é contínua e para todo $x \in \mathbb{R}^{d}$,

$$
|f(x)| \leq L\left(1+\|x\|^{2 \lambda}\right) \text { ou } f(x) \geq 0 .
$$

ii) $g:[0, T] \times \mathbb{R}^{d} \rightarrow \mathbb{R}$ é contínua e para todo $t \in[0, T]$ e $x \in \mathbb{R}^{d}$,

$$
|g(t, x)| \leq L\left(1+\|x\|^{2 \lambda}\right) \text { ou } g(t, x) \geq 0
$$

iii) $k:[0, T] \times \mathbb{R}^{d} \rightarrow[0, \infty)$ é contínua.

Além disso suponha que os coeficientes $b_{i}(t, x), \sigma_{i j}(t, x):[0, \infty) \times \mathbb{R}^{d} \rightarrow \mathbb{R}$ satifaçam as condições do teorema 5.6 de tal forma que exista e seja única a solução de (5.15). Temos que se v: $[0, T] \times \mathbb{R}^{d} \rightarrow \mathbb{R}^{d}$ é da classe $\mathcal{C}^{1,2}\left([0, T) \times \mathbb{R}^{d}\right)$ e é solução do problema de Cauchy

$$
\begin{cases}-\partial_{t} v+k v=L_{t} v+g, & t \in[0, T), x \in \mathbb{R}^{d} \\ v(T, x)=f(x), & x \in \mathbb{R}^{d}\end{cases}
$$

então a solução $v$, em $[0, T] \times \mathbb{R}^{d}$ pode ser escrita como

$$
\begin{aligned}
v(t, x) & =\mathbf{E}\left(f\left(X_{T}^{t, x}\right) \exp \left(-\int_{t}^{T} k\left(\theta, X_{\theta}^{t, x}\right) d \theta\right)\right) \\
& +\mathbf{E}\left(\int_{t}^{T} g\left(z, X_{z}^{t, x}\right) \exp \left(-\int_{t}^{z} k\left(\theta, X_{\theta}^{t, x}\right) d \theta\right) d z\right) .
\end{aligned}
$$

Além disso, não existe outra solução para o problema de Cauchy (5.18) que satisfaça a seguinte condição de crescimento polinomial:

$$
\max _{0 \leq t \leq T}|v(t, x)| \leq M\left(1+\|x\|^{2 \mu}\right)
$$

para todo $x \in \mathbb{R}^{d}$ e constantes $M>0$ e $\mu \geq 1$. 
Demonstração. Seja $F:[t, T] \times \mathbb{R}^{d}$, definida por

$$
F(s, y)=v(s, y) \exp \left(-\int_{t}^{s} k\left(\theta, X_{\theta}^{t, x}\right) d \theta\right)
$$

Pela fórmula de Itô, temos que

$$
\begin{aligned}
F\left(s, X_{s}^{t, x}\right)-F\left(t, X_{t}^{t, x}\right) & =\int_{t}^{s} \frac{\partial F}{\partial s}\left(z, X_{z}^{t, x}\right) d z+\sum_{i=1}^{d} \int_{t}^{s} D_{i} F\left(z, X_{z}^{t, x}\right) d X_{z}^{(i)} \\
& +\frac{1}{2} \sum_{i, j=1}^{d} \int_{t}^{s} D_{i j} F\left(z, X_{z}^{t, x}\right) d \llbracket \mathbf{X}^{(\mathbf{i})}, \mathbf{X}^{(\mathbf{j})} \rrbracket_{z}
\end{aligned}
$$

Agora faremos uma série de observações.

- $F\left(t, X_{t}^{t, x}\right)=v\left(t, X_{t}^{t, x}\right) \exp \left(-\int_{t}^{t} k\left(\theta, X_{\theta}^{t, x}\right) d \theta\right)=v(t, x)$.

$$
\begin{aligned}
\frac{\partial F}{\partial s}\left(z, X_{z}^{t, x}\right) & =\partial_{t} v\left(z, X_{z}^{t, x}\right) \exp \left(-\int_{t}^{z} k\left(\theta, X_{\theta}^{t, x}\right) d \theta\right) \\
& -v\left(z, X_{z}^{t, x}\right) \exp \left(-\int_{t}^{z} k\left(\theta, X_{\theta}^{t, x}\right) d \theta\right) k\left(z, X_{z}^{t, x}\right) \\
& =\exp \left(-\int_{t}^{z} k\left(\theta, X_{\theta}^{t, x}\right) d \theta\right)\left[\partial_{t} v\left(z, X_{z}^{t, x}\right)-v\left(z, X_{z}^{t, x}\right) k\left(z, X_{z}^{t, x}\right)\right]
\end{aligned}
$$

- De (5.14), temos

$$
d X_{t}^{(i)}=b_{i}\left(t, X_{t}\right) d t+\sum_{j=1}^{r} \sigma_{i j}\left(t, X_{t}\right) d W_{t}^{(j)}, \quad 1 \leq i \leq d
$$


e portanto pela bilinearidade da integral estocástica,

$$
\begin{aligned}
\sum_{i=1}^{d} \int_{t}^{s} D_{i} F\left(z, X_{z}^{t, x}\right) d X_{z}^{(i)} & =\sum_{i=1}^{d} \int_{t}^{s} D_{i} F\left(z, X_{z}^{t, x}\right) b_{i}\left(z, X_{z}^{t, x}\right) d z \\
& +\sum_{i=1}^{d} \sum_{j=1}^{r} \int_{t}^{s} D_{i} F\left(z, X_{z}^{t, x}\right) \sigma_{i j}\left(z, X_{z}^{t, x}\right) d W_{z}^{(j)} \\
& =\sum_{i=1}^{d} \int_{t}^{s} D_{i} v\left(z, X_{z}^{t, x}\right) \exp \left(-\int_{t}^{z} k\left(\theta, X_{\theta}^{t, x}\right) d \theta\right) b_{i}\left(z, X_{z}^{t, x}\right) d z \\
& +\sum_{i=1}^{d} \sum_{j=1}^{r} \int_{t}^{s} D_{i} F\left(z, X_{z}^{t, x}\right) \sigma_{i j}\left(z, X_{z}^{t, x}\right) d W_{z}^{(j)} .
\end{aligned}
$$

- De acordo com notação diferencial para produtos (veja (4.30)) temos que $d \llbracket \mathbf{X}^{(\mathbf{i})}, \mathbf{X}^{(\mathbf{j})} \rrbracket_{z}=$ $d X_{z}^{i} d X_{z}^{j}$ e portanto usando (5.14) e a tabela de cálculo (veja a proposição 4.53 na página 120) ficamos com

$$
\begin{aligned}
d \llbracket \mathbf{X}^{(\mathbf{i})}, \mathbf{X}^{(\mathbf{j})} \|_{z} & =d X_{z}^{i} d X_{z}^{j} \\
& =\left(b_{i}\left(z, X_{z}^{t, x}\right) d z+\sum_{l=1}^{r} \sigma_{i l}\left(z, X_{z}^{t, x}\right) d W_{z}^{(l)}\right)\left(b_{j}\left(z, X_{z}^{t, x}\right) d z+\sum_{l=1}^{r} \sigma_{j l}\left(z, X_{z}^{t, x}\right) d W_{z}^{(l)}\right) \\
& =\sum_{l=1}^{r} \sigma_{i l}\left(z, X_{z}^{t, x}\right) \sigma_{j l}\left(z, X_{z}^{t, x}\right) d z \\
& =a_{i j}\left(z, X_{z}^{t, x}\right) .
\end{aligned}
$$

- $D_{i j} F\left(z, X_{z}^{t, x}\right)=D_{i j} v\left(t, X_{z}^{t, x}\right) \exp \left(-\int_{t}^{z} k\left(\theta, X_{\theta}^{t, x}\right) d \theta\right)$. 
Utilizando essas observações temos que

$$
\begin{aligned}
F\left(s, X_{s}^{t, x}\right)-v(t, x) & =\int_{t}^{s} \exp \left(-\int_{t}^{z} k\left(\theta, X_{\theta}^{t, x}\right) d \theta\right)\left[\partial_{t} v\left(z, X_{z}^{t, x}\right)-k\left(z, X_{z}^{t, x}\right) v\left(z, X_{z}^{t, x}\right)\right] d z \\
& +\int_{t}^{s} \exp \left(-\int_{t}^{z} k\left(\theta, X_{\theta}^{t, x}\right) d \theta\right) \sum_{i=1}^{d} b_{i}\left(z, X_{z}^{t, x}\right) D_{i} v\left(z, X_{z}^{t, x}\right) d z \\
& +\sum_{i=1}^{d} \sum_{j=1}^{r} \int_{t}^{s} D_{i} F\left(z, X_{z}^{t, x}\right) \sigma_{i j}\left(z, X_{z}^{t, x}\right) d W_{z}^{(j)} \\
& +\int_{t}^{s} \exp \left(-\int_{t}^{z} k\left(\theta, X_{\theta}^{t, x}\right) d \theta\right) \frac{1}{2} \sum_{i, j=1}^{d} a_{i j}\left(z, X_{z}^{t, x}\right) D_{i j} v\left(z, X_{z}^{t, x}\right) d z \\
& =\int_{t}^{z} \exp \left(-\int_{t}^{z} k\left(\theta, X_{\theta}^{t, x}\right) d \theta\right)\left[v\left(z, X_{z}^{t, x}\right)-k\left(z, X_{z}^{t, x}\right) v\left(z, X_{z}^{t, x}\right)+L_{t} v\left(z, X_{z}^{t, x}\right)\right] d z \\
& +\sum_{i=1}^{d} \sum_{j=1}^{r} \int_{t}^{s} D_{i} F\left(z, X_{z}^{t, x}\right) \sigma_{i j}\left(z, X_{z}^{t, x}\right) d W_{z}^{(j)} .
\end{aligned}
$$

Agora, do fato de que $v$ é solução do problema de Cauchy e a definição do operador $L_{t}$, temos que (5.20) pode ser escrito como

$$
\begin{aligned}
F\left(s, X_{s}^{t, x}\right)-v(t, x) & =-\int_{t}^{s} \exp \left(-\int_{t}^{z} k\left(\theta, X_{\theta}^{t, x}\right) d \theta\right) g\left(z, X_{z}^{t, x}\right) d z \\
& +\sum_{i=1}^{d} \sum_{j=1}^{r} \int_{t}^{s} D_{i} F\left(z, X_{z}^{t, x}\right) \sigma_{i j}\left(z, X_{z}^{t, x}\right) d W_{z}^{(j)}
\end{aligned}
$$

Note que ao trocar $s$ por $T$ e tomar o valor esperado dos dois lados da igualdade acima, o resultado estaria provado se valesse que

$$
\mathbf{E}\left(\sum_{i=1}^{d} \sum_{j=1}^{r} \int_{t}^{T} D_{i} F\left(z, X_{z}^{t, x}\right) \sigma_{i j}\left(z, X_{z}^{t, x}\right) d W_{z}^{(j)}\right)=0,
$$

mas isso não é imediatamente evidente. A estratégia será usar tempos de parada. Pela definição de 
$F$, temos que para todo $i \in\{1, \ldots, d\}$ e $j \in\{1, \ldots, r\}$,

$$
\int_{t}^{s} D_{i} F\left(z, X_{z}^{t, x}\right) \sigma_{i j}\left(z, X_{z}^{t, x}\right) d W_{z}^{(j)}=\int_{t}^{s} D_{i} v\left(z, X_{z}^{t, x}\right) \exp \left(-\int_{t}^{z} k\left(\theta, X_{\theta}^{t, x}\right) d \theta\right) \sigma_{i j}\left(z, X_{z}^{t, x}\right) d W_{z}^{(j)} .
$$

Agora, para simplificar a notação vamos denotar $X^{t, x}=X$ e definir o processo $\left\{M_{s}: s \in[t, T]\right\}$ por

$$
M_{s}=\int_{t}^{s} D_{i} v\left(z, X_{z}\right) \exp \left(-\int_{t}^{z} k\left(\theta, X_{\theta}\right) d \theta\right) \sigma_{i j}\left(z, X_{z}\right) d W_{z}^{(j)}
$$

ou, de forma equivalente

$$
M_{s}=\int_{0}^{s} \mathbb{1}_{\{z \geq t\}} D_{i} v\left(z, X_{z}\right) \exp \left(-\int_{t}^{z} k\left(\theta, X_{\theta}\right) d \theta\right) \sigma_{i j}\left(z, X_{z}\right) d W_{z}^{(j)}
$$

Seja $\tau^{n} \doteq \inf \left\{s \geq t:\left|X_{s}\right| \geq n\right\}$, e para cada $n \geq 1$, considere o processo $\left\{\left(M^{\tau_{n}}\right)_{s}\right\}_{s \in[t, T]}$. Dessa forma, temos que o valor de $\mathbf{M}^{\tau_{n}}$ no instante $s$ é dado por

$$
\begin{aligned}
\left(M^{\tau_{n}}\right)_{s} & =\int_{0}^{s \wedge \tau_{n}} \mathbb{1}_{\{z \geq t\}} D_{i} v\left(z, X_{z}\right) \exp \left(-\int_{t}^{z} k\left(\theta, X_{\theta}\right) d \theta\right) \sigma_{i j}\left(z, X_{z}\right) d W_{z}^{(j)} \\
& =\int_{0}^{s} \mathbb{1}_{\left\{z \leq \tau_{n}\right\}} \mathbb{1}_{\{z \geq t\}} D_{i} v\left(z, X_{z}^{\tau_{n}}\right) \exp \left(-\int_{t}^{z} k\left(\theta, X_{\theta}^{\tau_{n}}\right) d \theta\right) \sigma_{i j}\left(z, X_{z}^{\tau_{n}}\right) d W_{z}^{(j)} .
\end{aligned}
$$

Agora, defina

$$
g(z, \omega) \doteq \mathbb{1}_{\left\{z \leq \tau_{n}(\omega)\right\}} \mathbb{1}_{\{z \geq t\}} D_{i} v\left(z, X_{z}^{\tau_{n}(\omega)}(\omega)\right) \sigma_{i j}\left(z, X_{z}^{\tau_{n}(\omega)}(\omega)\right) \exp \left(-\int_{t}^{z} k\left(u, X_{u}^{\tau_{n}(\omega)}(\omega)\right) d u\right)
$$

Note que por $D_{i} v, \sigma$ e exp serem funções contínuas, $g$ também é. Além disso, como $\mathbf{X}$ é um processo adaptado (pois é por hipótese a solução forte de uma ede), $g$ também é e portanto* $g$ é progressivamente mensurável. Agora, como $\left|X^{\tau_{n}}\right|$ é uniformemente limitado (por $n$ ), $v_{x}$ and $\sigma$ são limitadas em conjuntos compactos e $\mid \exp \left(-\int_{t}^{z} k\left(u, X_{u}^{\tau_{n}}\right) d u \mid \leq 1\right.$ (pois $k \geq 0$ ) temos que $g$ é limitado

* Veja a proposição 2.22 na página 14. 
e portanto (para todo $\omega \in \Omega$ )

$$
\int_{0}^{s}|g(z, \omega)|^{2} d z<\infty, \quad \forall s \in[t, T]
$$

Isto quer dizer que $g \in \Lambda^{2}\left(\mathbf{W}^{(j)}\right)$, e assim* para cada $n \geq 1,\left\{\left(M^{\tau_{n}}\right)_{s}\right\}_{s \in[t, T]}$ é um martingal de quadrado integrável. Dessa forma segue que

$$
E\left[M_{T}^{\tau_{n}}\right]=E\left[M_{t}^{\tau_{n}}\right]=0
$$

(Para justificar essa última igualdade, perceba que $\tau_{n} \wedge t=t$ e portanto a integral que define $\mathbf{M}^{\tau_{n}}$ se anula.)

Como as contas acima valem para todo $i$ e $j$, segue, pela linearidade da esperança que

$$
\mathbf{E}\left(\sum_{i=1}^{d} \sum_{j=1}^{r} \int_{t}^{T \wedge \tau_{n}} D_{i} F\left(z, X_{z}^{t, x}\right) \sigma_{i j}\left(z, X_{z}^{t, x}\right) d W_{z}^{(j)}\right)=0
$$

Agora fazendo $s=T \wedge \tau_{n}$ em (5.22) e tomando o valor esperado, ficamos com

$$
\mathbf{E}\left(F\left(T \wedge \tau_{n}, X_{T \wedge \tau_{n}}^{t, x}\right)\right)-v(t, x)=-\mathbf{E}\left(\int_{t}^{T \wedge \tau_{n}} g\left(z, X_{z}^{t, x}\right) \exp \left(-\int_{t}^{z} k\left(\theta, X_{\theta}^{t, x}\right) d \theta\right) d z\right)
$$

ou seja,

$$
\begin{aligned}
v(t, x) & =\mathbf{E}\left(v\left(T \wedge \tau_{n}, X_{T \wedge \tau_{n}}^{t, x}\right) \exp \left(-\int_{t}^{T \wedge \tau_{n}} k\left(\theta, X_{\theta}^{t, x}\right) d \theta\right)\right) \\
& +\mathbf{E}\left(\int_{t}^{T \wedge \tau_{n}} g\left(z, X_{z}^{t, x}\right) \exp \left(-\int_{t}^{z} k\left(\theta, X_{\theta}^{t, x}\right) d \theta\right) d z\right)
\end{aligned}
$$

Agora, o trabalho será fazer $n \rightarrow \infty$ e usar o teorema da convergência dominada para justificar a troca do sinal de limite com o da esperança. Para isso, note inicialmente que primeira parcela

\footnotetext{
* Veja a observação feita após a definição 4.29 na página 89.
} 
de (5.23) pode ser decomposta como a soma de

$$
\mathbf{E}\left(f\left(X_{T}^{t, x}\right) \exp \left(-\int_{t}^{T} k\left(\theta, X_{\theta}^{t, x}\right) d \theta\right) \mathbb{1}_{\left\{\tau_{n}>T\right\}}\right)
$$

$\mathrm{e}$

$$
\mathbf{E}\left(v\left(\tau_{n}, X_{\tau^{n}}^{t, x}\right) \exp \left(-\int_{t}^{\tau_{n}} k\left(\theta, X_{\theta}^{t, x}\right) d \theta\right) \mathbb{1}_{\left\{\tau_{n} \leq T\right\}}\right) .
$$

Note que por (5.16), a expressão (5.24) é limitada em valor absoluto por $L \mathbf{E}\left(1+\left\|X_{T}\right\|^{2 \lambda}\right)$. Agora, pelo lema 5.9, $\mathbf{E}\left(\left\|X_{T}\right\|^{2 \lambda}\right) \leq C e^{C(T-t)}\left(1+\|x\|^{2 \lambda}\right)<\infty$. Assim, pelo teorema da convergência dominada, temos que o limite de (5.24) para $n \rightarrow \infty$, é

$$
\mathbf{E}\left(f\left(X_{T}^{t, x}\right) \exp \left(-\int_{t}^{T} k\left(\theta, X_{\theta}^{t, x}\right) d \theta\right)\right)
$$

Agora temos por (5.19), que a expressão (5.25) é limitada em valor absoluto por $M\left(1+n^{2 \mu}\right) \mathrm{P}\left(\tau_{n} \leq T\right)$. Usando a desigualdade de Chebychev* e o lema 5.9, temos que

$$
\begin{aligned}
M\left(1+n^{2 \mu}\right) \mathrm{P}\left(\tau_{n} \leq T\right) & =M\left(1+n^{2 \mu}\right) \mathrm{P}\left(\sup _{t \leq s \leq T}\left\|X_{s}^{t, x}\right\| \geq n\right) \\
& \leq M\left(1+n^{2 \mu}\right) n^{-2 m} \mathbf{E}\left(\sup _{t \leq s \leq T}\left\|X_{s}^{t, x}\right\|^{2 m}\right) \\
& \leq M\left(1+n^{2 \mu}\right) n^{-2 m} C e^{C(T-t)}\left(1+\|x\|^{2^{m}}\right) .
\end{aligned}
$$

Assim, se tomarmos $m>\mu$,

$$
\lim _{n \rightarrow \infty} \mathbf{E}\left(v\left(\tau_{n}, X_{\tau^{n}}^{t, x}\right) \exp \left(-\int_{t}^{\tau_{n}} k\left(\theta, X_{\theta}^{t, x}\right) d \theta\right) \mathbb{1}_{\left\{\tau_{n} \leq T\right\}}\right)=0 .
$$

Finalmente, tratamos a última parcela de (5.23) de maneira similar. De fato, por (5.17) e o

\footnotetext{
* Veja o corolário 2.32 na página 19.
} 
lema 5.9 podemos usar o teorema da convergência dominada, (ou também convergência monótona caso $g(t, x) \geq 0)$ e portanto,

$$
\begin{aligned}
\lim _{n \rightarrow \infty} \mathbf{E}\left(\int_{t}^{T \wedge \tau_{n}} g\left(z, X_{z}^{t, x}\right) \exp \left(-\int_{t}^{z} k\left(\theta, X_{\theta}^{t, x}\right) d \theta\right) d z\right) & = \\
& \mathbf{E}\left(\int_{t}^{T} g\left(z, X_{z}^{t, x}\right) \exp \left(-\int_{t}^{z} k\left(\theta, X_{\theta}^{t, x}\right) d \theta\right) d z\right) .
\end{aligned}
$$

Finalmente, de (5.26), (5.27), (5.28) e (5.23) resulta a fórmula de Feynman-Kac.

Assim, se pudermos mostrar que o problema de Cauchy (5.18) possui uma solução que satisfaça a condição de crescimento (5.19), então tal solução é única e possui uma representação probabilística dada pela fórmula de Feynman-Kac.

Exemplo 5.11. Neste exemplo, vamos usar a representação probabilística da solução de um problema de Cauchy parabólico para determinar sua solução explícita.

$$
\begin{cases}-\partial_{t} v+k v=\frac{1}{2} \sigma^{2} x^{2} \partial_{x x} v+\mu x \partial_{x} v, & t \in[0, T), x \in \mathbb{R} \\ v(T, x)=x^{2}, & x \in \mathbb{R}\end{cases}
$$

Neste exemplo, $\sigma, \mu$ e $k$ são constantes positivas. Para começar, defina o operador

$$
\left(L_{t} v\right)(x)=\frac{1}{2}(\sigma x)(\sigma x) \partial_{x x} v(x)+(\mu x) \partial_{x} v .
$$

Associado a esse operador, temos a equação diferencial estócastica

$$
d X_{t}=\mu X_{t} d t+\sigma X_{t} d W_{t}
$$

cuja a solução, conforme vimos no exemplo 5.7, é dada por

$$
X_{t}=X_{0} \exp \left[\sigma W_{t}+\left(\mu-\frac{\sigma^{2}}{2}\right) t\right] .
$$


Assim, a solução para o problema de valor inicial estócastico

$$
\left\{\begin{array}{l}
d X_{s}=\mu X_{s} d s+\sigma X_{s} d W_{s}, \quad s \geq t \\
X_{t}=x
\end{array}\right.
$$

é dada por

$$
X_{s}=X_{t} \exp \left[\sigma\left(W_{s}-W_{t}\right)+\left(\mu-\frac{\sigma^{2}}{2}\right)(s-t)\right]
$$

e será denotada por $X_{s}^{t, x}$. De acordo com a fórmula de Feynman-Kac, temos que se existir uma solução para o problema (5.29) que satisfaça a condição crescimento polinomial (5.19), então esta solução é dada por

$$
\begin{aligned}
v(t, x) & =\mathbf{E}\left(\left(X_{T}^{t, x}\right)^{2} \exp [-k(T-t)]\right) \\
& =\exp [-k(T-t)] \mathbf{E}\left(\left(X_{T}^{t, x}\right)^{2}\right) .
\end{aligned}
$$

Neste caso, podemos calcular a fórmula explícita da solução, pois tendo em vista (5.31)

$$
\mathbf{E}\left(\left(X_{T}^{t, x}\right)^{2}\right)=x^{2} \exp \left[\left(2 \mu-\sigma^{2}\right)(T-t)\right] \mathbf{E}\left(\exp \left[2 \sigma\left(W_{T}-W_{t}\right)\right]\right)
$$

Agora, pela definição de movimento Browniano, temos que $W_{T}-W_{t} \sim N(0, T-t)$ e pela discussão sobre funções geradoras de momentos no apêndice $\mathrm{D}$, segue que

$$
\mathbf{E}\left(\exp \left[2 \sigma\left(W_{T}-W_{t}\right)\right]\right)=\exp \left[2 \sigma^{2}(T-t)\right]
$$

Assim, substituindo (5.33) e (5.34) em (5.32), resulta

$$
\begin{aligned}
v(t, x) & =\exp [-k(T-t)] x^{2} \exp \left[\left(2 \mu-\sigma^{2}\right)(T-t)\right] \exp \left[2 \sigma^{2}(T-t)\right] \\
& =x^{2} \exp \left[\left(2 \mu-\sigma^{2}-k\right)(T-t)\right]
\end{aligned}
$$

Em alguns modelos de apreçamento de derivativos em finanças, o preço é dado por uma esperança condicional. Em algumas situações é mais simples resolver (até mesmo numericamente) uma equação diferencial parcial do que calcular uma esperança. Nessa situação, usa-se a fórmula de Feynman- 
Kac. Isso deve ser feito com cuidado pois a fórmula de Feynman-Kac que desenvolvemos nessa seção vai na direção oposta: Dado um problema de Cauchy parabólico, determina-se uma representação estocástica para a solução. Em algumas aplicações em finanças começamos com a representação estocástica para o preço e procura-se uma equação diferencial parcial que este satifaz. Este tipo de recíproca da fórmula de Feynman-Kac depende de algumas condições de integrabilidade da esperança e propriedades de Markov dos processos envolvidos. O leitor pode obter mais informações sobre isso em por exemplo [18] ou [20]. 


\section{Apêndice A}

\section{Teorema da classe monótona}

To isolate mathematics from the practical demands of the sciences is to invite the sterility of a cow shut away from the bulls.

Pafnuty Chebyshev

Definição A.1. Dizemos que uma familia $\mathcal{L}$ de subconjuntos de $\Omega$ é um $\lambda$-sistema em $\Omega$ quando estão satisfeitas as seguintes condições:

i) $\emptyset \in \mathcal{L}$,

ii) $S e A \in \mathcal{L}$ então $A^{c} \in \mathcal{L}$,

iii) $S e\left\{A_{n}\right\} \subseteq \mathcal{L}$ é uma sequência disjunta, então $\cup_{n} A_{n} \in \mathcal{L}$.

Dizemos também que uma família de subconjuntos de $\Omega$ é um $\pi$-sistema em $\Omega$ quando esta for fechada por interseções finitas.

O próximo teorema é conhecido como o teorema da classe monótona para conjuntos. Uma demonstração pode ser encontrada por exemplo em [16].

Teorema A.2. Seja $\mathcal{I}$ um $\pi$-sistema em $\Omega$ e $\mathcal{L}$ um $\lambda$-sistema em $\Omega$. Se $\mathcal{I} \subseteq \mathcal{L}$ então $\sigma(\mathcal{I}) \subseteq \mathcal{L}$. 
Outro teorema muito útil é o teorema da extensão. Para uma demonstração veja [16].

Teorema A.3. Seja $\mathcal{C}$ um familia de funções não negativas e $\mathscr{F}$-mensuráveis definidas em $\Omega$ satisfazendo as seguintes propriedades:

i) $\mathcal{C}$ contém a função constante 1 .

ii) Se $f, g \in \mathcal{C}$ são limitadas e $f \leq g$ então $f-g \in \mathcal{C}$.

iii) Se para todo $n \geq 1, f_{n} \in \mathcal{C}$ e $\alpha_{n} \geq 0$, então $f=\sum_{n} \alpha_{n} f_{n} \in \mathcal{C}$.

Se $\mathbb{1}_{[A]} \in \mathcal{C}$, para cada conjunto A em um $\pi$-sistema $\mathcal{I}$ que gera a $\sigma$-àlgebra $\mathscr{F}$, então $\mathcal{C}$ contém todas as funções não-negativas e mensuráveis definidas em $\Omega$.

Dizemos que uma família $\mathcal{C}$ satisfazendo as condições i,ii e iii acima é um $\lambda$-cone em $\Omega$. 


\title{
Apêndice B
}

\section{Convergência de variavéis aleatórias}

\author{
As far as the laws of mathematics refer to reality, \\ they are not certain, and as far as they are \\ certain, they do not refer to reality.
}

Albert Einstein

Definição B.1. Sejam $X, X_{n}, n \geq 1$ variavéis aleatórias definidas no espaço de probabilidade $(\Omega, \mathscr{F}, \mathrm{P})$ e $1 \leq p<\infty$. Dizemos que

i) $X_{n}$ converge para $X$ em $\mathrm{L}^{p}$ e escrevemos $X_{n} \stackrel{\mathrm{L}^{p}}{\rightarrow} X$, quando

$$
\left\|X_{n}-X\right\|_{p}^{p}=\mathbf{E}\left(\left|X_{n}-X\right|^{p}\right) \longrightarrow 0, \text { para } n \uparrow \infty .
$$

ii) $X_{n}$ converge $\mathrm{P}$-quase sempre (P-q.s.) para $X$ e escrevemos $X_{n} \stackrel{\text { q.s }}{\longrightarrow} X$, quando

$$
X_{n}(\omega) \longrightarrow X(\omega) \text {, para todo } \omega \text { em um complementar de um conjunto P-nulo. }
$$

Nesse caso dizemos também que a convergência é quase certa.

iii) $X_{n}$ converge em probabilidade para $X$ e escrevemos $X_{n} \stackrel{\mathrm{P}}{\rightarrow} X$, quando

$$
\mathrm{P}\left(\left|X_{n}-X\right|>\varepsilon\right) \longrightarrow 0 \text {, para } n \uparrow \infty \text { e qualquer } \varepsilon>0 \text {. }
$$


Lembramos o leitor o fato de que pela medida de probabilida ser finita, $X_{n} \stackrel{\mathrm{L}^{p}}{\longrightarrow} X$ implica que $X_{n} \stackrel{\mathrm{L}^{r}}{\rightarrow} X$ para todo $1 \leq r \leq p$. O próximo resultado também usa esse fato. A demonstração pode ser encontrada em [1].

\section{Proposição B.2.}

i) Convergência P-q.s. implica na convergência em probabilidade.

ii) Convergência em $\mathrm{L}^{1}$ implica na convergência em probabilidade.

iii) Convergência em probabilidade implica na convergência P-q. s. de uma subsequência.

Desse resultado e da última observação, resulta facilmente que convergência em $\mathrm{L}^{p}$ implica na convergência quase certa de uma subsequência.

O próximo resultado será útil para algumas demonstrações usadas neste trabalho.

Proposição B.3. Seja $A_{k} \in \mathscr{F}, k \geq 1$ e $\Omega=\bigcup_{k} A_{k}$. Se $X_{n} \stackrel{\mathrm{P}}{\rightarrow} X$ em cada conjunto $A_{k}$, então $X_{n} \stackrel{\mathrm{P}}{\rightarrow} X$ em $\Omega$.

Demonstração. Podemos, sem perda de generalidade, supor que os conjuntos $A_{k}$ são dois a dois disjuntos. Agora, sejam $\varepsilon, \delta>0$ arbitrários e defina $B_{m} \doteq \bigcup_{k>m} A_{k}$. Seja $r$ suficientemente grande de tal forma que $\mathrm{P}\left(B_{r}\right)<\delta$. Então pela definição elementares de medida, temos para todo $n \geq 1$,

$$
\mathrm{P}\left(\left[\mid X_{n}-X>\varepsilon\right] \cap \Omega\right) \leq \sum_{k \leq r} \mathrm{P}\left(\left[\mid X_{n}-X>\varepsilon\right] \cap A_{k}\right)+\mathrm{P}\left(B_{r}\right)
$$

Assim,

$$
\limsup _{n} \mathrm{P}\left(\left|X_{n}-X\right|>\varepsilon\right) \leq P\left(B_{r}\right)<\delta .
$$

Da arbitrariedade de $\delta$, temos que $\lim \sup _{n} \mathrm{P}\left(\left|X_{n}-X\right|>\varepsilon\right)=0$ e como cada termo $\mathrm{P}\left(\left|X_{n}-X\right|>\varepsilon\right)$ é não negativo, resulta que para $n \uparrow \infty, \mathrm{P}\left(\left|X_{n}-X\right|>\varepsilon\right) \longrightarrow 0$, como queríamos.

Definição B.4. Dizemos que uma familia de variavéis aleatórias reais $\left\{X_{t}: t \in \Theta\right\}$ definidas em um espaço de probabilidade $(\Omega, \mathscr{F}, \mathrm{P})$ é uniformemente integrável quando

$$
\sup _{t \in \Theta} \int_{\left|X_{t}\right|>\lambda}\left|X_{t}\right| d \mathrm{P} \downarrow 0, \text { quando } \lambda \rightarrow \infty .
$$


Lema B.5. Seja $\left\{X_{\alpha}\right\}_{\alpha \in A}$ uma família de variavéis aleatórias uniformemente integrável. Então esta familía é limitada em $\mathrm{L}^{1}$.

Demonstração. De fato, para todo $\varepsilon>0$, existe um $\lambda>0$ tal que

$$
\mathbf{E}\left(\left|X_{\alpha}\right|\right)=\int_{\left|X_{\alpha}\right| \leq \lambda}\left|X_{\alpha}\right| d \mathrm{P}+\int_{\left|X_{\alpha}\right|>\lambda}\left|X_{\alpha}\right| d \mathrm{P} \leq \lambda+\varepsilon
$$

para todo $\alpha \in A$.

Um resultado importante relacionando conceitos de convergência com integrabilidade uniforme é dado no próximo teorema. A demonstração pode ser encontrada por exemplo em [27].

Teorema B.6. Sejam $X \in \mathrm{L}^{1}$ e $\left\{X_{n}\right\}_{n \in \mathbb{N}}$ uma sequência em $\mathrm{L}^{1}$. Então $\lim _{n \rightarrow \infty}\left\|X_{n}-X\right\|_{1}=0$, se e somente se, $\left\{X_{n}\right\}_{n \in \mathbb{N}}$ for uniformemente integrável e $\lim _{n \rightarrow \infty} X_{n}=X$ em probabilidade.

Corolário B.7. Seja $\left\{X_{n}\right\}_{n \geq 1}$ uma sequência em $\mathrm{L}^{1}$ e suponha que $X_{n} \stackrel{\text { q.s }}{\longrightarrow} X$. Então são equivalentes:

i) $X \in \mathrm{L}^{1}$ e $X_{n} \stackrel{\mathrm{L}^{1}}{\rightarrow} X$

ii) A família $\left\{X_{n}\right\}_{n \geq 1}$ é uniformemente integrável.

Demonstração. $\mathbf{i} \Rightarrow$ ii: Segue imediatamente do resultado anterior.

ii $\Rightarrow$ i: De fato, como a sequência $\left\{X_{n}\right\}_{n \geq 1}$ é uniformemente limitada, pelo Lema B.5 ela é também limitada em $\mathrm{L}^{1}$. Assim, da convergência quase certa e do lema de Fatou resulta

$$
\|X\|_{1}=\mathbf{E}(|X|)=\mathbf{E}\left(\liminf _{n}\left|X_{n}\right|\right) \leq \liminf _{n} \mathbf{E}\left(\left|X_{n}\right|\right)<\infty
$$

como queríamos.

Uma condição suficiente para integrabilidade uniforme é dada no próximo teorema cuja demonstração também pode ser encontrada em [27].

Teorema B.8. Seja $\left\{X_{t}: t \in \Theta\right\}$ uma familía de variáveis aleatórias reais limitadas em $\mathrm{L}^{p_{0}}$ para algum $p_{0} \in(0, \infty)$, isto é $\sup _{t \in \Theta} \mathbf{E}\left(\left|X_{t}\right|^{p_{0}}\right)<\infty$. Então $\left\{\left|X_{t}\right|^{p}: t \in \Theta\right\}$ é uniformemente integrável para cada $p \in\left(0, p_{0}\right)$. 
Vamos enunciar agora mais algumas propriedades que famílias de variaveis aleatórias uniformemente integráveis satisfazem (para demonstração, consulte [27]).

Proposição B.9. Seja $\left\{X_{\alpha}\right\}_{\alpha \in A}$ uma familia uniformemente integrável de variaveis aleatórias reais estendidas. Então

i) Se $\left\{c_{\alpha}: \alpha \in A\right\}$ é uma família de números reais limitados então $\left\{c_{\alpha} X_{\alpha}\right\}_{\alpha \in A}$ é uniformemente integrável.

ii) Se $Y \in E_{\infty}$ então $\left\{X_{\alpha} Y\right\}_{\alpha \in A}$ é uniformemente integrável.

iii) Se $\left\{Y_{\alpha}\right\}_{\alpha \in A}$ é outra família uniformemente integrável de variaveis aleatórias então $\left\{X_{\alpha}+Y_{\alpha}\right\}_{\alpha \in A}$ também é uniformemente integrável.

iv) Qualquer sub-família de $\left\{X_{\alpha}\right\}_{\alpha \in A}$ também é uniformemente integrável.

v) $\left\{X_{\alpha}\right\}_{\alpha \in A}$ é uniformemente integrável se, e somente se, $\left\{\left|X_{\alpha}\right|\right\}_{\alpha \in A}$ for.

vi) Seja $Y_{\alpha}=X_{\alpha}$ ou $-X_{\alpha}$ para cada $\alpha \in A$. Então $\left\{X_{\alpha}\right\}_{\alpha \in A}$ é uniformemente integrável se, $e$ somente se, $\left\{Y_{\alpha}\right\}_{\alpha \in A}$ for.

vii) Se $\left|Y_{\alpha}\right| \leq\left|X_{\alpha}\right|$ para todo $\alpha \in A$ então $\left\{Y_{\alpha}\right\}_{\alpha \in A}$ também é uniformemente integrável. Em particular, se $X$ for uma variável aleatória integrável e $\left|Y_{\alpha}\right| \leq X$ para todo $\alpha \in A$, então $\left\{Y_{\alpha}\right\}_{\alpha \in A}$ é uniformemente integrável.

viii) Toda familia finita $\left\{Y_{n}: n=1, \ldots, N\right\}$ de variavéis aleatórias estendidas e integráveis é uniformemente integrável. 


\section{Apêndice $\mathrm{C}$}

\section{Esperança condicional}

It is not certain that everything is uncertain.

Blaise Pascal

No que se segue, $\operatorname{sejam}(\Omega, \mathscr{F}, \mu)$ um espaço de medida e $f$ uma função não negativa e $\mathscr{F}$ mensurável.

Proposição C.1. Nas condições acima, seja $\nu: \mathscr{F} \rightarrow \mathbb{R}_{+}$dada por $\nu(A)=\int_{A} f d \mu$. Temos que $\nu$ é uma medida em $(\Omega, \mathscr{F})$.

Demonstração. Em primeiro lugar, $\nu(A) \in[0, \infty]$ para $A \in \mathscr{F}$. Isso segue por construção de $\nu$ e $f$. Além disso, $\nu(\varnothing)=\int_{\varnothing} f d \mu=\int_{\Omega}\left(f \mathbb{1}_{[\varnothing]}\right)(\omega) \mu(d \omega)=0$, pois $f \mathbb{1}_{[\varnothing]}$ é nula q.s. Finalmente, sejam $\left\{A_{k}\right\}$ uma sequência disjunta de conjuntos em $\mathscr{F}$ tal que $\bigcup_{k=1}^{\infty} A_{k} \in \mathscr{F}, g_{n} \doteq f \mathbb{1}_{\left[\bigcup_{k=1}^{n} A_{k}\right]}=\sum_{k=1}^{n} f \mathbb{1}_{\left[A_{k}\right]}$ e $g \doteq f \mathbb{1}_{\left[\bigcup_{k=1}^{\infty} A_{k}\right]}$. Temos que $0 \leq g_{n} \uparrow g$ e portanto, pelo teorema da convergência monótona, $\lim \int_{\Omega} g_{n} d \mu=\int_{\Omega} g d \mu$, ou seja, $\lim \int_{\Omega} f \mathbb{1}_{\left[\bigcup_{k=1}^{n} A_{k}\right]} d \mu=\lim \int_{\Omega} \sum_{k=1}^{n} f \mathbb{1}_{\left[A_{k}\right]}=\sum_{k=1}^{\infty} \int_{\Omega} f \mathbb{1}_{\left[A_{k}\right]} d \mu=$ $\sum_{k=1}^{\infty} \nu\left(A_{k}\right)=\int_{\Omega} \lim f \mathbb{1}_{\left[\bigcup_{k=1}^{n} A_{k}\right]} d \mu=\int_{\Omega} f \mathbb{1}_{\left[\bigcup_{k=1}^{\infty} A_{k}\right]} d \mu=\nu\left(\bigcup_{k=1}^{\infty} A_{k}\right)$.

Definição C.2. Dizemos que a medida $\nu: \mathscr{F} \rightarrow \mathbb{R}^{+}$definida por

$$
\nu(A)=\int_{A} f d \mu, \quad A \in \mathscr{F}
$$

tem densidade $f$ com respeito à medida $\mu$. 
Definição C.3. Sejam $\mu$ e $\nu$ medidas em $(\Omega, \mathscr{F})$. Dizemos que $\nu$ é absolutamente contínua com respeito a $\mu$ quando para cada $A \in \mathscr{F}, \mu(A)=0$ implicar que $\nu(A)=0$.

Observe que se $\nu$ tem densidade $f$ com respeito a $\mu$ então $\nu$ é absolutamente contínua com respeito a $\mu$. De fato, $\nu(A)=\int_{A} f d \mu=\int_{\Omega} f \mathbb{1}_{[A]} d \mu=0$ se $\mu(A)=0$, pois $f \mathbb{1}_{[A]} \equiv 0$ q.s.

O teorema de Radon-Nikodym é quase uma recíproca para essa observação. Antes,

Definição C.4. Seja $\mu$ uma medida em $(\Omega, \mathscr{F})$. Dizemos que $\mu$ é finita quando $\mu(\Omega)<\infty$, e que $\mu$ é $\sigma$-finita quando existir uma sequência $\left\{A_{k}\right\}, A_{k} \in \mathscr{F}$ finita ou enumerável tal que $\Omega=\bigcup A_{k} e$ $\mu\left(A_{k}\right)<\infty$.

Teorema C.5 (Radon-Nikodym). Se $\mu$ e $\nu$ são medidas $\sigma$-finitas e $\nu$ é absolutamente contínua com respeito a $\mu$ então existe $f$ não negativa, $\mathscr{F}$-mensurável tal que $\nu(A)=\int_{A} f d \mu$ para qualquer $A \in \mathscr{F}$. Isto é, $\nu$ tem densidade $f$ com respeito a $\mu$.

Para a demonstração, veja [1].

Definição C.6. Seja $X$ uma variável aleatória definida no espaço de probabilidade $(\Omega, \mathscr{F}, \mathrm{P}), \mathrm{P}$ integrável e $\mathscr{G}$ é uma sub- $\sigma$-álgebra em $\mathscr{F}$. Dizemos que a variável aleatória $\mathbf{E}(X \| \mathscr{G})$ é uma esperança condicional de $X$ dado $\mathscr{G}$, quando satisfazer as seguintes propriedades:

i) $\mathbf{E}(X \| \mathscr{G})$ é $\mathscr{G}$-mensurável e integrável.

ii) $\mathbf{E}(X \| \mathscr{G})$ satisfaz a seguinte equação funcional:

$$
\int_{G} \mathbf{E}(X \| \mathscr{G}) d \mathrm{P}=\int_{G} X d \mathrm{P}, \quad G \in \mathscr{G} .
$$

Proposição C.7. Existe uma variável aleatória $\mathbf{E}(X \| \mathscr{G})$ com as propriedades da definição anterior.

Demonstração. Inicialmente, considere $X$ não negativa. Defina uma medida $\nu$ em $(\Omega, \mathscr{G})$ por $\nu(G)=$ $\int_{G} X d \mathrm{P}$. Como $X$ é integrável, $\nu$ é finita e é absolutamente contínua com respeito a P. Pelo teorema de Radon-Nikodym, existe uma função $f, \mathscr{G}$-mensurável tal que $\nu(G)=\int_{G} f d \mathrm{P}$. Assim, da integrabilidade de $X$ segue a de $f$ e portanto essa $f$ satisfaz as propriedades (i) e (ii). Se $X$ não for necessáriamente não negativa, considere a decomposição $X=X^{+}-X^{-}$e obtenha $f^{+} \mathrm{e}$ $f^{-}$. A proposicão está provada pois $f^{+}-f^{-}$satisfazem as condições da definição anterior. Perceba 
que aqui nada se afirmou sobre a unicidade da esperança condicional. De fato, existem várias esperanças condicionais, basta alterar a função em conjuntos de medida P-nula. As propriedades continuam sendo satisfeitas e chamamos cada uma das esperanças condicionais de versão da esperança condicional.

Observe que se $\mathscr{G}=\{\emptyset, \Omega\}$ então a esperança condicional, por ser $\mathscr{G}$-mensurável, só pode ser constante. Disso, vem que $\int_{\Omega} \mathbf{E}(X \| \mathscr{G}) d \mathrm{P}=\mathbf{E}(X \| \mathscr{G}) \mathrm{P}(\Omega)=\mathbf{E}(X \| \mathscr{G})=\int_{\Omega} X d \mathrm{P}$ ou seja, $\mathbf{E}(X \| \mathscr{G})=\mathbf{E}(X)$, para quase todo $\omega \in \Omega$. Observe também que para $\mathscr{G}=\mathscr{F}, X$ satisfaz todas a propriedades donde $\mathbf{E}(X \| \mathscr{F})=X$ para quase todo $\omega \in \Omega$.

Teorema C.8. Sejam $\mathscr{G}$ uma sub- $\sigma$-àlgebra de $\mathscr{F}, \mathcal{I}$ um $\pi$-sistema* tal que $\sigma(\mathcal{I})=\mathscr{G}$ e $\Omega \in \mathcal{I}, X$ e $Y$ variavéis aleatórias em $\mathrm{L}^{1}$ com $Y$ sendo $\mathscr{G}$-mensurável. Então $Y=\mathbf{E}(X \| \mathscr{G})$ se e somente se $\mathbf{E}\left(Y \mathbb{1}_{A}\right)=\mathbf{E}\left(X \mathbb{1}_{A}\right)$ para qualquer $A \in \mathcal{I}$.

Demonstração. Se $Y=\mathbf{E}(X \| \mathscr{G})$, então pela definição de esperança condicional, vale que $\mathbf{E}\left(Y \mathbb{1}_{A}\right)=$ $\mathbf{E}\left(X \mathbb{1}_{A}\right)$ para qualquer $A \in \mathcal{I}$. Reciprocamente, suponha que $\mathbf{E}\left(Y \mathbb{1}_{A}\right)=\mathbf{E}\left(X \mathbb{1}_{A}\right)$ para qualquer $A \in \mathcal{I}$, devemos mostrar que $\mathbf{E}\left(Y \mathbb{1}_{A}\right)=\mathbf{E}\left(X \mathbb{1}_{A}\right)$ para qualquer $A \in \mathscr{G}$. Defina

$$
\mathcal{L} \doteq\left\{A \in \mathscr{F}: \mathbf{E}\left(Y \mathbb{1}_{A}\right)=\mathbf{E}\left(X \mathbb{1}_{A}\right)\right\}
$$

Vamos mostrar que $\mathscr{G} \subseteq \mathcal{L}$. De fato, da integrabilidade de $X$ e $Y$ e da linearidade da integral de Lebesgue, temos que $\mathcal{L}$ é um $\lambda$-sistema. Como por hipotése, $\mathcal{I} \subseteq \mathcal{L}$, pelo teorema da classe monótona ${ }^{\dagger}$ resulta que $\mathscr{G}=\sigma(\mathcal{I}) \subseteq \mathcal{L}$. Como queríamos.

O próximo teorema lista algumas propriedades da esperança condicional. Todas as igualdades e desigualdades valem para quase todo $\omega \in \Omega$. A prova destes resultados pode ser encontrada em [1] página 447. Daqui em diante, assume-se tacitamente que $X$ é uma variável aleatória definida no espaço $(\Omega, \mathscr{F}, \mathrm{P})$ e $\mathscr{G}$ é uma sub- $\sigma$-álgebra de $\mathscr{F}$.

Teorema C.9. Sejam $X, Y$ e $X_{n}$ variáveis aleatórias integráveis

i) Se $X=$ a para quase todo $\omega \in \Omega$, então $\mathbf{E}(X \| \mathscr{G})=a$.

ii) Se a e b são constantes, vale que $\mathbf{E}(a X+b Y \| \mathscr{G})=a \mathbf{E}(X \| \mathscr{G})+b \mathbf{E}(Y \| \mathscr{G})$.

\footnotetext{
* Veja a definição A.1 na página 151. † Veja o teorema A.2 na página 151.
} 
iii) Se $X \leq Y$ para quase todo $\omega \in \Omega$, então $\mathbf{E}(X \| \mathscr{G}) \leq \mathbf{E}(Y \| \mathscr{G})$.

iv) $|\mathbf{E}(X|| \mathscr{G})| \leq \mathbf{E}(|X||| \mathscr{G})$.

v) Se $\lim _{n} X_{n}=X$ para quase todo $\omega \in \Omega,\left|X_{n}\right| \leq Y$ e se $Y$ for integrável, então $\lim _{n} \mathbf{E}\left(X_{n} \| \mathscr{G}\right)=$ $\mathbf{E}(X \| \mathscr{G})$ para quase todo $\omega \in \Omega$.

Teorema C.10. Suponha que $X$ éĞGensurável e que $Y$ e $X Y$ sejam integráveis então, para quase todo $\omega \in \Omega$ vale que

$$
\mathbf{E}(X Y \| \mathscr{G})=X \mathbf{E}(Y \| \mathscr{G})
$$

Demonstração. Inicialmente, sejam $G_{0} \in \mathscr{G}$ e $X=\mathbb{1}_{\left[G_{0}\right]}$. Temos que $\mathbb{1}_{\left[G_{0}\right]} \mathbf{E}(Y \| \mathscr{G})$ é $\mathscr{G}$-mensurável e integrável (pois $\mathbb{1}_{\left[G_{0}\right]} \mathbf{E}(Y \| \mathscr{G}) \leq \mathbf{E}(Y \| \mathscr{G})$ ). Além disso, como vale que $\int_{G \cap G_{0}} \mathbf{E}(Y \| \mathscr{G}) d \mathrm{P}=$ $\int_{G \cap G_{0}} Y d \mathrm{P}$, vem que $\int_{G} \mathbb{1}_{\left[G_{0}\right]} \mathbf{E}(Y \| \mathscr{G}) d \mathrm{P}=\int_{G} \mathbb{1}_{\left[G_{0}\right]} Y d \mathrm{P}=\int_{G} \mathbf{E}\left(\mathbb{1}_{\left[G_{0}\right]} Y \| \mathscr{G}\right) d \mathrm{P}$. Resulta portanto que $X \mathbf{E}(Y \| \mathscr{G})$ é uma versão de $\mathbf{E}(X Y \| \mathscr{G})$ quando $X=\mathbb{1}_{\left[G_{0}\right]}$.

Agora para $X=\sum_{k=1}^{n} x_{k} \mathbb{1}_{\left[G_{k}\right]}, \operatorname{com} G_{1}, \ldots, G_{n} \in \mathscr{G}$ e $x_{1}, \ldots, x_{n}$ constantes reais, temos pelo o que acabamos de mostrar e pelo item (ii) do Teorema C.9 que:

$$
\begin{aligned}
\int_{G} \mathbf{E}(X Y \| \mathscr{G}) d \mathrm{P} & =\int_{G} \mathbf{E}\left(x_{1} \mathbb{1}_{\left[A_{1}\right]} Y+\cdots+x_{n} \mathbb{1}_{\left[A_{n}\right]} Y \| \mathscr{G}\right) d \mathrm{P} \\
& =\int_{G} x_{1} \mathbf{E}\left(\mathbb{1}_{\left[A_{1}\right]} Y \| \mathscr{G}\right) d \mathrm{P}+\cdots+x_{n} \int_{G} \mathbf{E}\left(\mathbb{1}_{\left[A_{n}\right]} Y \| \mathscr{G}\right) d \mathrm{P} \\
& =\int_{G} x_{1} \mathbb{1}_{\left[A_{1}\right]} \mathbf{E}(Y \| \mathscr{G}) d \mathrm{P}+\cdots+\int_{G} x_{n} \mathbb{1}_{\left[A_{n}\right]} \mathbf{E}(Y \| \mathscr{G}) d \mathrm{P} \\
& =\int_{G} X \mathbf{E}(Y \| \mathscr{G}) d \mathrm{P} \\
& =\int_{G} X Y d \mathrm{P} .
\end{aligned}
$$

Como $X \mathbf{E}(Y \| \mathscr{G})$ é $\mathscr{G}$-mensurável e integrável, segue que $X \mathbf{E}(Y \| \mathscr{G})$ é uma versão de $\mathbf{E}(X Y \| \mathscr{G})$ para $X$ simples.

Finalmente, seja $X$ arbitrária e $\mathscr{G}$-mensurável. Sabemos que existe uma sequência $\left\{X_{n}\right\}$ de funções simples, $\mathscr{G}$-mensuráveis tais que $\left|X_{n}\right|<|X|$ e $\lim _{n} X_{n}=X$. Como $\left|X_{n} Y\right| \leq|X Y|$ e $|X Y|$ 
é integrável por hipótese, pelo item (v) do Teorema C.9, temos que $\lim _{n} \mathbf{E}\left(X_{n} Y \| \mathscr{G}\right)=\mathbf{E}(X Y \| \mathscr{G})$ para quase todo $\omega \in \Omega$. Resulta portanto que $X \mathbf{E}(Y \| \mathscr{G})=\lim _{n} X_{n} \mathbf{E}(Y \| \mathscr{G})=\lim _{n} \mathbf{E}\left(X_{n} Y \| \mathscr{G}\right)=$ $\mathbf{E}(X Y \| \mathscr{G})$. Como $X \mathbf{E}(Y \| \mathscr{G})$ é $\mathscr{G}$-mensurável (pois é limite de funções $\mathscr{G}$-mensuráveis) e é integrável (pois, pelos itens (iii) e (iv) do teorema C.9, $\left|X_{n} \mathbf{E}(Y|| \mathscr{G})\right|=\mid \mathbf{E}\left(X_{n} Y|| \mathscr{G}\right) \leq \mathbf{E}\left(\left|X_{n} Y\right| \| \mathscr{G}\right) \leq$ $\mathbf{E}(|X Y| \| \mathscr{G})$, que é integrável), resulta que $X \mathbf{E}(Y|| \mathscr{G})$ é uma versão de $\mathbf{E}(X Y \| \mathscr{G})$, como queríamos.

A próxima propriedade da esperança condicional é conhecida como propriedade da torre.

Teorema C.11. Sejam $X$ uma variável aleatória integrável e $\mathscr{G}_{1} \subset \mathscr{G}_{2}$, sub- $\sigma$-álgebras de $\mathscr{F}$. Então para quase todo $\omega \in \Omega$ vale que

$$
\mathbf{E}\left(\mathbf{E}\left(X \| \mathscr{G}_{2}\right) \| \mathscr{G}_{1}\right)=\mathbf{E}\left(X \| \mathscr{G}_{1}\right)=\mathbf{E}\left(\mathbf{E}\left(X \| \mathscr{G}_{1}\right) \| \mathscr{G}_{2}\right)
$$

Demonstração. Para a primeira igualdade, temos que $\mathbf{E}\left(X \| \mathscr{G}_{2}\right)$ é integrável portanto, pela definição de esperança condicional, $\mathbf{E}\left(\mathbf{E}\left(X \| \mathscr{G}_{2}\right) \| \mathscr{G}_{1}\right)$ é $\mathscr{G}_{1}$-mensurável e integrável, assim falta verificar o item (ii) da definição C.6 isto é, $\int_{G} \mathbf{E}\left(\mathbf{E}\left(X \| \mathscr{G}_{2}\right) \| \mathscr{G}_{1}\right) d \mathrm{P}=\int_{G} X d \mathrm{P}$ para $G \in \mathscr{G}_{1}$. De fato, se $G \in \mathscr{G}_{1}$ então $G \in \mathscr{G}_{2}$, donde $\int_{G} \mathbf{E}\left(\mathbf{E}\left(X \| \mathscr{G}_{2}\right) \| \mathscr{G}_{1}\right) d \mathrm{P}=\int_{G} \mathbf{E}\left(X \| \mathscr{G}_{2}\right) d \mathrm{P}=\int_{G} X d \mathrm{P}$. Para a segunda, observe que $\mathbf{E}\left(X|| \mathscr{G}_{1}\right)$ por ser $\mathscr{G}_{1}$-mensurável é também $\mathscr{G}_{2}$-mensurável, assim pela observação após a prova da proposição C.7 segue o que queríamos.

Teorema C.12. Seja $X$ uma variável aleatória integrável e $\mathscr{G}$ uma sub- $\sigma$-álgebra de $\mathscr{F}$. Se $X$ for independente de $\mathscr{G}$ então, com probabilidade 1 , vale que

$$
\mathbf{E}(X \| \mathscr{G})=\mathbf{E}(X) .
$$

Demonstração. Lembramos o leitor que dizer que $X$ é independente de $\mathscr{G}$ é dizer que a $\sigma$-álgebra gerada por $X$ é independente de $\mathscr{G}$. Note que como $\mathbf{E}(X)$ é constante, segue que $\mathbf{E}(X) \in \mathscr{G}$. Agora 
seja $\Lambda \in \mathscr{G}$ arbitrário. Temos que

$$
\begin{aligned}
\int_{\Lambda} \mathbf{E}(X) d \mathrm{P} & =\mathbf{E}(X) \mathrm{P}(\Lambda) \\
& =\mathbf{E}(X) \mathbf{E}\left(\mathbb{1}_{[\Lambda]}\right) \\
& =\mathbf{E}\left(X \cdot \mathbb{1}_{[\Lambda]}\right) \\
& =\int_{\Omega} X \cdot \mathbb{1}_{[\Lambda]} d \mathrm{P} \\
& =\int_{\Lambda} X d \mathrm{P} .
\end{aligned}
$$

e assim, provamos o que queríamos.

O próximo resultado é a chamada Desigualdade de Jensen Condicional. A demonstração pode ser encontrada em [1].

Teorema C.13. Seja $X$ uma variável aleatória e $\varphi$ uma função convexa definida em $\mathbb{R}$ tal que a variável aleatória $\varphi(X)$ seja integrável. Então, para $\mathscr{G}$ sub- $\sigma$-àlgebra arbitrária de $\mathscr{F}$, vale que

$$
\varphi(\mathbf{E}(X \| \mathscr{G})) \leq \mathbf{E}(\varphi(X) \| \mathscr{G})
$$

para quase todo $\omega \in \Omega$.

Corolário C.14. Seja $X$ uma variável aleatória e $\mathcal{G}$ uma sub- $\sigma$-àlgebra de $\mathscr{F}$. Então

$$
|\mathbf{E}(X|| \mathscr{G})| \leq \mathbf{E}(|X| \| \mathscr{G}),
$$

para quase todo $\omega \in \Omega$. Se $X \in \mathrm{L}_{p}(\Omega, \mathscr{F}, \mathrm{P})$ para algum $p \in[1, \infty)$, então

$$
\mathbf{E}(|X| \| \mathscr{G}) \leq \mathbf{E}\left(|X|^{p}|| \mathscr{G}\right)^{\frac{1}{p}}
$$

para quase todo $\omega \in \Omega$ e também

$$
\|\mathbf{E}(X \| \mathscr{G})\|_{p} \leq\|\mathbf{E}(|X| \| \mathscr{G})\|_{p} \leq\|X\|_{p}
$$


Demonstração. Como $x \mapsto|x|, x \in \mathbb{R}$ é uma função convexa em $\mathbb{R},($ C.1) segue imeditamente do teorema anterior. Agora, suponha que $X \in \mathrm{L}_{p}(\Omega, \mathscr{F}, \mathrm{P})$ para algum $p \in[1, \infty)$. Como omo $x \mapsto|x|$, $x \in \mathbb{R}$ é uma função convexa em $\mathbb{R}$. Segue pelo teorema anterior que

$$
|\mathbf{E}(X|| \mathscr{G})|^{p} \leq \mathbf{E}\left(|X|^{p}|| \mathscr{G}\right)
$$

e portanto

$$
|\mathbf{E}(X|| \mathscr{G})| \leq \mathbf{E}\left(|X|^{p}|| \mathscr{G}\right)^{\frac{1}{p}}
$$

para quase todo $\omega \in \Omega$. Logo

$$
|\mathbf{E}(|X||| \mathscr{G})|=\mathbf{E}(|X||| \mathscr{G}) \leq \mathbf{E}\left(|X|^{p}|| \mathscr{G}\right)^{\frac{1}{p}}
$$

para quase todo $\omega \in \Omega$ e portanto (C.2) é válido. Para a última desigualdade, usando as duas primeiras e a monotonicidade da integral de Lebesgue, temos que

$$
\int_{\Omega}|\mathbf{E}(X|| \mathscr{G})|^{p} d \mathrm{P} \leq \int_{\Omega} \mathbf{E}(|X| \| \mathscr{G})^{p} d \mathrm{P} \leq \int_{\Omega} \mathbf{E}\left(|X|^{p} \| \mathscr{G}\right) d \mathrm{P} .
$$

Mas, pela definição de esperança condicional, $\int_{\Omega} \mathbf{E}\left(|X|^{p}|| \mathscr{G}\right) d \mathrm{P}=\int_{\Omega}|X|^{p} d \mathrm{P}$ e portanto

$$
\int_{\Omega}|\mathbf{E}(X|| \mathscr{G})|^{p} d \mathrm{P} \leq \int_{\Omega} \mathbf{E}(|X||| \mathscr{G})^{p} d \mathrm{P} \leq \int_{\Omega}|X|^{p} d \mathrm{P} .
$$

Elevando todos os membros a $1 / p$, resulta (C.3).

O próximo teorema indica que a experança condicional é contínua sob a norma dos espaços $\mathrm{L}^{p}$, $p \in[1, \infty)$.

Teorema C.15. Seja $\left\{X_{n}\right\}$ uma sequência de variáveis aleatórias em $\mathrm{L}^{p}(\Omega, \mathscr{F}, \mathrm{P})$ para algum $p \in$ $[1, \infty)$. Se $\lim _{n \rightarrow \infty} X_{n}=X$ em $\mathrm{L}^{p}$, então

$$
\lim _{n \rightarrow \infty} \mathbf{E}\left(X_{n} \| \mathscr{G}\right)=\mathbf{E}(X \| \mathscr{G})
$$

em $\mathrm{L}^{p}$, para qualquer sub- $\sigma$-àlgebra $\mathscr{G}$ de $\mathscr{F}$. 
Demonstração. Por (C.3) do corolário anterior, temos que

$$
\left\|\mathbf{E}\left(X_{n} \| \mathscr{G}\right)\right\|_{p} \leq\left\|X_{n}\right\|_{p}<\infty
$$

e portanto $\mathbf{E}\left(X_{n} \| \mathscr{G}\right) \in \mathrm{L}^{p}$ para todo $n \in \mathbb{N}$. Pelo mesmo motivo, segue que $\mathbf{E}(X \| \mathscr{G}) \in \mathrm{L}^{p}$. Pela propriedade iii do Teorema C.9 e por (C.3) do corolário anterior temos

$$
\left\|\mathbf{E}\left(X_{n} \| \mathscr{G}\right)-\mathbf{E}(X \| \mathscr{G})\right\|_{p}=\left\|\mathbf{E}\left(X_{n}-X \| \mathscr{G}\right)\right\|_{p} \leq\left\|X_{n}-X\right\|_{p}
$$

e portanto, como $\lim _{n \rightarrow \infty}\left\|X_{n}-X\right\|_{p}=0$ resulta que

$$
\lim _{n \rightarrow \infty}\left\|\mathbf{E}\left(X_{n} \| \mathscr{G}\right)-\mathbf{E}(X \| \mathscr{G})\right\|_{p}=0
$$

Como queríamos.

O próximo teorema é o lema de Fatou para esperanças condicionais. Uma demonstração pode ser encontrada em [28].

Teorema C.16. Seja $\left\{X_{n}\right\}_{n \geq 1}$ uma sequência de variáveis aleatórias integráveis, reais estendidas, definidas no espaço de probabilidade $(\Omega, \mathscr{F}, \mathrm{P})$ e $\mathscr{G}$ uma sub- $\sigma$-àlgebra de $\mathscr{F}$. Se $\liminf _{n \rightarrow \infty} X_{n}$ for integrável e se existir uma variável aleatória integrável e real estendida $X$ tal que para todo $n \geq 1$ valer que $X_{n} \geq X$, P-q.s., então

$$
\mathbf{E}\left(\liminf _{n \rightarrow \infty} X_{n} \| \mathscr{G}\right) \leq \liminf _{n \rightarrow \infty} \mathbf{E}\left(X_{n} \| \mathscr{G}\right)
$$

P-q. s. 


\title{
Apêndice D
}

\section{Uma construção do movimento Browniano}

\author{
Reason's last step is the recognition that there are \\ an infinite number of things which are beyond it. \\ Blaise Pascal
}

\section{D.1 Introdução}

Segundo Tyge Nielsen [19], o componente básico da construção de $98 \%$ de toda a teoria de finanças em tempo contínuo é um movimento Browniano. No que se segue, fixamos um espaço de probabilidade filtrado $\left(\Omega, \mathscr{F},\left\{\mathscr{F}_{t}\right\}, \mathrm{P}\right)$ e seja $T \in(0,+\infty]$. A definição deste processo é a seguinte

Definição D.1. Dizemos que um processo estocástico $\left\{B_{t}: t \in(0, T]\right\}$ é um movimento Browniano padrão em $(0, T]$, quando estão satisfeitas as seguintes condições:

(B1) $B_{0}=0$.

(B2) Para qualquer sequência finita $0 \leq t_{1}<\ldots<t_{n}<T$, as variáveis aleatórias

$$
B_{t_{2}}-B_{t_{1}}, B_{t_{3}}-B_{t_{2}}, \ldots, B_{t_{n}}-B_{t_{n-1}}
$$

são independentes. Ou seja, dizemos que o movimento Browniano possui incrementos independentes.

(B3) Para quaisquer $0 \leq s \leq t<T, B_{t}-B_{s} \sim N(0, t-s)$. 
(B4) $B_{t}(\omega)$ é uma função contínua, $\mathrm{P}-\mathrm{q}$.s. Dizemos também que $B_{t}$ tem trajetórias contínuas $\mathrm{P}-\mathrm{q} . \mathrm{s}$.

Nosso objetivo será provar que existe um processo $\left\{B_{t}: t \in(0,+\infty]\right\}$, satisfazendo (B1) - (B4).

Existe mais de uma maneira de provar isso. Apresentaremos aqui a construção de Lévy-Ciesielski. O plano será provar primeiro a existência de um processo satisfazendo (B1) - (B4) em $(0,1]$ e depois extende-lo para $(0,+\infty]$ de tal forma que as propriedades $(\mathrm{B} 1)-(\mathrm{B} 4)$ continuem valendo. Antes, faremos uma recordação de alguns fatos sobre a distribuição Normal e análise funcional que serão usados nesta construção.

\section{D.2 Fatos sobre a distribuição Normal e processos Gaussianos}

Seja $V=\left(V_{1}, V_{2}, \ldots, V_{d}\right)^{T}$ um vetor aleatório, lembramos que a média de $V$ é o vetor $\mu$ dado por

$$
\mu=\mathbb{E}[V]=\left(\begin{array}{c}
\mathbb{E}\left[V_{1}\right] \\
\mathbb{E}\left[V_{2}\right] \\
\vdots \\
\mathbb{E}\left[V_{d}\right]
\end{array}\right)
$$

e a matriz de covariância $\Sigma$ de $V$ é dada por

$$
\Sigma=\left(\begin{array}{ccc}
\sigma_{11} & \cdots & \sigma_{1 d} \\
\vdots & \ddots & \vdots \\
\sigma_{d 1} & \cdots & \sigma_{d d}
\end{array}\right)
$$

aqui $\sigma_{i j}=\operatorname{Cov}\left(V_{i}, V_{j}\right) \doteq \mathbb{E}\left[\left(V_{i}-\mathbb{E}\left[V_{i}\right]\right)\left(V_{j}-\mathbb{E}\left[V_{j}\right]\right)\right]$.

Uma maneira de caracterizar a distribuição de um vetor aleatório, é através do conceito de função característica.

Definição D.2. Sejam $V=\left(V_{1}, V_{2}, \ldots, V_{d}\right)$ um vetor aleatório e $\theta=\left(\theta_{1}, \theta_{2} \ldots, \theta_{d}\right)$ um vetor de números reais. Definimos a função característica de $V$ como a função $\phi: \mathbb{R}^{d} \rightarrow \mathbb{C}$, dada por $\phi(\theta)=$ $\mathbb{E}\left[\exp \left(i \theta^{T} V\right)\right]$.

Prova-se (veja [4], por exemplo) que conhecendo a função característica de um vetor aleatório, determina-se a sua distribuição. Em particular, temos o seguinte resultado 
Proposição D.3. Um vetor aleatório $V$ tem distribuição Normal multivariada, com vetor de médias $\mu$ e matriz de covariância $\Sigma$, se e somente se, para qualquer $\theta \in \mathbb{R}^{d}$

$$
\phi(\theta)=\exp \left(\imath \theta^{T} \mu-\frac{1}{2} \theta^{T} \Sigma \theta\right) .
$$

Em particular, uma variável aleatória é Normal com média $\mu$ e variância $\sigma^{2}$, se e somente se, para qualquer $t \in \mathbb{R}$

$$
\phi(t)=\exp (\imath t \mu) \exp \left(-t^{2} \sigma^{2} / 2\right)
$$

Definimos também a função geradora de momentos de uma váriavel aleatória $X$ por

$$
m(t) \doteq \mathbf{E}(\exp (t X))
$$

na condição que exp $(t X)$ seja integrável para $t$ em uma vizinhança de 0. Pode-se mostrar que, assim como a função característica, a função geradora de momentos determina univocamente a distribuição de uma variável aleatória. Mostra-se também que a função geradora de momentos de uma variável aleatória $X$ Normal com média $\mu$ e variância $\sigma^{2}$ é $\exp \left(\mu t+(\sigma t)^{2} / 2\right)$. Em outras palavras,

$$
X \sim N\left(\mu, \sigma^{2}\right) \Leftrightarrow m(t)=\exp \left(\mu t+(\sigma t)^{2} / 2\right)
$$

Para o caso multidimensional, definimos a função geradora de momentos de vetor aleatório $V$ como

$$
M(t) \doteq \mathbf{E}\left(\exp \left(\sum_{i=1}^{d} t_{i} X_{i}\right)\right)
$$

onde $t \in \mathbb{R}^{d}$. Além disso, temos a seguinte caracterização:

$$
V \sim N(\mu, \Sigma) \Leftrightarrow M(t)=\exp \left(\mu t^{T}-\frac{1}{2} t \Sigma t^{T}\right)
$$

Observamos que uma vantagem de se usar a função característica ao invés da geradora de momentos é que ela está definida para qualquer variavél aleatória $X$ pois exp (itx) é limitada para todo $t \in \mathbb{R}$.

Uma outra caracterização de um vetor aleatório com distribuição Normal multivariada é dada por uma relação com as coordenadas que o compõem. 
Proposição D.4. Um vetor aleatório d-dimensional $\left(V_{1}, V_{2}, \ldots, V_{d}\right)$ tem distribuição Normal multivariada, se e somente se, qualquer combinação linear $\theta_{1} V_{1}+\theta_{2} V_{2}+\cdots+\theta_{d} V_{d}$ for uma variável aleatória de distribuição Normal.

Verificar se variavéis aleatórias são independentes pode ser algo complicado em geral. Mas, se estas tiverem distribuição Normal o trabalho é simplificado por causa do próximo resultado

Proposição D.5. Seja $V=\left\{V_{1}, V_{2}, \ldots, V_{d}\right\}$ um vetor aleatório. Então, as coordenadas desse vetor são independentes se e somente se, a matriz de covariância for diagonal.

A próxima desigualdade será importante na finalização da construção do movimento Browniano.

Lema D.6. Seja $X \sim N(0,1)$ e c uma constante positiva. Então vale que

$$
\mathrm{P}[|X|>c]<\frac{2}{c \sqrt{2 \pi}} \exp \left(-\frac{1}{2} c^{2}\right)
$$

Demonstração. Como $x / c>1$, temos, para $x>c$

$$
\begin{aligned}
\mathrm{P}[|X|>c] & =\frac{1}{\sqrt{2 \pi}} \int_{c}^{\infty} \exp \left(-\frac{x^{2}}{2}\right) d x \\
& <\frac{1}{\sqrt{2 \pi}} \int_{c}^{\infty} \frac{x}{c} \exp \left(-\frac{x^{2}}{2}\right) d x \\
& =\frac{1}{c \sqrt{2 \pi}} \exp \left(-\frac{c^{2}}{2}\right) .
\end{aligned}
$$

Por simetria, $\mathrm{P}[|X|>c]=2 \mathrm{P}[X>c]$, e assim, provamos o que queríamos.

Um conceito importante, que estará permeado neste texto é o tema da próxima

Definição D.7. Dizemos que um processo estocástico $\left\{X_{t}: t \in[0, T)\right\}$ é um processo Gaussiano, quando para qualquer sequência finita $0 \leq t_{1}<\ldots<t_{n}<T$, o vetor $\left(X_{t_{1}}, X_{t_{2}}, \ldots, X_{t_{n}}\right)$ tem distribuição Normal multivariada.

O próximo resultado, liga os conceitos acima e mostra como identificar processos Gaussianos com incrementos independentes. 
Teorema D.8. Se um processo gaussiano $\left\{X_{t}: t \in[0, T)\right\}$ é tal que $\mathbb{E}\left[X_{t}\right]=0$ para qualquer $t \in$ $[0, T)$, e $\operatorname{Cov}\left(X_{s}, X_{t}\right)=s \wedge t$, para quaisquer $0 \leq s, t<T$, então o processo $\left\{X_{t}\right\}$ tem incrementos independentes.

Demonstração. De acordo com a Proposição D.5, devemos provar que dada uma seqûencia arbitrária $0 \leq t_{1}<t_{2}<\ldots<t_{n}<T$, a matriz de covariância do vetor $\left(X_{t_{2}}-X_{t_{1}}, X_{t_{3}}-X_{t_{2}}, \ldots, X_{t_{n}}-X_{t_{n-1}}\right)$ é diagonal. De fato, sejam $i<j$. Temos que

$$
\begin{aligned}
\sigma_{i j} & =\mathbb{E}\left[\left(X_{t_{i}}-X_{t_{i-1}}\right)\left(X_{t_{i}}-X_{t_{i-1}}\right)\right] \\
& =\mathbb{E}\left[X_{t_{i}} X_{t_{j}}\right]-\mathbb{E}\left[X_{t_{i}} X_{t_{j-1}}\right]-\mathbb{E}\left[X_{t_{i-1}} X_{t_{j}}\right]+\mathbb{E}\left[X_{t_{i-1}} X_{t_{j-1}}\right] \\
& =\operatorname{Cov}\left(X_{t_{i}}, X_{t_{j}}\right)-\operatorname{Cov}\left(X_{t_{i}}, X_{t_{j-1}}\right)-\operatorname{Cov}\left(X_{t_{i-1}}, X_{t_{j}}\right)+\operatorname{Cov}\left(X_{t_{i-1}}, X_{t_{j-1}}\right) \\
& =t_{i}-t_{i}-t_{i-1}+t_{i-1} \\
& =0 .
\end{aligned}
$$

Como queríamos.

O próximo teorema, fornece uma caracterização dos processos que satisfazem as três primeiras propriedades que definem o movimento Browniano. É essa a caracterização que vamos usar adiante, para provar a existência do mesmo.

Teorema D.9. Seja $\left\{X_{t}: t \in[0, T)\right\}$ um processo tal que $X_{0}=0$ e para uma seqûencia arbitrária $0 \leq t_{1}<t_{2}<\ldots<t_{n}<T, V=\left(X_{t_{1}}, X_{t_{2}}, \ldots, X_{t_{n}}\right)$ é tal que para qualquer $\theta \in \mathbb{R}^{n}$

$$
\phi(\theta)=\exp \left(-\frac{1}{2} \sum_{j=1}^{n} \sum_{k=1}^{n} \theta_{j} \theta_{k}\left(t_{j} \wedge t_{k}\right)\right)
$$

então, $\left\{X_{t}\right\}$ satisfaz as propriedades (B1),(B2) e (B3) da Definição D.1.

Demonstração. Em primeiro lugar, (B1) está satisfeito, pois por hipótese, $X_{0}=0$. Agora, de acordo com a Proposição D.3, temos que $\left\{X_{t}\right\}$ é um processo Gaussiano (veja a Definição D.7) com vetor de média $\mu$ nulo e matriz de covariância tal que $\sigma_{i j}=t_{i} \wedge t_{j}$, para quaisquer $0 \leq t_{i}, t_{j}<T$. Assim, as hipotéses do Teorema D.8 estão satisfeitas e portanto $\left\{X_{t}\right\}$ tem incrementos independentes. Provamos portanto que (B2) também está satisfeita. Finalmente, como $\left\{X_{t}\right\}$ é um processo Gaussiano, temos 
pela Proposição D.4 que tomando $s$ e $t$, tais que $0 \leq s<t<T$, a combinação linear $X_{t}-X_{s}$ é uma variável aleatória Normal. Agora, $\mathbb{E}\left[X_{t}-X_{s}\right]=\mathbb{E}\left[X_{t}\right]-\mathbb{E}\left[X_{s}\right]=0-0=0$ e $\operatorname{Var}\left(X_{t}-X_{s}\right) \doteq$ $\mathbb{E}\left[\left(X_{t}-X_{s}\right)^{2}\right]-\left(\mathbb{E}\left[\left(X_{t}-X_{s}\right)\right]\right)^{2}=\mathbb{E}\left[\left(X_{t}-X_{s}\right)^{2}\right]$. Mas

$$
\begin{aligned}
\mathbb{E}\left[\left(X_{t}-X_{s}\right)^{2}\right] & =\mathbb{E}\left[X_{t} X_{t}\right]-2 \mathbb{E}\left[X_{t} X_{s}\right]+\mathbb{E}\left[X_{s} X_{s}\right] \\
& =\operatorname{Cov}\left(X_{t}, X_{t}\right)-2 \operatorname{Cov}\left(X_{t}, X_{s}\right)+\operatorname{Cov}\left(X_{s}, X_{s}\right) \\
& =t-2 s+s \\
& =t-s .
\end{aligned}
$$

Provamos que $X_{t}-X_{s} \sim N(0, t-s)$, ou seja, (B3) está satisfeita. Como queríamos.

Corolário D.10. Se $\left\{X_{t}: t \in[0, T)\right\}$ é um processo Gaussiano, com $\mathbb{E}\left[X_{t}\right]=0$ para qualquer $t \in[0, T)$ e $\operatorname{Cov}\left(X_{t}, X_{s}\right)=t \wedge s$, para quaisquer $0 \leq t, s<T$ e tal que $X_{0}=0$, então, $\left\{X_{t}\right\}$ satisfaz as propriedades (B1), (B2) e (B3) da Definição D.1.

Demonstração. Imediata. Basta ver que pela Proposição D.3, o processo $\left\{X_{t}\right\}$ satisfaz as hipóteses do teorema anterior.

\section{D.3 Conceitos de Análise Funcional}

No que se segue, considere o espaço de Hilbert $\mathfrak{H} \doteq \mathrm{L}^{2}([0,1], \mathrm{B}([0,1]), \lambda)$, onde $\lambda$ é a medida de Lebesgue e $\mathrm{B}([0,1])$ são os borelianos do intervalo $[0,1]$. Para $f, g \in \mathfrak{H}$, definimos uma norma e um produto interno em $\mathfrak{H}$ respectivamente por

$$
\|f\| \doteq\left(\int_{0}^{1} f^{2}(t) \lambda(d t)\right)^{1 / 2} \mathrm{e}<f, g>\doteq \int_{0}^{1} f(t) g(t) \lambda(d t) .
$$

Definição D.11. Seja $\Psi=\left\{\psi_{1}, \psi_{2}, \ldots\right\}$ um conjunto enumerável de funções ortonormais em $\mathfrak{H}$, isto é, $\left\|\psi_{i}\right\|=1$, para qualquer $i$ e $<\psi_{i}, \psi_{j}>=0$, para quaisquer $i \neq j$. Dizemos que $\Psi$ é uma base ortonormal completa para $\mathfrak{H}$, quando para qualquer $f \in \mathfrak{H}$ vale que

$$
\lim _{n \rightarrow \infty} \sum_{i=1}^{n}<f, \psi_{i}>\psi_{i}=f, e m \mathrm{~L}^{2}
$$


ou seja,

$$
\lim _{n \rightarrow \infty}\left\|\sum_{i=1}^{n}<f, \psi_{i}>\psi_{i}-f\right\|=0 .
$$

Proposição D.12. Sejam $\Psi$ uma base ortonormal completa para $\mathfrak{H}$ e $f, g \in \mathfrak{H}$. Então valem

$$
\|f\|^{2}=\sum_{i=1}^{\infty}\left(<f, \psi_{i}>\right)^{2}
$$

$e$

$$
<f, g>=\sum_{i=1}^{\infty}<f, \psi_{i}><g, \psi_{i}>
$$

A relação (D.1) é conhecida como a identidade de Parseval.

\section{D.4 A construção do movimento Browniano em $[0,1]$}

\section{D.4.1 As três primeiras propriedades}

Em primeiro lugar, pode-se provar (veja [1]) que o espaço de probabilidade fixado $(\Omega, \mathscr{F}, \mathrm{P})$ pode ser tomado como um onde existe uma sequência de variaveis aleatórias independentes e identicamente distribuidas (i.i.d.), $X_{i} \sim N(0,1)$.

Teorema D.13. Seja $\left\{\psi_{i}\right\}$ uma base ortonormal completa de $\mathfrak{H}$ e $\left\{X_{i}\right\}$ uma sequência de variáveis aleatórias i.i.d, $X_{i} \sim N(0,1)$, para todo $i$. Defina

$$
W_{t}^{n}=\sum_{i=1}^{n} X_{i} \int_{0}^{t} \psi_{i}(\xi) \lambda(d \xi) .
$$

Então, para cada $t \in[0,1],\left\{W_{t}^{n}\right\}$ é uma sequência de Cauchy em $\mathrm{L}^{2}(\Omega, \mathscr{F}, \mathrm{P})$.

Demonstração. Defina

$$
I_{t}(\xi) \doteq\left\{\begin{array}{l}
1, \xi<t \\
0, \xi \geq t
\end{array}\right.
$$


Assim, podemos escrever

$$
\int_{0}^{t} \psi_{i}(\xi) \lambda(d \xi)=\int_{0}^{1} \psi_{i}(\xi) I_{t}(\xi) \lambda(d \xi)=<I_{t}, \psi_{i}>
$$

Como $\left\{\psi_{i}\right\}$ é completa, pelo o que vimos na seção anterior, são válidas as relações

$$
I_{t}=\sum_{i=1}^{\infty}<I_{t}, \psi_{i}>\psi_{i}
$$

e

$$
t=\left\|I_{t}\right\|^{2}=\sum_{i=1}^{\infty}\left(<I_{t}, \psi_{i}>\right)^{2}
$$

Agora, para $n>m$, temos

$$
\left\|W_{t}^{n}-W_{t}^{m}\right\|=\mathbb{E}\left[\left(W_{t}^{n}-W_{t}^{m}\right)^{2}\right]=\mathbb{E}\left[\left(\sum_{i=m+1}^{n} X_{i} \int_{0}^{t} \psi_{i}(\xi) \lambda(d \xi)\right)^{2}\right] .
$$

Mas, como a soma de variavéis aleatórias Normais independentes com média 0 e variância $\left(\int_{0}^{t} \psi_{i}(\xi) \lambda(d \xi)\right)^{2}$ é Normal com média 0 e variância dada pela soma das variâncias individuais, temos que

$$
\mathbb{E}\left[\left(\sum_{i=m+1}^{n} X_{i} \int_{0}^{t} \psi_{i}(\xi) \lambda(d \xi)\right)^{2}\right]=\sum_{i=m+1}^{n}\left(\int_{0}^{t} \psi_{i}(\xi) \lambda(d \xi)\right)^{2}=\sum_{i=m+1}^{n}\left(<I_{t}, \psi_{i}>\right)^{2}
$$

onde a última igualdade é válida pela relação (D.3). Agora, por (D.5), temos que $\sum_{i=1}^{\infty}\left(<I_{t}, \psi_{i}>\right)^{2}$ é convergente e portanto, para $n, m \rightarrow \infty$, resulta que

$$
\sum_{i=m+1}^{n}\left(<I_{t}, \psi_{i}>\right)^{2} \rightarrow 0 .
$$

Assim, de (D.6), (D.7) e (D.8), resulta que

$$
\left\|W_{t}^{n}-W_{t}^{m}\right\| \rightarrow 0
$$


quando $n, m \rightarrow \infty$, ou seja, a seqûencia é de Cauchy.

Teorema D.14. Nas condições do último teorema, o processo $\left\{W_{t}: t \in[0,1]\right\}$ é Gaussiano com vetor de média nulo e matriz de covariância tal que $\sigma_{i j}=t_{i} \wedge t_{j}$, para quaisquer $0 \leq t_{i}, t_{j}<1$.

Demonstração. Pela Proposição D.3, basta mostrar que para $\theta \in \mathbb{R}^{m}$,

$$
\mathbb{E}\left[\exp \left(\imath \sum_{j=1}^{m} \theta_{j} W_{t_{j}}\right)\right]=\exp \left(-\frac{1}{2}\left(\sum_{j=1}^{m} \sum_{k=1}^{m} \theta_{j} \theta_{k}\left(t_{j} \wedge t_{k}\right)\right)\right) .
$$

De fato, seja $0 \leq t_{1}<t_{2} \ldots<t_{m}<1$ uma sequência arbitrária e considere o vetor $\left(W_{t_{1}}^{n}, W_{t_{2}}^{n}, \ldots, W_{t_{m}}^{n}\right)$. Calculando a função característica, temos

$$
\begin{aligned}
\mathbb{E}\left[\exp \left(\imath \sum_{j=1}^{m} \theta_{j} W_{t_{j}}^{n}\right)\right] & =\mathbb{E}\left[\exp \left(\imath \sum_{j=1}^{m} \theta_{j} \sum_{i=1}^{n} X_{i} \int_{0}^{t_{j}} \psi_{i}(\xi) \lambda(d \xi)\right)\right] \\
& =\mathbb{E}\left[\prod_{i=1}^{n} \exp \left(\imath \sum_{j=1}^{m} \theta_{j} X_{i} \int_{0}^{t_{j}} \psi_{i}(\xi) \lambda(d \xi)\right)\right] \\
& =\prod_{i=1}^{n} \mathbb{E}\left[\exp \left(\imath \sum_{j=1}^{m} \theta_{j} \int_{0}^{t_{j}} \psi_{i}(\xi) \lambda(d \xi) X_{i}\right)\right]
\end{aligned}
$$

Mas, $\mathbb{E}\left[\exp \left(\imath \sum_{j=1}^{m} \theta_{j} \int_{0}^{t_{j}} \psi_{i}(\xi) \lambda(d \xi) X_{i}\right)\right]$ é a função característica de uma variável aleatória Normal, com média 0 e variância 1 , calculada em $t=\sum_{j=1}^{m} \theta_{j} \int_{0}^{t_{j}} \psi_{i}(\xi) \lambda(d \xi)$. Assim, pela Proposição D.3 temos que

$$
\mathbb{E}\left[\exp \left(\imath \sum_{j=1}^{m} \theta_{j} \int_{0}^{t_{j}} \psi_{i}(\xi) \lambda(d \xi) X_{i}\right)\right]=\exp \left(-\frac{1}{2}\left(\sum_{j=1}^{m} \theta_{j} \int_{0}^{t_{j}} \psi_{i}(\xi) \lambda(d \xi)\right)^{2} 1^{2}\right) .
$$


Disso e de (D.9), ficamos com

$$
\begin{aligned}
\mathbb{E}\left[\exp \left(\imath \sum_{j=1}^{m} \theta_{j} W_{t_{j}}^{n}\right)\right] & =\prod_{i=1}^{n} \exp \left(-\frac{1}{2}\left(\sum_{j=1}^{m} \theta_{j} \int_{0}^{t_{j}} \psi_{i}(\xi) \lambda(d \xi)\right)^{2}\right) \\
& =\exp \left(-\frac{1}{2} \sum_{i=1}^{n}\left(\sum_{j=1}^{m} \theta_{j} \int_{0}^{t_{j}} \psi_{i}(\xi) \lambda(d \xi)\right)^{2}\right) \\
& =\exp \left(-\frac{1}{2} \sum_{i=1}^{n}\left(\sum_{j=1}^{m} \sum_{k=1}^{m} \theta_{j} \theta_{k} \int_{0}^{t_{j}} \psi_{i}(\xi) \lambda(d \xi) \int_{0}^{t_{k}} \psi_{i}(\xi) \lambda(d \xi)\right)\right) \\
& =\exp \left(-\frac{1}{2}\left(\sum_{j=1}^{m} \sum_{k=1}^{m} \theta_{j} \theta_{k} \sum_{i=1}^{n}<I_{t_{j}}, \psi_{i}><I_{t_{k}}, \psi_{i}>\right)\right)
\end{aligned}
$$

Mas, pelo Teorema D.13, $W_{t}^{n} \rightarrow W_{t}$ em $\mathrm{L}^{2}(\Omega, \mathscr{F}, \mathrm{P})$. Isso implica que existe uma subsequência $\left\{W_{t}^{n_{l}}\right\}$ tal que $W_{t}^{n_{l}} \rightarrow W_{t}$ q. s., quando $l \rightarrow \infty$. Assim, pelo Teorema da convergência limitada (veja, por exemplo, [1] página 210), temos que

$$
\mathbb{E}\left[\exp \left(\imath \sum_{j=1}^{m} \theta_{j} W_{t_{j}}^{n}\right)\right] \rightarrow \mathbb{E}\left[\exp \left(\imath \sum_{j=1}^{m} \theta_{j} W_{t_{j}}\right)\right]
$$

e portanto, por (D.10)

$$
\begin{aligned}
\mathbb{E}\left[\exp \left(\imath \sum_{j=1}^{m} \theta_{j} W_{t_{j}}\right)\right] & =\lim _{n \rightarrow \infty} \exp \left(-\frac{1}{2}\left(\sum_{j=1}^{m} \sum_{k=1}^{m} \theta_{j} \theta_{k} \sum_{i=1}^{n}<I_{t_{j}}, \psi_{i}><I_{t_{k}}, \psi_{i}>\right)\right) \\
& =\exp \left(-\frac{1}{2}\left(\sum_{j=1}^{m} \sum_{k=1}^{m} \theta_{j} \theta_{k}<I_{t_{j}}, I_{t_{k}}>\right)\right) \\
& =\exp \left(-\frac{1}{2}\left(\sum_{j=1}^{m} \sum_{k=1}^{m} \theta_{j} \theta_{k}\left(t_{j} \wedge t_{k}\right)\right)\right)
\end{aligned}
$$

Como queríamos. 
Assim, os três últimos resultados, garantem que o processo $\left\{W_{t}: t \in[0,1]\right\}$ definido por

$$
W_{t}=\lim _{n \rightarrow \infty} \sum_{i=1}^{n} X_{i} \int_{0}^{t} \psi_{i}(\xi) \lambda(d \xi)
$$

satisfaz as condições do Teorema D.9, e portanto $\left\{W_{t}: t \in[0,1]\right\}$ satifaz as três primeiras condições para ser um movimento Browniano. Para que satisfaça a última, devemos fazer uma boa escolha da base $\left\{\psi_{i}\right\}$. É disso o que vamos tratar agora.

\section{D.4.2 A última propriedade}

\section{Uma base para $L^{2}[0,1]$ - Funções de Haar e de Schauder}

As funções de Haar em $[0,1]$ são definidas da seguinte maneira: $f_{0}(t) \equiv 1$ e para cada $n=1,2, \ldots$, $j=1, \ldots, 2^{n-1}, f_{j, n}$ é definido como

$$
f_{j, n}(t)=\left\{\begin{array}{l}
2^{(n-1) / 2}, \frac{k-1}{2^{n}} \leq t \leq \frac{k}{2^{n}} \\
-2^{(n-1) / 2}, \frac{k}{2^{n}}<t \leq \frac{k+1}{2^{n}} \\
0, \text { caso contrário }
\end{array}\right.
$$

onde $k=2 j-1$.

Teorema D.15. As funções de Haar formam uma base ortonormal completa para $\mathfrak{H}$.

Demonstração. É fácil ver que as funções são ortogonais e tem norma 1. Para ver que a base é completa, note que $\mathbb{1}_{[0,1 / 2)}$ e $\mathbb{1}_{[1 / 2,1)}$ são ambas combinações lineares de $f_{0}$ e $f_{1,1}$, assim como $\mathbb{1}_{[0,1 / 4)}$ e $\mathbb{1}_{[1 / 4,2 / 4)}$ são ambas combinações lineares de $\mathbb{1}_{[0,1 / 2)}, f_{1,2}$ e $f_{2,2}$. Continuando desta maneira, temos que $\mathbb{1}_{\left[k / 2^{n},(k+1) / 2^{n}\right.}$ é uma combinação linear dos $f_{j, n}$ para cada $n$ e cada $k \leq 2^{n}$. Como qualquer função contínua pode ser aproximada uniformemente por funções simples com saltos nos racionais diádicos, o conjunto das combinações lineares das funções de Haar é densa no conjunto de funções contínuas. Porém, como o conjunto das funções contínuas é denso em $\mathfrak{H}$ segue o que queríamos.

As funções de Schauder, $\left\{F_{0}, F_{n, j}\right\}$ são as integrais indefinidas das funções de Haar, ou seja, 
$F_{0}(t)=t \mathrm{e}$

$$
F_{j, n}(t)=\left\{\begin{array}{l}
2^{(n-1) / 2}\left(t-(k-1) / 2^{n}\right), \frac{k-1}{2^{n}} \leq t<\frac{k}{2^{n}} \\
-2^{(n-1) / 2}\left((k+1) / 2^{n}-t\right), \frac{k}{2^{n}} \leq t<\frac{k+1}{2^{n}} \\
0, \text { caso contrário }
\end{array}\right.
$$

onde $k=2 j-1$. As funções de Schauder, são como pequenas "tendas" de altura $2^{-(n+1) / 2}$, como ilustrado na figura abaixo.

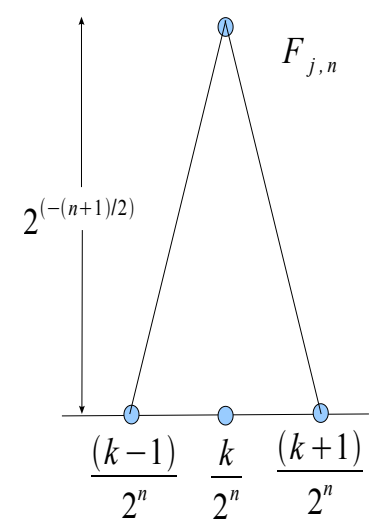

Note também, que segue pela definição das funções de Schauder, que para cada $n$ fixo, $F_{1, n}, F_{2, n}, \ldots$ são não nulas em intervalos disjuntos. Para mostrar que podemos construir um processo satisfazendo a propriedade (B4), vamos utilizar um conhecido resultado da análise real

Proposição D.16. Seja $\left\{f_{n}\right\}$ uma sequência de funções contínuas definidas em $[0,1]$ tais que $f_{n}$ converge uniformemente para uma função $f$. Então $f$ é uma função contínua.

Agora seja $\left\{X_{0}, X_{j, n}, n=1,2, \ldots, j=1,2, \ldots, 2^{n-1}\right\}$ uma sequência de variáveis aleatórias normais com média 0 e variância 1 e independentes. Para cada $t \in[0,1]$ e $N=1,2, \ldots$ defina

$$
W_{t}^{N}(\omega) \doteq X_{0}(\omega) F_{0}(t)+\sum_{n=1}^{N} Y_{n}(t, \omega)
$$

onde

$$
Y_{n}(t, \omega) \doteq \sum_{j=1}^{2^{n-1}} X_{j, n} \int_{0}^{t} f_{j, n}(s) d t=\sum_{j=1}^{2^{n-1}} X_{j, n} F_{j, n}(t)
$$


Teorema D.17. A sequência $W_{t}^{N}$ definida por (D.11) converge uniformemente em $t$, q. s.. Portanto, o processo $W_{t}=\lim _{N \rightarrow \infty} W_{t}^{N}$ é um processo estocástico com trajétorias contínuas.

Demonstração. De fato, se provarmos a convergência uniforme, então o resultado segue por conta da Proposição D.16. Agora, seja $H_{n}(\omega)=\max _{t \in[0,1]}\left|Y_{n}(t, \omega)\right|$. Como, para $n$ fixado, o suporte das funções de Schauder $F_{n, 1}, F_{n, 2}, \ldots$ é disjunto e o valor máximo que ela assume é $2^{-(n+1) / 2}$ temos que $H_{n}=2^{-(n+1) / 2} \max _{1 \leq j \leq 2^{n-1}}\left|X_{j, n}\right|$. Seja $c_{n}$ uma constante qualquer, temos

$$
\begin{aligned}
\mathrm{P}\left[H_{n}>2^{-(n+1) / 2} c_{n}\right] & =\mathrm{P}\left[\max _{1 \leq j \leq 2^{n-1}} \mid X_{j, n}>c_{n}\right] \\
& =\mathrm{P}\left[\bigcup_{j=1}^{2^{n-1}}\left[\left|X_{j, n}\right|>c_{n}\right]\right] \\
& \leq \sum_{j=1}^{2^{n-1}} \mathrm{P}\left[\left|X_{j, n}\right|>c_{n}\right] .
\end{aligned}
$$

pelo Lema D.6 e por (D.12) resulta que

$$
\mathrm{P}\left[H_{n}>2^{-(n+1) / 2} c_{n}\right] \leq 2^{n-1} \frac{2 \exp \left(-\frac{1}{2} c_{n}^{2}\right)}{c_{n} \sqrt{2 \pi}} .
$$

Agora, tome $c_{n}=\theta \sqrt{2 n \log (2)}$ para algum $\theta>1$. Assim (D.13) fica

$$
\mathrm{P}\left[H_{n}>\theta \sqrt{n 2^{-n} \log (2)}\right] \leq K \frac{2^{n\left(1-\theta^{2}\right)}}{\sqrt{n}}
$$

Assim, como $\sum_{n} \mathrm{P}\left[H_{n}>\theta \sqrt{n 2^{-n} \log (2)}\right]<\sum_{n} K \frac{2^{n\left(1-\theta^{2}\right)}}{\sqrt{n}}<\infty$ o lema de Borel-Cantelli garante que

$$
\mathrm{P}\left[\lim \sup \left\{H_{n}>\theta \sqrt{n 2^{-n} \log (2)}\right\}\right]=0
$$

ou seja, para quase todo $\omega$, existe $N(\omega)$ tal que para $n \geq N(\omega)$

$$
H_{n}(\omega) \leq \theta \sqrt{n 2^{-n} \log (2)}
$$


mas como $\sum_{n} \theta \sqrt{n 2^{-n} \log (2)}<\infty$, temos que $\sum_{n} H_{n}<\infty$ e portanto

$$
\begin{aligned}
\lim _{N \rightarrow \infty}\left|W_{t}^{N}(\omega)\right| & \leq X_{0}(\omega) F_{0}(t)+\sum_{n=1}^{\infty}\left|Y_{n}(t, \omega)\right| \\
& \leq X_{0}(\omega) F_{0}(t)+\sum_{n=1}^{\infty} H_{n}(\omega) \\
& \leq X_{0}(\omega) F_{0}(t)+\sum_{n=1}^{\infty} \theta \sqrt{n 2^{-n} \log (2)} \\
<\infty &
\end{aligned}
$$

Ou seja, $\left\{W_{t}^{N}(\omega)\right\}$ converge uniformemente, $\mathrm{P}-\mathrm{q}$. s..

Assim, pelos Teoremas D.14 e D.17, provamos

Teorema D.18. Existe um movimento Browniano em $[0,1]$.

\section{D.5 Extensão para $[0, \infty)$}

Antes provar o resultado principal, lembramos que dois processos $\left\{X_{t}: t \in[0, T)\right\}$ e $\left\{Y_{t}: t \in\right.$ $[0, T)\}$ são ditos independentes, se as $\sigma$-álgebras $\sigma\left(\left\{X_{t}: t \in[0, T)\right\}\right)$ e $\sigma\left(\left\{Y_{t}: t \in[0, T)\right\}\right)$ são independentes. Em particular, isso implica que para quaisquer $0 \leq \widetilde{t}, \widehat{t}<T$, as váriaveis aleatórias $X_{\widetilde{t}} \mathrm{e}$ $Y_{\widehat{t}}$ são independentes, pois $\sigma\left(\left\{X_{\tilde{t}}\right\}\right) \subseteq \sigma\left(\left\{X_{t}: t \in[0, T)\right\}\right)$ e $\sigma\left(\left\{Y_{\widehat{t}}\right\}\right) \subseteq \sigma\left(\left\{Y_{t}: t \in[0, T)\right\}\right)$, e portanto $\sigma\left(\left\{X_{\widetilde{t}}\right\}\right)$ é independente de $\sigma\left(\left\{Y_{\widehat{t}}\right\}\right)$.

Teorema D.19. Existe um processo $\left\{B_{t}: t \in(0,+\infty]\right\}$, satisfazendo $(\mathrm{B} 1)-(\mathrm{B} 4)$.

Demonstração. Em primeiro lugar, pelo Teorema D.18 e pelo Teorema de existência de Kolmovogorv (veja, por exemplo, [1]), podemos construir uma seqûencia $B_{t}^{(0)}, B_{t}^{(1)}, \ldots$ de movimentos Brownianos independentes em $[0,1]$. Considere o processo $\left\{W_{t}: t \in[0, \infty)\right\}$ definido por

$$
W_{t}=\left\{\begin{array}{l}
B_{t}^{(0)}, \text { se } t \in[0,1] \\
\sum_{k=0}^{j-1} B_{1}^{(k)}+B_{t-j}^{(j)}, \text { se } t \in[j, j+1], j \geq 1
\end{array}\right.
$$

O processo satisfaz a propriedade (B4) pois é a soma de funções contínuas q. s.. Para ver que as outras propriedades são satisfeitas, vamos verificar que $\left\{W_{t}\right\}$ satisfaz as hipóteses do Corolário D.10. 
Temos que $\left\{W_{t}\right\}$ é um processo Gaussiano, pois para uma sequência arbitrária $0 \leq t_{1}<t_{2}<\ldots<t_{n}$, qualquer combinação linear de $W_{t_{1}}, W_{t_{2}}, \ldots$ pode ser escrita como

$$
\sum_{i=1}^{n_{0}} a_{i, 0} B_{t_{i, 0}}^{(0)}+\sum_{i=1}^{n_{1}} a_{i, 1} B_{t_{i, 1}}^{(1)}+\cdots+\sum_{i=1}^{n_{k}} a_{i, k} B_{t_{i, k}}^{(k)}
$$

e cada parcela acima é Normal (pois cada $B^{(i)}$ é Gaussiano), e a soma das parcelas também é pois por hipótese, a sequência $B_{t}^{(0)}, B_{t}^{(1)}, \ldots$ é de movimentos Brownianos independentes, e a soma de variavéis aleatórias normais independentes é Normal. Além disso, $\mathbb{E}\left[W_{t}\right]=0$ para qualquer $t \in[0, \infty)$ pois $W_{t}$ é a soma finita de variavéis aleatórias com média 0 . Finalmente, seja $s \leq t$. Temos duas possibilidades. Ou existe $j \geq 0$ tal que $j \leq s \leq t<t+1$ ou $j \leq s \leq j+1 \leq l \leq t<l+1$ para algum $l>j$. No primeiro caso,

$$
\begin{aligned}
\operatorname{Cov}\left(W_{s}, W_{t}\right) & =\mathbb{E}\left[W_{s} W_{t}\right] \\
& =\mathbb{E}\left[\left(\sum_{k=0}^{j-1} B_{1}^{(k)}+B_{t-j}^{(j)}\right)\left(\sum_{k=0}^{j-1} B_{1}^{(k)}+B_{s-j}^{(j)}\right)\right] \\
& =\mathbb{E}\left[\sum_{k=0}^{j-1}\left(B_{1}^{(k)}\right)^{2}\right]+\mathbb{E}\left[B_{s-j}^{(j)} B_{t-j}^{(j)}\right]+ \\
& +\left(\sum_{k=0}^{j-1} \mathbb{E}\left[B_{1}^{(k)} B_{s-j}^{(j)}\right]+\sum_{k=0}^{j-1} \mathbb{E}\left[B_{1}^{(k)} B_{t-j}^{(j)}\right]+2 \sum_{k \neq l}^{j-1} \mathbb{E}\left[B_{1}^{(k)} B_{1}^{(l)}\right]\right)
\end{aligned}
$$

Note que a última parcela entre parênteses é nula pois são somas de esperanças do produto de variáveis aleatórias independentes de média 0 . Ficamos portanto com

$$
\begin{aligned}
\operatorname{Cov}\left(W_{s}, W_{t}\right) & =\mathbb{E}\left[\sum_{k=0}^{j-1}\left(B_{1}^{(k)}\right)^{2}\right]+\mathbb{E}\left[B_{s-j}^{(j)} B_{t-j}^{(j)}\right] \\
& =j \times 1+(s-j) \\
& =s \\
& =s \wedge t
\end{aligned}
$$


No segundo caso, temos

$$
\begin{aligned}
\operatorname{Cov}\left(W_{s}, W_{t}\right) & =\mathbb{E}\left[W_{s} W_{t}\right] \\
& =\mathbb{E}\left[\left(\sum_{k=0}^{j-1} B_{1}^{(k)}+B_{s-j}^{(j)}\right)\left(\sum_{k=0}^{j-1} B_{1}^{(k)}+B_{1}^{(j)}+\sum_{k=j+1}^{l-1} B_{1}^{(k)}+B_{t-l}^{(l)}\right)\right] \\
& =\mathbb{E}\left[\sum_{k=0}^{j-1}\left(B_{1}^{(k)}\right)^{2}\right]+\mathbb{E}\left[B_{s-j}^{(j)} B_{1}^{(j)}\right]+A
\end{aligned}
$$

Aqui $A$, como no caso anterior, é uma parcela composta de somas de esperanças do produto de variáveis aleatórias independentes de média 0 . Além disso, como $s-j \leq 1$, ficamos com

$$
\begin{aligned}
\operatorname{Cov}\left(W_{s}, W_{t}\right) & =\mathbb{E}\left[\sum_{k=0}^{j-1}\left(B_{1}^{(k)}\right)^{2}\right]+(s-j) \\
& =j+(s-j) \\
& =s \\
& =s \wedge t
\end{aligned}
$$

E está provado o que queríamos.

Existem outras maneiras extender o movimento Browniano para $[0, \infty]$ dada a existência de um em $[0,1]$. Para o leitor interessado, recomendamos [2]. 


\section{Apêndice E}

\section{Teorema de Girsanov}

Practical men, who believe themselves to be quite exempt from any intellectual influence, are usually the slaves of some defunct economist.

J. M. Keynes

Nesse apendice desenvolveremos a teoria de mudança de medida, culminando no teorema de Girsanov. Os resultados obtidos aqui são muito utlizados para aplicações em finanças. Mesmo que não seja o foco deste texto apresentar tais aplicações, achamos conveniente incluir este apêndice como aplicação da teoria desenvolvida neste trabalho.

\section{E.1 Teorema de Bayes}

Começaremos lembrando o leitor que dadas duas medidas de probabilidade $\mathrm{P}$ e Q, dizemos que $\mathrm{Q}$ é absolutamente contínua com respeito a $\mathrm{P}$ (e a $\sigma$-álgebra $\mathscr{F}$ ) e denotamos $\mathrm{Q} \ll \mathrm{P}$, quando para todo $F \in \mathscr{F}$,

$$
\mathrm{P}(F)=0 \Rightarrow \mathrm{Q}(F)=0
$$

Com a introdução do próximo conceito, poderemos nos referir a resultados valendo quase certamente sem precisar especificar a medida de probabilidade que estamos usando.

Definição E.1. Dizemos que duas medidas de probabilidade $\mathrm{P}$ e $\mathrm{Q}$ são equivalentes quando $\mathrm{P} \ll \mathrm{Q}$ $e \mathrm{Q} \ll \mathrm{P}$. Denotamos essa condição usando a notação $\mathrm{P} \sim \mathrm{Q}$. Além disso, dizemos que $\mathrm{P}$ e $\mathrm{Q}$ são $\left\{\mathscr{F}_{t}\right\}$-localmente equivalentes quando as medidas $\mathrm{P}_{t} \doteq \mathrm{P} \mid \mathscr{F}_{t}$ e $\mathrm{Q}_{t} \doteq \mathrm{Q} \mid \mathscr{F}_{t}$ forem equivalentes para 
cada $t \geq 0$.

Veremos adiante um exemplo mostrando que medidas localmente equivalentes não são, necessariamente, equivalentes em $\mathscr{F}_{\infty}$.

Lema E.2. Sejam P, Q medidas de probabilidades $\left\{\mathscr{F}_{t}\right\}$-localmente equivalentes, $M_{t}$ a derivada de Radon-Nikodym de $\mathrm{Q}_{t}$ com respeito a $\mathrm{P}_{t}$ e $f$ uma função $\mathscr{F}_{t}$-mensurável. Então $f \in \mathrm{L}^{1}(\mathrm{Q})$ se, e somente se, $M_{t} f \in \mathrm{L}^{1}(\mathrm{P})$ e nesse caso $\mathbf{E}_{\mathrm{Q}}(f)=\mathbf{E}_{\mathrm{P}}\left(M_{t} f\right)$.

Demonstração. De fato, para cada $t \geq 0$ e $A \in \mathscr{F}_{t}$, temos pelo teorema de Radon-Nikodym* que

$$
\mathrm{Q}(A)=\mathrm{Q}_{t}(A)=\mathbf{E}_{\mathrm{Q}_{t}}\left(\mathbb{1}_{[A]}\right)=\mathbf{E}_{\mathrm{P}_{t}}\left(M_{t} \mathbb{1}_{[A]}\right)=\mathbf{E}_{\mathrm{P}}\left(M_{t} \mathbb{1}_{[A]}\right)
$$

Não é difícil verificar que estamos sob as condições do teorema da extensão e portanto a relação

$$
\mathbf{E}_{\mathrm{Q}}(f)=\mathbf{E}_{\mathrm{Q}_{t}}(f)=\mathbf{E}_{\mathrm{P}_{t}}\left(M_{t} f\right)=\mathbf{E}_{\mathrm{P}}\left(M_{t} f\right)
$$

é válida para qualquer função não negativa $f \mathscr{F}{ }$-mensurável. O resultado segue usando a decomposição $f=f^{+}-f^{-}$e a linearidade da esperança.

O próximo teorema generaliza esses resultados para esperanças condicionais.

Teorema E.3 (Teorema de Bayes). Sejam $\mathrm{P}$ e Q duas medidas de probabilidade localmente equivalentes. Então $M_{t}=\frac{d \mathrm{Q}_{t}}{d \mathrm{P}_{t}}$ é um martingal estritamente positivo com respeito a medida $\mathrm{P}$ e para cada $0 \leq t<T$ e $f \in \mathrm{L}^{1}\left(\mathrm{Q}, \mathscr{F}_{T}\right)$ temos que

$$
\mathbf{E}_{\mathrm{Q}}\left(f \| \mathscr{F}_{t}\right)=\frac{\mathbf{E}_{\mathrm{P}}\left(M_{T} f \| \mathscr{F}_{t}\right)}{M_{t}}=\frac{\mathbf{E}_{\mathrm{P}}\left(M_{T} f \| \mathscr{F}_{t}\right)}{\mathbf{E}_{\mathrm{P}}\left(M_{T} \| \mathscr{F}_{t}\right)} .
$$

Demonstração. Fixe $0 \leq t<T$. Como $\mathrm{P}_{t}$ e $\mathrm{Q}_{t}$ são equivalentes com respeito a $\mathscr{F}_{t}$, do teorema de Radon-Nikodym temos que $M_{t}$ é estritamente positiva P-q. s. e $M_{t} \in \mathrm{L}^{1}\left(\mathrm{P}, \mathscr{F}_{t}\right)$. Em particular, $M_{t}$ é $\mathscr{F}_{t}$-mensurável. Agora seja $A \in \mathscr{F}_{t} \subseteq \mathscr{F}_{T}$. Pelo lema anterior, temos que $\mathrm{Q}(A)=\mathbf{E}_{\mathrm{P}}\left(M_{t} \mathbb{1}_{[A]}\right)$ e também $\mathrm{Q}(A)=\mathbf{E}_{\mathrm{Q}}\left(M_{T} \mathbb{1}_{[A]}\right)$. Disso resulta que $\mathbf{E}_{\mathrm{P}}\left(M_{t} \mathbb{1}_{[A]}\right)=\mathbf{E}_{\mathrm{Q}}\left(M_{T} \mathbb{1}_{[A]}\right)$ e portanto, pelo teorema C.8 na página 159 , resulta que $M_{t}=\mathbf{E}_{\mathrm{P}}\left(M_{T} \| \mathscr{F}_{t}\right)$.

\footnotetext{
* Veja o teorema C.5 na página 158. † Veja o teorema A.3 na página 152.
} 
Vamos provar agora que $M_{t} \mathbf{E}_{\mathrm{Q}}\left(f \| \mathscr{F}_{t}\right)=\mathbf{E}_{\mathrm{P}}\left(M_{T} f \| \mathscr{F}_{t}\right)$. Seja $A \in \mathscr{F}_{t}$. Temos pela definição de esperança condicional que $\mathbf{E}_{\mathrm{Q}}\left(f \| \mathscr{F}_{t}\right)$ é $\mathscr{F}_{t}$-mensurável. Agora, pela propriedade da torre* temos que

$$
\mathbf{E}_{\mathrm{Q}}\left(\mathbf{E}_{\mathrm{Q}}\left(f \| \mathscr{F}_{t}\right) \mathbb{1}_{[A]}\right)=\mathbf{E}_{\mathrm{Q}}\left(\mathbf{E}_{\mathrm{Q}}\left(f \mathbb{1}_{[A]} \| \mathscr{F}_{t}\right)\right)=\mathbf{E}_{\mathrm{Q}}\left(f \mathbb{1}_{[A]}\right)
$$

Agora, como $\mathbf{E}_{\mathrm{Q}}\left(f \| \mathscr{F}_{t}\right) \mathbb{1}_{[A]}$ é $\mathscr{F}_{t}$-mensurável e $f \mathbb{1}_{[A]}$ é $\mathscr{F}_{T}$-mensurável, por (E.1) temos que

$$
\mathbf{E}_{\mathrm{Q}}\left(\mathbf{E}_{\mathrm{Q}}\left(f \| \mathscr{F}_{t}\right) \mathbb{1}_{[A]}\right)=\mathbf{E}_{\mathrm{P}}\left(M_{t} \mathbf{E}_{\mathrm{Q}}\left(f \| \mathscr{F}_{t}\right) \mathbb{1}_{[A]}\right)
$$

$\mathrm{e}$

$$
\mathbf{E}_{\mathrm{Q}}\left(f \mathbb{1}_{[A]}\right)=\mathbf{E}_{\mathrm{P}}\left(M_{T} f \mathbb{1}_{[A]}\right)
$$

e assim de (E.2), (E.3) e (E.4) temos que

$$
\mathbf{E}_{\mathrm{P}}\left(M_{t} \mathbf{E}_{\mathrm{Q}}\left(f \| \mathscr{F}_{t}\right) \mathbb{1}_{[A]}\right)=\mathbf{E}_{\mathrm{P}}\left(M_{T} f \mathbb{1}_{[A]}\right)
$$

Como $M_{t} \mathbf{E}_{Q}\left(f \| \mathscr{F}_{t}\right)$ é $\mathscr{F}_{t}$-mensurável, do teorema C.8 na página 159 , resulta que

$$
M_{t} \mathbf{E}_{\mathrm{Q}}\left(f \| \mathscr{F}_{t}\right)=\mathbf{E}_{\mathrm{P}}\left(M_{T} f \| \mathscr{F}_{t}\right)
$$

como queríamos.

Uma consequência deste teorema, importante para as aplicações, é o próximo

Corolário E.4. Seja $\mathbf{M}$ a derivada de Radon-Nikodym de Q com respeito a P. Um processo estocástico adaptado $\mathbf{X}$ é um martingal local com respeito a $\mathrm{Q}$ se e somente se o processo (XM) for um martingal local com respeito a $\mathrm{P}$.

Demonstração. Primeiramente, vamos provar o resultado para martingais. De fato, Seja $\mathbf{X}$ um martingal com respeito a Q . Então $X_{t}$ é $\mathscr{F}_{t}$-mensurável e $X_{t} \in \mathrm{L}^{1}(\mathrm{Q})$. Pelo lema E.2, segue que $X_{t} M_{t} \in \mathrm{L}^{1}(\mathrm{P})$ e é obviamente adaptado. Agora, temos também que $X_{T} \in \mathrm{L}^{1}(\mathrm{Q})$ e pelo teorema anterior segue que

$$
M_{t} \mathbf{E}_{\mathrm{Q}}\left(X_{T} \| \mathscr{F}_{t}\right)=M_{t} X_{t}=\mathbf{E}_{\mathrm{P}}\left(M_{T} X_{T} \| \mathscr{F}_{t}\right)
$$

isso mostra que $\mathbf{M X}$ é um martingal com respeito a $\mathrm{P}$. Reciprocamente, sendo $\mathbf{M X}$ um martingal com respeito a $\mathrm{P}$, temos que $M_{t} X_{t} \in \mathrm{L}^{1}(\mathrm{P})$ e portanto pelo lema E.2, $X_{t} \in \mathrm{L}^{1}(\mathrm{Q})$. Além disso,

\footnotetext{
* Veja o teorema C.11 na página 161.
} 
da relação $M_{t} X_{t}=\mathbf{E}_{\mathrm{P}}\left(M_{T} X_{T} \| \mathscr{F}_{t}\right)$ resulta pelo teorema anterior que $X_{t}=\mathbf{E}_{\mathrm{Q}}\left(X_{T} \| \mathscr{F}_{t}\right)$ para todo $t \in[0, T]$.

Vamos agora provar o resultado para martingais locais. Assim, suponha que $\mathbf{X}$ é um martingal local com respeito a $\mathrm{Q}$ e seja $\left\{\tau_{n}\right\}_{n \geq 1}$ uma sequência de tempos de parada tal que $\tau_{n} \uparrow \infty$ para todo $\omega \in \Omega^{*}$. Temos que $\mathbf{X}^{\tau_{n}} \mathbb{1}_{\left[\tau_{n}>0\right]}$ é um Q-martingal (indexado por $t$ ) e portanto pelo o que provamos acima, $\mathbf{Y}=\mathbf{M X}^{\tau_{n}} \mathbb{1}_{\left[\tau_{n}>0\right]}$ é um P-martingal. Como martingal parado também é martingal ${ }^{\dagger}$, $\mathbf{Y}^{\tau_{n}}=\mathbf{X}^{\tau_{n}} \mathbb{1}_{\left[\tau_{n}>0\right]} \mathbf{M}^{\tau_{n}}$ também é um P-martingal para cada $n \geq 1$. Dessa forma, temos que $\left\{\tau_{n}\right\}_{n \geq 1}$ é uma sequência redutora para $\mathbf{M X}$ e portanto provamos que $\mathbf{M X}$ é um P-martingal local. A recíproca segue trocando $\mathrm{P}$ e Q e usando o fato que $\frac{d \mathrm{P}_{t}}{d \mathrm{Q}_{t}}=M_{t}^{-1}$.

\section{E.2 Mudança de medida}

Sejam P, Q medidas localmente equivalentes com respeito a filtração $\left\{\mathscr{F}_{t}\right\}$. Pelo teorema de Radon-Nikodym e pelos resultados da seção anterior, temos que o processo $\mathbf{M}$ definido para cada $t \geq 0$ como

$$
M_{t}=\frac{d \mathrm{Q}_{t}}{d \mathrm{P}_{t}},
$$

onde $\mathrm{Q}_{t}$ é a restrição da medida $\mathrm{Q}$ em $\mathscr{F}_{t}$ e $\mathrm{P}_{t}$ é a restrição da medida $\mathrm{P}$ em $\mathscr{F}_{t}$ é um P-martingal estritamente positivo com $M_{0}=1$. Chamaremos $\left\{\frac{d \mathrm{Q}_{t}}{d \mathrm{P}_{t}}\right\}_{t \geq 0}$ de processo densidade associado às medidas $\mathrm{P}$ e $\mathrm{Q}$.

Vamos assumir que o processo $\mathbf{M}$ possui uma versão contínua. Lembre-se que pelo teorema 2.29 na página 19, M possui uma versão càdlàg. É possível provar (veja [23]) que se a filtração $\left\{\mathscr{F}_{t}\right\}$ for a aumentada e gerada por um movimento Browniano então ela é contínua à direita e assim, todo martingal adaptado a esta filtração admitirá uma versão contínua.

\section{E.2.1 A fórmula de Girsanov}

O objetivo agora será provar a fórmula de Girsanov. Ela mostra quando um semi-martingal com respeito a uma medida pode ser um semi-martingal com respeito a outra e apresenta a fórmula do compensador do semi-martingal na nova medida. Começaremos antes com um lema simples.

Lema E.5. Sejam $\mathrm{P}$ e Q medidas localmente equivalentes com respeito a filtração $\left\{\mathscr{F}_{t}\right\}$. Então A é um processo contínuo, de variação finita com respeito a medida $\mathrm{P}$ se, e somente se, for um processo

\footnotetext{
* Veja a proposição 2.50 na página $29 . \quad$ † Veja o corolário 2.38 na página 22.
} 
contínuo, de variação finita com respeito a medida $\mathrm{Q}$.

Demonstração. Seja $\Lambda \subseteq \Omega$ o conjunto de todos os $\omega \in \Omega$ tal que as trajetórias $t \mapsto A(t, \omega)$ são contínuas e de variação finita em todos os intervalos finitos. Temos que $\Lambda=\cap_{n} \Lambda_{n}$, onde $\Lambda_{n}$ é o conjunto de todos os $\omega \in \Omega$ tal que as trajetórias são contínuas e de variação finita no intervalo $[0, n]$. Para cada $n \geq 1$, temos que $\Lambda_{n} \in \mathscr{F}_{n}$ e como $\mathrm{P}$ e Q são localmente equivalentes, temos que $\mathrm{P}\left(\Lambda_{n}\right)=1$ se, e somente se, $\mathrm{Q}\left(\Lambda_{n}\right)=1$. Disso resulta que $\mathrm{P}(\Lambda)=1$ se, e somente se, $\mathrm{Q}(\Lambda)=1$. Como queríamos.

Proposição E.6 (Fórmula de Girsanov). Sejam P, Q medidas localmente equivalentes com respeito a filtração $\left\{\mathscr{F}_{t}\right\}, \mathbf{M}$ o processo densidade e $\mathbf{X}$ um $\mathrm{P}$-semi-martingal contínuo. Então $\mathbf{X}$ é um $\mathrm{Q}$ semi-martingal contínuo e o seu compensador com respeito à medida $\mathrm{Q}$ é $u_{\mathbf{X}}^{\mathrm{Q}}=u_{\mathbf{X}}^{\mathrm{P}}+\llbracket \mathbf{X}, \log (\mathbf{M}) \rrbracket$.

Demonstração. Lembre-se que pela definição de semi-martingal, X é um Q-semi-martingal se, e somente se, existe um processo $\mathbf{A}$ contínuo, de variação finita e nulo em $t=0$ tal que $\mathbf{X}-\mathbf{A}$ seja um Q-martingal local (contínuo). Mas pelo corolário E.4, X - A é um Q-martingal local se, e somente se, $(\mathbf{X}-\mathbf{A}) \mathbf{M}$ for um P-martingal local. Porém $(\mathbf{X}-\mathbf{A}) \mathbf{M}$ é um P-martingal local se, e somente se, $u_{(\mathbf{X}-\mathbf{A}) \mathbf{M}}^{\mathrm{P}}=0$, equivalentemente,

$$
d u_{(\mathbf{X}-\mathbf{A}) \mathbf{M}}^{\mathrm{P}}=0
$$

Agora pela proposição 4.50 na página 115, item iv)

$$
\begin{aligned}
0=d u_{(\mathbf{X}-\mathbf{A}) \mathbf{M}}^{\mathrm{P}} & =(\mathbf{X}-\mathbf{A}) d u_{\mathbf{M}}^{\mathrm{P}}+\mathbf{M} d u_{\mathbf{X}-\mathbf{A}}^{\mathrm{P}}+d \llbracket \mathbf{X}-\mathbf{A}, \mathbf{M} \rrbracket \\
& =\mathbf{M} d u_{\mathbf{X}}^{\mathrm{P}}-\mathbf{M} d \mathbf{A}+d \llbracket \mathbf{X}, \mathbf{M} \rrbracket
\end{aligned}
$$

onde na última igualdade usamos os fatos $u_{\mathbf{M}}^{\mathrm{P}}=0$ (pois $\mathbf{M}$ é um P-martingal) e $u_{\mathbf{X}}^{\mathrm{P}}-\mathbf{A}=u_{\mathbf{X}}^{\mathrm{P}}-\mathbf{A}$. Dessa forma, isolando $d \mathbf{A}$ e usando novamente a proposição 4.50 , item ii), temos que*

$$
d \mathbf{A}=d u_{\mathbf{X}}^{\mathrm{P}}+\mathbf{M}^{-1} d \llbracket \mathbf{X}, \mathbf{M} \rrbracket=d u_{\mathbf{X}}^{\mathrm{P}}+d \llbracket \mathbf{X}, \log (\mathbf{M}) \rrbracket,
$$

ou seja,

$$
A_{t}=u_{\mathbf{X}}^{\mathrm{P}}(t)+\llbracket \mathbf{X}, \log (\mathbf{M}) \rrbracket_{t} .
$$

* Note que é nesse ponto que usamos a continuidade de $\mathbf{M}$. 
Assim, temos que (E.5) é equivalente a (E.6) e como $A_{t}$ é contínuo, de variação finita e nulo em $t=0$, provamos o resultado.

\section{E.2.2 A exponencial de Doléans}

Definição E.7. Seja L um martingal local contínuo tal que $L_{0}=0$. Definimos a exponencial de Doléans $\mathcal{E}(\mathbf{L})$ como

$$
\mathcal{E}_{t}(\mathbf{L})=e^{L_{t}-\frac{1}{2} \llbracket \mathbf{L} \rrbracket_{t}}
$$

Note que $\mathbf{L}-\frac{1}{2} \llbracket \mathbf{L} \rrbracket$ é (por definição) um semi-martingal contínuo e como a função exponencial é suficientmente suave, o lema de Itôم ${ }^{*}$ garante que $\mathcal{E}(\mathbf{L})$ é um semi-martingal contínuo. Os próximos resultados justificarão o uso da nomenclatura "exponencial".

Lema E.8. O processo $\mathbf{Z}=\mathcal{E}(\mathbf{L})$ satisfaz $Z_{t}=1+\int_{0}^{t} Z_{s} d L_{s}$ e $Z_{0}=1$. Assim, $\mathbf{Z}$ é martingal local não negativo e portanto um supermartingal.

Demonstração. Seja $X_{t} \doteq L_{t}-\frac{1}{2} \llbracket \mathbf{L} \rrbracket_{t}$. Temos que $\mathbf{X}$ é um semi-martingal contínuo. Pela definição de variação quadrática ${ }^{\dagger}$ segue que $\llbracket \mathbf{X} \rrbracket=\llbracket \mathbf{L} \rrbracket$. Dessa forma $\mathbf{X}=e^{\mathbf{X}}$ e pela fórmula de Itô resulta

$$
d Z_{s}=e^{X_{s}} d X_{s}+\frac{1}{2} e^{X_{s}} d \llbracket \mathbf{X} \rrbracket_{s}=Z_{s} d\left(L_{s}-\frac{1}{2} \llbracket \mathbf{L} \rrbracket_{s}\right)+\frac{1}{2} Z_{s} d \llbracket \mathbf{L} \rrbracket_{s}=Z_{s} d L_{s} .
$$

Pela definição da notação diferencial, temos que $Z_{t}=1+\int_{0}^{t} Z_{s} d L_{s}$ e como $X_{0}=0$ temos também que $Z_{0}=1$. Agora, como $1+\int_{0}^{t} Z_{s} d L_{s}$ é um martingal local contínuo e a função exponencial é positiva, segue que $\mathbf{Z}$ é um martingal local não negativo. Pela proposição 2.57 na página 31, segue que $\mathbf{Z}$ é um supermartingal.

Observe que ainda pela proposição 2.57 , segue que $\mathbf{Z}$ é um martingal se, e somente se, $\mathbf{E}\left(Z_{t}\right)=1$ para todo $t \geq 0$.

Proposição E.9. Sejam $\mathbf{L}$ um martingal local contínuo tal que $L_{0}=0, \mathbf{X}$ um semi-martingal contínuo e denote $E_{t}=\mathcal{E}(\mathbf{L})$. Então toda solução da equação $d X_{s}=X_{s} d L_{s}$ tem a forma $X_{t}=X_{0} E_{t}$.

\footnotetext{
* Mais especificamente, o corolário 4.45 na página 111. † Veja a definição 3.27 na página 57.
} 
Demonstração. Defina $U_{t}=\frac{X_{t}}{E_{t}}$. Pelo lema de Itô, temos que U é um semi-martingal. Como $E_{0}=1$, temos que $U_{0}=X_{0}$ e portanto $X_{t}=X_{0} E_{t}$ se, e somente se, $U_{t}=U_{0}$ para todo $t \geq 0$. Mas como U é semi-martingal, pela observação após a definição 4.47 na página $113, U_{t}=U_{0}$ é equivalente a $d U_{t}=0, t \geq 0$. Ou seja, se mostrarmos que $d U_{t}=0$ para todo $t \geq 0$, a proposição estará provada. Para isto, temos da fórmula de integração por partes* que

$$
d U_{t}=X_{t} d E_{t}^{-1}+E_{t}^{-1} d X_{t}+d \llbracket \mathbf{X}, \mathbf{E}^{-1} \rrbracket_{t}
$$

Agora, por hipótese temos que $\mathbf{E}=1+\mathbf{E} \bullet \mathbf{L}$ e $\mathbf{X}=X_{0}+\mathbf{X} \bullet \mathbf{L}$. Dessa forma, observe

$$
\begin{aligned}
\llbracket \mathbf{E} \rrbracket & =\llbracket 1+\mathbf{E} \bullet \mathbf{L}, 1+\mathbf{E} \bullet \mathbf{L} \rrbracket \\
& =\llbracket \mathbf{E} \bullet \mathbf{L}, \mathbf{E} \bullet \mathbf{L} \rrbracket \\
& =\mathbf{E}^{2} \bullet \llbracket \mathbf{L} \rrbracket
\end{aligned}
$$

Aqui, a primeira igualdade segue da definição de covariação quadrática para semi-martingais (como no lema anterior) e a última segue pela proposição 4.49 na página 113 . Como $\llbracket \mathbf{E} \rrbracket_{0}=0$, segue da definição da notação diferencial que

$$
d \llbracket \mathbf{E} \rrbracket_{t}=E_{t}^{2} d \llbracket \mathbf{L} \rrbracket_{t}
$$

Além disso,

$$
\begin{aligned}
d \llbracket \mathbf{X}, \mathbf{E}^{-1} \rrbracket_{t} & =-E_{t}^{2} d \llbracket \mathbf{X}, \mathbf{E} \rrbracket_{t} \\
& =-E_{t}^{2} X_{t} E_{t} d \llbracket \mathbf{L}, \mathbf{L} \rrbracket_{t} \\
& =-X_{t} E_{t}^{-1} d \llbracket \mathbf{L} \rrbracket_{t} .
\end{aligned}
$$

Onde a primeira igualdade segue da proposição 4.50 na página 115, item i). Observe finalmente que pelo lema de Itô e por (E.8), segue que

$$
\begin{aligned}
d E_{t}^{-1} & =-E_{t}^{-2} d E_{t}+E_{t}^{-3} d \llbracket \mathbf{E} \rrbracket_{t} \\
& =-E_{t}^{-1} d L_{t}+E_{t}^{-1} d \llbracket \mathbf{L} \rrbracket_{t} .
\end{aligned}
$$

Substituindo (E.10), $d X_{t}=X_{t} d L_{t}$ e (E.9) em (E.7) resulta que $d U_{t}=0$, como queríamos.

Corolário E.10. Seja $\mathbf{V}$ um martingal local contínuo d-dimensional e $\boldsymbol{\gamma} \in \mathcal{L}(\mathbf{V})$. Então a solução

\footnotetext{
* Veja a proposição 4.40 na página 101.
} 
geral de

$$
d X_{t}=X_{t} \gamma_{t} \cdot d V_{t}
$$

é $X_{t}=X_{0} \mathcal{E}(\gamma \bullet \mathbf{V})$.

Demonstração. De fato, note que $\gamma_{t} \cdot d V_{t}=d(\gamma \bullet \mathbf{V})_{t}$ e use o resultado anterior com $\mathbf{L}=\gamma \bullet \mathbf{V}$.

\section{E.3 Teorema de Girsanov}

Em finanças é muito comum a seguinte situação: Temos um processo $\mathbf{r}$ que satisfaz a seguinte equação diferencial estocástica

$$
d r_{t}=\alpha_{t} d t+\beta_{t} d W_{t}
$$

onde $\mathbf{W}$ é um movimento Browniano definido em um espaço $\left(\Omega, \mathscr{F},\left\{\mathscr{F}_{t}\right\}, \mathrm{P}\right)$. Em finanças, com objetivo de apreçar derivativos financeiros, é vantajoso* trocar a medida de probabilidade P por uma localmente equivalente Q. Os resultados desta seção nos ajudam a entender como será a dinâmica deste processo nessa nova medida.

Teorema E.11 (Teorema de Girsanov I). Sejam W um movimento Browniano d-dimensional definido em $\left(\Omega, \mathscr{F},\left\{\mathscr{F}_{t}\right\}, \mathrm{P}\right), \mathrm{Q}$ uma medida localmente equivalente à $\mathrm{P}$ e suponha que o processo densidade $M_{t}=\frac{d\left(\mathrm{Q} \mid \mathscr{F}_{t}\right)}{d\left(\mathrm{P} \mid \mathscr{F}_{t}\right)}$ satisfaça $\mathbf{M}=\mathcal{E}(\boldsymbol{\gamma} \bullet \mathbf{W})$, isto é,

$$
\frac{d M_{t}}{M_{t}}=\gamma_{t} d W_{t}, \quad t \geq 0
$$

para algum $\gamma \in \mathcal{L}(\mathbf{W})$. Então o processo $\mathbf{W}^{\mathrm{Q}}$ definido por

$$
W_{t}^{\mathrm{Q}}=W_{t}-\int_{0}^{t} \gamma_{s} d s
$$

é um movimento Browniano em $\left(\Omega, \mathscr{F},\left\{\mathscr{F}_{t}\right\}, \mathrm{Q}\right)$.

Demonstração. De fato, como W é um P-semi-martingal, pela proposição E.6, segue que W é

\footnotetext{
* Consulte [11], por exemplo, para uma discussão aprofundada sobre este assunto.
} 
também um Q-semi-martingal com compensador $u_{\mathbf{W}}^{\mathrm{Q}}$. Defina

$$
\mathbf{W}^{\mathrm{Q}} \doteq \mathbf{W}-u_{\mathbf{W}}^{\mathrm{Q}}
$$

Temos que $\mathbf{W}^{\mathrm{Q}}$ é um martingal local e como $u_{\mathbf{W}}^{\mathrm{Q}}$ é um processo de variação finita, pela definição de covariação quadrática para semi-martingais* temos que

$$
\llbracket\left(\mathbf{W}^{i}\right)^{\mathrm{Q}} \rrbracket_{t}=\llbracket \mathbf{W}^{i} \rrbracket_{t}=t \mathrm{e} \llbracket\left(\mathbf{W}^{i}\right)^{\mathrm{Q}},\left(\mathbf{W}^{j}\right)^{\mathrm{Q}} \rrbracket_{t}=\llbracket \mathbf{W}^{i}, \mathbf{W}^{j} \rrbracket_{t}=0
$$

para $i, j \in\{1,2, \ldots, d\}$ com $i \neq j$. Pela caracterização de Levy (teorema 4.52 na página 118), segue que $\mathbf{W}^{\mathrm{Q}}$ é um movimento Browniano com respeito à medida Q. Falta mostrar que $u_{\mathbf{W}}^{\mathrm{Q}}=\int_{0}^{t} \gamma_{s} d s$. Para isso, usando novamente a proposição E.6, temos que $u_{\mathbf{W}}^{\mathrm{Q}}=u_{\mathbf{W}}^{\mathrm{P}}+\llbracket \mathbf{W}, \log (\mathbf{M}) \rrbracket$. Porém $\mathbf{W}$ é um P-martingal e assim $u_{\mathbf{W}}^{\mathrm{P}}=0$. Ficamos com

$$
u_{\mathbf{W}}^{\mathrm{Q}}=\llbracket \mathbf{W}, \log (\mathbf{M}) \rrbracket
$$

Conforme vimos na seção 4.5 na página 105 sobre a notação vetorial, temos

$$
\llbracket \mathbf{W}, \log (\mathbf{M}) \rrbracket=\left(\llbracket \mathbf{W}^{1}, \log (\mathbf{M}) \rrbracket, \llbracket \mathbf{W}^{2}, \log (\mathbf{M}) \rrbracket, \ldots, \llbracket \mathbf{W}^{d}, \log (\mathbf{M}) \rrbracket\right)^{\prime} .
$$

Agora, como $\mathbf{M}=\mathcal{E}(\boldsymbol{\gamma} \bullet \mathbf{W})=e^{(\boldsymbol{\gamma} \bullet \mathbf{W})-\frac{1}{2} \llbracket \boldsymbol{\gamma} \bullet \mathbf{W} \rrbracket}$, temos que $\log (\mathbf{M})=(\boldsymbol{\gamma} \bullet \mathbf{W})-\frac{1}{2} \llbracket \gamma \bullet \mathbf{W} \rrbracket$. Como $\frac{1}{2} \llbracket \gamma \bullet \mathbf{W} \rrbracket$ é de variação finita, denotando $\gamma^{i}$ a $i$-ésima componente do processo $d$-dimensional $\gamma$, temos pela definição de covariação quadrática para semi-martingais que

$$
\llbracket \mathbf{W}^{j}, \log (\mathbf{M}) \rrbracket=\llbracket \mathbf{W}^{j}, \sum_{\mathbf{i}=\mathbf{1}}^{\mathbf{d}} \boldsymbol{\gamma}^{i} \bullet \mathbf{W}^{i} \rrbracket
$$

Pela bilinearidade da covariação quadrática, propriedades da integral estocástica e da covariação para

* Veja a definição 3.27 na página 57. 
o movimento Browniano, segue que

$$
\begin{aligned}
\llbracket \mathbf{W}^{j}, \sum_{\mathbf{i}=\mathbf{1}}^{\mathbf{d}} \boldsymbol{\gamma}^{\mathbf{i}} \bullet \mathbf{W}^{\mathbf{i}} \rrbracket & =\sum_{i=1}^{d} \gamma^{i} \bullet \llbracket \mathbf{W}^{j}, \mathbf{W}^{i} \rrbracket \\
& =\int_{0}^{j} \gamma_{s}^{j} d s \\
& =\left(\int_{0} \gamma_{s} d s\right)^{j} .
\end{aligned}
$$

Disso resulta

$$
\llbracket \mathbf{W}, \log (\mathbf{M}) \rrbracket_{t}=\int_{0}^{t} \gamma_{s} d s,
$$

como queríamos.

Voltando a discussão do início desta subseção, pelo teorema de Girsanov se encontrarmos um processo $\gamma$ satisfazendo $\frac{d M_{t}}{M_{t}}=\gamma_{t} d W_{t}, \quad t \geq 0$, temos que o movimento Browniano sob a medida $\mathrm{Q}$ satisfaz $W_{t}^{\mathrm{Q}}=W_{t}-\int_{0}^{t} \gamma_{s} d s$, ou em notação diferencial $d W_{t}^{\mathrm{Q}}=d W_{t}-\gamma_{t} d t$ e portanto o processo $\mathbf{r}$ satisfazendo (E.11) tem dinâmica sob a nova medida de probabilidade Q dada por

$$
d r_{t}=\left[\alpha_{t}+\beta_{t} \gamma_{t}\right] d t+\beta_{t} d W_{t}^{\mathrm{Q}}
$$

Em finanças é comum a situação de inicialmente considerar um processo satisfazendo (E.11) e querer eliminar o termo $\alpha_{t} d t$, o chamado termo de drift. Para isso, procuramos um processo $\gamma \in \mathcal{L}(\mathbf{W})$ de tal forma que $\alpha_{t}+\beta_{t} \gamma_{t}=0$ e a questão é se existe alguma medida de probabilidade $\mathrm{Q}$, localmente equivalente à $\mathrm{P}$ tal que $W_{t}^{\mathrm{Q}}=W_{t}-\int_{0}^{t} \gamma_{s} d s$ seja um movimento Browniano com respeito à Q. Nesse caso a dinâmica de $\mathbf{r}$ sob a medida $\mathrm{Q}$ seria dada por $d r_{t}=\beta_{t} d W_{t}^{\mathrm{Q}}$. É esse tipo de recíproca do teorema de Girsanov que os próximos resultados tratam.

Proposição E.12. Sejam $T>0$, W um movimento Browniano definido com respeito ao espaço filtrado $\left(\Omega, \mathscr{F},\left\{\mathscr{F}_{t}\right\}, \mathrm{P}\right)$ em $[0, T]$ e $\boldsymbol{\gamma} \in \mathcal{L}(\mathbf{W})$ tal que $M_{t}=\mathcal{E}_{t}(\boldsymbol{\gamma} \bullet \mathbf{W})$ seja um P-martingal em $[0, T]$. Então existe uma medida $\mathrm{Q}$, localmente equivalente à $\mathrm{P}$ com respeito a $\left\{\mathscr{F}_{t}\right\}_{t \in[0, T]}$ tal que o 
processo

$$
\widetilde{W}_{t}=W_{t}-\int_{0}^{t} \gamma(s) d s, \quad t \in[0, T]
$$

é um movimento Browniano com respeito ao espaço filtrado $\left(\Omega, \mathscr{F},\left\{\mathscr{F}_{t}\right\}, \mathrm{Q}\right)$ em $[0, T]$.

Demonstração. Defina a medida $\mathrm{Q}_{T}=\mathrm{Q}: \mathscr{F}_{T} \rightarrow \mathbb{R}_{+}$por

$$
\mathrm{Q}(A)=\int_{A} M_{T} d \mathrm{P}, \quad \forall A \in \mathscr{F}_{T},
$$

equivalentemente, $\frac{d \mathrm{Q}}{d\left(\mathrm{P} \mid \mathscr{F}_{t}\right)}=M_{T}$. Como $\left\{M_{t}\right\}_{t \in[0, T]}$ é um martingal, pela observação após a demonstração do lema E.8, temos que $\mathbf{E}\left(M_{T}\right)=1$. Isso implica que a nossa medida $Q$ é de fato uma medida de probabilidade. Além disso, como por hipótese, $\left\{M_{t}\right\}_{t \in[0, T]}$ é estritamente positivo, o teorema de Radon-Nikodym garante que $\mathrm{Q}$ e $\mathrm{P}_{T}$ são equivalentes e portanto são localmente equivalentes com respeito a $\left\{\mathscr{F}_{t}\right\}_{t \in[0, T]}$. Pelo teorema E.3 segue que o processo densidade $\frac{d\left(\mathrm{Q} \mid \mathscr{F}_{t}\right)}{d\left(\mathrm{P} \mid \mathscr{F}_{t}\right)}$ é um P-martingal.

Agora, note que para terminar a demonstração, basta mostrar que os processos $\left\{M_{t}\right\}$ e $\left\{\frac{d\left(\mathrm{Q} \mid \mathscr{F}_{t}\right)}{d\left(\mathrm{P} \mid \mathscr{F}_{t}\right)}\right\}$ são iguais. Dessa forma estamos sob as condições do teorema de Girsanov e portanto vale (E.12). Para isso, note inicialmente que por construção $M_{T}=\frac{d\left(\mathrm{Q} \mid \mathscr{F}_{T}\right)}{d\left(\mathrm{P} \mid \mathscr{F}_{T}\right)}$. Como $\left\{M_{t}\right\}$ e $\left\{\frac{d\left(\mathrm{Q} \mid \mathscr{F}_{t}\right)}{d\left(\mathrm{P} \mid \mathscr{F}_{t}\right)}\right\}$ são martingais, a diferença entre esses dois processos também o é, assim, para $t \in[0, T]$,

$$
0=\mathbf{E}\left(M_{T}-\frac{d\left(\mathrm{Q} \mid \mathscr{F}_{T}\right)}{d\left(\mathrm{P} \mid \mathscr{F}_{T}\right)} \| \mathscr{F}_{t}\right)=M_{t}-\frac{d\left(\mathrm{Q} \mid \mathscr{F}_{t}\right)}{d\left(\mathrm{P} \mid \mathscr{F}_{t}\right)}
$$

ou seja, $M_{t}=\frac{d\left(\mathrm{Q} \mid \mathscr{F}_{t}\right)}{d\left(\mathrm{P} \mid \mathscr{F}_{t}\right)}$, q. s. para todo $t \in[0, T]$. A continuidade dos processos implica que $\left\{M_{t}\right\}$ e $\left\{\frac{d\left(\mathrm{Q} \mid \mathscr{F}_{t}\right)}{d\left(\mathrm{P} \mid \mathscr{F}_{t}\right)}\right\}$ são indistinguíveis, como queríamos.

O leitor atento aos detalhes percebeu que no resultado anterior a medida encontrada Q é equivalente a $\mathrm{P}$ na $\sigma$-álgebra $\mathscr{F}_{T}$. Desta forma o movimento Browniano em $\mathrm{Q}$ esta definido somente no intervalo finito $[0, T]$. O problema é mais delicado se dado $\gamma \in \mathcal{L}(\mathbf{W})$, tentarmos encontrar uma medida $\mathrm{Q}$ definida em $\mathscr{F}_{\infty}$ que é localmente equivalente à P. Para ilustrar este ponto, considere $\mathbf{W}$ um movimento Browniano unidimensional definido em $[0, \infty)$ e $\mu$ um número real positivo. Defina $\gamma(t) \equiv \mu$. Afirmamos que não existe uma medida de probabilidade Q absolutamente contínua com respeito à $\mathrm{P}$ em $\mathscr{F}_{\infty}$ tal que $\widetilde{W}_{t}=W_{t}-\int_{0}^{t} \mu d s=W_{t}-\mu t$ seja um movimento Browniano sob Q. De 
fato suponha por absurdo que tal medida existisse. Defina

$$
A \doteq\left\{\lim _{t \rightarrow \infty} \frac{\widetilde{W}_{t}}{t}=0\right\}=\left\{\lim _{t \rightarrow \infty} \frac{W_{t}}{t}=\mu\right\} .
$$

Como W é P-movimento Browniano temos* que $\mathrm{P}(A)=0$ e $\mathrm{Q}(A)=0$. Ou seja, Q não é absolutamente contínua com respeito à $\mathrm{P}$ em $\mathscr{F}_{\infty}$. O que ocorre é que não é possivel construir uma medida localmente equivalente a partir de um martingal quando estamos trabalhando com a filtração completa e aumentada gerada por um movimento Browniano em $[0, \infty)$.

No entanto, quando filtração não for completa, é possivel generalizar a proposição anterior para $[0, \infty)$, mas para isto precisamos estar trabalhando na configuração canônica. Vamos rapidamente falar um pouco sobre isso. Seja $\mathcal{C}=\mathcal{C}\left(\mathbb{R}_{+}, \mathbb{R}\right)$ o conjunto de todas as funções contínuas $\omega:[0, \infty) \rightarrow \mathbb{R}$ munido com a métrica da convergência uniforme, isto é, para $\omega, \widetilde{\omega} \in \mathcal{C}$

$$
\rho(\omega, \widetilde{\omega})=\sum_{j=1}^{\infty}\left(\frac{1}{2}\right)^{j} \frac{\rho_{j}(\omega, \widetilde{\omega})}{1+\rho_{j}(\omega, \widetilde{\omega})}
$$

onde

$$
\rho_{j}(\omega, \widetilde{\omega})=\sup _{0 \leq t \leq j}|\omega(t)-\widetilde{\omega}(t)| .
$$

Seja $\mathscr{B}(\mathcal{C})$ a $\sigma$-àlgebra de Borel induzida pela métrica $\rho$ e defina a projeção $\pi_{t}: \mathcal{C} \rightarrow \mathbb{R}$ por $\pi_{t}(\omega)=\omega_{t}$. Pode-se provar que a família $\left\{\mathscr{C}_{t}\right\}$, com $\mathscr{C}_{t} \doteq \sigma\left(\pi_{s}: s \leq t\right)$ define uma filtração em $\mathcal{C}$. Denotare$\operatorname{mos}\left(\mathcal{C}^{d},\left\{\mathscr{C}_{t}^{d}\right\}, \mathscr{C}^{d}\right)$ a versão $d$-dimensional deste espaço. Agora suponha que $\mathbf{W}$ é um movimento Browniano $d$-dimensional com trajétorias q. s. contínuas definido no espaço completo $(\Omega,\{\mathscr{F} t\}, \mathscr{F}, \mathrm{P})$. Então, W induz uma medida de probabilidade (chamada medida de Wiener) $\widetilde{\mathrm{P}}$ em $\left(\mathcal{C}^{d},\left\{\mathscr{C}_{t}^{d}\right\}, \mathscr{C}^{d}\right)$ definida por

$$
\widetilde{\mathrm{P}}(A)=\mathrm{P}(X . \in A),
$$

para todo $A \in \mathscr{C}^{d}$. Dessa maneira, processo estocástico $\widetilde{\mathbf{W}}$ definido no espaço $\left(\mathcal{C}^{d},\left\{\mathscr{C}_{t}^{d}\right\}, \mathscr{C}^{d}, \widetilde{\mathrm{P}}\right)$ pela relação

$$
\widetilde{W}(t, \omega) \doteq \omega_{t}
$$

tem a mesma lei de $\mathbf{X}$. Quando consideramos o espaço $\left(\mathcal{C}^{d},\left\{\mathscr{C}_{t}^{d}\right\}, \mathscr{C}^{d}, \widetilde{\mathrm{P}}\right)$ completado, dizemos que ele é configuração canônica para $\mathbf{W}$.

\footnotetext{
* Para uma demonstração deste fato, veja por exemplo [9], página 24.
} 
O motivo de introduzir a configuração canônica é que se não estivermos trabalhando com filtração completa pode ser impossível definir a integral estocástica, exceto, se estivermos trabalhando sob a configuração canônica e nessa situação é possivel generalizar a proposição anterior para $[0, \infty)$. Enunciaremos sem demonstração essa generalização. Para mais detalhes sobre esses assuntos veja [23], [9] $\mathrm{e}[15]$.

Proposição E.13 (Teorema de Girsanov II). Sejam W um movimento Browniano definido sob a configuração canônica no espaço $\left(\Omega, \mathscr{F},\left\{\mathscr{F}_{t}\right\}, \mathrm{P}\right)$ e $\gamma \in \mathcal{L}(\mathbf{W})$ tal que $M_{t}=\mathcal{E}_{t}(\boldsymbol{\gamma} \bullet \mathbf{W})$ seja um martingal. Para cada $t \geq 0$ defina a medida $\mathrm{Q}_{t}$ em $\mathscr{F}_{t}$ por

$$
\frac{d \mathrm{Q}_{t}}{d\left(\mathrm{P} \mid \mathscr{F}_{t}\right)}=M_{t}
$$

ou, equivalentemente,

$$
Q_{t}(A)=\mathbf{E}_{\mathrm{P}}\left(M_{t} \mathbb{1}_{[A]}\right), \quad A \in \mathscr{F}_{t} .
$$

Então existe uma única medida de probabilidade $\mathrm{Q}$ definida em $\mathscr{F}_{\infty}$ tal que $\mathrm{Q} \mid \mathscr{F}_{t}=\mathrm{Q}_{t}$ e portanto $\frac{d\left(\mathrm{Q} \mid \mathscr{F}_{t}\right)}{d\left(\mathrm{P} \mid \mathscr{F}_{t}\right)}=M_{t}$, para todo $t \geq 0$ e com essa medida, o processo $\widetilde{W}_{t}=W_{t}-\int_{0}^{t} \gamma_{s} d s, t \geq 0$ é um Q-movimento Browniano.

\section{E.3.1 A condição de Novikov}

Percebe-se que para a aplicação do teorema de Girsanov é importante saber quando $M_{t}=\mathcal{E}_{t}(\boldsymbol{\gamma} \bullet \mathbf{W})$ é um martingal. O próximo teorema dá uma condição suficiente para isso. Uma demonstração pode ser encontrada em [23].

Teorema E.14 (Condição de Novikov). Seja $\mathbf{L}$ um martingal local contínuo com $L_{0}=0$, adaptado à uma filtração aumentada gerada por um movimento Browniano e suponha que

$$
\mathbf{E}_{\mathrm{P}}\left(e^{\frac{1}{2} \llbracket \mathbf{L} \rrbracket_{t}}\right)<\infty, \quad \forall 0 \leq t<\infty
$$

Então $\mathcal{E}_{t}(\mathbf{L})=e^{L_{t}-\frac{1}{2} \llbracket \mathbf{L} \rrbracket_{t}}$ é um martingal.

Tendo em vista a aplicação do teorema de Girsanov, temos como corolário a seguinte versão da condição de Novikov quando $\mathbf{L}=\gamma \bullet \mathbf{W}$.

Corolário E.15. Seja $\mathbf{W}$ um movimento Browniano d-dimensional e $\boldsymbol{\gamma} \in \mathcal{L}(\mathbf{W})$. Então o super- 
martingal $\mathcal{E}(\boldsymbol{\gamma} \bullet \mathbf{W})$ é um martingal quando a seguinte condição estiver satisfeita:

$$
\mathbf{E}_{\mathbf{P}}\left(e^{\left(\frac{1}{2} \int_{0}^{t}\left\|\gamma_{s}\right\|^{2} d s\right)}\right)<\infty, \quad \forall t \geq 0
$$

Demonstração. Inicialmente, lembre-se da notação vetorial vista na seção 4.5 na página 105. Temos que $\gamma \bullet \mathbf{W}=\sum_{j} \gamma^{j} \bullet \mathbf{W}^{j}$. Agora, pela bilinearidade da covariação quadrática e como $\llbracket \mathbf{W}^{i}, \mathbf{W}^{j} \rrbracket_{t}=0$, se $i \neq j$ e $\llbracket \mathbf{W}^{i}, \mathbf{W}^{j} \rrbracket_{t}=t$, se $i=j$, temos que

$$
\begin{aligned}
\llbracket \boldsymbol{\gamma} \bullet \mathbf{W} \rrbracket & =\llbracket \boldsymbol{\gamma} \bullet \mathbf{W}, \boldsymbol{\gamma} \bullet \mathbf{W} \rrbracket \\
& =\sum_{i, j=1}^{d} \gamma^{i} \boldsymbol{\gamma}^{j} \bullet \llbracket \mathbf{W}^{i}, \mathbf{W}^{j} \rrbracket \\
& =\sum_{j=1}^{d} \int_{0}\left(\gamma^{j}(s)\right)^{2} d s \\
& =\int_{0}\|\gamma(s)\|^{2} d s .
\end{aligned}
$$

Dessa forma, pela condição de Novikov, $\mathcal{E}(\boldsymbol{\gamma} \bullet \mathbf{W})$ é um martingal quando

$$
\mathbf{E}_{\mathrm{P}}\left(e^{\frac{1}{2} \llbracket \gamma \bullet \mathbf{W} \rrbracket_{t}}\right)=\mathbf{E}_{\mathrm{P}}\left(e^{\frac{1}{2} \int_{0}^{t}\|\gamma(s)\|^{2} d s}\right)<\infty
$$

para todo $t \geq 0$. Como queríamos.

Observamos que quando estivermos trabalhando em um intervalo de tempo finito $[0, T], \mathcal{E}(\boldsymbol{\gamma} \bullet \mathbf{W})$ é um martingal em $[0, T]$ quando

$$
\mathbf{E}_{\mathrm{P}}\left(e^{\frac{1}{2} \int_{0}^{T}\|\gamma(s)\|^{2} d s}\right)<\infty
$$

A demonstração desse fato segue observando que a prova para a condição de Novikov para o intervalo de tempo finito $[0, T]$ é essencialmente a mesma para o caso infinito. 


\section{Referências Bibliográficas}

[1] P. Billingsley, Probability and measure, third ed., Wiley-Interscience, 1995. 154, 158, 159, 162, $171,174,178$

[2] Adam Bobrowski, Functional analysis for probability and stochastic processes, Cambridge University Press, 2005. 180

[3] Mark H.A. Davis, Construction of brownian motion, Disponível na internet, 2004.

[4] Robert B. Ash \& Catherine A. Doleans-Dade, Probability $\&$ measure theory, second ed., Academic Press. 166

[5] D. Duffie, Dynamic asset pricing theory, third ed., Princeton University Press, 2001.

[6] R. Durret, Stochastic calculus - a practical introduction, CRC Press, 1996. 38

[7] A. Etheridge, A course in financial calculus, Cambridge University Press, 2002. 61

[8] G.B. Folland, Real analysis: Modern techniques and their applications, second ed., WileyInterscience, 1999. 62

[9] P. J. Hunt \& J. E. Kennedy, Financial derivatives in theory and practice, first ed., Wiley, 2004. 137, 192, 193

[10] Davar Khoshnevisan, Notes on probability theory, Disponível na internet, 2003.

[11] N.H. Bingham \& R. Kiesel, Risk-neutral valuation: Pricing and hedging of financial derivatives, second ed., Springer, 2004. 188

[12] R. Korn \& E. Korn, Option pricing and portfolio optimization, AMS, 2001.

[13] Hui-Hsiung Kuo, Introduction to stochastic integration, Springer, 2005. 128

[14] K. Athreya \& S. Lahiri, Measure theory and probability theory, Springer, 2006. 19

[15] P. Medvegyev, Stochastic integration theory, Oxford University Press, 2007. 3, 4, 19, 193 
[16] M. Meyer, Continuous stochastic calculus with application to finance, CRC Press, 2000. 4, 38, 151,152

[17] M. Métivier, Semimartingales: A course on stochastic processes, Walter de Gruyter, 1980. 3

[18] S. N. Neftci, Introduction to the mathematics of financial derivatives, second ed., Academic Press, 2000. 150

[19] Lars T. Nielsen, Pricing and hedging of financial derivatives, Oxford University Press, 1999. 165

[20] B. Oksendal, Stochastic differential equations: An introduction with applications, sixth ed., Springer, 2006. 150

[21] P. E. Protter, Stochastic integration and differential equations, second ed., Springer-Verlag, 2004. 3

[22] R. Jarrow \& P. E. Protter, A short history of stochastic integration and mathematical finance: the early years, 1880-1970, IMS Lecture Notes Monogr 45 (2004), 75-91. 4

[23] I. Karatzas \& S. Shreve, Brownian motion and stochastic calculus, second ed., Springer, 1991. $3,4,20,127,130,138,140,184,193$

[24] S. Shreve, Stochastic calculus for finance ii: Continuous-time models, Springer, 2008.

[25] L. Koralov \& Y. G. Sinai, Theory of probability and random processes, second ed., Springer, 2007.

[26] D. Williams, Probability with martingales, Cambridge University Press, 1991.

[27] J. Yeh, Martingales and stochastic analysis, World Scientific, 2005. 12, 61, 155, 156

[28] _ Real analysis: Theory of measure and integration, World Scientific, 2006. 8, 35, 66, 164

[29] D. Revuz \& M. Yor, Continuous martingale and brownian motion, third ed., Springer, 1999. 3, $4,18,38$ 